$\begin{array}{llllllllllllllllllll}\text { C } & \text { A } & \text { N } & \text { A } & \text { D } & \text { I } & \text { A } & \text { N } & & \text { J } & \text { O } & \text { U } & \text { R } & \text { N } & \text { A } & \text { L } & & \text { O } & \text { F }\end{array}$

INFECTIOUS DISEASES \& MEDICAL MICROBIOLOGY

JOURNAL CANADIEN DES MALADIES INFECTIEUSES ET DE LA MICROBIOLOGIE MEDICALE

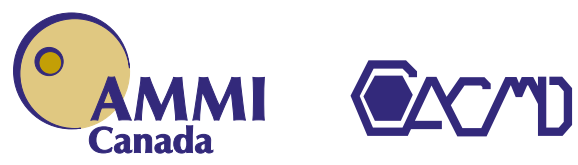

\title{
AMMI Canada - CACMID Annual Conference
}

April 16-18, 2015 • Charlottetown, Prince Edward Island

\section{ORAL PRESENTATIONS}

Thursday, April 16, 2015

\begin{tabular}{|c|c|}
\hline Session A & A01-A04 \\
\hline Session B & B01-B04 \\
\hline Session C & C01-C04 \\
\hline Session D & D01-D04 \\
\hline
\end{tabular}

Friday, April 17, 2015

\begin{tabular}{|lc|}
\hline Session E & E01-E06 \\
\hline Session F & F01-F06 \\
\hline Session G & G01-G06 \\
\hline Session H & H01-H06 \\
\hline
\end{tabular}

Saturday, April 18, 2015

\begin{tabular}{|lr|}
\hline Session I & I01-I05 \\
\hline Session J & J01-J05 \\
\hline Session K & K01-K05 \\
\hline Session L & L01-L05 \\
\hline
\end{tabular}

\section{POSTER PRESENTATIONS}

Thursday, April 16 and Friday, April 17, 2015

P01-P31

\section{STUDENT POSTER PRESENTATIONS}

Thursday, April 16 and Friday April 17, 2015

SP01-SP49

\section{INNOVATION ACADEMY POSTER PRESENTATIONS}

Thursday, April 16 and Friday April 17, 2015

IA01-IA03

\section{CLINICAL VIGNETTE POSTER PRESENTATIONS}

Thursday, April 16 and Friday April 17, 2015

CV01-CV04

\section{AUTHOR INDEX}




\section{ORAL PRESENTATIONS}

\section{4:45-15:45 Oral Presentations: Session A Room: Henry, Delta Prince Edward}

\section{A01}

\section{SUSCEPTIBILITY RATES AND SPECIES IDENTIFICATION OF CAMPYLOBACTER STRAINS ISOLATED FROM HUMAN ENTERIC INFECTIONS IN A NEW BRUNSWICK REGIONAL HOSPITAL}

R Garceau ${ }^{1}$, R Raafat Gad ${ }^{2}$, M Mallet ${ }^{3}$

${ }^{1}$ Centre hospitalier universitaire Dr G L Dumont, Moncton; ${ }^{2}$ New Brunswick Public Health, Fredericton; ${ }^{3}$ Pêches et Océans Canada, Moncton, NB

OBJECTIVES: Campylobacter (C) is a common human enteropathogen. Published data on susceptibility rates from human Canadian Campylobacter isolates is scarce. We analyzed the characteristics of a 38 month cohort of Campylobacter strains isolated from stool specimens in a New Brunswick regional laboratory. We also present the susceptibility data of a subset of $\mathrm{C}$ jejuni isolates to ciprofloxacin (cip) and erythromycin (ery).

METHODS: We retrieved data from stool cultures collected between August of 2011 and October of 2014 in which Campylobacter was isolated. We used Campylobacter CNA agar as the selective medium. Identification was performed using published methods. Isolates not identified as $\mathrm{C}$ jejuni sp jejuni or $\mathrm{C}$ coli were identified by sequencing of the cpn60 gene at a reference laboratory. Susceptibility of $\mathrm{C}$ jejuni to cip and ery was determined by Kirby-Bauer (KB) since May of 2013, using criteria described by Gaudreau. Travel history was obtained from New Brunswick public health.

RESULTS: Campylobacter was isolated in $2.1 \%$ of samples. 82 separate episodes of enteritis were identified. Campylobacter was grown from all samples collected in $85 \%$ of enteritis cases. We isolated: C jejuni (72\%), C lari (10\%), C coli (7\%), C upsaliensis (5\%), $2 \%$ of other species and $4 \%$ of isolates not further identified. 29 isolates of $\mathrm{C}$ jejuni were tested by KB. Resistance to nalidixic acid (na) was seen in 6/7 of cip resistant but in no cip susceptible strains. 76\% (22/29) of C jejuni isolates were susceptible to cip and 97\% (28/29) to ery. Acquisition outside Canada was seen in 5/7 of isolates resistant to cip, but in only 1/22 of cip susceptible ones. Based on a Bayesian analysis with binomial distribution, Beta(1,1) prior and 10,000 simulations, there was a $99.99 \%$ probability that the percentage of resistant cases to cip was higher for contamination outside Canada.

CONCLUSIONS: $\mathrm{C}$ jejuni and $\mathrm{C}$ lari are the most common species isolated in our laboratory. Most cases of $\mathrm{C}$ jejuni resistant to cip in our area are acquired outside Canada. Macrolides such as azithromycin are the therapeutic agents of choice in New Brunswick, especially if the infection is acquired outside Canada.

\section{A02 \\ IDENTIFICATION OF SHIGA-TOXIN PRODUCING ESCHERICHIA COLI (STEC) FROM HUMAN STOOL SPECIMENS USING A TWO-STEP ALGORITHM: CHROMOGENIC MEDIA (CM) AND ENZYME IMMUNOASSAY (EIA)}

C Barth ${ }^{1}$, N Neudorf ${ }^{2}$, D Daku², A Obarianyk ${ }^{2}$, R McDonald ${ }^{2}$, PN Levett ${ }^{2}$, GB Horsman ${ }^{2}$, N Mina ${ }^{2}$, J Minion' ${ }^{1}$, K Malejczyk $^{1}$ ${ }^{1}$ Regina General Hospital Medical Laboratory; ${ }^{2}$ Saskatchewan Disease Control Laboratory, Regina, SK

BACKGROUND: Shiga toxin-producing Escherichia coli (STEC) are responsible for haemorrhagic colitis and potentially more serious complications including, hemolytic uremic syndrome (HUS) and death .The most common serotype of STEC is O157:H7, however as many as $30-50 \%$ of STEC infections are caused by non-O157 E. coli. We evaluated a two-step protocol using the STEC CM and Shiga-toxin EIA to screen for O157 and non-O157 STEC.
METHODS: Clinical stool specimens received at the Regina General Hospital medical microbiology laboratory between June $1^{\text {st }}$ and October $31^{\text {st }}$, 2014 were prospectively evaluated for presence of STEC by being plated onto routine media as per established protocol (BAP, MAC, XLD, CAMPY and E. coli $\mathrm{O} 157 \mathrm{CM}$ ) as well as the new STEC CM. Mauve or Brown colonies on the STEC CM identified as E. coli using MALDI-TOF (Bio Merieux, Vitek MS) were then tested for Shiga toxin production using the EIA. All STEC isolates were sent to the Saskatchewan Disease Control Reference Laboratory (SDCL) for confirmation of Shiga-toxin genes using established protocols. Samples positive by PCR were sent to the National Microbiology Laboratory (NML) in Winnipeg for serotyping.

RESULTS: A total of 1589 stool specimens were screened for STEC. Thirtyfour samples were incompletely worked up and therefore were excluded from the subsequent analysis. 1026 (66.0\%) of specimens showed no growth on STEC CM. Clinically insignificant growth (colony colour other than brown or mauve) was detected in 353 (22.7\%) specimens. 166 (10.7\%) specimens had mauve/brown colonies that needed further workup. Of those, $47.6 \%$ identified as an organism other than E. coli (32\% M. morganii, $22.7 \%$ E. hermanii, $13.3 \%$ Proteus sp., $8 \%$ Enterobacter sp.). Total of 87 (5.5\%) specimens had brown/mauve colonies identified as E. coli from the STEC plates and were subsequently tested using the Shiga toxin EIA. 10 specimens $(0.64 \%)$ tested positive for Shiga toxin by EIA and $100 \%$ of them were confirmed to be positive by PCR. 49 E. coli isolated from the STEC plates which were found to be negative by Shiga toxin EIA were sent to SDCL for Shiga-toxin PCR testing and 100\% were confirmed to be negative. CONCLUSION: Two step algorithm using the STEC CM and Shigatoxin EIA in an area of low prevalence is an effective method for the detection of $\mathrm{O} 157$ and common non-O157 STEC.

\section{A03}

\section{DETECTION OF ENTERIC PATHOGENS IN PATIENTS} EXPERIENCING DIARRHEA BY MULTI-ANALYTE PLATFORMS $\underline{L C h u i}^{1,2}, \mathrm{~S}$ Latonas $^{2}$

${ }^{1}$ Provincial Laboratory for Public Health; ${ }^{2}$ University of Alberta, Edmonton, $\mathrm{AB}$

BACKGROUND: Diarrheal disease, a major public health concern and a leading cause of morbidity and mortality worldwide, may be caused by a wide variety of pathogens which makes diagnosis difficult. Recently, multianalyte platform has emerged as a diagnostic modality that enables the detection of multiple diarrhea causing pathogens simultaneously.

OBJECTIVES: To evaluate the utility of the Luminex xTAG GPP in diagnosing diarrheal disease and compare its performance to that of the Seegene Seeplex ${ }^{\circledR}$ Diarrhea-V ACE assay and routine microbiology testing. MATERIALS AND METHODS: A total of 811 clinical stool samples were collected from patients with suspected gastroenteritis. Community samples $(n=473)$ were sent for routine bacterial culture and hospital samples $(n=338)$ were submitted from the University of Alberta Hospital for Clostridium difficile enzyme immunoassay as specified by the ordering physician. All samples were then tested using the Luminex xTAG® GPP and Seeplex ${ }^{\circledR}$ Diarrhea-V ACE assays which detect 15 (9 bacteria, 3 viruses, and 3 parasites) and 14 (10 bacteria and 4 viruses) target pathogens respectively. RESULTS: Both the Luminex and Seeplex ${ }^{\circledR}$ Diarrhea-V ACE assays detected bacterial pathogens in a higher percentage of community samples than did routine bacterial culture, as well as a higher rate of co-infection. Since viral testing is not part of the routine testing algorithm, none of the virus was detected in the routine laboratory. However, different viruses were detected by the molecular tests. For pathogens common to both multi-analyte detection assays, a higher percentage of samples tested positive with the Luminex xTAG ${ }^{\circledR}$ GPP $(30.95 \%)$ than with the Seegene Seeplex® Diarrhea-V ACE assay (25.77\%).

CONCLUSION: These results suggest that both multi-analyte detection assays are superior to routine bacterial testing for diarrheal disease in Alberta. The utilization of either assay will allow the detection of pathogens associated with diarrheal disease. Both assays have pros and cons dependent on the target pathogen. Implementing multi-analyte detection platforms in routine diagnostics would have the potential to improve lab turnaround time and patient care and outcome, including a reduction in inappropriate antibiotic use. 


\section{A04}

PERFORMANCE AND EFFICIENCY OF TWO ALGORITHMS FOR CLOSTRIDIUM DIFFICILE ASSOCIATED DIARRHEA (CDAD) DIAGNOSIS FOR A PROVINCIAL MICROBIOLOGY NETWORK

P Daley ${ }^{1,2}$, K Wells ${ }^{2}$, J Cole ${ }^{2}$, S Tiller ${ }^{2}$, R Needle ${ }^{2}$, M Hughes $^{1}$, C Penney ${ }^{1}$

${ }^{1}$ Memorial University; ${ }^{2}$ Eastern Health Region, St John's, NL BACKGROUND: Algorithms containing both EIA and PCR offer reduced cost and more point of care application as compared to PCR on all stool specimens. EIA for both GDH and Toxin is applied to all specimens and PCR only used for discrepant results. We compared two algorithms to PCR alone.

METHODS: Algorithm 1 combined GDH/Toxin EIA (QuikChek, Alere, $\$ 14,3$ minutes hands-on time) and PCR (Illumigene, Meridian Biosciences, \$24, 7 minutes). Algorithm 2 combined GDH/Toxin EIA and PCR (Amplivue, Quidel, \$20, 5 minutes). Reference method was PCR alone (Illumigene). 200 consecutive liquid stool specimens submitted to a regional microbiology lab between October and December 2014 were included. RESULTS: Mean age was 61.9 years (SD 21.2), 120 (60.0\%) were female and $114(57.0 \%)$ were inpatients (mean length of stay 15.7 days (SD 48.9). In both algorithms, only 33/200 (16.5\%) specimens required PCR. Algorithm 1 had sensitivity of $100 \%$ (95\% CI 100-100\%), specificity of $99 \%$ (98-100\%), and total cost of $\$ 4007.50$. Algorithm 2 had sensitivity of $94 \%$ (92-98\%), specificity of $98 \%$ (96-100\%), and total cost of $\$ 3842.50$. Reference standard cost $\$ 5500.00$.

CONCLUSIONS: Algorithm diagnosis reduced cost without sacrificing performance. Algorithm 1 offered better performance (although reference method was included in algorithm). Algorithm diagnosis could be applied in rural laboratory locations with referral of discrepants to regional centers.

\section{4:45-15:45 Oral Presentations: Session B Room: Johnson, Delta Prince Edward}

\section{B01}

\section{EVALUATION OF MALDI-TOF FOR IDENTIFICATION OF CAMPYLOBACTER, ARCOBACTER, HELICOBACTER, AND RELATED ORGANISMS}

L Moses $^{1}$, M Majcher ${ }^{1}$, K Trout-Yakel ${ }^{1}$, R Ilagan ${ }^{1}$, T Drew ${ }^{1}$, P Chong ${ }^{2}$, S McCorrister $^{2}$, G Westmacott ${ }^{2}, \mathrm{C} \mathrm{Nadon}^{2}$

${ }^{1}$ Enteric Diseases Program; ${ }^{2}$ Proteomics \& Mass Spectrometry Core Facility, National Microbiology Laboratory, Winnipeg, MB

OBJECTIVE: MALDI-TOF MS was evaluated for its effectiveness as an identification tool for Campylobacter and related organisms compared to conventional biochemical and molecular methods.

METHODS: For validation, 399 clinical and reference isolates, representing 39 species, belonging to the genera Campylobacter, Arcobacter, Helicobacter, Wolinella, and Bacteriodes were subjected to conventional short or full biochemical plus molecular testing (PCR-RFLP, species-specific PCR, 16S sequencing), as well as MALDI-TOF analysis in parallel. In a second implementation stage MALDI-TOF analysis was used as the initial identification screen followed by selective confirmatory conventional testing. RESULTS: MALDI-TOF results were in agreement with the conventional results at the species level: $100 \%$ (322/322) for Campylobacter sp., $100 \%$ (22/22) for Arcobacter sp., 84\% (46/55) for Helicobacter sp. 100\% (1/1) for Wolinella sp., and 100\% (1/1) for Bacteriodes sp. However, even for isolates that were not in perfect agreement at the species level, MALDITOF correctly identified the genus for every isolate in this study with the exception of $1 \mathrm{H}$. pullorum and $3 \mathrm{H}$. pylori isolates which generated no reliable information. The conventional biochemical testing allowed for the determination of Campylobacter biotypes, as well as biochemical variants such as hippurate-negative C. jejuni, urea-positive C. lari, and aberrant antimicrobial susceptibility profiles. While MALDI-TOF was not able to discriminate among some biotypes or biochemical variants, it was able to detect a C. fetus isolate belonging to a distinct lineage of a recently reported third subspecies. Conventional identification was completed in
5 to 21 consecutive days (short and full biochemicals, respectively) whereas MALDI-TOF analysis was completed in 2 to 3 days.

CONCLUSIONS: The identification of Campylobacter and related organisms by MALDI-TOF was an efficient and effective means of identification offering a substantial reduction in time and resources. Although it was unable to determine biotypes, biochemical variants, or molecular anomalies, due to its speed and accuracy, identification by MALDI-TOF analysis is recommended for use in a clinical or reference laboratory to either supplement or substitute conventional species-level identification methods for Campylobacter, Arcobacter, Helicobacter, Wolinella, or Bacteriodes.

\section{B02}

COMPARATIVE EVALUATION OF BBL CHROMAGAR ORIENTATION AND CONVENTIONAL CULTURE APPROACH FOR IDENTIFICATION AND SUSCEPTIBILITY OF UROPATHOGENS

N Mina, T Ash, R Kitzul, CY Turenne, PN Levett, GB Horsman Saskatchewan Disease Control Laboratory, Regina, SK

OBJECTIVES: Chromogenic agar offers the potential to identify uropathogens while reducing laboratory workload by decreasing subcultures before being tested on automated identification and susceptibility systems. The purpose of this study is to compare the performance of direct inoculation of colonies from BBL Chromagar Orientation to conventional urine culture methods.

METHODS: One hundred eight urine samples were inoculated simultaneously onto blood agar (BA), MacConkey agar (MAC), and Chromagar and incubated for 18-24 hours aerobically at 35C. Colonies from these media were tested by Microscan (Siemens) for identification and susceptibility. Results from conventional media versus chromogenic agar were compared by calculating the essential and categorical agreement for each antibiotic and the percentage of concordant identification. Prior to validation of this test, cephalothin results were used to predict oral cephalosporin susceptibility for uncomplicated urinary tract infections due to certain Enterobacteriaceae.

RESULTS: Identification results from Microscan were identical for organisms isolated from BA/MAC and Chromagar. Categorical agreement for all antimicrobials tested was greater than $90 \%$ except for cephalothin $(14 \%$ minor errors) and piperacillin (2.6\% very major error). Essential agreement was greater than $90 \%$ except for cephalothin (85\%) and piperacillin (88.3\%). CONCLUSION: Direct inoculation of uropathogens from Chromagar using Microscan is a reliable method of identifying uropathogens. Direct inoculation using Microscan for susceptibility testing is a reliable method except for cephalothin and piperacillin. Cefazolin can replace cephalothin as a predictor for oral cephalosporins for uncomplicated UTI.

\section{B03}

SHORT-INCUBATION-MALDI-TOF PATHOGEN IDENTIFICATION REDUCES TIME TO APPROPRIATE ANTIBIOTIC THERAPY

LDelport $^{1,2}$, A Strikwerda ${ }^{2}$, R Lannigan ${ }^{1,2}$, M John ${ }^{1}$

${ }^{1}$ London Health Sciences; ${ }^{2}$ Schulich School of Medicine, Western University, London, $\mathrm{ON}$

BACKGROUND: Sepsis accounts for significant morbidity and mortality in hospitalized patients. Approximately 30,000 Canadians develop sepsis each year with an associated mortality rate of $30 \%$. Early institution of appropriate antibiotics is associated with improved patient outcomes.

OBJECTIVES: The time to initiation of appropriate antibiotic therapy in patients with blood stream infections was compared during corresponding six week periods in 2012, 2013 to assess the impact of MALDI-TOF identification and a short incubation MALDI-TOF identification method implemented in 2014.

METHODS: As part of an on-going quality assurance program we reviewed and compared the impact of the implementation of a short incubation MALDI-TOF identification method to identify pathogens from positive blood cultures. Charts of patients with positive blood cultures were reviewed for a 6 week period in 2012 (standard incubation and ID using Vitek), 2013 (MALDI-TOF identification after standard incubation) and a 
comparable 6 week period in 2014 (short term incubation and ID using Maldi TOF). The time to initiation of appropriate antibiotic, as directed by pathogen identification was compared.

RESULTS: We identified 13/39 patients in 2012, 8/37 patients in 2013 and 10/27 patients in 2014 where empirical antibiotics needed to be changed. The average time to final identification of the pathogen was respectively 86, 74.7 and 68.6 for the respective periods. In 2014, we adapted a process whereby the interim identification obtained from the SMI was reported, this resulted in a reduction in time to initiation of more appropriate antibiotics from 73.7 hours (2012) and 62 hours (2013) to 53.75 hours in 2014.

CONCLUSIONS: We demonstrated that a rapid detection method of pathogens from positive blood cultures reduces time to initiation of appropriate antibiotics in a patient population with blood stream infections. Further studies are required to see whether this impacts patient outcomes.

\section{B04}

CHARACTERIZATION OF CANADIAN ISOLATES OF A RECENTLY-DESCRIBED GENUS AND SPECIES NOVUM, EISENBERGIELLA TAYI, OF THE FAMILY LACHNOSPIRACEAE

K Bernard $^{1}{ }^{12}$, D Wiebe ${ }^{1}$, T Burdz ${ }^{1}$, L Hoang ${ }^{3}$, P Lagacé-Wiens ${ }^{4,2}$, M Desjardins ${ }^{5}$

${ }^{1}$ National Microbiology Laboratory; ${ }^{2}$ Department of Medical Microbiology, University of Manitoba, Winnipeg, MB; ${ }^{3}$ BCCDC Public Health Microbiology \& Reference Laboratory, Vancouver, BC; ${ }^{4}$ DSM- St Boniface Hospital, Winnipeg, MB; ${ }^{5}$ University of Ottawa, Ottawa, ON

INTRODUCTION: Blood culture isolates of an unidentifiable Gram negative bacillus from patients in $\mathrm{BC}$ and $\mathrm{MB}$, referred to the NML since 2005, had been found to cocluster as a single taxon group. In 2014, Amir et al [IJSEM 64:907-914] described Eisenbergiella tayi gen. nov. sp. nov., which appeared to be consistent with these NML referrals. Here we describe features of Canadian isolates of this emerging pathogen.

METHODS: Standard methods were used for $16 \mathrm{~S}$ rRNA gene sequencing, phylogenetic analyses, phenotypic testing (conventional anaerobic biochemical testing, API 32A and BIOLOG panels) and chemotaxonomic studies (CFA composition) were done. Antimicrobial susceptibility testing (AST) was done using broth microdilution (BMD) with CLSI breakpoints for anaerobes being applied.

RESULTS: the NML analysed 8 strains from 7 patients, all from blood cultures. All were described as either Gram-stain negative or variable curvy bacilli, in contrast to Amir (2014) where these bacteria were described as Gram-negative-staining but Gram-positive structurally. All were strict anaerobes. Interestingly, some grew in the presence of bile in broth or on BBE plates, making blackened colonies (like Bilophila or Bacteroides spp). All were reactive to a variety of PY sugars / substrates; reactions for esculin, gelatin and catalase were variable. All were negative for nitrate, indole and urea. All had similar CFAs, the majority being of the saturated and mono unsaturated types. AST by the BMD method suggested that strains were susceptible to most antibiotics, including metronidazole. Phylogenetically, NML strains best fit with E. tayi in the family Lachnospiraceae, part of Clostriales Cluster XIVa.

DISCUSSION: Characterization of unusual pathogens such as these should include use of a molecular identification approach, in order to analyze novel or newly-described taxa. Pathogenicity remains unknown, but clinicians should be aware of the possibility of Eisenbergiella tayi as a cause of bacteremia.
14:45-15:45 Oral Presentations: Session C Room: Brown, Delta Prince Edward

\section{C01}

MYCOBACTERIUM ABSCESSUS GROUP SUBSPECIATION
AND CORRELATION WITH MOLECULAR AND
PHENOTYPIC DETECTION OF CLARITHROMYCIN
RESISTANCE IN ONTARIO

FB Jamieson ${ }^{1,2}$, JH Ma ${ }^{1}$, D Liu $^{3}$, DJ Farrell ${ }^{2}$

${ }^{1}$ Public Health Ontario Laboratories; ${ }^{2}$ University of Toronto,

Toronto; ${ }^{3}$ Western University, London, ON

BACKGROUND: Mycobacterium abscessus group (MabsG) are significant pathogens responsible for pulmonary infections in high risk patient groups. Mabs are resistant to many antibiotics. MabsG includes M. abscessus sensu stricto (Ma), M.massiliense (Mma), and M. bolletii (Mbo), recently confirmed "genomospecies". Previous studies identified the importance of speciation and phenotypic susceptibilities (DST) within MabsG for predicting response to therapy. We sought to correlate MabsG speciation with molecular resistance markers and DST.

METHODS: A panel of 98 clinical isolates identified as MabsG by HAIN (Nehren, Germany) Genotype CM, and the 3 type strains for Ma, Mma and Mbo were analyzed. Isolates were speciated using target specific PCR and sequencing for $h s p 65$, secAl, and erm(41), and $r r l$, for which point mutations are associated with acquired resistance to macrolides. DST was performed using RAPMYCO broth microdilution panels, read at 5, 7, 10 and 14 days (Thermo Fisher Scientific, OH).

RESULTS: 48/64 clinical Ma isolates possessed an intact erm(41) with the T28 polymorphism and were resistant, while 16 isolates had the C28 polymorphism, resulting in a non-functional erm $(41)$; of these, 9 were susceptible, 4 were intermediate, and 2 were resistant (discordant), and 1 isolate also had the $r r l$ A2059 mutation and was resistant (concordant). All (33) clinical Mma isolates had a non-functional erm(41) gene with 13 susceptible and 3 intermediate, but 17 also harbored a mutation in $r r l$, and were resistant (concordant). 1 clinical Mbo isolate had an intact erm(41) and was resistant.

CONCLUSIONS: Molecular determination of drug resistance had excellent correlation with DST. While Mma isolates have non-functional erm(41) genes and are reported to have better response to treatment, in this study, 50\% also harboured $r r l$ mutation and were resistant. A revised molecular testing algorithm is being developed.

\section{C02}

MYCOBACTERIUM TUBERCULOSIS TRENDS IN MANITOBA: 2003-2013

$\underline{\text { S Christianson }}^{1}, \mathrm{C}$ Ens $^{2}, \mathrm{~N} \mathrm{Yu}^{2}$, J Wolfe ${ }^{1}, \mathrm{M} \mathrm{Sharma}^{1}$

${ }^{1}$ Public Health Agency of Canada; ${ }^{2}$ Manitoba Health, Winnipeg, MB BACKGROUND: Despite a relatively low rate of TB in Canada, some regions in Manitoba (MB) have rates of TB that can be close to 100 times the national average. The objective of this study was to analyze both traditional and molecular epidemiology data for TB from 2003-2013 to determine trends in TB in MB.

METHODS: Retrospective analysis of 12 locus MIRU-VNTR patterns for all culture positive TB cases in MB from 2003-2013 ( $n=1429$ ) was conducted. Clusters were defined as isolates with indistinguishable MIRU. VNTR patterns. Prevalent strains were defined as clusters with $>1 \%$ of all strains. Where available, 24 locus MIRU-VNTR data and spoligotyping were used for additional cluster evaluation. Data was linked with the MB TB registry $(n=1116)$ and descriptive statistics were used to explore the demographic and geographic characteristics of clusters.

RESULTS: This analysis revealed 12 prevalent strains based on 12 locus MIRU-VNTR which accounted for $\sim 63 \%$ of all cases. Cluster 3 is consistently the most common TB type in the province each year (18.3-53.4\%). Based on the epidemiologically linked data, the majority of First Nations and Métis (FNs) TB cases belong to Clusters 1 and 3 (13\% \& 53\%). Clusters 1 and 3 also make up the majority of Canadian-born cases (28\% and 22\%). 
The majority of Foreign-born cases belong to cluster 12 (24\%). The remaining 9 clusters each account for 1.2-3.6\% of cases, with fairly consistent yearly rates. Geographic analysis of clusters among regional health authorities revealed more localized cluster variations.

CONCLUSIONS: Tuberculosis in MB is consistently dominated by three MIRU-VNTR types (clusters 1, 3 and 12). Further investigations of strain and populations genetics are required to guide public health efforts to control TB in Manitoba.

\section{C03}

\section{EVALUATION OF A LATERAL FLOW IMMUNOASSAY FOR CULTURE CONFIRMATION OF MYCOBACTERIUM TUBERCULOSIS COMPLEX}

$\mathrm{M} \mathrm{Ng}, \mathrm{M}$ Rodrigues, $\mathrm{P}$ Tang

BC Public Health Microbiology \& Reference Laboratory, Vancouver, BC BACKGROUND: Potential isolates of M. tuberculosis complex (MTBC) must be identified rapidly for TB patient care and control. The typical method for identification of MTBC isolates is a non-amplified DNA probe test which requires a significant amount of growth and sometimes fails to detect cultures at early stages of growth. SD Bioline (South Korea) has developed a rapid immunochromatographic test (ICT) kit that claims to have excellent sensitivity and specificity, and is not expensive. This kit has been demonstrated to have a detection limit of $10^{5} \mathrm{CFU} / \mathrm{mL}$ and can be used as soon as tiny colonies on solid media are observed or when the auto-detection of positivity is detected in a liquid culture system. We tested this ICT against a standard DNA probe test (Gen-Probe AccuProbe MTBC).

METHODS: Positive cultures from Mycobacterium Growth Indicator Tubes (MGITs, BD Biosciences) or Lowenstein-Jensen pyruvate agar or $\mathrm{Myco} / \mathrm{F}$ Lytic blood culture vials (BD Biosciences) were used. After smearing each culture, an aliquot was tested with the ICT and the DNA probe test.

RESULTS: Out of 86 isolates tested, 52 were MTBC and the rest were NTMs. The ICT specificity was $94 \%$, and the sensitivity was $94 \%$ when compared to the DNA probe test. The reduced specificity was due to the DNA probe missing two MTBC-positive samples that were detected by the ICT: one due to not enough growth and the other due to the presence of blood. The reduced sensitivity was due to the ICT missing 3 DNA probe positives. The first discrepant isolate was M. bovis BCG which is appropriately negative by the ICT since it detects Mpt64 antigen. The second discrepant isolate was suspected to have a mutation in mpt64: the gene was detectable by PCR but not by ICT even upon subculturing in different media. The third discrepant isolate was from a lymph node sample that was submitted in an unidentified pink fluid. When an aliquot of the culture media was heat killed in preparation for a confirmatory TB PCR assay, it produced a white precipitate. Subculturing the isolate allowed the ICT to become positive. CONCLUSIONS: Our results are in agreement with the literature for this ICT. The ICT was simple to use. The rapid identification of MTBC in fifteen minutes without any equipment costs (versus three hours using the Gen-Probe AccuProbe) will not only reduce the culture identification workload but also will be cost efficient while improving turn-around times.

\section{C04}

UNDERSTANDING PSEUDOMONAS AERUGINOSA TREATMENT RESISTANCE IN CHRONIC LUNG INFECTION ISING DEEP POPULATION SEQUENCING

B Coburn ${ }^{1}$, J Diaz Caballero' ${ }^{1}$, S Clark ${ }^{1,2}$, P Wang ${ }^{1}$, S Donaldson ${ }^{1}$, Y Zhang ${ }^{2,3}$, Y Yau ${ }^{1,4}$, V Waters ${ }^{1,4}$, DE Tullis ${ }^{1,5}$, D Hwang ${ }^{2,3}$, D Guttman ${ }^{1}$

${ }^{1}$ University of Toronto; ${ }^{2}$ University Health Network;

${ }^{3}$ Toronto General Research Institute; ${ }^{4}$ Hospital for Sick Children;

${ }^{5}$ St Michael's Hospital, Toronto, ON

The opportunistic human pathogen Pseudomonas aeruginosa is highly resistant to eradication once established in the lungs of cystic fibrosis (CF) patients. While the clinical implications of these infections are well studied, we know little about the evolutionary processes that enable this pathogen to persist in this environment.

Here, we describe the longitudinal analysis of a single CF patient chronically infected with $P$. aeruginosa. Using culture-independent techniques
(16S rRNA gene sequencing) we show that $P$. aeruginosa is the dominant bacterial constituent of the lung, despite numerous courses of multiple anti-pseudomonal antibiotics. We then whole-genome sequenced a total of $235 \mathrm{P}$. aeruginosa isolates twelve sputum specimens obtained over a oneyear period. This revealed multiple intraspecies 'selective sweeps', demonstrating that within species population dynamics result in periodic complete or nearly complete strain replacement, producing multiple distinct populations: an ancestral population characterized by higher diversity and recombination, and a sweep population characterized by mutations in the gene encoding penicillin-binding protein 3 (PBP3). These mutations correlate with resistance to anti-pseudomonal antibiotics. These intraspecies dynamics are not apparent with lower resolution analyses, and demonstrate an adaptive mechanism allowing $P$. aeruginosa to persist in the $\mathrm{CF}$ lung during therapy.

\section{4:45-15:45 Oral Presentations: Session D} Room: Chandler, Delta Prince Edward

\section{D01}

\section{PREVALENCE OF HAEMOPHILUS INFLUENZAE TYPE A IN REMOTE FIRST NATIONS COMMUNITIES OF NORTHWESTERN ONTARIO}

M Ulanova ${ }^{1}$, E Nix ${ }^{1}$, RSW Tsang ${ }^{2}$, W McCready ${ }^{1}$

${ }^{1}$ Northern Ontario School of Medicine, Thunder Bay, ON; ${ }^{2}$ National Microbiology Laboratory, Winnipeg, MB

BACKGROUND: High incidence of severe invasive infections caused by Haemophilus influenzae type a (Hia) has been reported from Northwestern Ontario. Most cases struck young First Nations children or adults with significant underlying medical conditions. Although the cases were epidemiologically unrelated and often occurred in remote communities all the Hia isolates were remarkably similar by their molecular-genetic characteristics. We hypothesized that the persistent circulation of Hia in First Nations communities is an underlying reason for increased rates of invasive disease in this population.

OBJECTIVE: To characterize $H$. influenzae isolates in an area containing 28 First Nations communities, most of which are not accessible by road. METHODS: All H. influenzae isolates from sick individuals were collected by the clinical laboratory in one Aboriginal Health Centre during November 2013 - October 2014. Identification of H. influenzae was confirmed by $16 \mathrm{~S}$ ribosomal RNA sequencing; serotyping was performed by both bacterial agglutination test and PCR to detect the serotype-specific genes. Clonal analysis and detection of the IS1016-bexA partial deletion in the capsular loci were carried out by multilocus sequence typing and PCR, respectively.

RESULTS: Forty-one (39 non-invasive and 2 invasive) H. influenzae isolates were identified from 41 First Nations individuals aged 1-39 years. The majority of isolates (33) were non-typeable strains from the ear, eye, or sputum. Hia represented $10 \%$ of non-invasive isolates (4 isolates from the ear in young children) and $50 \%$ of invasive isolates (one blood isolate from an adult). Among other encapsulated isolates, there were two Hif (from eye and ear) and one Hie (from blood); no Hib was detected.

CONCLUSIONS: Beyond being a significant cause of invasive disease in First Nations communities of Northwestern Ontario, Hia is also frequently isolated from young children with ear infection. Our findings suggest that continuing circulation of this pathogen in First Nations communities is a likely reason for the occurrence of invasive Hia disease in vulnerable population groups. Given the success of conjugated Hib vaccine in decreasing the rates of invasive Hib disease and Hib carriage, paediatric immunization against Hia can be similarly successful in preventing infections caused by Hia. 


\section{D02}

SEROTYPE DISTRIBUTION AND ANTIMICROBIAL SUSCEPTIBILITY OF INVASIVE AND NONINVASIVE STREPTOCOCCUS PNEUMONIAE IN CANADA: CANWARD 2007-2013

AR Golden ${ }^{1}$, HJ Adam ${ }^{1,2}$, M Baxter ${ }^{1}$, KA Nichol' ${ }^{2}$, I Martin ${ }^{3}$, W Demczuk $^{3}$, M Gilmour ${ }^{1,2}$, JA Karlowsky ${ }^{1,2}$, DJ Hoban ${ }^{1,2}$, GG Zhanel ${ }^{1}$

${ }^{1}$ University of Manitoba; ${ }^{2}$ Diagnostic Services Manitoba;

${ }^{3}$ Public Health Agency of Canada - National Microbiology Laboratory, Winnipeg, MB

OBJECTIVES: The goal of this study was to determine the serotype distributions and antimicrobial susceptibilities of Streptococcus pneumoniae collected from blood and respiratory infections across Canada in 2007-2013.

METHODS: S. pneumoniae strains were obtained from Canadian hospitals as part of the ongoing national surveillance study, CANWARD. S. pneumoniae were serotyped using the Quellung method. Antimicrobial susceptibility testing was performed using the CLSI broth microdilution method. Multi-drug resistance (MDR) was defined as resistance to $\geq 3$ structurally unrelated classes of antimicrobials.

RESULTS: 2,154 S. pneumoniae isolates were collected during the CANWARD 2007-2013 study; 1,386 (64\%) were obtained from respiratory samples and $768(36 \%)$ from blood samples. The 10 most common serotypes from respiratory isolates were 3, 11A, 19A, 22F, 19F, 23A, non-typeable, 6C, 35B and 23B. The 10 most common serotypes from blood were 19A, 3, 22F, 7F, 4, 5, 12F, 11A, 8 and 9N. Antimicrobial susceptibilities were: 78\% (respiratory isolates)/80\% (blood isolates) for clarithromycin (CLR), $92 / 94 \%$ for clindamycin (CLD), 85/90\% for doxycycline (DOX), 99/99\% for levofloxacin, $82 / 88 \%$ for penicillin (PEN, oral penicillin V breakpoints), $84 / 88 \%$ for trimethoprim-sulfamethoxazole (SXT) and $99 / 100 \%$ for ceftriaxone (nonmeningitis breakpoints). Isolates from blood demonstrated 4.4\% MDR, primarily to CLR, CLD, DOX, PEN and SXT in serotype 19A. A variety of MDR patterns were observed in $8.6 \%$ of respiratory isolates, predominantly in serotypes $15 \mathrm{~A}, 19 \mathrm{~A}, 19 \mathrm{~F}$ and non-typeable isolates. MDR in respiratory isolates was statistically significantly higher than in blood isolates $(\mathrm{P}<0.001)$. CONCLUSIONS: The top $10 \mathrm{~S}$. pneumoniae serotypes causing respiratory and blood infections in Canada in 2007-2013 were significantly different, with the exception of 3,11A, 19A and 22F. S. pneumoniae isolated from respiratory samples demonstrated lower antimicrobial susceptibilities and higher MDR in a wider range of serotypes than S. pneumoniae isolated from blood.

\section{D03}

ANTIBIOTIC SUSCEPTIBILITY OF INVASIVE HAEMOPHILUS INFLUENZAE STRAINS IN CANADA, 2007 TO JUNE 30, 2014 K Whyte, T Drew, D Krentz, M Shuel, RSW Tsang

Vaccine Preventable Bacterial Diseases, National Microbiology

Laboratory, Public Health Agency of Canada, Winnipeg, MB

OBJECTIVE: Susceptibility of invasive Haemophilus influenzae (Hi) to commonly prescribed antibiotics was determined. Canadian isolates from 2007-2014 were included and they were also characterized based on their serotype and sequence type, to determine any possible correlation with susceptibility profiles.

METHODS: Standard methods were used to determine serotype and MLST. $\beta$-lactamase was determined by chromogenic method and amplification of TEM-1 and ROB-1 genes. Disk diffusion and MIC determination were conducted according to CLSI guidelines.

RESULTS: Of the 849 invasive isolates examined, resistance to one or more antibiotics was seen in $48.0 \%$ of non-typeable (229/477), 36.4\% of serotype b (24/66), 33.3\% of serotype e (7/21), 16.7\% of serotype c (1/6), $14.0 \%$ of serotype $f(13 / 93)$ and $3.2 \%$ of serotype a (6/186) isolates. $\beta$-lactamase mediated ampicillin resistance was most commonly observed among all serotypes and non-typeable isolates, with rates as high as $30.3 \%$ of the serotype b isolates (20/66) and $21.6 \%$ of the non-typeable isolates (103/477). Resistance to trimethoprim-sulfamethoxazole was observed with rates as high as $20.8 \%$ of non-typeable $(99 / 477)$ and $13.6 \%$ of serotype b (9/66) isolates. Resistance or reduced susceptibility to a number of other antibiotics was also observed. Multidrug resistance was observed in serotype a, b, c and non-typeable isolates.

CONCLUSIONS: Overall, higher incidence of resistance was observed with non-typeable isolates compared to serotypeable isolates. Resistance rates of non-typeable isolates were also higher for specific antibiotics, with the exception of ampicillin resistance in serotype b isolates. Non-typeable isolates show resistance to more antibiotics, and higher incidence of multidrug resistance compared to serotypeable isolates.

\section{D04}

\section{DIFFERING TRENDS IN ANTIBIOTIC PRESCRIBING BY PROFESSION}

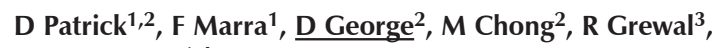
E Blondel-Hill ${ }^{1,4}$

${ }^{1}$ University of British Columbia; ${ }^{2}$ British Columbia Centre for Disease Control; ${ }^{3}$ BC Dental Association, Vancouver; ${ }^{4}$ Interior Health Authority, Kelowna, BC

OBJECTIVES: In recent years, legislation allows several healthcare professions to prescribe antimicrobials. In British Columbia, midwives began prescribing in 1998, nurse-practitioners in 2005, optometrists in 2009 and naturopathic doctors (NDs) in 2010. We aimed to describe trends in prescribing by profession to futher inform stewardship efforts.

METHODS: All outpatient prescriptions in British Columbia, Canada, are entered in the British Columbia Pharmanet database. We obtained anonymized, line-listed data on prescriptions from 1996-2013, including a variable coding for licencing body of the prescriber. Analyses were conducted in SAS and Excel using Anatomical Therapeutic Classification (ATC) standard codes and defined daily dose values. Rates of prescribing were normalized to the British Columbia population and expressed in defined daily doses per 1000 persons per day (DID).

RESULTS: In 2013, physicians wrote $87.37 \%$ of prescriptions, dentists $11.25 \%$, nurses-practitioners $0.59 \%$, pharmacists $0.38 \%$, podiatrists $0.10 \%$, midwives $0.04 \%$, naturopaths $0.10 \%$ and others $0.17 \%$. From $1996-2013$, overall antibiotic use declined from 18.3 to 16.0 DID and physician use declined $18.2 \%$ from 17.35 to 14.11 DID. However, dental surgeons increased their rate $62.2 \%$ from 0.99 to 1.59 DID. Dental surgeons used mostly beta-lactams, especially amoxicillin without a beta-lactamase inhibitor. The mean number of DDD issued with each antibiotic prescription was 8.4 for midwives, 9.3 for dental surgeons, 9.7 for nurse practitioners, 10.6 for physicians and 35.2 for ND's in 2013. ND's made predominant use of tetracyclines and macrolides.

CONCLUSIONS: While physician prescribing has been driving an overall decline in antibiotic utilization in British Columbia, it will be important to address stewardship efforts to all prescribing professions with an initial focus on dentistry. While NDs contribute only a small proportion of prescriptions, a DDD/prescription rate more than three times that of other professions requires an explanation and may indicate a need for continuing education efforts in antibiotic use.

16:00-17:30 Oral Presentations: Session E Room: Henry, Delta Prince Edward

\section{E01}

\section{NURSING ADVANCED DIRECTIVE TO REMOVE URINARY CATHETERS AMONG GENERAL INTERNAL MEDICINE PATIENTS: A CONTROLLED BEFORE-AFTER STUDY}

C Corpus ${ }^{1}$, B Catt ${ }^{1}$, P Cho ${ }^{1}$, F Wong ${ }^{1}$, S Callery ${ }^{1}$, B Wong ${ }^{1,2,3}$, A Simor ${ }^{1,2,4}, \mathrm{M}_{\text {Vearncombe }}{ }^{1,2,4}$, |A Leis ${ }^{1,2,3}$

${ }^{1}$ Sunnybrook Health Sciences Centre; ${ }^{2}$ University of Toronto; ${ }^{3}$ Centre for Quality Improvement and Patient Safety and Department of Medicine; ${ }^{4}$ Department of Laboratory Medicine and Pathobiology, University of Toronto, Toronto, ON BACKGROUND: Overuse of urinary catheters among general medical inpatients contributes to increased incidence of healthcare-associated urinary tract infection and catheter-associated asymptomatic bacteriuria. Prior studies 
suggest physicians are often unaware of whether patients are catheterized, and nurse-led directives for catheter removal can reduce unnecessary use.

METHODS: An advanced directive was implemented allowing nurses to remove urinary catheters for patients admitted to two general medical units who lacked the following acceptable indications: pre-admission permanent indwelling catheter, bladder outlet obstruction, need for continuous bladder irrigation, stage 3/4 sacral ulcers among incontinent patients, patient wishes for comfort care in end of life, or serum sodium $<120 \mathrm{mmol} / \mathrm{L}$ with a physician order for strict fluid monitoring. Catheterdays per patient-days was compared monthly to two control general medical units before ( 1 month) and following ( 3 months) implementation using a task-oriented nurse acuity tool validated to measure urinary catheter use. Active surveillance for catheter re-insertion within 48-hours was performed with chart reviews to assess for re-insertion due to inappropriate urinary catheter removal.

RESULTS: At baseline, urinary catheter-days per patient-days were 252/1332 (18.9\%; 95\% CI, 16.9-21.1\%) and 264/1410 (18.7\%; 95\% CI, $16.8-20.8 \%)$, on intervention and control units, respectively $(\mathrm{P}=0.89)$. Following implementation of nursing advanced directive, catheter-days per patient-days on intervention units decreased to $107 / 1351$ (7.9\%; 95\% CI: 6.6-9.5\%), significantly below control units (194/1539, 12.6\%; 95\% CI: $11.0-14.4 \%, \mathrm{P}=0.0001)$. Three-months after implementation, catheter use on intervention units remained significantly lower $(96 / 1564,6.1 \%$; 95\% CI: $5.1-7.4 \%)$ compared to control units (254/1669, 15.2\%; $95 \%$ CI: $13.6-17.1 \%, \mathrm{P}<0.002)$. Eight catheter re-insertions occurred over 3 -months, and none were related to inappropriate catheter removal.

CONCLUSIONS: A nursing advanced directive to remove unnecessary urinary catheters on general medical units led to a significant reduction in urinary catheter use without inappropriate removal. Long-term follow-up is needed to confirm sustainability of this model of care.

\section{E02}

\section{IMPACT OF URINARY TRACT INFECTION PROVINCIAL EMPIRIC TREATMENT GUIDELINES ON PRESCRIBING OF NITROFURANTOIN IN PRIMARY CARE AND LONG TERM CARE SETTINGS IN THE PROVINCE OF PRINCE EDWARD ISLAND}

¿ Boswell ${ }^{1}$, S Chuu ${ }^{1}$, W Cooke ${ }^{2}$, I Ferrish ${ }^{3}$, G German ${ }^{1}$

${ }^{1}$ Health PEI; ${ }^{2}$ Queen Elizabeth Hospital, Charlottetown;

${ }^{3}$ Prince County Hospital, Summerside, PE

OBJECTIVES: The Health PEI Urinary Tract Infection Empiric Treatment Guidelines were distributed to all physicians, nurse practitioners, and pharmacists in Prince Edward Island on May 24, 2013. In these provincial guidelines, nitrofurantoin is recommended as the first-line option for early cystitis in females (if no contraindication for its use) and a twice daily dosing formulation (MacroBIDR) is suggested over four times daily dosing formulations to improve patient adherence. The objective of this audit is to examine the potential impact the guidelines have on nitrofurantoin prescribing practices in primary care and long term care settings.

METHODS: Information on nitrofurantoin prescriptions dispensed at community pharmacies and Provincial Pharmacy from January 1, 2012 to October 28, 2014 was obtained from the provincial Drug Information System. Prescriber claims using ICD-9 diagnosis codes for UTI-related diagnoses were retrieved for females from the Medicare Claims System. The pre-intervention period was defined as January 2012 to November 2012 and the post-intervention period was June 2013 to October 2014

RESULTS: When comparing the pre-intervention period to the postintervention period, the average number of monthly nitrofurantoin prescriptions dispensed for female patients increased by $30.7 \%(\mathrm{P}<0.001)$. As a potential confounder, the number of diagnoses of UTIs pre- and postintervention was examined. The average number of monthly female UTI claims increased post-intervention, although not significantly $(+7.6 \%$, $\mathrm{P}=0.06)$. When controlled for differences in UTI diagnosis claims, the percent of nitrofurantoin prescriptions increased by $21.6 \%(\mathrm{P}<0.001)$. The percentage of nitrofurantoin prescriptions for MacroBID® increased by $25.7 \%$ in the post-intervention period $(\mathrm{P}<0.001)$.

CONCLUSIONS: Primary care clinicians are more likely to prescribe nitrofurantoin for female UTIs after the release of the guidelines and this increase was sustained over the post-intervention period. The new guidelines appear to have a positive and lasting impact on the prescribing behaviours of PEI prescribers.

\section{E03}

EARLY ONSET INVASIVE CANDIDIASIS IN EXTREMELY LOW BIRTH WEIGHT INFANTS: RISKS AND OUTCOMES IN A MULTICENTRE CANADIAN PROSPECTIVE STUDY

M Barton ${ }^{1}$, YH Shen ${ }^{2}, K^{\prime} \mathrm{O}^{\prime}$ Brien $^{3}$, J Robinson ${ }^{4}$, HD Davies ${ }^{5}$, K Simpson $^{2}$, E Asztalos ${ }^{6}$, J Langley ${ }^{7}$, N Le Saux ${ }^{8}$, R Sauve ${ }^{5}$, A Synnes ${ }^{9}$, B Tan ${ }^{10}$, L De Repentigny ${ }^{11}$, E Rubin ${ }^{12}$, C Hui ${ }^{13}$, L Kovacs ${ }^{14}, Y_{Y^{2}}{ }^{2}$, S Richardson ${ }^{2}$

${ }^{1}$ Western University, London; ${ }^{2}$ The Hospital for Sick Children; ${ }^{3}$ Mount Sinai Hospital, Toronto, ON; ${ }^{4}$ Stollery Children's Hospital, Edmonton; ${ }^{5}$ Foothills Hospital, Calgary, AB; ${ }^{6}$ Sunnybrook Health Sciences Centre, Toronto, ON; ${ }^{7}$ Dalhousie University, Halifax, NS; ${ }^{8}$ Children's Hospital of Eastern Ontario, Ottawa, ON; ${ }^{9}$ British Columbia's Women's Hospital, Vancouver, BC; ${ }^{10}$ Royal University Hospital, Saskatoon, SK; ${ }^{11} \mathrm{CHU}$ Sainte-Justine; ${ }^{12}$ Montreal Children's Hospital, Montreal, QC; ${ }^{13}$ McMaster University, Hamilton, ON; ${ }^{14}$ Jewish General Hospital, Montreal, QC

BACKGROUND: Data on neonatal invasive candidiasis (IC) presenting in the first week of life are limited to case reports. This entity is less well described than later onset IC. We sought to compare the clinical features and outcome in early onset disease (EOD, $<=7 \mathrm{~d}$ ) with those in late onset disease (LOD, $>7$ d) in extremely low birth weight (ELBW, $<1000 \mathrm{~g}$ ) infants.

METHODS: All ELBW cases and their controls (2 controls: 1 case) from a Canadian multicentre prospective study of neonatal candidiasis in very low birth weight infants were selected for secondary analysis. Factors associated with occurrence and outcome of EOD in ELBW infants were identified using logistic regression.

RESULTS: EOD occurred in 14 of 45 (31\%) ELBW infants with IC compared to $2 / 24$ (8\%) of infants $\geq 1000 \mathrm{~g}(\mathrm{P}=0.039)$. Birth weight $<750 \mathrm{~g}$, gestation $<25$ weeks, chorioamnionitis and vaginal delivery were all associated with EOD $(\mathrm{P}<0.01)$ versus LOD. Infection with Candida albicans, disseminated disease, pulmonary involvement and cardiovascular disease were significantly more common in EOD than in LOD $(\mathrm{P}<0.05)$. The EOD case fatality rate (71\%) was higher than in LOD (32\%) or controls (15\%); $\mathrm{P}=0.0001$. The rate of neurodevelopmental impairment and mortality combined was similar in EOD $(86 \%)$ and LOD (72\%) but higher than in controls $(32 \% ; \mathrm{P}=0.007)$.

CONCLUSION: Outcomes of EOD in ELBW infants are poor. The association of EOD with chorioamnionitis, vaginal delivery and pneumonia support perinatal acquisition. Dissemination is common and affected infants often die. Empiric antifungal treatment should be considered for ELBW infants with pneumonia, delivered vaginally, whose mothers have chorioamnionitis or an intrauterine foreign body.

\section{E04}

SUCCESSFUL TREATMENT OF CANDIDA ALBICANS MENINGITIS WITH INTRATHECAL AMPHOTERICIN AND INTRAVENOUS FLUCONAZOLE: A CASE REPORT AND REVIEW OF LITERATURE

B Ghadaki ${ }^{1}$, N Irfan ${ }^{2}$, M Loeb $^{3}$

${ }^{1}$ McMaster University; ${ }^{2}$ Hamilton Health Sciences; ${ }^{3}$ Department of Pathology and Molecular Medicine, McMaster University, Hamilton, ON

OBJECTIVE: To present a case of Candida albicans meningitis post craniopharyngioma resection successfully treated with a combination of intrathecal (IT) amphotericin and intravenous (IV) fluconazole.

METHODS: A case report and literature review (1958-2014) on the treatment of Candida albicans meningitis complicating neurosurgical procedures was performed via Pubmed and Ovid using search terms: "Candida", "meningitis", "shunt-infection", "neurosurgical", and "treatment".

RESULTS: A 59-year-old man underwent resection of a craniopharyngioma complicated by a CSF leak necessitating lumboperitoneal (LP) shunt insertion. Postoperatively he developed persistent fever and a reduced level of consciousness. CSF analysis revealed elevated leucocytosis and protein with 
normal glucose. He continued to be febrile while on broad-spectrum antibiotics. Although initial blood and CSF cultures were negative, following removal of LP shunt both CSF and shunt tip grew Candida albicans. After the initiation of IV amphotericin, the patient quickly defervesced with a decrease in CSF leucocytosis. His course was then complicated by acute renal failure requiring a switch to IV fluconazole. After 7 days of therapy his level of consciousness worsened with increased CSF leucocytosis. However, cultures remained negative. IT amphotericin was added and the patient improved clinically and biochemically. Review of the literature revealed 85 cases of Candida infection complicating neurosurgery for which the majority were secondary to Candida albicans. Over $85 \%$ of cases received IV amphotericin alone or in combination with IT amphotericin, flucytosine and/or fluconazole. No cases reported use of IT amphotericin with IV fluconazole. Cure rates with IV amphotericin with or without IT amphotericin were highest and ranged from 80 to $90 \%$, while fluconazole monotherapy resulted in a cure rate of $60 \%$.

CONCLUSION: Candida infections of the central nervous system are an increasingly recognized post-neurosurgical complication associated with significant mortality. Patients that do not tolerate IV amphotericin may benefit from the addition of IT amphotericin to fluconazole.

\section{E05}

\section{LINEZOLID-RESISTANT MRSA STRAINS SEQUENTIALLY ISOLATED FROM AN ELDERLY PATIENT WITH PNEUMONIA DURING PROLONGED THERAPY WITH LINEZOLID \\ X-K Hao \\ Fourth Military Medical University, Xi'an, China}

OBJECTIVE: Linezolid is a crucial drug for methicillin-resistant Staphylococcus aureus (MRSA) infection. Here we describe the emergence of linezolid-resistant MRSA strains with a 23S rRNA mutation sequentially isolated from an elderly patient with pneumonia, during longterm linezolid treatment.

METHODS: A retrospective analysis was conducted to calculate the cumulative amount of linezolid administered to an elderly patient with pneumonia during prolonged therapy with linezolid. The sequential isolates were identified and their minimum inhibitory concentration (MIC), homology and drug resistant related genes (cfr, 23S rRNA) were investigated.

RESULTS: One linezolid-resistant MRSA strain was isolated from sputum of the patient at Xijing Hospital. The MIC of linezolid had increased from $1.5 \mu \mathrm{g} / \mathrm{mL}$ of initial strain 083 in March 2012 to subsequent $4.0 \mu \mathrm{g} / \mathrm{mL}$ of strain 158 in November 2012 and $16.0 \mu \mathrm{g} / \mathrm{mL}$ of strain 231 in March 2013 respectively. The increasing MIC values to linezolid were directly correlated with the cumulative amount of linezolid $118.8 \mathrm{~g}$ administered over 99 days. Strain 158 had heterogeneous resistance to linezolid. Molecular typing showed that three MRSA strains belonged to MLST ST-239 (spa t-030) with $98.8 \%$ homology. Cfr mutation was not detected but 23S rRNA mutation G2603T was confirmed in strains 158 and 231.

CONCLUSIONS: This is the first case of linezolid-resistant MRSA in China. The long-term use of linezolid for the treatment of MRSA infection was associated with a 23S rRNA G2603T mutation, which mediates heterogeneous resistance to linezolid. And the strain with heterogeneous resistance might progress to a high-level linezolid-resistant strain when reexposed to linezolid.

\section{E06}

COMPARISON OF OUTCOMES BETWEEN VRE AND VSE BACTEREMIA IN THE ERA OF EFFECTIVE VRE THERAPY: A SYSTEMATIC REVIEW AND META-ANALYSIS

C Prematunge ${ }^{1}$, C McDougall ${ }^{1}$, J Johnstone ${ }^{1,2,3}$, K Adomako ${ }^{1}$, F Lam ${ }^{1}$, J Robertson ${ }^{1}$, G Garber $1,3,4,5$

${ }^{1}$ Public Health Ontario, Infection Prevention and Control;

${ }^{2}$ St Joseph's Health Centre; ${ }^{3}$ Department of Medicine, University of Toronto, Toronto; ${ }^{4}$ Department of Medicine, University of Ottawa; ${ }^{5}$ Ottawa Hospital Research Institute, Ottawa, ON

OBJECTIVES: Prior data suggests that vancomycin-resistant enterococcus (VRE) bacteremia is associated with worse outcomes when compared to vancomycin-sensitive enterococcus (VSE) bacteremia. However, many studies evaluating outcomes were conducted prior to the availability of effective VRE therapy. Thus, we aimed to systematically review all studies comparing outcomes of VRE bacteremia to VSE bacteremia following the advent of effective VRE therapies.

METHODS: Electronic databases and grey literature published between January 1997 and December 2014 were searched to identify all primary research studies comparing outcomes of VRE and VSE bacteremias among hospital patients, following the availability of effective VRE therapies. The primary outcome was all-cause, in-hospital mortality and total hospital length of stay (LOS) was a secondary outcome of interest. All meta-analyses were conducted in RevMan5.3 using inverse variance, random effects modelling.

RESULTS: Overall, twelve observational studies, representing 685 VRE and 1257 VSE bacteremia cases, were included in the review; eleven were cohort studies and one was a case control. All studies were conducted between September 1998 and December 2011. VRE bacteremia was associated with an increased risk of death when compared to VSE bacteremia with no heterogeneity when cohort study data was pooled in unadjusted analysis (odds ratio 1.80 [95\% CI 1.38-2.35], I2 0\%, $\mathrm{n}=11$ ) and adjusted analysis (hazards ratio 1.12 [95\% CI 1.08-1.16], $\mathrm{I}^{2}=0 \%, \mathrm{n}=2$ ). The unadjusted estimate from the single case-control study was similar, but not statistically significant (odds ratio 1.93 [95\% CI 0.97-3.82]). VRE bacteremia was associated with a longer hospital LOS than VSE bacteremia (LOS mean difference 4.00 [95\% CI -1.95-9.96], $\left.I^{2}=67 \%, n=4\right)$, but results were not statistically significant and demonstrated substantial heterogeneity. CONCLUSIONS: Despite the use of effective VRE therapy, VRE bacteremia remains associated with an increased risk of in-hospital mortality when compared to VSE bacteremia

16:00-17:30 Oral Presentations: Session F Room: Johnson, Delta Prince Edward

\section{F01}

\section{IMPLEMENTATION OF SIEMENS' CENTAUR SYPHILIS} ENZYME IMMUNOASSAY: BC EXPERIENCE

A Mak ${ }^{1}$, Y Simpson ${ }^{1}$, N Chahil ${ }^{1}$, K Chu ${ }^{1}$, Q Wong ${ }^{1}$, M Morshed ${ }^{1,2}$ ${ }^{1}$ Public Health and Reference Laboratory; ${ }^{2}$ UBC Department of Pathology and Lab Medicine, Vancouver, BC

OBJECTIVE: To examine syphilis screen false positive tests after implementation of Siemens' Centaur Syphilis Enzyme Immunoassay (TPE). METHODS: Public Health Microbiology and Reference Laboratory implemented the Siemens' Centaur Syphilis EIA assay on July 9, 2014 as the screening test. Samples are initially tested on the Siemens' automated line using the Centaur Syphilis EIA assay and samples found to be equivocal or reactive are further tested by RPR, TPPA and INNO-LIA.

RESULTS: A total of 105,934 samples were tested using Siemens' Syphilis EIA assay from July 9 to Dec 31, 2014. 101,709 (96\%) samples tested as nonreactive, $4,155(3.9 \%)$ tested as reactive and $70(<0.1 \%)$ as equivocal. $248(0.2 \%)$ patients with potential false positive TPE results (had nonreactive confirmatory tests) were retested on a follow up sample. The potential TPE false positive patients demonstrated TPE index values ranging from $>1.0$ to 11.17. The clinical information for these potential false positive patients needs to be further investigated. False positive rates for the RPR screening test have been reported to be up to $2.0 \%$ or greater.

CONCLUSIONS: Preliminary assessment of potential false positive TPE index values ranged from $>1.0$ to 11.17 . Index value may be an indicator of a false positive syphilis EIA test, but further clinical correlation is needed. The false positive rate for Siemens Centaur TPE is lower than for RPR. 
F02

GENETIC DIVERSITY AND MOLECULAR EVOLUTION OF RESPIRATORY SYNCYTIAL VIRUS A ON1 GENOTYPE, 20102013: A COMPARATIVE ANALYSIS

A Granados ${ }^{1,2}$, VR Duvvuri ${ }^{1,3}$, P Rosenfeld ${ }^{1}$, A Eshaghi ${ }^{1}$, [B Gubbay ${ }^{1}, 2$ ${ }^{1}$ Public Health Ontario; ${ }^{2}$ University of Toronto, Toronto; ${ }^{3}$ University of Waterloo, Waterloo, ON

In 2010, a novel genotype of human respiratory syncytial virus (RSV), RSVA ON1, was first detected in Ontario, Canada, and subsequently isolated from various countries. In order to investigate its persistence and dynamics, we tested 406 randomly selected RSV positive samples submitted to Public Health Ontario from August 2011 to August 2012 by realtime RT-PCR. We identified RSVA NA1 ( $n=181)$, NA2 ( $n=135)$, GA5 $(n=3)$, and ON1 $(n=51)$, and RSV-B $(n=36)$. In addition to the 51 ON1 samples identified, we also collected $346 \mathrm{ON} 1 \mathrm{G}$-gene sequences from 18 countries from GenBank to evaluate ON1 genetic diversity, lineage distribution, rate of evolution, selection pressure, and the time of most recent common ancestor (tMRCA). Phylogenetic analysis determined that globally there are three ON1 lineages and we re-confirmed a newly defined genotype, ON2, recently reported in Italy. In Ontario there are two ON1 lineages circulating concurrently with a new cluster that has agreement with lineage identification criteria. ON1 is evolving at a rate of $3.29 \times 10^{-3}$ substitutions/site/year. Path-O-Gen analysis determined the possible date of tMRCA as 2002. Selection pressure analysis identified 3 positively selected sites: V225, L274, and Y297 that are heavily selected ( $\mathrm{dN} / \mathrm{dS}=3.5)$ in the global G-genes, whereas only $1(\mathrm{~V} 303 ; \mathrm{dN} / \mathrm{dS}=13.5)$ was identified in Ontario. This study demonstrated that HRSV A-ON1 is rapidly evolving and spreading globally.

\section{F03}

\section{SHEDDING OF LIVE-ATTENUATED INFLUENZA VACCINE (LAIV) VIRUSES IN CANADIAN CHILDREN AND ADOLESCENTS WITH AND WITHOUT CYSTIC FIBROSIS}

$\underline{\text { C Boikos }}{ }^{1}$, H Charest ${ }^{2}$, J Papenburg ${ }^{3}$, D Scheifele ${ }^{4,5}$, LC Lands ${ }^{6}$, G Deserres $^{7,8}$, C Quach ${ }^{1,8,9,10}$

${ }^{1}$ Department of Epidemiology, Biostatistics \& Occupational Health, McGill University, Montreal; ${ }^{2}$ Laboratoire de santé publique du Québec/Institut national de santé publique du Québec, Sainte-Annede-Bellevue, QC; ${ }^{3}$ Department of Pediatrics, Division of Infectious Diseases, The Montreal Children's Hospital, McGill University, Montreal, QC; ${ }^{4}$ Division of Infectious and Immunological Diseases, Department of Pediatrics, University of British Columbia; ${ }^{5}$ Vaccine Evaluation Center, Child \& Family Research Institute, Vancouver, BC; ${ }^{6}$ Department of Pediatrics, Division of Respiratory Medicine, The Montreal Children's Hospital, McGill University, Montreal;

${ }^{7}$ Department of Social and Preventive Medicine, Laval University; ${ }^{8}$ Institut national de santé publique du Québec, Québec;

${ }^{9}$ Department of Pediatrics, Division of Infectious Diseases, Centre Mère-Enfant Soleil du CHU de Québec, Québec; ${ }^{10}$ McGill University Health Centre, Vaccine Study Centre, Research Institute of the MUHC, Montreal, QC

OBJECTIVE: To quantify and describe trends in shedding of vaccine-strain influenza viruses following administration of LAIV in Canadian children and adolescents aged 2-19 years with and without cystic fibrosis (CF).

METHODS: During the 2013-14 influenza season, 76 participants (57 patients with CF and 19 healthy siblings) were followed-up for 7 days post-LAIV administration. Reverse-transcription polymerase chain reaction (RT-PCR) and viral cultures were performed on flocked nasal swabs obtained from each participant immediately before vaccination (day 0 ) and on days 1, 2, 4 and 7 post-LAIV. Trends in viral shedding were analyzed using a random effects logistic regression model to determine the effect of demographic variables on PCR-detected viral shedding.

RESULTS: Using viral culture, 5 participants (all with CF) shed influenza A virus (1 participant each on days 1, 4 and 7; 2 participants on day 2); 3 of these had received LAIV in the prior influenza season. No culture was positive for influenza B. All 5 participants with positive influenza A cultures also had PCR-detected influenza A virus shedding throughout follow-up. The number of participants shedding PCR-detected viruses was highest on day 1 post-LAIV administration for both influenza A and B viruses. Overall, 40 (70\%) participants with CF and 11 (58\%) healthy participants shed either PCR-detected influenza A and/or B viruses throughout follow-up. Specifically, 21 participants (28\%) shed both influenza A and B at least once, 7 (9\%) shed only influenza A virus at least once, 23 (30\%) shed only influenza B virus at least once, and 25 (33\%) did not shed any virus. Finally, there were no statistically significant differences in the odds of PCR-detected viral shedding by sex, CF status and LAIV vaccination in the previous year $(\mathrm{P}>0.05)$. However, the odds of shedding either PCR-detected influenza $\mathrm{A}$ and/or $\mathrm{B}$ virus decreased as age increased (OR 0.87, 95\% CI 0.81-0.94, $\mathrm{P}=0.001$ ).

CONCLUSION: Shedding of live vaccine-strain LAIV viruses does not appear to be frequent in children with CF. Shedding of PCR-detected LAIV viruses was most frequent the day after vaccine administration. The odds of shedding PCR-detected influenza A and/or B viruses are lower in older compared to younger children.

\section{F04}

\section{THE APPLICATION OF SEROLOGICAL AND MOLECULAR DIAGNOSTIC PROCEDURES TO IDENTIFY TRAVEL-RELATED CHIKUNGUNYA CASES IN CANADA 2014}

K Dimitrova, K Holloway, K Makowski, M Andonova, R Lindsay, $\underline{\text { M Drebot }}$

National Microbiology Laboratory, Public Health Agency of Canada, Winnipeg, MB

OBJECTIVES: Since the spring of 2014, a large increase in travel-related chikungunya cases have been diagnosed in Canada. The objective of this study was to apply various diagnostic procedures and testing algorithms for identifying imported cases of chikungunya.

METHODOLOGY: Suspect case samples from PHLs across Canada were screened using a CDC based IgM ELISA. Specimens positive for IgM were confirmed for presence of viral specific antibody using a plaque reduction neutralization test. Serum samples were also tested by real time and conventional RT-PCR for the presence of viral RNA. Further validation of diagnostic procedures were carried out using standards supplied by the FDA and the European Reference Network and incorporating additional assays such as HI and IFA platforms to confirm cases.

RESULTS: Utilizing a diagnostic algorithm employing both serological and molecular approaches, over 300 confirmed cases of chikungunya were identified in 2014. This marked a 10 to 200 fold increase in the average yearly number of chikungunya cases previously observed in Canadian travellers. Most cases with travel histories were reported from travellers to the Caribbean where the virus recently emerged; however, significant numbers of cases were also identified in travellers to the Asia - Pacific region where significant outbreaks are also occurring. Based on PCR results, a third of cases were viremic thus raising the possibility that local transmission could occur if the primary mosquito vectors become established in Canada.

CONCLUSIONS: Given the recent expansion of chikungunya throughout the Americas and on-going activity in parts of Asia, a heightened awareness of this pathogen among clinicians and the public is key. Steps are underway to transfer front-line serological testing capacity to some of PHLs to facilitate prompt diagnostic testing of suspect cases. Continued communications regarding preventative measures to decrease risk of exposure to infected mosquitoes when travelling to endemic areas is essential. 


\section{F05}

\section{DETECTION OF ENTEROVIRUS D68 IN CANADIAN LABORATORIES}

T Hatchette $^{1,2}$, SJ Drews ${ }^{3,4}$, E Grudeski ${ }^{5}$, T Booth $^{5}$, C Martineau ${ }^{6}$, K Dust $^{7}$, R Garceau ${ }^{8}$, JB Gubbay 9,10 , T Karnauchow ${ }^{11,12}$, M Krajden ${ }^{13}$, PN Levett ${ }^{14}$, T Mazzulli ${ }^{15,16}$, RR McDonald ${ }^{14}$, A McNabb ${ }^{13}$, S Mubareka ${ }^{17}$, R Needle ${ }^{18}$, A Petrich ${ }^{19}$, S Richardson ${ }^{19}$, C Rutherford ${ }^{20}$, M Smieja ${ }^{20}$, R Tellier ${ }^{21}$, G Tipples ${ }^{3,22}$, L LeBlanc ${ }^{1,2}$ ${ }^{1}$ Division of Microbiology, Department of Pathology and Laboratory Medicine, Capital District Health Authority, and Department of Pathology; ${ }^{2}$ Dalhousie University, Halifax, NS; ${ }^{3}$ Provincial Laboratory for Public Health, and Department of Laboratory Medicine and Pathology; ${ }^{4}$ University of Alberta, Edmonton, AB; ${ }^{5}$ Enterovirus and Enteric Virus Laboratory, National Microbiology Laboratory, Winnipeg, MB; ${ }^{6}$ Laboratoire de Santé Publique du Québec/Institut National de Santé Publique du Québec, Québec, QC; ${ }^{7}$ Cadham Provincial Laboratory, Winnipeg, $\mathrm{MB} ;{ }^{8}$ Centre Hospitalier Universitaire Dr George L Dumont, Moncton, NB; ${ }^{9}$ Public Health Ontario; ${ }^{10}$ University of Toronto, Toronto; ${ }^{11}$ EORLA Regional Virology Laboratory, Children's Hospital of Eastern Ontario; ${ }^{12}$ University of Ottawa, Ottawa, ON; ${ }^{13}$ Public Health Microbiology and Reference Laboratory, British Columbia Centre for Disease Control, Vancouver, BC; ${ }^{14}$ Saskatchewan Disease Control Lab, Ministry of Health, Regina, SK; ${ }^{15}$ Department of Microbiology, Mount Sinai Hospital and University Health Network; ${ }^{16}$ Department of Laboratory Medicine and Pathobiology, University of Toronto; ${ }^{17}$ Sunnybrook Health Sciences Centre, Toronto, ON; ${ }^{18}$ Public Health Laboratory \& Microbiology, St John's, NL; ${ }^{19}$ The Hospital for Sick Children, Toronto; ${ }^{20}$ St Joseph's Healthcare, Hamilton, ON; ${ }^{21}$ University of Calgary, Calgary; ${ }^{22}$ Medical Microbiology and Immunology, University of Alberta, Edmonton, AB

BACKGROUND: In late summer and early fall of 2014, widespread enterovirus type D68 (EV-D68) activity was described across North America with severe cases described in the US. Of particular concern, EV-D68 infection has been associated with acute flaccid paralysis, and fatal cases have been documented. While nucleic acid amplification tests (NAATs) have become the method of choice for the detection of respiratory viruses like enteroviruses, there is evidence that assays vary in their performance characteristics, including analytical sensitivity. This study compared the analytical sensitivity of laboratory-developed tests (LDT) and commercially available NAATs used in hospital-based and provincial public health laboratories across Canada for the detection of EV-D68.

METHODS: The lower limit of detection (LoD) of each method was determined by testing 10 -fold serial dilutions of RNA extracted from cultured EV-D68. Five replicates of each RNA dilution was shipped to participating sites. The estimated LoD for each assay was defined by Probit analysis using a probability of $95 \%$ and values were expressed as number of target copies $/ \mathrm{mL}$.

RESULTS: Our data demonstrated considerable variability in performance characteristics of assays used to detect EVD68 across Canada. Of particular interest, the Seegene RV15 and RV16 assays failed to detect EV-D68.

CONCLUSIONS: Coordinated surveillance and detection algorithms are key in the understanding of the scope of spread and spectrum of EV-D68 disease. As with any emerging pathogen, it is important to understand the limitations of each molecular assay for the detection of EV-D68 and the comparability of assays offered by reference testing services. This study provides the first report comparing the analytical sensitivity of LDTs and commercial NAATs used in Canadian laboratories for the detection of EV-D68.

\section{F06}

EVALUATION AND COMPARISON OF THE LUMINEX® XTAG RVP FAST2, SEEGENE® ANYPLEX II RV16 AND SEEPLEX RV15 VIRAL RESPIRATORY PANEL ASSAYS

S Radko, JI Stuart, G Zahariadis

Western University, London, $\mathrm{ON}$

OBJECTIVE: Timely identification of respiratory virus infection amongst patients is essential to mitigate inappropriate antibiotic use, initiate appropriate anti-viral prophylaxis or treatment and implement infection control procedures to limit transmission. As such, multiplexed molecular assays have become standard practice in most clinical virology laboratories. The goal of this study was to evaluate and compare the Seegene ${ }^{\circledR}$ Seeplex RV15 detection kit (done on Caliper Life Sciences gel based detection platform) with two next generation assays, the Seegene ${ }^{\circledR}$ Anyplex II RV16 (done on BioRad CFX-96 Real Time PCR platform) and the Luminex ${ }^{\circledR}$ xTAG RVP fast2 (done on MagPix platform) respiratory virus panels. METHODS: One Hundred and sixty-seven retrospective and 36 prospective respiratory samples were tested on all three assays. Samples were deemed to be positive if they tested positive for a virus by $2 / 3$ assays. Negative samples had to test negative by $2 / 3$ assays, while inconclusive samples were defined as those with band signal intensity between $0-100$ on the RV15.

RESULTS: There was no statistically significant difference in overall sensitivity and specificity (Table 1). Discordant rates were $47 \%$ and $21 \%$ for positive and negative samples, respectively.

CONCLUSION: Overall sensitivity and specificity of all 3 assays were similar though higher than expected discordant rates may reflect primer or chemistry differences amongst the three multiplex assays. Hence, factors such as turn-around time, the need to repeat testing and price become important variables for consideration in the decision making process for implementing such assays.

\section{TABLE 1}

\begin{tabular}{lcccccc}
\hline $\begin{array}{l}\text { Assay Sensitivity and Specificity for RV15, xTAG and RV16 } \\
\text { by Virus Type }\end{array}$ & \multicolumn{7}{c}{ RV15 } & \multicolumn{2}{c}{ xTAG } & \multicolumn{2}{c}{ RV16 } \\
\hline Viral Target & Sens & Spec & Sens & Spec & Sens & Spec \\
& $\%$ & $\%$ & $\%$ & $\%$ & $\%$ & $\%$ \\
& 93 & 95 & 88 & 100 & 92 & 98 \\
Rhino/ Enterovirus & 83 & 100 & 88 & 100 & 92 & 99 \\
RSV A/ B & 83 & 100 & 95 & 100 & 95 & 100 \\
Influenza A & 85 & 100 & 64 & 99 & 92 & 100 \\
Influenza B & 92 & 100 & 94 & 99 & 90 & 100 \\
Parainfluenzavirus & & & & & & \\
1-4 & 82 & 100 & 88 & 100 & 100 & 99 \\
Metapneumovirus & 88 & 100 & 92 & 100 & 96 & 100 \\
Coronavirus & 100 & 100 & 100 & 99 & 70 & 100 \\
Adenovirus & 50 & 99 & 44 & 100 & 63 & 100 \\
Bocavirus & 84 & 99 & 84 & 100 & 87 & 100 \\
Average & &
\end{tabular}

* Sens= Sensitivity; Spec= Specificity

\section{6:00-17:30 Oral Presentations: Session G} Room: Brown, Delta Prince Edward

\section{G01}

COMPARATIVE EVALUATION OF TWO PHENOTYPIC TESTS FOR DETECTION OF PENICILLIN BINDING PROTEIN 2A (PBP2A) IN DIVERSE RETROSPECTIVE CLINICAL STAPHYLOCOCCUS AUREUS (SA) ISOLATES

BM Willey ${ }^{1}$, P Rahman $^{1}$, O Leung ${ }^{2}$, S Dunna ${ }^{3}$, DN Grohn ${ }^{4}$, X Trimi ${ }^{3}$, P Lo $^{1,3}$, T Mazzulli ${ }^{1,3}$, SM Poutanen ${ }^{1,3}$

${ }^{1}$ Mount Sinai Hospital/University Health Network; ${ }^{2}$ University of Western Ontario, London; ${ }^{3}$ University of Toronto; ${ }^{4}$ Michener Institute, Toronto, ON

OBJECTIVES: PBP2a detection is used to distinguish SA resistant to methicillin (MRSA) from those that are susceptible (MSSA). The Denka Seiken PBP2a monoclonal agglutination has been the most commonly used test in clinical laboratories. But as it does not detect the emergent mecC-protein, and as it is laborious, other quicker tests are now under 
consideration. This study compared the Denka to the Alere PBP2a Colony Culture Test (CCT) a line assay that claims to detect mecC MRSA. METHODS: 156 highly-characterized SA including 126 genotypically distinct MRSA (116 mecA, $10 \mathrm{mecC}$ ) and 30 unrelated MSSA (13 with SCCmec remnants) were randomized to $5 \%$ sheep blood agar (BA) and Chromogenic Staphylococcus/Denim Blue agars (CA) for blinded parallel testing by Denka and CCT. As specified by Alere for CCT inoculation, 3 SA colonies were touched but not picked, and even faint blue lines were positive (pos). CCT discrepancies were retested by picking 3 colonies in parallel to 3 touched colonies.

RESULTS: Of 79 SA tested from BA, CCT was pos in 61/61 mecA (18 weak-pos) but 0/4 mecC MRSA, and negative (neg) in 14/14 MSSA, while Denka was pos in 61/61 mecA and 1/4 mecC MRSA, and neg in 13/14 MSSA (1 weak-pos). Of 77 SA tested from CA, CCT was pos in 44/55 mecA (22 weak-pos) but 0/6 mecC MRSA, and neg in 15/16 MSSA ( 1 weak-pos remnant), while Denka was pos in 55/55 mecA and 0/6 mecC MRSA, and neg in 13/16 MSSA (3 weak-pos remnants). Overall \% sensitivities (Sn)/specificities $(\mathrm{Sp})$ from BA and CA combined for CCT and Denka, respectively, were 83 (95\% CI: 76-89)/97 (95\% CI: 82->99.9) and 93 (95\% CI: 87-96)/87 (95\% CI: 70-95). 29 SA from failed tests and 19 from non-failed tests (as controls) were repeated. Using CCT data from 3 picked colony repeats, revised $\% \mathrm{Sn} / \mathrm{Sp}$ for CCT/Denka, respectively, were 91 (95\% CI: 85-95)/100 (95\% CI: 86-100) and 92 (95\% CI: 86-96)/100 (95\% CI: 86-100).

CONCLUSIONS: Denka detected all mecA MRSA from BA and CA, and CCT detected all mecA from BA but only $80 \%$ from CA $(P=0.0008)$ using manufacturers' protocols. A modified protocol using 3 picked instead of touched colonies increased the overall CCT Sn for detecting mecA MRSA from $90.5 \%$ to $99.1 \%(\mathrm{P}=0.0052)$. Denka detected (as weak-pos) 1 of 4 mecC tested on BA; but otherwise Denka and CCT missed all mecC MRSA.

\section{G02}

\section{COMPARISON OF TWO BROTH ENRICHMENT AND TWO AGARS FOR THE DETECTION OF GROUP B STREPTOCOCCUS (GBS) FROM VAGINAL-RECTAL SCREENING SPECIMENS}

P Lagacé-Wiens $^{1,2}$, B Hosseini ${ }^{2}$, G Poliquin ${ }^{2}$, S Edagiz ${ }^{2}$, JA Karlowsky ${ }^{1,2}$ ${ }^{1}$ Diagnostic Services Manitoba; ${ }^{2}$ University of Manitoba, Winnipeg, MB

BACKGROUND: GBS infection can cause both early- and late-onset neonatal disease. Widespread adoption of GBS screening and intrapartum prophylaxis has reduced the incidence of early-onset disease significantly. Further optimization of screening may result in additional benefits. As a result, there has been renewed interest to enhance GBS recovery, using new broths, new agar plates, chromogenic media as well as molecular detection. The purpose of this study was to assess the sensitivity and specificity of novel culture methods to enhance the recovery of GBS.

METHODS: Vaginal-rectal swabs were collected in duplicate from women attending routine pre-natal appointments between 34 and 36 weeks gestation. Swabs were placed in either Todd-Hewitt (TH) broth (Oxoid ${ }^{\mathrm{TM}}$ ) or RambaQuick (RQ) broth (CHROMagar ${ }^{\mathrm{TM}}$ ), which is designed to reduce overgrowth of enterococci isolated in vaginal-rectal samples. Broths were incubated overnight at $35^{\circ} \mathrm{C}$. Broths were subcultured to sheep blood agar (SBA) and Colorex ${ }^{\mathrm{TM}}$ StrepB (CSB, CHROMagar ${ }^{\mathrm{TM}}$ ). GBS were identified using colony morphology and Lancefield typing from SBA and by chromogenic reaction and Lancefield typing from CSB.

RESULTS: 45 of 204 samples were positive for GBS. There was no significant difference between testing methods. Sensitivity was $97.8 \%$ for $\mathrm{TH}$ broth subculture to SBA, and $97.8 \%$ for TH broth subculture to CSB. Sensitivity was 95\% for RQ subcultured to SBA and 97.7\% for RQ subculture to CSB. Specificity was $100 \%$ for all media. CSB allowed easy visual discrimination between Enterococcus sp. and GBS. RQ broth provided shortterm suppression of the growth of enterococci while allowing growth of GBS, but this was only observed with shorter incubation periods (i.e. $<12$ hours). CONCLUSION: Both broths were equally sensitive for the recovery of GBS. The RQ broth inhibited enterococci, but inhibition was not sustained over the typical incubation time. SBA and CSB media are equally sensitive for the identification of GBS, but CSB required fewer additional investigations as enterococci were readily recognized from non- or minimally-haemolytic GBS.

\section{G03 \\ DETECTION OF GROUP A STREPTOCOCCI (GAS) BY LOOP-MEDIATED ISOTHERMAL AMPLIFICATION (LAMP) DIRECTLY FROM SPECIMENS: A RAPID, SIMPLE AND COST-EFFECTIVE ALTERNATIVE TO CULTURE}

P layaratne ${ }^{1,2,3}, C$ Rutherford ${ }^{1,3}$

${ }^{1}$ St Joseph's Healthcare; ${ }^{2}$ McMaster University, ${ }^{3}$ Hamilton Health Sciences, Hamilton, ON

OBJECTIVES: Throat swab culture for GAS can take 24 to 48 hours and requires an anaerobic environment. Colonies of GAS may be missed if plates are overgrown with normal oral flora. The objective of this study was to develop an alternative method that is comparable in performance to culture but faster and cheaper. We evaluated the performance of a LAMP assay to detect GAS in throat swabs in E-swab medium and compared it to culture.

METHOD: Randomly selected E-swab throat specimens ( $\mathrm{n}=212)$ sent for GAS culture at Hamilton Health science were used. For the LAMP assay E-swab fluid was concentrated and added to a lysis solution prior to boiling for 15 min to obtain a crude extract of nucleic acids. For LAMP a 214bp fragment of the dnase $B$ gene was amplified using primers designed by Primer Explorer V4 software (Eiken Chemical Co). LAMP was carried out at $65^{\circ} \mathrm{C}$ for 45 min using a standard reaction mixture containing Bst 2.0 WS DNA polymerase (New England BioLabs). LAMP amplification was detected using a Genie ${ }^{\circledR}$ II (OptiGene, UK). For culture 30 $\mathrm{\mu l}$ of E-swab fluid was inoculated onto a 5\% sheep blood agar plate and incubated anerobically for 24 hours at $35^{\circ} \mathrm{C}$. Plates were examined and beta haemolytic colonies were identified using gram stain, catalase, and PathoDx latex grouping. Discordant results were examined by repeating the culture from the original specimen, transferring the swab to new e-swab medium, extracting and culturing the new specimen and repeating the LAMP on the new and original specimens. RESULTS: Forty specimens were positive for GAS by LAMP and culture. There were 13 discordant results of which 3 were culture positive and LAMP negative and 10 were LAMP positive and culture negative. The performance characteristics of LAMP as compared to culture were as follows: 93\% sensitivity, $94.1 \%$ specificity, $80 \%$ PPV and 98.1\% NPV. Post discordant analysis the sensitivity, specificity, PPV and NPV were $100.0 \%$, $96.4 \%, 88.0 \%$ and $100.0 \%$ respectively. The turn-around-time for LAMP was $90 \mathrm{~min}$ as compared to minimum 24 hours for culture. The cost for LAMP is about $\mathrm{Cdn} \$ 3.00$ per test.

CONCLUSION: Detection of GAS by LAMP is faster and more costeffective than culture with comparable performance. This assay provides promising results for rapid detection of GAS compared to culture.

\section{G04}

COMPREHENSIVE EVALUATION OF CEPHEID'S XPERT NASAL COMPLETE G3 PCR (XNC) ON THEIR GENEXPERT FOR DISTINGUISHING METHICILLIN-RESISTANT (MR) FROM METHICILLIN-SUSCEPTIBLE (MS) STAPHYLOCOCCUS AUREUS (SA) AND COAGULASE-NEGATIVE STAPHYLOCOCCI (CNS) FROM ISOLATES

BM Willey ${ }^{1}$, O Leung ${ }^{2}$, P Rahman ${ }^{1}$, X Trimi ${ }^{3}$, DN Grohn ${ }^{4}$, S Dunna ${ }^{3}$, P Lo ${ }^{1}$, T Mazzulli ${ }^{1,3}$, SM Poutanen ${ }^{1,3}$

${ }^{1}$ Mount Sinai Hospital/University Health Network, Toronto;

${ }^{2}$ University of Western Ontario, London; ${ }^{3}$ University of Toronto; ${ }^{4}$ Michener Institute, Toronto, ON

OBJECTIVES: Rapid PCR detection of MRSA directly from clinical specimens is problematic due to 1) genetic similarities between mecA in SA and CNS, 2) prevalence of MSSA with remnant (rem-SA) Staphylococcus Cassette Chromosome-mec (SCCmec) sequences that no longer carry mecA, and 3) continued evolution of SCCmec and mec determinants. PCR assays are notoriously prone to false-positives (FP) due to 1) and 2) and false-negatives (FN) due to 3). We evaluated the XNC with the aim to speed up institution of appropriate therapy in cases of short-incubation (<6h) blood culture SA identified by MALDI-TOF. 
METHODS: 181 highly-characterized genotypically-unique clinical isolates studied included 126 MRSA (116 mecA, 10 mecC), 30 MSSA (13/30 rem-SA) and $25 \mathrm{CNS}$. On recovery from $-80^{\circ} \mathrm{C}$, strains were plated twice: $50 \% / 50 \%$ to $5 \%$ sheep blood/Chromogenic Staph or Demin Blue agars (Oxoid). Bacterial suspensions prepared in saline equivalent to $\sim 0.1 \mathrm{MacFarland}$ STD were inoculated by swab to XNC buffers, after which testing was as per Cepheid. XNC results including crossing thresholds (Ct) for SPA (detects SA), mec (detects mecA) and SCC (detects SCCmec) were correlated with strain characteristics. RESULTS: The XNC SPA target correctly identified 156/156 SA (Ct range: $16.7-25.7 ; 100 \% ; 95 \%$ CI: $97-100$ ) and excluded SA (and MRSA) in 25/25 CNS (Ct: 0; 100\%; 95\% CI: 84-100) regardless of agar used. Mec and SCC were amplified in 116/116 mecA MRSA (100\%; 95\% CI: 96.2-100) with $\mathrm{Ct}$ ranges 16.7-26 and 17.6-32.7, respectively. Not surprisingly, these targets were detected in 0/10 mecC MRSA leading to an overall MRSA detection sensitivity (Sn) of 92.1\% (95\% CI: 85.9-95.8). Although 0/30 MSSA was reported as MRSA (100\% specificity; $95 \%$ CI: 86.5-100), amplification occurred for SCC alone in 11/13 rem-SA (Ct: 19.6-22.8) and 3/17 SA with no remnants (Ct: 27.2-37.6), for mec alone in 1 rem-SA (Ct: 37.9), and SCC and mec were both amplified in 1/17 MSSA (Ct: 36.4, 36.7).

CONCLUSIONS: With the exception of mecC MRSA, this retrospective study data supports the use of the XNC assay directly from purified clinical isolates. However, prior to its clinical implementation for testing short incubation blood culture SA, additional prospective evaluations should be undertaken to ensure 1) all currently circulating MRSA are indeed detected, and 2) that mixed MSSA/MR-CNS cultures do not lead to false-reports of MRSA.

\section{G05}

DIRECT REPORTING CEFAZOLIN FROM VITEK 2 AST N208 FOR E. COLI, K. PNEUMONIAE AND P. MIRABILIS ISOLATED FROM URINE CULTURES USING 2014 CLSI INTERPRETATION

IXiong1', B Langford 2', H Kong1', D Hancock'1, S Krajden ${ }^{1,3}$, M Downing ${ }^{2}$, W Chapman ${ }^{1,3}$

${ }^{1}$ Department of Laboratory Medicine, St Joseph's Health Center; ${ }^{2}$ Antimicrobial Stewarship, St Joseph's Health Center; ${ }^{3}$ University of Toronto, Toronto, ON

BACKGROUND: There have been amendments in cefazolin interpretations through the years (see Table). During 2010 - 3, our laboratory performed a Kirby-Bauer (K-B) confirmation test for cefazolin before reporting Vitek 2 AST results for E. coli, K. pneumoniae and P. mirabilis isolated from urinary specimens to accommodate the lowered breakpoints.

\begin{tabular}{|lccc|}
\hline Cefazolin interpretation & $\begin{array}{c}\text { Susceptible } \\
\text { (UTIs, mg/L) }\end{array}$ & Intermediate & Resistant \\
2014 CLSI M100-S24 & $<=16$ & (I) & (R) \\
\end{tabular}

This study is to verify if the lab can report cefazolin directly from Vitek 2 testing without an extra K-B test based on the new 2014 breakpoints.

METHODS: Cefazolin susceptibilities of 129 urinary clinical isolates of E. coli, K. pneumonia and P. mirabilis in 2013 were compared, between their Vitek 2 and K-B (reference method) testing results, using the new UTI 2014 CLSI interpretations for cefazolin ( $30 \mu \mathrm{g} / \mathrm{disc}) \mathrm{S}: \geq 15 \mathrm{~mm}$ and $\mathrm{R}: \leq 14 \mathrm{~mm}$. Category agreement was measured and described as minor, major and very major errors.

RESULTS: Based on the 2014 new K-B interpretation, direct reporting cefazolin from Vitek 2 AST against E. coli, K. pneumonia, and P. mirabilis gave a category agreement of $94 \%$.

\section{TABLE 1}

Category Agreement between K-B vs Vitek 2 for Cefazolin of 129 isolates

\begin{tabular}{llll}
\hline Error Type & K-B Method & Vitek 2 Method & No. of Errors (\%) \\
\hline Minor Errors & S or I & I or S & 0 \\
& R or I & I or R & 0 \\
Major & S & R & $4(3 \%)$ \\
Very Major & R & S & $4(3 \%)$ \\
\hline
\end{tabular}

CONCLUSION: It is generally acceptable to report cefazolin directly from Vitek 2 for the three species of Enterobacteriaceae isolated from urine cultures using the new 2014 CLSI interpretation. It saves cost $>\$ 3000 / y r$.

*Vitek 2 interpretation based on (FDA Cleared Device).

\section{G06}

RAPID DIAGNOSTIC TESTING AND LACTOBACILLUS REUTERI THERAPY FOR CHILDREN WITH SEVERE ACUTE GASTROENTERITIS IN BOTSWANA: A PILOT, FACTORIAL, RANDOMIZED, CONTROLLED, CLINICAL TRIAL

IM Pernica ${ }^{1}$, AP Steenhoff ${ }^{2,3}$, M Mokomane ${ }^{4}$, K Lechiile ${ }^{2}$, JB Moorad ${ }^{2}$, M Smieja ${ }^{1}$, L Mazhani ${ }^{5}$, M Loeb ${ }^{1}$, K Stordal ${ }^{6}$, DM Goldfarb ${ }^{1,2}$

${ }^{1}$ McMaster University, Hamilton, ON; ${ }^{2}$ Botswana-UPenn Partnership, Gaborone, Botswana; ${ }^{3}$ Children's Hospital of Philadelphia, Philadelphia, PA, USA; ${ }^{4}$ National Health Laboratory, Gaborone; ${ }^{5}$ University of Botswana, Gaborone, Botswana; ${ }^{6}$ Norwegian Institute of Public Health, Oslo, Norway

OBJECTIVES: To demonstrate the feasibility of an RCT designed to measure the benefit of rapid enteric diagnostic testing and probiotic therapy for children admitted to hospital in Botswana with severe acute gastroenteritis. METHODS: Children with acute diarrhoeal disease aged 2-60 months admitted to any of three Botswana hospitals were eligible to participate if they did not have bloody stools or signs of sepsis. Participants received standard fluid rehydration, zinc treatment, and were randomized to one of four arms: 1) rapid diagnostic testing (plus targeted antimicrobial therapy if indicated) plus Lactobacillus reuteri therapy $\times 60$ days; 2 ) rapid diagnostic testing (plus targeted antimicrobial therapy if indicated) plus placebo therapy $\times 60$ days; 3 ) standard care (delayed diagnostic testing) plus L. reuteri therapy $\times 60$ days; or 4 ) delayed diagnostic testing plus placebo therapy $\times 60$ days. Rapid testing consisted of multiplex PCR assays to detect Shigella, Campylobacter, enterotoxigenic E. coli LT/ST toxin, Salmonella, and Cryptosporidium. For this pilot study, achievement of feasibility outcomes was primary; these included validation of the diagnostic testing protocols, ensuring that testing and appropriate treatment could be integrated into clinical care, and verifying adequate recruitment rates. Clinical outcomes included height at 60 days, weight at 60 days, 60 -day mortality, and recurrence of diarrhoea in the 60-day followup period.

RESULTS: In a 6-month study period, 76 participants were enrolled. The median participant age was 10.8 months, $49 \%$ had been exposed to HIV in utero, and 6 had severe acute malnutrition. 34 of 37 children randomized to rapid testing had results reported within 24 hours, and 19 of 20 with a treatable pathogen detected via rapid testing had antimicrobials begun within $24 \mathrm{~h}$. Mean 60-day height was $2.56 \mathrm{~cm}$ greater in the rapid diagnostics arms compared to $2.07 \mathrm{~cm}$ greater in the standard treatment arms, and only 6 of 34 children (18\%) randomized to rapid testing developed repeated diarrhoea in the followup period, compared to 12 of 34 (35\%) in the control arms. Probiotic/placebo groups are still blinded.

CONCLUSIONS: We have demonstrated feasibility of the trial protocol. An adequately-powered multicentre RCT to precisely quantify the benefit of rapid diagnostics and L. reuteri probiotic therapy in this population is indicated. 
16:00-17:30 Oral Presentations: Session H Room: Chandler, Delta Prince Edward

\section{H01}

IS LYME DISEASE BEING MISSED IN MULTIPLE SCLEROSIS PATIENTS IN NEW BRUNSWICK?

V Lloyd ${ }^{1,2}$, P Cook ${ }^{1,3}$, T Hatchette ${ }^{4,5}$, R Lindsay ${ }^{6}$, D Webster ${ }^{1,7}$, G MacLean 1,3

${ }^{1}$ St John Regional Hospital, Saint John; ${ }^{2}$ Mount Allison University, Sackville; ${ }^{3}$ Horizon Health, Saint John, NB; ${ }^{4}$ Capital District Health Authority; ${ }^{5}$ Dalhousie University, Halifax, NS; ${ }^{6}$ Zoonotic Diseases and Special Pathogens, National Microbiology Laboratory, Public Health Agency of Canada, Winnipeg, MB; ${ }^{7}$ Dalhousie Medicine New Brunswick, Saint John, NB

INTRODUCTION: Lyme disease is an emerging infection in Atlantic Canada. The concern has been raised that late Lyme disease is being misdiagnosed as other chronic diseases such as multiple sclerosis (MS). We tested a cohort of MS patients in New Brunswick to determine whether any patients within this cohort had laboratory test results consistent with Lyme disease.

METHODS: 90 patients attending the MS clinic in Saint John, New Brunswick were tested for the presence of antibodies for Borrelia burgdorferi. Serological testing was performed using the two-step algorithm in accordance with the IDSA guidelines. Each patient's serum was first tested with the C6 B. burgdorferi (Lyme) ELISA (Immunetics, Inc., Boston, Massachusetts, USA). Samples that produced positive or equivocal results on the C6 ELISA were subsequently tested for IgG antibodies using a Western blot (B. burgdorferi US (IgG), Euroimmun, Luebeck, Germany). A positive Western blot required the presence of 5 of 10 significant bands. RESULTS: Although, 6 of 90 (6.7\%) MS patients had serum that was reactive on the C6 EIA, none of these reactive EIA specimens could be confirmed using the Western blot.

CONCLUSION: The seroprevalence of antibodies against B. burgdorferi in this cohort of NB MS patients was zero by the IDSA two-step algorithm.

\section{H02}

\section{PREVALENCE AND EPIDEMIOLOGY OF METHICILLIN- RESISTANT STAPHYLOCOCCUS AUREUS COLONIZATION IN AN ABORIGINAL COMMUNITY}

P Daley ${ }^{1}$, J Bajgai ${ }^{1}$, C Penney ${ }^{1}$, H Whitney ${ }^{1}$, G Golding ${ }^{2}$, D Allison ${ }^{1}$

${ }^{1}$ Memorial University, St John's, NL; ${ }^{2}$ National Microbiology

Laboratory, Winnipeg, MB

BACKGROUND: Canadian Aboriginal populations are at an increased risk for community-acquired MRSA infection for unknown reasons. OBJECTIVES: To describe the prevalence and molecular epidemiology of MRSA colonization among humans and animals in an Aboriginal community.

METHODS: Adult and child volunteers were sought from clinic visits, house visits, school and work locations, between October 6 and November 27, 2014. A single nasal swab was collected. Dogs undergoing spay/neuter surgery were swabbed in nasal, oral, anal, groin and wound sites. Univariate regression (SPSS 22.0) and spa typing were performed.

RESULTS: 461 residents were approached, 13 refused consent, 4 were missing data, leaving 444 eligible participants. 242/444 (54.5\%) were women, mean age was $25.7 \mathrm{yrs}$ (SD 19.8), 169/444 (38.1\%) had received antimicrobials in the last 12 months. MRSA prevalence was $111 / 444$ (25.0\%). Significant predictors of MRSA colonization included number of rooms in the house $(\mathrm{RR}=0.73, \mathrm{P}=0.01)$, antibiotic use in the last 12 months $(\mathrm{RR}=1.18, \mathrm{P}=0.04)$, owning a $\operatorname{dog}(\mathrm{RR}=0.11, \mathrm{P}<0.01)$, feeding a dog regularly $(R R=16.14, P<0.01)$, and using a dog for recreation $(R R=0.022$, $\mathrm{P}=0.01$ ). Age, gender, ethnicity, number of people in house, number of sinks in house, admission to hospital, surgery, indwelling medical device, dialysis, prior MRSA infection, history of skin infection, MRSA among contacts, intravenous drug abuse, new tattoos or piercing, chronic skin condition, exposure to healthcare or corrections or homeless shelter or daycare or veterinary care did not predict MRSA colonization. 89/111 (80.1\%) of human staphylococci were CMRSA10, 82/89 (92.1\%) of which were Spa type t008. 55/157 (35.0\%) of dogs were colonized with coagulasepositive staphylococci.

CONCLUSIONS: MRSA colonization is not associated with healthcare contact, hygiene factors, skin disease or contact with MRSA, but may be associated with crowding, antibiotic use and dog exposure. Human MRSA is mostly clonal. Further genotyping may demonstrate human-animal inter-transmission.

\section{H03}

\section{EVALUATION OF THREE CHROMOGENIC MEDIA FOR SCREENING SURVEILLANCE SPECIMENS FOR THIRD GENERATION CEPHALOSPORIN RESISTANT ENTEROBACTERIACEAE}

C Rutherford ${ }^{1}$, M Partovi ${ }^{1}$, L Monkman ${ }^{1}$, D Yamamura ${ }^{1,2}$

${ }^{1}$ Hamilton Regional Lab Medicine Program; ${ }^{2}$ McMaster University Department of Pathology and Molecular Medicine, Hamilton, ON OBJECTIVE: This study evaluated three media for the detection of Enterobacteriaceae that posses genes for extended spectrum beta lactamases, ampC or carbapenemases.

MATERIALS AND METHODS: Chromogenic bi-plates of Colorex ${ }^{\mathrm{TM}}$ $\mathrm{KPC}$ and Colorex ${ }^{\mathrm{TM}}$ C3GR (C3GR) were obtained from Alere Canada. chromID ${ }^{\mathrm{TM}}$ CARBA (CIC) plates were obtained from bioMérieux Canada. Rectal swabs from 339 patients were vortexed in sterile saline and $50 \mu \mathrm{L}$ was applied to each medium. A challenge set of known multi-resistant Enterobacteriaceae $(n=31)$ was also tested. Plates were incubated as recommended and examined for degree of growth and colony colour. Isolates were identified using Vitek ${ }^{\circledR}$ MS (bioMérieux) MALDI-TOF. Resistance mechanisms were elucidated using the CLSI ESBL disk test, Neo-Sensitabs ${ }^{\mathrm{TM}}$ Carbapenemase Confirmation tablets (Rosco Diagnostica) and PCR.

RESULTS: Both CRE specific agars were very selective. Escherichia coli colonies were pink on all plates. Citrobacter spp. were blue with a pink halo on C3GR, blue on KPC and dark pink on CIC. Klebsiellae and Enterobacter spp. were blue, green or blue-green. From the challenge set, C3GR medium detected 29 CRE (100\%), KPC detected 23/29 (79.3\%) and CIC detected $26 / 29(89.7 \%)$. A CTX-M isolate was detected on all media and a plasmid ampC isolate was only detected on C3GR as expected. From routine specimens, C3GR detected 31 ESBL producing organisms, 1 of which grew on $\mathrm{KPC}$ agar and $10 \mathrm{AmpC}$ producers, none of which grew on the other media. Seven isolates with an ESBL and plasmid ampC grew on C3GR, 2 of which grew on KPC and 1 on CIC.

CONCLUSIONS: Colour specificity for Escherichia coli was excellent on all media. Differentiation of Enterobacters from Klebsiellae was not possible and Citrobacters had various presentations.

Colorex $^{\mathrm{TM}}$ C3GR agar provides the greatest yield of multi-resistant Enterobacteriaceae. Combining C3GR agar with Colorex ${ }^{\mathrm{TM}} \mathrm{KPC}$ and MALDI-TOF identification provides an efficient and cost effective solution for nosocomial screening.

\section{H04}

COMPARISON OF THE NEW MRSASELECT II, BRILLIANCE MRSA 2 MEDIA WITH CURRENT VERSIONS OF MRSASELECT AND DENIM BLUE FOR THE DETECTION OF MRSA

\section{P Kornherr, H Faizullah, E Pickles, M Sohrabi}

Gamma-Dynacare Medical Laboratories, Ottawa, ON

OBJECTIVES: Two commercial media manufacturers have recently modified their respective formulations of chromogenic MRSA plates. A limited run of 500 of Thermo Fisher Oxoid's Brilliance MRSA 2 (DB2) and BioRad's MRSASelect II (MS2) were available for evaluation. Each claim to offer more robust growth at reduced incubation times of $18 \mathrm{hrs}$. To assess, we compared their previous versions MRSASelect (MS) and Denim Blue (DB) with new versions of each at various incubation times in 3 phases 1) retrospectively: 100 characterized MRSA strains split at dilutions of $10^{3}$, $10^{2}$ and $10 \mathrm{CFU} /$ plate 2) 350 prospective nasal, rectal/ perineal, wound swabs and 3) 50 recent retrospective low colony count positive swabs. METHODS: 1) 100 known positive MRSA were freshly subcultured and 
split into thirds diluted to each of approximately $10^{3}, 10^{2}$ and 10-100 CFU/ plate. General colony size, count and clarity was compared at 12, 16, $18 \mathrm{hrs}$ incubation 2) 350 new incoming samples were set up on each of 4 plates in random order and growth/no growth/breakthrough growth assessed at 12, 16, $18 \mathrm{hrs}$ and 3) 50 plates of each reset up with patient swabs of known low CFU (+/- growth) read at 18 and 24 hrs.

RESULTS: 1 ) Overall Sensitivity at 12 hrs: 61\% MS; 85\% DB; 88\%DB2 and $90 \% \mathrm{MS} 2$. At $18 \mathrm{hrs}$ all $100 \%$. Growth ratio overall at $18 \mathrm{hrs} \mathrm{(+/-}$ $: 1+: 2+$ as \%) : MS 81:19:0; DB 45:45:10; DB2 33:45:22; MS2 16:48:362) 350 samples identified 34 positives ( $10 \%$ positivity rate) through at least one positive plate. Sensitivity at $18 \mathrm{hrs:}$ MS:92\%; DB: $94 \%$; MS2 and DB2:97\%. Specificity at 18 hrs: MS2: 92\%; DB2: 96\%; MS and DB: $99 \%$. 3) Detectability of low CFU DB2 $>$ MS2 $>$ DB $>$ MS. Bacillus species were the noteworthy false positive growths on DB2 and MS2. Colonial morphology helped to differentiate from true MRSA.

CONCLUSIONS: Clarity of positives: MS2 $>$ DB2 $>$ DB $>M S$; Sensitivity MS2 $>$ DB2 $>$ MS $=\mathrm{DB}$; Specificity: $\mathrm{DB}=\mathrm{MS}>\mathrm{DB} 2>\mathrm{MS} 2$; relative amount of growth $\mathrm{MS} 2>\mathrm{DB} 2>\mathrm{DB}>\mathrm{MS}$. Both new formulations are desirable over predecessor. In particular MS2 showed most improvement in sensitivity and clarity but not specificity. DB2 improvements were slight in sensitivity and clarity. Both new versions captured all positive growths within $18 \mathrm{hrs}$ and in most cases could detect MRSA presence at $12 \mathrm{hrs}$ as a possibility for pre-screening earlier.

\section{H05}

\section{A DIRECT COMPARISON OF TOXIGENIC CLOSTRIDIUM DIFFICILE BY DUAL RAPID TEST CASSETTE VERSUS TRADITIONAL ENZYME IMMUNE ASSAY / PCR ALGORITHMIC METHODS}

$\underline{\text { R Moore }}^{1}$, G German ${ }^{1,2}$

${ }^{1}$ Prince County Hospital, Summerside; ${ }^{2}$ Health PEI, Division of Microbiology, Charlottetown, PE

BACKGROUND: The Prince County Hospital, PEI microbiology laboratory receives about 27 specimens per month for C. difficile with a $15.3 \%$ positivity rate. There is only one specimen run per day to the offsite laboratory that does 5 days a week GDH antigen and three times a week PCR testing. Various delays lead to over $40 \%$ of positives taking greater than 60 hours to result. A rapid method was studied.

METHODS: All samples were evaluated using the Bristol Score for stool consistency and only those having a score of $5-7$ were tested. 100 screened samples were tested with the C. DIFF QUIK CHEK COMPLETE (a dual rapid test cassette EIA for GDH antigen and toxin $A \& B$ ) and then subsequently sent on for GDH EIA (CDIFF CHEK-60) and PCR toxin testing (BD GeneOhm Cdiff for $t c d B$ ).

RESULTS: $86 \%$ of samples tested exactly the same by all methods. Another $10 \%$ tested antigen positive, toxin negative by the rapid test and antigen positive, toxin positive by the algorithmic method. This was determined to be a direct result of the increased sensitivity of the PCR method. $4 \%$ did not show some correlation and were sent out of province for additional investigation. Cost analysis between the 2 methods was performed and ranges between a saving of $7.9 \%$ to an increase of $9.6 \%$ of non-labour costs were obtained depending on the approach to testing.

CONCLUSIONS: $98 \%$ of samples were essentially equivalent. $86 \%$ of samples tested rapidly so a significant reduction in turnaround time was evident. The one false positive Toxin EIA with a very faint line that in the future will be considered indeterminate. There was one false negative. The overall test performance using the algorithmic method as the gold standard is a sensitivity of $98.9 \%$ specificity of $88.9 \%$. The rapid testing method when added to modified algorithm is accurate and at least cost neutral.

\section{H06 \\ SUPERIORITY OF REAL-TIME PCR OVER OTHER ANTIGEN TESTING FOR SYPHILIS DETECTION}

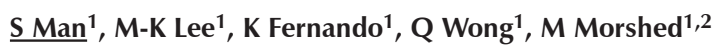
${ }^{1}$ BC Public Health Microbiology and Reference Laboratory; ${ }^{2}$ University of British Columbia, Vancouver, BC

BACKGROUND: The clinical diagnosis of syphilis is complicated due to its diverse clinical manifestations, complex testing algorithms and variable antigen detection methods. In this study, we focus on comparing our realtime PCR assay to other antigen detection methods, namely, darkfield (DF) microscopy, direct fluorescent antibody (DFA) assay, as well as a firstgeneration conventional PCR. We intend to highlight trends and infer clinical relevance as we advance our testing methodologies.

METHOD: We compared the results of DF microscopy, DFA microscopy, and a conventional PCR which targets polA gene to our real-time PCR which targets both polA and tp47 protein genes. Two by two contingency tables were used for comparative analysis.

RESULTS: DF(100\%), DFA (100\%) and conventional PCR (100\%) had comparable specificity to real-time PCR but each test had a much lower sensitivity. DF had the poorest sensitivity at $38.5 \%$, DFA at $57.6 \%$, and conventional PCR at $81.3 \%$. The increasing sensitivity trend can be attributed to the advancement of test method technology which can provide more reliable and consistent results. Real-time PCR has the advantage of detecting $T$. pallidum down to a single spirochete, whereas microscopy is reliant on sample integrity, technologist's expertise, and homogenous sample distribution on the sample slide for detection. Another advantage of the real-time PCR is that more stringent control and quality control settings can be used for added sensitivity and specificity.

CONCLUSION: Real-time PCR provided greater sensitivity and specificity than traditional microscopic and molecular methods. The semiquantitative data generated from the real-time assay can also provide a means of using statistical rules such as the Levey-Jennings method to better manage the consistency and quality of the entire analytical process.

\section{1:10-12:25 Oral Presentations: Session I Room: Henry, Delta Prince Edward}

\section{1} FULL GENOME ANALYSIS OF ENTEROVIRUS D-68 STRAINS
CIRCULATING IN ALBERTA, CANADA

K Pabbaraju' ${ }^{1}$, S Wong', SJ Drews ${ }^{2,3}$, G Tipples ${ }^{2,3}$, R Tellier ${ }^{1,4}$

${ }^{1}$ Alberta Health Services, Provincial Laboratory for Public Health (ProvLab), Calgary; ${ }^{2}$ Alberta Health Services, Provincial Laboratory for Public Health (ProvLab); ${ }^{3}$ University of Alberta, Edmonton; ${ }^{4}$ University of Calgary, Calgary, AB

INTRODUCTION: Apart from respiratory tract infections and exacerbation of asthma EV-D68 has also been implicated in several other clinical presentations. Recently acute neurologic illness with focal limb weakness in children and acute flaccid paralysis with anterior myelitis has been linked to EV-D68.

METHODS: Four different EV-D68 positive samples from patients in Alberta, Canada were used to determine near full-length genome sequences. Primers designed based on the full genome sequence of the prototype human EV-D68 Fermon strain were used to amplify overlapping fragments spanning the entire genome. Full-length genome sequences were assembled using Seqscape v3.0 and Sequencing Analysis v6.0 (ABI). Nucleic acid and protein sequence alignments were performed using Clustal W. Neighbour joining trees were constructed using the JukesCantor model and 1000 bootstrap replicates in MEGA v6.

RESULTS: The currently circulating EV-D68 strains are closely related to the contemporary strains circulating world-wide based on VP1 phylogenetic analysis, but have diverged considerably from the prototype Fermon strain. Phylogenetic analysis shows that the EV-D68 strains sequenced from all four patients in Alberta belong to lineage 2 based on Meijer et al and variant 1.2.1 and 1.2.2 based on the classification scheme by Lauinger. Consistent with these lineage designations for our sequences, the deletion at amino acid 141 in VP1 was absent and two deletion blocks were observed in the 5'UTR. Some of the Alberta strains showed amino acids changes at 142 and 143 that could affect the tertiary structure and protein function, however, no changes were noted in the antigenic epitope regions of the BC and DE loops.

CONCLUSIONS: EV-D68 was not a commonly reported enterovirus serotype between 1970 and 2005, however since then reports in the literature have suggested a greater incidence worldwide, independent of 
increased surveillance and availability of sensitive detection methodologies. It has been suggested that mutations in the VP1 gene causing altered antigenicity or lack of immunity in the younger population due to the lack of circulation in the recent years may be responsible for the increased detection of EV-D68 worldwide.

\section{2}

GENERATION OF A CAMPYLOBACTER JEJUNI

SURVEILLANCE DATABASE IN ALBERTA UTILIZING THE SUBTYPING METHOD COMPARATIVE GENOMIC FINGERPRINTING

\section{$\underline{\mathrm{LChui}}{ }^{1,2}, \mathrm{~A} \mathrm{Ma}^{1}$}

${ }^{1}$ University of Alberta; ${ }^{2}$ Provincial Laboratory for Public Health, Edmonton, $A B$

OBJECTIVES: Campylobacter jejuni infection due to ingestion of contaminated food or water is a leading cause of gastroenteritis. Subtyping methods for C. jejuni surveillance such as pulsed-field gel electrophoresis (PFGE), which is utilized by PulseNet Canada, have been developed. However, these methods are unable to overcome the difficulties of the high genetic diversity and chromosomal rearrangements in C. jejuni. An alternative method known as comparative genomic fingerprinting (CGF) was developed in the Laboratory for Foodborne Zoonoses by the Public Health Agency of Canada in Lethbridge, Alberta. CGF was adopted to build a C. jejuni surveillance database in Alberta as PFGE was not routinely used. METHODS: A total of 304 known clinical C. jejuni isolates across Alberta health zones from 2006 to 2009 were genotyped for the presence or absence of 40 target accessory genes using capillary electrophoresis following multiplex polymerase chain reaction (PCR). Presence or absence of a gene was determined by analysis of generated electropherograms and gels by the computer software and user verification. Genomic fingerprints for each isolate comprising genotyping results were used to determine genetic relatedness amongst strains and generate clusters sharing 100\% homology. RESULTS: There was high concordance between results generated with CGF, PFGE and epidemiological data pertaining to outbreak cases. Furthermore, new clusters between sporadic isolates were discovered, suggesting identification of outbreak cases that were previously not detected due to the lack of evidence or typing method for correlation. Subtyping with CGF is simple, robust, and has a fast turnaround time without sacrificing discriminatory power.

CONCLUSIONS: CGF is an alternative subtyping method for C. jejuni surveillance. Genomic fingerprints generated by CGF showed high concordance with available PFGE results and potentially revealed case associations previously not detected. The Alberta database will continue to expand with the inclusion of additional genomic fingerprints.

\section{3}

\section{WHOLE GENOME SEQUENCING TO DIFFERENTIATE HIGHLY CLONAL SALMONELLA ENTERICA SEROTYPE HEIDELBERG ISOLATES INVOLVED IN OUTBREAKS IN THE PROVINCE OF QUEBEC}

S Bekal' ${ }^{1,2}$, G Beaudry ${ }^{1}$, E Fournier ${ }^{1}$, F Doualla-Bell ${ }^{1,3}$, A Reimer ${ }^{4}$, C Berry ${ }^{4}, \underline{\text { C Tremblay }}{ }^{1,5}$

${ }^{1}$ Laboratoire de Santé publique du Québec, Institut national de santé publique, Ste-Anne de Bellevue; ${ }^{2}$ Université de Montréal; ${ }^{3}$ McGill University, Montréal, QC; ${ }^{4}$ National Microbiology Laboratory, Winnipeg, MB; ${ }^{5}$ Centre de recherche du centre hospitalier de I'Université de Montréal, Montréal, QC

BACKGROUND: Salmonella enterica is a common cause of food borne outbreaks. Salmonella enterica serotype Heidelberg is the third most encountered serovar causing human salmonellosis in Canada and the second one in the province of Quebec. This serotype is highly clonal and over 50\% of strains isolated in Quebec exhibit the same PFGE pattern SHEXAI.0001/SHEBNI.0001, leading often to unsuccessful outbreak investigations.

OBJECTIVE: This study aimed to evaluate the core genome SNP phylogeny method for discrimination of highly clonal S. Heidelberg strains involved in different outbreaks.
METHODS: Forty-six strains involved in 3 epidemiologically documented outbreaks in Quebec (2012, 2013 and 2014) were subjected to whole genome sequencing and core genome SNP analysis using SNVPhyl pipeline at the National microbiology laboratory (NML). A custom script to filter out all repeat regions and also remove any known mobile elementssuch as phage, islands was done. These strains exhibit the same pulsed-field gel electrophoresis pattern (SHEXAI.0001/SHEBNI.0001) and associated to phage types 19 or 26. Additionally, 15 sporadic strains with different PFGE patterns including the common one were included in this study.

RESULTS: Phylogenetic tree based on core genome SNP analysis allowed to clustering outbreak strains into 3 distinct clusters with concordance with epidemiological data. The number of SNP difference within strains from the same outbreak was comprised between 0 and 2 SNP. Interestingly, strains from outbreak $1(n=11,2012)$ and outbreak $3(n=12,2014)$ exhibiting the same PFGE and phage type patterns (SHEXAI.0001/SHEBNI.0001, PT 19) showed a SNP variation of 73. Strains from outbreaks $2(n=8,2013)$ exhibited a distinct phage type (PT 26) and showed a SNP difference of 82 and 147 compared to outbreak 1 and 3, respectively.

CONCLUSIONS: This study demonstrates that despite the high clonality of this serotype, core genome SNP was able to differentiate strains belonging to the same outbreak from others. This tool offers a powerful alternative for strain typing during outbreak investigations.

\section{4}

USE OF NEISSERIA GONORRHOEAE MULTIANTIGEN SEQUENCE TYPING (NG-MAST) TO CHARACTERIZE THE EPIDEMIOLOGY OF GONORRHEA IN NOVA SCOTIA, 2010-2014

E Schleihauf ${ }^{1}$, I Martin², N Whelan ${ }^{3}$, R Taylor ${ }^{3}$, T Hatchette ${ }^{3,4}$, D Haldane ${ }^{3,4,5}$

${ }^{1}$ Public Health Agency of Canada, Ottawa, ON; ${ }^{2}$ National Microbiology Laboratory, Winnipeg, MB; ${ }^{3}$ Capital District Health Authority; ${ }^{4}$ Department of Pathology, Dalhousie University; ${ }^{5}$ Nova Scotia Provincial Public Health Laboratory, Halifax, NS

BACKGROUND: Ongoing transmission of gonorrhea is made possible by core groups of infected individuals. Understanding the characteristics of the core groups supporting gonorrhea transmission and the antimicrobial susceptibilities of the gonococcal isolates transmitted can inform public health prevention and control programs, as well as treatment guidelines. METHODS: Clinical gonococcal isolates from Halifax, Nova Scotia were submitted to the National Microbiology Laboratory for further characterization. Isolates were typed by Neisseria gonorrhoeae-Multi-Antigen Sequence Typing (NG-MAST). Sequence types were grouped if they differed by three base pairs or less in the DNA sequences of the por and tbpB genes. Antimicrobial susceptibility testing (AST) provided minimum inhibitory concentrations (MICs) for eight antibiotics for each isolate via the agar dilution method as per Clinical Laboratory Standards Institute guidelines. RESULTS: Of 398 gonorrhea positive specimens between 2010 and 2014, isolates were grown and NG-MAST and AST results were available for 151 (38\%). Isolates were assigned to 42 NG-MAST types. Six identified NG-MAST groups comprised 79\% of all isolates. Isolates collected in 2014 indicated continued transmission of some NG-MAST groups for over three years. Overall, $58 \%$ of isolates were susceptible strains and none of the isolates were resistant to cefixime or ceftriaxone. In the largest NG-MAST group ( $n=57)$, all isolates were susceptible and $39 \%$ were isolated from males. Isolates with decreased susceptibility to third generation cephalosporins belonged to one NG-MAST group ( $\mathrm{n}=8,75 \%$ male), including five isolates with decreased susceptibility to cefixime $(\mathrm{MIC}=0.25 \mathrm{mg} / \mathrm{L})$ and four with decreased susceptibility to ceftriaxone (MIC $=0.125 \mathrm{mg} / \mathrm{L}$ ).

CONCLUSIONS: The NG-MAST groups identified were associated with distinct demographics of patients and susceptibility profiles of isolated organisms depicting discrete sexual networks. Base pair changes over time result in assignment of different sequence types, therefore phylogenetic NG-MAST classification is required for elucidating local epidemiology. Incorporation of NG-MAST into routine surveillance activities could inform prevention and control programs. 


\section{5}

\section{DETECTION OF LISTERIA MONOCYTOGENES USING REAL-TIME AMPLIFICATION ASSAYS}

L Chui $^{1}{ }^{1,2}$, LM Patterson-Fortin ${ }^{1}$, S Chong ${ }^{3}$, J Mahony ${ }^{3}$, F Pagotto ${ }^{4}$, M Graham ${ }^{5}$, G Van Domselaar ${ }^{5}$, C Berry ${ }^{5}$, N Knox ${ }^{5}$, K Seyer ${ }^{6}$, J Zhang ${ }^{7}$, S Bekal ${ }^{8}$, J Chen ${ }^{9}$, N Prystajecky ${ }^{10}$, P Slade ${ }^{11}$

${ }^{1}$ University of Alberta; ${ }^{2}$ Provincial Laboratory for Public Health, Edmonton, AB; ${ }^{3}$ McMaster University, Hamilton; ${ }^{4}$ Listeriosis Reference Service, Bureau of Microbial Hazards, Health Canada, Ottawa, ON; ${ }^{5}$ National Microbiology Laboratory, Public Health Agency of Canada, Winnipeg, MB; ${ }^{6}$ Canadian Food Inspection Agency, St Hyacinthe, QC; ${ }^{7}$ Alberta Innovates Technology Futures, Vegreville, AB; ${ }^{8}$ Laboratoire de santé publique du Québec, INSPQ, Sainte-Anne-de-Bellevue, QC; ${ }^{9}$ University of British Columbia; ${ }^{10}$ British Columbia Public Health and Microbiology Reference Laboratory, Vancouver, BC; ${ }^{11}$ Maple Leaf Foods, Mississauga, ON BACKGROUND: Listeria monocytogenes $(\mathrm{Lm})$ is a major foodborne pathogen of concern for human health and food safety in Canada. There have been several different outbreaks in Canada in the past years involving a variety of foods. Current Lm detection methods are costly and time-consuming, therefore highlighting the need to improve detection strategies.

OBJECTIVE: This study aimed to develop and validate new molecular assays for rapid identification of $\mathrm{Lm}$ based on the presence or absence of specific biomarkers.

METHODS: Whole genome sequencing was performed on $207 \mathrm{Lm}$ isolates and data was analysed using a newly developed NML-bioinformatics pipeline to identify new and unique biomarkers for incorporation into rapid real-time detection assays. Three additional biomarkers were identified from pre-existing $\mathrm{Lm}$ genome sequences. Loop-mediated isothermal amplification (LAMP) and probe based real-time PCR (qPCR) for each target were developed and tested for specificity and analytical sensitivity. The qPCR assays were also tested using different real-time platforms. Validation was performed on two types of surface areas that were artificially inoculated to determine the ability of the assays to detect Lm on surfaces as compared to the standard culture method.

RESULTS: LAMP and qPCR assays targeting three Lm-specific biomarkers, inlJ, lmo0733 and inlA and a single Listeria specific biomarker, prs were developed. The assays were capable of detecting Lm with highspecificity and sensitivity from clinical, environmental and food isolates. Non-listeriae strains were not detected based on exclusivity testing. The results on all utilized real-time platforms (ABI 7500, BioRad CFX384, Qiagen RotorGene and Roche Lightcycler 96) demonstrated equivalent detection sensitivity. Both assays were able to detect $\mathrm{Lm}$ on artificially contaminated stainless steel and plastic surfaces with $100 \%$ concordance with culture results.

CONCLUSIONS: The newly-developed LAMP and qPCR assays accurately detect $\mathrm{Lm}$ in a rapid and cost effective manner. Incorporation into routine testing will improve detection strategies for $\mathrm{Lm}$, while decreasing the result turnaround time to hours from days using the current standard Lm detection methods currently applied within food processing facilities.

\section{1:25-12:25 Oral Presentations: Session J Room: Johnson, Delta Prince Edward}

\section{J01}

IMMUNIZE ALBERTA: A MARKET-RESEARCH BASED, TARGETED, PUBLIC HEALTH IMMUNIZATION INITIATIVE TO COMBAT VACCINE HESITANCY

C Constantinescu $^{1,2}$, V Saini ${ }^{1}$, J MacDonald ${ }^{3,4}$, S Kuhn ${ }^{1,2}$, D Strong ${ }^{3,4}$, T Jadavji, ${ }^{1,2}$

${ }^{1}$ University of Calgary; ${ }^{2}$ Alberta Children's Hospital; ${ }^{3}$ Population and Public Health; ${ }^{4}$ Alberta Health Services, Calgary, AB

BACKGROUND: The control of vaccine-preventable diseases continues to be a public health challenge ${ }^{1}$. Growing vaccine-hesitancy has threatened immunization coverage and led to vaccine-preventable disease outbreaks ${ }^{2}$.

The internet provides a powerful public health tool that is currently underexploited. Messaging tailored to specific audiences is heralded as a better approach to combating vaccine- hesitancy ${ }^{3}$. We undertook to develop a targeted and tailored immunization web platform for Albertan parents using a bottom-up social marketing approach. Through market research, we identified the specific knowledge, motivation, and influencer barriers to immunization in our population. Based on this specific information, we developed a public health education tool addressing the knowledge deficiencies in our target population. It is designed to provide optimal communication with our particular population by providing the most appropriate web-based venues containing the tailored educational material.

METHODS AND RESULTS: We identified our target audience as Albertan parents with children younger than 16 years of age. A panel sample of 526 parents were interviewed and later classified into a vaccine-hesitant or vaccine-accepting group (VHG or VAG). Focusing on differences between VAG and VHG parents, we analysed data for statistically significant differences $(P<0.05)$ between groups for four domains: knowledge of immunization; public perception of immunization; motivators and barriers; immunization information sources and influencers for immunization decisions. Based on these results, two alternative approaches to web-based engagement and interaction were developed and tested in 2 focus groups interviews. Content was developed for the concept chosen, and we launched our social marketing driven immunization education initiative, ImmunizeAlberta.ca, in September 2014. The evaluation of ImmunizeAlberta as an approach for immunization education is ongoing with anticipated results available after March 2015.

CONCLUSION: ImmunizeAlberta.ca is a tailored and market-researched health-education initiative for immunization education. Our approach to its development highlights a new way to develop immunization education platforms, by harnessing appropriate web-based tools and exploiting social marketing practices to construct a targeted public health intervention

\section{J02}

\section{THE SPECIAL IMMUNIZATION CLINIC NETWORK TO INVESTIGATE PATIENTS WITH ADVERSE EVENTS FOLLOWING IMMUNIZATION AND POTENTIAL CONTRAINDICATIONS TO VACCINATION}

KA Top $^{1,2}$, G De Serres ${ }^{3}$, MC Gariepy ${ }^{3}$, SA Halperin ${ }^{1,2}$, FD Boucher ${ }^{4}$, S Dobson ${ }^{5}$, JM Pernica ${ }^{6}$, A Pham-Huy ${ }^{7}$, C Quach $^{8}$, D Tran ${ }^{9}$, W Vaudry $^{10}$, S McNeil ${ }^{1,2}$

${ }^{1}$ Canadian Center for Vaccinology, IWK Health Centre; ${ }^{2}$ Dalhousie University, Halifax, NS; ${ }^{3}$ Institut nationale du santé publique du Québec; ${ }^{4}$ Centre hospitalier universitaire de Québec, Québec, QC; ${ }^{5}$ Vaccine Evaluation Centre, BC Children's Hospital, Vancouver, BC; ${ }^{6}$ McMaster Children's Hospital, Hamilton; ${ }^{7}$ Children's Hospital of Eastern Ontario, Ottawa, ON; ${ }^{8}$ Montreal Children's Hospital, Montreal, QC; ${ }^{9}$ Hospital for Sick Children, Toronto, ON; ${ }^{10}$ Stollery Children's Hospital, Edmonton, $\mathrm{AB}$

BACKGROUND: Vaccination is a safe and highly effective public health intervention. However, for patients who experienced significant adverse events following immunization (AEFI) and for those with specific underlying medical conditions, the evidence as to how best to approach vaccination or revaccination is lacking. The Special Immunization Clinic (SIC) network was established to standardize patient management, assess outcomes after vaccination or revaccination and develop a research platform. We aim to describe the first 18 months of the network's implementation.

METHODS: SIC clinics have been established in 13 hospitals across Canada staffed by infectious diseases specialists and allergists. Inclusion criteria were: local reaction $>10 \mathrm{~cm}$, allergic symptoms $<24 \mathrm{~h}$ postimmunization, neurological symptoms, and other AEFI or medical conditions of concern. Eligible patients undergo a standardized evaluation, including causality assessment of previous AEFI, immunizations are recommended according to best practice, and AEFI are captured by telephone follow-up. Following individual consent, data are transferred to a central database for analysis.

RESULTS: As of January 2015, 122 of 142 (86\%) patients referred met the inclusion criteria. Eighty-nine patients were enrolled ( $88 \%$ of those assessed), of which $81(91 \%)$ were $<18$ years of age. Most were referred for prior AEFI (78/89, 88\%): 25 (28\%) for large local reactions, 21 (24\%) for 
allergic-like reactions, 8 (9\%) for prior neurological symptoms and 19 (21\%) for other AEFI. Eleven patients (12\%) were seen for underlying conditions that complicated vaccination. To date, $44 / 58$ (76\%) patients offered revaccination have been immunized and followed up. Nine patients $(20 \%)$ experienced adverse events; none were serious (resulted in hospitalization $>24 \mathrm{~h}$, permanent disability or death).

CONCLUSIONS: The most frequent reason for referral to a SIC are large local reactions and allergic-like events after immunization. Revaccination was safe in most patients. Through comprehensive expert assessment of patients with AEFI and contraindications to vaccination, the SIC network is helping to strengthen Canada's immunization programs.

\section{J03}

\section{USE OF WIRELESS HAND RUB DISPENSER MONITORING (EMONITORING) TO IMPROVE HAND HYGIENE ADHERENCE: A PILOT STUDY}

D MacFadden, C Moore, J So, A Cohen, L Wayment, A Yirenkyi, L Zhang, W Lee, D Willcocks, L Maze Dit Mieusement, E Thomas, J Wong, A O'Shaughnessy, L McCreight, AJ McGeer Mount Sinai Hospital, Toronto, ON

BACKGROUND: Appropriate hand hygiene among healthcare workers is necessary for reducing healthcare associated infection. Systems providing electronic monitoring (eMonitoring) and feedback of hand hygiene adherence have the potential to support improvements in practice.

METHODS: We performed a time-series study of the introduction of eMonitoring (DebMed®) with near real-time feedback on three medical/ surgical wards at an academic hospital. Rates of hand hygiene adherence were monitored electronically and by observational audit during the preintervention period (December 12013 to February 14 2014), intervention period (February 15 to May 31 2014), early post-intervention (June 1 to August 31 2014), and late post-intervention (September 1 to November 30 2014). During the intervention, units selected the method of feedback from the eMonitoring system. Hand hygiene adherence was measured with eMonitoring as a hand hygiene compliance index (HHCI), representing the number of dispenses of handrub divided by the number of expected hygiene opportunities per patient hour (percentage).

RESULTS: Observational audit adherence and eMonitoring HHCI across the three units for Pre/During/Early Post/Late Post-Intervention periods were: Unit 1 ( $85 \% / 88 \% / 89 \% / 88 \%$ by audit, $24 \% / 30 \% / 33 \% / 28 \%$ by eMonitor), Unit 2 (94\%/89\%/86\%/95\% by audit, 24\%/36\%/31\%/33\% by eMonitor), and Unit 3 (92\%/86\%/92\%/94\% by audit, 39\%/48\%/44\%/44\% by eMonitor). The overall eMonitoring $\mathrm{HHCI}$ increased from pre to during intervention periods ( $29 \%$ to $38 \%, \mathrm{P}<0.001$ ), decreased from during to early post-intervention periods ( $38 \%$ to $36 \%, \mathrm{P}<0.001$ ), and decreased from early to late post-intervention periods ( $36 \%$ to $35 \%, \mathrm{P}<0.001)$. Observed audit adherence rates did not appear to significantly change from pre to during intervention periods ( $90 \%$ to $87 \%, \mathrm{P}=0.21$ ), from during to early post-intervention periods ( $87 \%$ to $89 \%, \mathrm{P}=0.12$ ), or from early to late post-intervention periods ( $89 \%$ to $92 \%, \mathrm{P}=0.24$ ).

CONCLUSIONS: eMonitoring of hand hygiene with feedback improved hand hygiene adherence and may be less susceptible to biases inherent in observational audits. Continued feedback may be necessary to sustain performance.

\section{J04}

\section{\#INSTAGRAM+ID+ME: INCREASING STUDENT ENGAGEMENT WITH AN INFECTIOUS DISEASES LEARNING TOOL ON PHOTOGRAPHY BASED SOCIAL MEDIA}

\section{Wood, E Forgie, $\underline{S \text { Forgie }}$}

University of Alberta, Edmonton, AB

OBJECTIVE: We created a virtual photo album of infectious diseases (ID) memory hangers (visual mnemonics with short explanations) on Instagram (C), an online, photography based social media platform, to accompany an ID course in 2013. Students who used it found the album was useful for review and the flexible format was helpful. However, student engagement was limited to less than $1 / 3$ of the class. This year we recreated the album and tried to promote student engagement by also using relevant news stories, asking questions, and by challenging students to create their own memory hangers.

METHODS: Ethics approval was obtained and a private Instagram@ account was created. Each memory hanger was uploaded synchronously after the corresponding face-to-face sessions. Questions and links to current news stories about the memory hangers were also uploaded over time. Instagram $(\bigodot$ allowed students to access the album from their mobile phones, receive push notifications about new postings, add comments, 'like' them or link out to their accounts and display their own memory hangers. The tool was then evaluated with usage analysis.

RESULTS: Seventy-six ID memory hangers were uploaded during the course (vs. 35 in 2013) and 8 more about ID issues in the news were uploaded after the course. 170 students (vs. 58 in 2013) followed the account. There were 157 likes on 68 different memory hangers. Based on the number of likes, "Things I am paranoid about after the course", H. pylori, Harry Houdini and the Enteric Jazz Band and the Penicillin Pyramid were the most popular. Nine postings had multiple student comments related to questions (vs. 2 in 2013), 17 student groups created memory hangers (vs. 0 in 2013), and 2 memory hangers were created by students after the course (C. difficile and a rap about Hip Hop Haemophilus and Rhymnovirus). There were 81 likes of the 8 memory hangers posted after the course and 2 had $>1$ comment.

CONCLUSIONS: Student engagement with the Instagram ID memory hangers album increased this year with our interventions. Many students have remained engaged after the completion of the course through creation of their own memory hangers or through commenting on items we have posted related to current ID events in the news.

\section{J05}

\section{COMMUNITY-BASED INFECTIOUS DISEASE CLINICS: A TOOL OF ENGAGEMENT FOR VULNERABLE POPULATIONS}

B Conway, S Hakobyan, G Kerkerian, S Sharma, S Tahmasebi,

\section{H Tossonian}

Vancouver Infectious Diseases Centre, Vancouver, BC

BACKGROUND: As we seek to implement more effective treatment modalities for HIV and HCV infection in all target populations, novel approaches to engagement in care must be considered to ensure to maximize individual success and impact the spread of disease.

METHODS: We have designed and evaluated the Community Pop-Up Clinic (CPC) model on the Downtown East Side of Vancouver. The clinic is held at a community-based site on a weekly basis and accepts up to 30 patients/event. It is staffed by 6 individuals, including an infectious disease specialist. Point-of-care testing for HIV and HCV is offered, as well as review of existing infection status, and design of a plan of engagement in care. Follow-up is offered at the established clinic, as a walk-in or by appointment. Patients are also invited to fill out a questionnaire since January 2014 to record demographic information and knowledge of disease state.

RESULTS: Since 2013, 1025 patients have been tested during CPCs, 305 HCV positive (29.8\%), 240 Male (78.7\%), 22 co-infected with HIV (2.1\%), $6 \mathrm{HIV}$ positive $(0.6 \%)$. Out of 311,80 people showed up at least once, $7 \mathrm{HIV}$ positive patients are now on antiretroviral therapy at VIDC, with $57 \%$ having an undetectable viral load. We have treated 14 patients for HCV infection, 7 with all-oral regimens. The SVR rate is $86 \%$, with 3 more patients awaiting the initiation of HCV therapy in the coming 3 months.

CONCLUSIONS: The CPC model has proven highly effective for the identification and engagement in care of vulnerable individuals infected with HIV and HCV, many of whom were not engaged despite multiple previous attempts. CPC will be a powerful tool for addressing the dual HIV and HCV epidemics in Canada, with particular impact on treating those who are most likely to be core transmitters of HCV and HIV. 
11:25-12:25 Oral Presentations: Session K Room: Brown, Delta Prince Edward

\section{K01}

PCR DETECTION OF CARBAPENEMASE-PRODUCING
ORGANISMS (CPO) USING CEPHEID'S XPERT CARBA-R
(XCR) VERSION 1 AND 2 ASSAYS
BM Willey ${ }^{1}$, DA Boyd ${ }^{2}$, G Ricci ${ }^{3}$, X Trimi ${ }^{4}$, P Rahman ${ }^{1}$, DN Grohn ${ }^{5}$, O Leung ${ }^{6}$, S Dunna ${ }^{4}$, P Lo ${ }^{1}$, MR Mulvey ${ }^{2}$, T Mazzulli ${ }^{1,4}$, SM Poutanen ${ }^{1,4}$

${ }^{1}$ Mount Sinai Hospital/University Health Network, Toronto, ON; ${ }^{2}$ National Microbiology Laboratory, Winnipeg, MB; ${ }^{3}$ William Osler Health Sciences Centre, Brampton; ${ }^{4}$ University of Toronto; ${ }^{5}$ Michener Institute, Toronto; ${ }^{6}$ University of Western Ontario, London, ON

OBJECTIVES: Timely detection and confirmation of CPO is integral to effective infection prevention and dissemination control critical to patient care. Most laboratories are not able to perform conventional PCR on suspected CPO. Using a comprehensive collection of retrospective clinical isolates, this study verified version 1 ( $v 1$ ) of the low-complexity XCR assay which claims to detect IMP1, KPC, NDM, OXA48-like and VIM genes. Additional tests were performed on a subset of $\mathrm{CPO}$ and non-CPO following the initial study using XCR version 2 (v2) after modifications were made to improve OXA48-like gene detection.

METHODS: 228 test Gram-negative bacilli (mostly Enterobacteriaceae) included $194 \mathrm{CPO}$ and 34 non-CPO isolates. Only $187 \mathrm{CPO}$ were expected to be detected by XCR (100 KPC, 53 NDM, 13 OXA48, 6 VIM, 6 OXA181, 3 OXA232, 3 OXA181, 2 OXA232, 1 OXA244) as genes in 7 CPO (3 SME, 2 NMC, 1 IMP7, 1 OXA24) were not claimed, and the reduced-susceptibility to carbapenems in 32 non-CPO was due to other mechanisms [ESBL, ampC-hyperproduction, porin mutations]. It was unclear how XCR would perform for a Shewanella (chromosomal OXA252) and an NDM-revertant. $\mathrm{XCR}$ was run as per Cepheid except that buffers were inoculated from lightly touched isolates and not specimens.

RESULTS: XCR v1 detected 100\% of KPC (95\% CI: 96-100), NDM (95\% CI: 93-100), VIM (95\% CI: 56-100), OXA48-specific (95\% CI: 73-100), OXA244 (95\% CI;22-100) CPO and the OXA252 progenitor gene. 0/14 OXA48-like genes were detected (0/9 OXA181 and 0/5 OXA232). XCR was negative for SME, NMC, OXA24 and all non$\mathrm{CPO}$ and the NDM-revertant. Overall sensitivity $(\mathrm{Sn}) /$ specificity $(\mathrm{Sp})$ for claimed genes was $92 \%$ (95\% CI: 88-96)/98\% (95\% CI: 86->99.9). Testing on XCR v2 detected all OXA48 and OXA48-like genotypes, resulting in an overall Sn of 100\% (95\% CI: 98-100) for claimed CPO genes.

CONCLUSIONS: The XCR v1 was simple to perform, providing highly accurate $(100 \%) \mathrm{CPO}$ results in $1 \mathrm{~h}$ for claimed KPC, NDM, VIM and OXA48-specific genes. Modifications made in v2 enabled XCR to detect the increasingly more common OXA48-like genotypes (OXA181 and OXA232) that vary from OXA48 by only minor sequence mutations. The XCR v2, currently a research-use-only assay, has been submitted to FDA for release as an IVD assay to enable use from clinical specimens for detecting the listed CPO genotypes.

\section{K02}

COMPARISON OF THE ABACUS DIAGNOSTICA GENOM ERA CDX ${ }^{\mathrm{TM}}$ (CDX) PCR AND THE FOCUS DIAGNOSTIC SIMPLEXA ${ }^{\text {TM }}$ UNIVERSAL DIRECT C. DIFFICILE (FCD) PCR FOR THE DIAGNOSIS OF C. DIFFICILE INFECTION (CDI)

I Gorn², E Adam²,3, M Desjardins ${ }^{1,2}$

${ }^{1}$ Eastern Ontario Regional Laboratory; ${ }^{2}$ Ottawa Research Institute, ${ }^{3}$ University of Ottawa, Ottawa, ON

OBJECTIVE: The CDX direct PCR for the diagnosis of CDI is a rapid and new amplification system that detects the C. difficile (CD) tcdB gene. The purpose of this study was to evaluate the performance of the CDX assay compared to the Simplexa FCD assay for the direct detection of C. difficile in stool samples.
METHOD: Glutamate Dehydrogenase (GDH) positive and negative samples were tested by PCR using the CDX and FCM assays according to manufacturer's instructions. All samples were also cultured using the CHROMagar ${ }^{\mathrm{TM}} \mathrm{CD}$ fluorogenic chromogenic agar (CHROM). Colonies suspicious for CD were identified using MALDI-TOF (Bruker). Cultures positive for $\mathrm{CD}$ with corresponding negative PCRs were confirmed to be toxigenic by testing isolates for the tcdB gene using the FCD PCR. A CD positive result was defined as a sample positive by toxigenic culture or positive by GDH and both the FCD and CDX PCRs.

RESULTS: Of the 148 samples tested, 63 and 85 met the definition of a $\mathrm{CD}$ positive and $\mathrm{CD}$ negative sample respectively. Of the $63 \mathrm{CD}$ positive samples, 43 (68\%) were positive by culture and both the CDX and FDC assays. There were 101 samples negative by culture, 16 (16\%) of these were positive by GDH, FDC and CDX PCR. We tested 34 GDH negative samples and all were negative by culture and both PCRs. The sensitivity, specificity, negative and positive predictive values of the CDX and FDC assays were $94 \%, 96 \%, 95 \%, 95 \%$ and $95 \%, 98 \%, 97 \%, 96 \%$, respectively.

CONCLUSION: The performance of both amplification methods was very similar. Toxigenic cultures using the fluorogenic CHROM agar failed to identify CD in $16 \%$ of samples positive by both PCRs and many fluorogenic colonies did not confirm as CD. Although this selective media can be used for epidemiological purposes, the high rate of false negatives and low specificity may not be suitable for diagnostic purposes. Overall the Abacus CDX assay provides an accurate, rapid and simple to use alternative amplification method for the diagnosis of CDI.

\section{K03}

DETECTION OF GROUP B STREPTOCOCCI (GBS)

DIRECTLY FROM E-SWABS BY ISOTHERMAL

AMPLIFICATION: AN AFFORDABLE, AND RAPID POINTOF-CARE ALTERNATIVE TO EXPENSIVE COMMERCIAL MOLECULAR DIAGNOSTIC TESTS

\section{P Jayaratne $\mathrm{e}^{1,2,3}$, F Al Mutawa ${ }^{2}$}

${ }^{1}$ St Joseph's Healthcare; ${ }^{2}$ McMaster University; ${ }^{3}$ Hamilton Health Sciences, Hamilton, ON

OBJECTIVES: GBS is a leading cause of neonatal morbidity and mortality. It is the most common life threatening sepsis and meningitis in neonates especially the first week of life. It can be prevented if the mother diagnosed during antenatal period and treated at labor. Current guidelines recommend that all pregnant women be screened for vaginal/rectal colonization at 35-37 weeks of gestation. Rapid commercial molecular tests are too expensive to be used in GBS screening. The objective of this study was to develop an accurate method that is comparable in performance to culture but faster and cheaper. We evaluated the performance of LAMP assay to detect GBS colonization in the anogenital tract of pregnant women using E-swab specimens and compared to culture.

METHOD: Randomly selected E-swab specimens from 275 women from the period January 2014 to September 2014 sent for routine screening at Hamilton Health science was used. For LAMP assay E-swab fluid was concentrated and added to a lysis solution prior to boiling for $15 \mathrm{~min}$ to obtain a crude extract of nucleic acids. For LAMP 210bp fragment of $c f b$ gene of GBS was amplified using primers designed by Primer Explorer V4 software (Eiken Chemical Co). LAMP was carried out at $65^{\circ} \mathrm{C}$ for $30 \mathrm{~min}$ using a standard reaction mixture containing Bst 2.0 WS DNA polymerase (New England BioLabs). LAMP amplification was detected using Genie ${ }^{\circledR}$ II (OptiGene, UK). For culture $30 \mu \mathrm{L}$ of E-swab fluid was inoculated into LIM broth and incubated overnight at $35^{\circ} \mathrm{C}$ followed by plating on Chromoagar for 24 hour before examining the plate.

RESULTS: Sixty four specimens were GBS positive by both culture and LAMP. There were 4 discordant results of which 2 were culture positive and LAMP negative and two were LAMP positive and culture negative. The performance characteristics of LAMP as compared to culture were as follows: 97\% sensitivity, 99\% specificity, 97\% PPV and 99\% NPV. The turn-around-time for LAMP was $60 \mathrm{~min}$ as compared to $\geq 48$ hours for culture. The cost for LAMP is about Cdn $\$ 3.00$ per test.

CONCLUSION: Detection of GBS by LAMP using the amplification of $c f b$ gene is rapid and cost-effective than culture with comparable performance to commercial molecular tests. This assay provides promising results for rapid detection of GBS during screening. 


\section{K04}

DETECTION OF GROUP B STREPTOCOCCUS (GBS) FROM VAGINAL-RECTAL SCREENING SPECIMENS BY LOOP MEDIATED AMPLIFICATION (ILLUMIGENE® GBS) ASSAY FROM TWO BROTHS

P Lagacé-Wiens $^{1}{ }^{1,2}$, B Hosseini ${ }^{2}$, G Poliquin², S Edagiz ${ }^{2}$, JA Karlowsky ${ }^{1,2}$ ${ }^{1}$ Diagnostic Services Manitoba; ${ }^{2}$ University of Manitoba, Winnipeg, MB

BACKGROUND: GBS is a common cause of early-onset neonatal sepsis. Widespread use of GBS screening and antibiotic prophylaxis has reduced the incidence of disease significantly. Technical improvements in screening may result in further reductions in disease prevalence. One method of reducing turn-around time for detection of GBS without compromising sensitivity is direct detection of GBS from enrichments broths using convenient, affordable and random-access molecular platforms. One such platform is the illumigene ${ }^{\circledR}$ GBS assay, which uses loop-mediated amplification technology to detect GBS in selective broths after overnight broth enrichment. The purpose of this study was to assess the sensitivity and specificity of GBS detection from two types of enrichment broths after overnight incubation of pre-natal vaginal-rectal swabs.

METHODS: Vaginal-rectal swabs were collected in duplicate from women attending routine pre-natal appointments between 34 and 36 weeks gestation. Swabs were placed in either Todd-Hewitt (TH) broth $\left(\right.$ Oxoid $^{\mathrm{TM}}$ ) or RAMBAQuick (RQ) broth (CHROMagar ${ }^{\mathrm{TM}}$ ), which is designed to reduce overgrowth of enterococci isolated in vaginal-rectal samples. Broths were incubated overnight at $35^{\circ} \mathrm{C}$. GBS was detected using the illumigene ${ }^{\circledR}$ GBS assay following manufacturer's instructions (off-label use for RQ broth). was Broths were sub-cultured to sheep blood agar (SBA) and Colorex ${ }^{\mathrm{TM}}$ StrepB (CSB, CHROMagar ${ }^{\mathrm{TM}}$ ). GBS were identified using colony morphology and Lancefield typing from SBA and by chromogenic reaction and Lancefield typing from CSB. GBS growth from the broth on either SBA or CSB was considered the gold standard. RESULTS: 45 of 204 samples were positive for GBS in TH broth by culture and 44 were positive in RQ broth by culture. Sensitivity of illumigene ${ }^{\circledR}$ was 97.7\% (43/44) from the RQ broth and 100\% (45/45) from the TH broth. Specificity of the iilumigene ${ }^{\circledR}$ assay was 100\% for both broths and an invalid illumigene ${ }^{\circledR}$ result occurred in $1(0.2 \%)$ test.

CONCLUSION: illumigene ${ }^{\circledR}$ performed equally well from both broths and had very high sensitivity and specificity. The illumigene ${ }^{\circledR}$ assay was easy to perform with minimal training or expertise and provided rapid results without the need for colony picking or additional testing (e.g. Lancefield typing). Turnaround time was reduced by one day compared to conventional broth-enrichment culture.

\section{K05}

COMPARATIVE EVALUATIONS OF COMMERCIALLY AVAILABLE RAPID PHENOTYPIC TESTS FOR DETECTING CARBAPENEMASE-PRODUCING ORGANISMS (CPO)

BM Willey ${ }^{1}$, X Trimi ${ }^{2}$, P Rahman ${ }^{1}$, DN Grohn ${ }^{3}$, DA Boyd ${ }^{4}$, G Ricci ${ }^{5}$, O Leung $^{6}$, S Dunna ${ }^{2}$, P Lo $^{1}$, T Mazzulli $^{1,2}$, SM Poutanen ${ }^{1,2}$

${ }^{1}$ Mount Sinai Hospital/University Health Network; ${ }^{2}$ University of Toronto; ${ }^{3}$ Michener Institute, Toronto, $\mathrm{ON}$; ${ }^{4} \mathrm{National}$ Microbiology Laboratory, Winnipeg, MB; ${ }^{5}$ William Osler Health Sciences Centre, Brampton; ${ }^{6}$ University of Western Ontario, London, $\mathrm{ON}$

OBJECTIVES: While molecular tests cannot target every CPO, the phenotypic Carba-NP (CNP) claims to rapidly distinguish all CPO from nonCPO. But many studies have found the CNP insensitive for OXA48-like CPO. This study compared capabilities of similar $\mathrm{CPO}$ detection tests from Rosco [Carba Blue (CBL); Rapid Carba Screen (RCS); modified RCS (mRCS)] and bioMérieux [Rapidec Carba-NP (RCN)] using characterized clinical strains.

METHODS: $206 \mathrm{Gram}$-negative bacilli (mostly enterobacteria) were randomized for testing. These included 175 CPO [91 KPC, 49 NDM, 10 OXA48, 6 OXA181, 6 VIM, 3 OXA232, 2 NDM+OXA181, 2 NMC, 2 SME, 1 IMP7, 1 NDM+OXA232, 1 OXA24, 1 OXA244] and 31 non$\mathrm{CPO}$ (variously carbapenem-insusceptible ESBL, ampC or OMP-mutants). $\mathrm{RCN}, \mathrm{CBL}$ and RSC were tested and interpreted as per manufacturer while
mRCS used $0.8 \%$ saline $(\mathrm{pH} 8.0)$ in lieu of extraction buffer. Compared to controls, positive endpoints at 15, 30,60 and 120 min of yellow or orange $(\mathrm{Y} / \mathrm{O})$, or $\mathrm{Y} / \mathrm{O} /$ red-orange $(\mathrm{Y} / \mathrm{O} / \mathrm{RO})$ for $\mathrm{RCN}, \mathrm{RCS}$, and $\mathrm{mRCS}$ and $\mathrm{Y} / \mathrm{green}$ $(\mathrm{Y} / \mathrm{G})$ or $\mathrm{Y} / \mathrm{G} / \mathrm{blue}$-green $(\mathrm{Y} / \mathrm{G} / \mathrm{BG})$ for $\mathrm{CBL}$, respectively, were noted.

RESULTS: The RCN detected $100 \%$ non-OXA CPO but only with criteria of $\mathrm{Y} / \mathrm{O} / \mathrm{RO}$ at $120 \mathrm{~min}$. Using these criteria, 77\% OXA CPO went undetected and $12.9 \%$ of non-CPO were erroneously pos. If an RCN endpoint of $\mathrm{Y} / \mathrm{O}$ at $120 \mathrm{~min}$ was applied, detection of non-OXA CPO was reduced to $97.4 \%$ and OXA CPO to $0 \%$ but $0 \%$ false-pos were incurred. Using an endpoint of $\mathrm{Y} / \mathrm{O}$ at $120 \mathrm{~min}$, the mRCS outperformed RCS, detecting $99.4 \%$ vs $79.9 \%$ non-OXA CPO and $42.9 \%$ vs $10.5 \%$ OXA, respectively, with no false-pos calls for non-CPO strains. For CBL, if an endpoint of $\mathrm{Y} / \mathrm{G}$ at $120 \mathrm{~min}$ was used, non-OXA CPO detection was $98.7 \%$ and OXA-CPO detection was $42.9 \%$, but $9.7 \%$ non-CPO were also incorrectly called pos. Including $\mathrm{Y} / \mathrm{G} / \mathrm{BG}$ endpoints at 30,60 or $120 \mathrm{~min}$ increased OXA-CPO detection to $71.4 \%, 66.6 \%$ and $66.6 \%$, respectively, but also substantially increased the false-pos rates to $51.6 \%$, $48.4 \%$ and $35.5 \%$, respectively.

CONCLUSIONS: No assay detected all CPO despite modifications or consideration of various pos colour endpoints. While these assays are valuable when definite colour changes are obtained, laboratories must be aware that none work alone to detect all $\mathrm{CPO}$ and most have associated false-pos, especially when weaker colour-changes are considered pos.

\section{1:25-12:25 Oral Presentations: Session L Room: Chandler, Delta Prince Edward}

\section{L01}

\section{ANTIBIOTIC RESISTANCE SURVEILLANCE FOR THE COMMUNITY: CLOSING THE GAP THROUGH PUBLIC- PRIVATE COLLABORATION IN BC}

D Patrick ${ }^{1,2}$, D George ${ }^{2}$, R Reyes ${ }^{1,3}$, D Purych ${ }^{1,3}$, MR Mulvey $^{4}$, R Finley ${ }^{5}$, M Kelly ${ }^{1,3}$

${ }^{1}$ University of British Columbia; ${ }^{2}$ British Columbia Centre for Disease Control, Vancouver; ${ }^{3}$ LifeLabs Medical Laboratory Services, Burnaby, BC; ${ }^{4}$ National Microbiology Laboratory, Winnipeg, MB; ${ }^{5}$ Public Health Agency of Canada, Guelph, ON

OBJECTIVES: A review of antibiotic resistant organisms (ARO) surveillance in Canada revealed a gap outside of the hospital setting. We hypothesized that, where a large proportion of susceptibility testing is concentrated in a single laboratory network, collaboration between the providing laboratory and surveillance units could fill the void.

METHODS: LifeLabs collects specimens from 80 community-based patient service centres located throughout British Columbia (BC) representing approximately $80 \%$ of community-based testing and is also well represented in other provinces. Steps in collaboration with the BC Do Bugs Need Drugs program at BCCDC included proposal review, datasharing agreement, extension of a three-party non-disclosure agreement to include work with the Public Health Agency of Canada (PHAC), data transfer to a secure server, analysis and collaborative report production involving surveillance staff and LifeLabs microbiologists.

RESULTS: The entire process took four months, with about $80 \%$ of the time being required to finalize agreements. Data from 2007-2013 were provided and included 744,155 records from two information systems, 121,732 records for 2013 alone. Data structure included age-group, sex, local health area, date of collection, anatomical site, organism and variables for each antibiotic of interest. Extraction from a reporting database meant some limitation in organism-drug combinations and a need to assure denominators were accurately accrued. The process allowed reporting for 14 organisms of interest, detailed analysis of trends by age-group, gender and region within the province and provided very large sample sizes for robust confidence intervals. The process appears sustainable through twice-yearly data extraction.

CONCLUSIONS: The public private collaboration model makes use of existing data, avoids the costs of de novo surveillance and is easily achieved when supported by laboratory leadership. Its application to other regions 
will be explored with PHAC. In-depth analysis (e.g by age, region and over time) can provide professionals with new tools to combat AROs.

\section{L02}

ANTIFUNGAL SUSCEPTIBILITY OF RESPIRATORY ASPERGILLUS ISOLATES FROM CANADIAN HOSPITALS: RESULTS OF THE CANWARD 2014 STUDY

Luller $^{1}$, S Shokoples ${ }^{1}$, L Turnbull ${ }^{1}$, RP Rennie ${ }^{1}$, HJ Adam² ${ }^{2}$, M Baxter $^{2}$, DJ Hoban², GG Zhanel ${ }^{2}$

${ }^{1}$ Provincial Laboratory, Alberta Health Services, University of Alberta, Edmonton, AB; ${ }^{2}$ Medical Microbiology and Infectious Diseases, University of Manitoba, Winnipeg, MB

OBJECTIVES: CANWARD is an ongoing national surveillance study that assesses pathogens causing infections in patients attending Canadian hospitals, as well as determines the prevalence of antimicrobial resistance in these isolates. Here we present the distribution of species and in vitro antifungal susceptibility of Aspergillus isolates collected in 2014 from patients visiting Canadian hospitals.

METHODS: Clinical Aspergillus isolates recovered from respiratory specimens at 13 participating medical centres during the 2014 study period were tested against amphotericin B (AMB), itraconazole (ITRA), posaconazole (POSA), voriconazole (VORI) and caspofungin (CASP) by broth microdilution using the CLSI M38-A2 method. Growth endpoints were measured as per CLSI M38-A2 and values above the published epidemiological cutoff values $(\mathrm{mg} / \mathrm{L})$ were scored as non-wildtype (non-WT). Clinical breakpoints are not available for Aspergillus susceptibility interpretation.

RESULTS: Of the 713 Aspergillus isolates recovered, A. fumigatus, A. niger, A. flavus, and A.terreus represented $71.9 \%, 11.2 \%, 6.5 \%$, and $3.9 \%$ of the population, respectively. A. fumigatus isolates were recovered primarily from Clinic outpatients (68\%) and Medicine inpatients (19\%) from sputa (62\%) and bronchoscopy (30\%) specimens. Very few Aspergillus isolates exhibited non-WT MIC and MEC values. Twenty isolates (4\%) of A. fumigatus had non-WT VORI MICs (2 to $4 \mathrm{mg} / \mathrm{L}$ ) and 5\% of A. niger had non-WT MICs to VORI, ITRA, or POSA. There was very little evidence of microbiological resistance to echinocandins across the species. Six A. calidoustus isolates from six centres were recovered, all exhibiting non-WT MICs to the azoles and variably high MECs to caspofungin.

CONCLUSION: Epidemiological cutoff values have been published to aid the detection of acquired microbiological resistance in Aspergillus. In this study, WT isolates of A. fumigatus were most prevalent and the rate of non-WT isolates was very low and comparable to rates from the previous two years of surveillance. Intrinsically resistant species like A. calidoustus remain very uncommon in respiratory cultures.

\section{L03}

\section{ANTIMICROBIAL RESISTANCE IN PATHOGENS ISOLATED} FROM CANADIAN HOSPITALS: CANWARD 2014

M Baxter $^{1}$, HJ Adam ${ }^{1,2}$, D Spreitzer ${ }^{3}$, A Denisuik ${ }^{1}$, G Golding ${ }^{1,3}$, JA Karlowsky ${ }^{1,2}$, DJ Hoban ${ }^{1,2}$, GG Zhanel ${ }^{1}$

${ }^{1}$ University of Manitoba; ${ }^{2}$ Diagnostic Services Manitoba;

${ }^{3}$ National Microbiology Laboratory, Winnipeg, MB

OBJECTIVES: The CANWARD study assesses the pathogens causing infections in patients affiliated with Canadian hospitals, and evaluates the prevalence of antimicrobial resistance in these isolates.

METHODS: Thirteen tertiary-care centres across Canada submitted pathogens causing infections from patients attending clinics $(\mathrm{C})$, emergency rooms (ER), medical and surgical wards (W) and intensive care units (ICU) in 2014. Susceptibility testing was performed by CLSI broth microdilution methods.

RESULTS: A total of 3,196 isolates were collected: 40.0\%, 40.2\%, 10.1\%, and $9.7 \%$ from blood, respiratory, urine and wound/IV site specimens, respectively. Patient demographics were as follows: 56.4/43.6\% male/female, $13.8 \% \leq 17$ years, $42.3 \% 18-64$ years and $43.9 \% \geq 65$ years. Isolates were from patients on W $39.5 \%$, ER $23.3 \%$, ICU $18.8 \%$, and C $18.4 \%$. The most common pathogens were: E. coli 19.6\%, S. aureus (MSSA) 19.5\%, P. aeruginosa $11.0 \%$, K. pneumoniae $5.8 \%$, S. aureus (MRSA) $5.0 \%$, and S. pneumoniae $4.9 \%$. Resistance rates for E. coli were: $0 \%$ for meropenem and tigecycline,
$0.3 \%$ ertapenem, $1.6 \%$ piperacillin/tazobactam, $9.6 \%$ gentamicin, $12.0 \%$ ceftriaxone, $24.4 \%$ ciprofloxacin and $28.8 \%$ trimethoprim/sulfamethoxazole (SXT). For P. aeruginosa, resistance rates were $1.1 \%$ colistin, $7.1 \%$ gentamicin, $8.6 \%$ piperacillin-tazobactam, $16.6 \%$ meropenem, and $17.7 \%$ ciprofloxacin. Resistance rates for MRSA were: $0 \%$ vancomycin, daptomycin and linezolid, 5.0\% tigecycline, 5.0\% SXT, 38.7\% clindamycin, $80.0 \%$ ciprofloxacin, and $82.5 \%$ clarithromycin. Overall, the prevalence of MRSA and vancomycin-resistant enterococci (VRE) was $20.5 \%$ and $7.6 \%$, respectively. CONCLUSIONS: In Canada, resistance rates for E. coli remain lowest for meropenem, tigecycline, ertapenem and piperacillin-tazobactam, while for P. aeruginosa, rates are lowest with colistin, gentamicin, and piperacillintazobactam. No resistance was observed in MRSA with vancomycin, linezolid, or daptomycin.

\section{L04}

ANTIMICROBIAL RESISTANT ORGANISMS IN CANADA: JANUARY 1, 2009 TO DECEMBER 31, 2013

R Mitchell, L Pelude, S Leduc, K Choi, K Abdesselam, C Archibald, D Gravel, $\underline{C}$ Frenette

Public Health Agency of Canada, Ottawa, ON

BACKGROUND: Surveillance of antimicrobial resistant organisms (AROs) in hospitalized patients is vital to informing strategies to reduce the risks and impacts of antimicrobial resistance.

METHODS: The Public Health Agency of Canada collects national data on AROs in hospitalized patients through the Canadian Nosocomial Infection Surveillance Program (CNISP). As of December 2013, CNISP conducted surveillance in 59 major acute-care hospitals in Canada.

RESULTS: Canadian healthcare-associated Clostridium difficile infection (HA-CDI) rates remained stable from 2009 (5.81 per 10,000 patient days) to 2013 (5.36 per 10,000 patient-days). HA-CDI attributable mortality rates peaked in $2011(6.4 \%)$ and fell by $52 \%$ in $2013(3.1 \%)$. National methicillin-resistant Staphylococcus aureus (MRSA) infection rates have decreased since 2009, with the most dramatic reduction seen in healthcare-associated rates (2.56 to 1.77 per 10,000 patient days). Vancomycin-resistant enterococci (VRE) infection rates in Canada remain low but have been slowly increasing since 2009 ( 0.32 to 0.54 per 10,000 patient days). Regional variations exist with the highest rates in the western region ( 0.79 per 10,000 patient days) and lowest in the east (0.08 per 10,000 patient days). Rates of carbapenemase-producing organism (CPO) and carbapenem-resistant enterobacteriaceae (CRE) in CNISP participating hospitals remain low. The central region of Canada reported the highest number of $\mathrm{CRE}$ and $\mathrm{CPO}$ cases, the majority of which are likely due to an outbreak at one hospital.

CONCLUSIONS: In Canada, ARO rates among hospitalized patients have been relatively stable since 2009. Regional variations have been reported and may be partially explained by differences in the virulence of circulating strains, infection control practices, antimicrobial stewardship programs and surveillance and screening activities. These data highlight the importance of continuing to monitor trends to ensure the detection of new and re-emerging threats associated with antimicrobial resistance.

L05

ANTIFUNGAL SUSCEPTIBILITY OF INVASIVE CANDIDA ISOLATES FROM CANADIAN HOSPITALS: RESULTS OF THE CANWARD 2014 STUDY

L Fuller ${ }^{1}$, S Shokoples ${ }^{1}$, L Turnbull ${ }^{1}$, RP Rennie ${ }^{1}$, HJ Adam ${ }^{2}$, M Baxter $^{2}$, DJ Hoban ${ }^{2}$, GG Zhanel ${ }^{2}$

${ }^{1}$ Provincial Laboratory, Alberta Health Services, University of Alberta, Edmonton, AB; ${ }^{2}$ Medical Microbiology and Infectious Diseases, University of Manitoba, Winnipeg, MB

OBJECTIVE: CANWARD is an ongoing national surveillance study that assesses pathogens causing infections in patients admitted to Canadian hospitals, as well as determines the prevalence of antimicrobial resistance (R) in these isolates. Here we present the antifungal susceptibility data for candidemia isolates collected in 2014.

METHODS: Candida species isolated from bloodstream infections were collected from 12 participating medical centres during the 2014 study 
period. Antifungal susceptibility testing and interpretation was performed as per CLSI M27-S4 broth microdilution method and interpretation guidelines for fluconazole (FLUC), caspofungin (CASP), and micafungin (MICA). Epidemiological cut-off values of $\leq 2 \mathrm{mg} / \mathrm{L}$ for amphotericin B $(\mathrm{AMB})$ and $0.5 \mathrm{mg} / \mathrm{L}$ for voriconazole (VORI) against C. glabrata (CG) were used in the absence of M27 breakpoints.

RESULTS: Of 328 Candida spp. collected, C. albicans (CA) was predominant $(48.8 \%)$, followed by C. glabrata (CG, $22.0 \%)$, C. parapsilosis (CP, $9.8 \%)$, and C. tropicalis $(6.1 \%)$. The majority of cases were identified in ICU (31.4\%), medicine (36.3\%), and surgical wards (15.6\%). Susceptibility (S) values are in table below and limited acquired resistance was detected. High level azole $\mathrm{R}$ was detected in one $\mathrm{CA}$ and two CP isolates. Echinocandin R was detected in four CG isolates (5.6\%), all of which contained fks mutations.

\begin{tabular}{|l|l|llllll|}
\hline \multirow{2}{*}{} & & \multicolumn{2}{|l}{ MIC $_{90}(\% \mathrm{R})$} & & & & \\
ID & No. & AMB & ITRA & FLUC & VORI & CASP & MICA \\
\hline CA & 160 & $0.5(0)$ & 0.06 & 0.5 & 0.03 & 0.12 & $<0.007$ \\
& & & $(0.6)$ & $(0.6)$ & $(0.6)$ & $(0.6)$ & $(0.6)$ \\
CG & \multirow{2}{*}{72} & $0.5(0)$ & $0.5(-)$ & $8\left(0^{\star}\right)$ & 0.25 & not & 0.03 \\
& & & & & $(2.8)$ & tested & $(5.6)$ \\
CP & \multirow{2}{*}{32} & $1.0(0)$ & $0.12(-)$ & $2.0(0)$ & $0.06(0)$ & $1.0(0)$ & $1.0(0)$ \\
\hline
\end{tabular}

*Susceptible dose-dependent; - No clinical breakpoint; ITRA Itraconazole;

CONCLUSION: CANWARD surveillance of invasive Candida isolates since 2011 shows stable distribution of species and antifungal MICs in hospitalized patients, and limited evidence of resistance. C. glabrata is a common cause of candidemia and echinocandin resistance of $\sim 5 \%$ in Canada is associated with glucan synthase mutations.

\section{POSTER PRESENTATIONS}

\section{P01 \\ ANTIPROTOZOAL POTENTIAL OF PHENOLIC COMPOUNDS FROM KLEINIA ODORA}

N Al Musayeib ${ }^{1}$, R Mothana ${ }^{1}$, A El Gamal ${ }^{1}$, S Al-Massarani ${ }^{1}$, L Ames ${ }^{2}$ ${ }^{1}$ King Saud University, Riyadh, Saudi Arabia; ${ }^{2}$ Antwerp University, Antwerp, Belgium

Today, over one billion people worldwide are at risk of tropical diseases caused by parasitic protozoa. Several antiparasitic drugs have been derived directly from natural sources, such as quinine, artemisinin and amphotericin B. In this study phenolic constituents of Kleinia odora growing in Saudi Arabia were isolated and assessed for antiprotozoal activity. They were screened in vitro against erythrocytic schizonts of Plasmodium falciparum, intracellular amastigotes of Leishmania infantum, Trypanosoma cruzi and free trypomastigotes of $T$. brucei. Cytotoxic activity was determined against MRC-5 cells to assess selectivity. The compounds were identified as dihydrodehydrodiconiferyl alcohol (1), (7S,8R)-1-(3,4-dimethoxyphenyl)-2O-(2-methoxy-4-omegahydroxypropylphenyl) propane-1,3-diol (2), (7S,8S)-1-(4-hydroxy-3-methoxyphenyl)-2-O-(2-methoxy-4omegahydroxypropylphenyl) propane-1,3-diol (3) caffeic acid (4), 3,3'-dimethoxyquercetin, 7-O- $\beta$-D-glucopyranoside (5), narcissin (6) and rutin (7). In vitro antiprotozoal investigation revealed marginal antiplasmodial activity of the lignans $1-3$ against $P$. falciparum $\left(\mathrm{IC}_{50}\right.$ ranging from 8 to $10 \mu \mathrm{M})$ and mild antitrypanosomal effect by compounds 1 and 2 . Additionally and besides its activity against $\mathrm{T}$. brucei ( $\left.\mathrm{IC}_{50} 4.0 \mu \mathrm{M}\right)$, compound 5 showed antileishmanial effect comparable to standard drug miltefosine against $L$. infantum ( $\left.\mathrm{IC}_{50} 9.0 \mu \mathrm{M}\right)$.

\section{P02}

IN-VITRO ANTIVIRAL ACTION OF AMYLMETACRESOL (AMC) AND 2,4-DICHLOROBENZYL ALCOHOL (BCDA) AGAINST THE RESPIRATORY PATHOGEN PARAINFLUENZA TYPE 3

\section{A Shephard ${ }^{1,2}$, S Zybeshari ${ }^{1}$}

${ }^{1}$ Reckitt Benckiser, Slough; ${ }^{2}$ Society of Biology, London, UK

OBJECTIVES: Antibiotic resistance continues to be a global threat and is in part fuelled by their inappropriate use for primarily viral respiratory tract infections. With viral infections accounting for $80-90 \%$ of all sore throats and a wide variety of potential virus types involved, there is a need for better treatment options for this common condition.

METHODS: Parainfluenza Type 3 is a common respiratory pathogen which can cause sore throat. The ASTM international standard method E1052-11 for testing microbicides against viruses in suspension was used to evaluate the effect of AMC and DCBA in combination and a dissolved lozenge containing both AMC and DCBA against parainfluenza type 3 virus under in-vitro conditions.

RESULTS: $0.6 \mathrm{mg}$ AMC and $1.2 \mathrm{mg}$ DCBA in $5 \mathrm{ml}$ of artificial saliva exhibited a $4.18-4.56 \log _{10}$ reduction in viral titre at 1 minute. The lozenge containing $0.6 \mathrm{mg}$ of AMC and $1.2 \mathrm{mg}$ of DCBA dissolved in $5 \mathrm{ml}$ of artificial saliva exhibited a $2.43-2.68 \log _{10}$ reduction in viral titre at 1 minute. A placebo lozenge without AMC and DCBA exhibited a $0-0.43 \log _{10}$ reduction in viral titre.

CONCLUSIONS: AMC and DCBA as free active substances and when incorporated into a lozenge delivery format are able to rapidly inactivate parainfluenza virus type 3 under in-vitro conditions. AMC and DCBA have already been demonstrated to inactivate other enveloped respiratory viruses including influenza, respiratory syncytial virus and coronavirus making them a potential candidate for further clinical investigation as an alternative to antibiotics in controlling viral respiratory tract infections

\section{P03}

\section{USE OF TEMOCILLIN RESISTANCE (TEMR) AS A PHENOTYPIC DIFFUSION MARKER FOR CLASS D CARBAPENEMASES (CARB) IN CARBAPENEMASE- PRODUCING ORGANISMS (CPO)}

BM Willey ${ }^{1}$, DN Grohn ${ }^{2}$, P Rahman ${ }^{1}$, DA Boyd ${ }^{3}$, X Trimi $^{4}$, O Leung 5 , S Dunna ${ }^{4}$, P Lo ${ }^{1}$, G Ricci ${ }^{6}$, T Mazzulli ${ }^{1,4}$, SM Poutanen ${ }^{1,4}$ ${ }^{1}$ Mount Sinai Hospital/University Health Network; ${ }^{2}$ Michener Institute, Toronto, ON; ${ }^{3}$ National Microbiology Laboratory, Winnipeg, MB; ${ }^{4}$ University of Toronto, Toronto; ${ }^{5}$ University of Western Ontario, London; ${ }^{6}$ William Osler Health Sciences Centre, Brampton, ON

OBJECTIVES: Zone differences for meropenem with/without boronic or dipicolinic (DP) acid inhibitors predict class A or B Carb, respectively. But this test has no means to distinguish class D (OXA-like) CPO from non-CPO. Using ROSCO Tem tablets [MBL+KPC+OXA48 Confirm Kit (ProLab)], we evaluated the utility of TemR for predicting OXA-like Carb CPO.

METHODS: The 228 Gram-negative bacilli (mostly enterobacteria) blinded for Tem diffusion as per CLSI included 194 CPO [105 Class A: 100 KPC, 3 SME, 2 NMC; 60 class B: 53 NDM, 6 VIM, 1 IMP7; 24 class D: 13 OXA48, 6 OXA181, 3 OXA232, 1 OXA24, 1 OXA244; 5 Class B+D: 3 NDM+OXA181, 2 NDM+OXA232) and 34 non-CPO (ESBL, ampC-hyperproducers, ompC/F or ompK35/K36 mutants). After plating twice to MacConkey agar with ertapenem discs in the main inoculum to maintain selective pressure, growth closest to the disc was used to make 0.5 MacFarland suspensions for inoculation to Oxoid Mueller-Hinton Plus agar with Tem tablets. After $18 \mathrm{~h}$ incubation at $35^{\circ} \mathrm{C}$, consensus zones compiled from 5 independent reads were determined. Results were then correlated with $\mathrm{CPO}$ genotypes and a breakpoint to maximize specificity (Sp) without loss of sensitivity $(\mathrm{Sn})$ for $\mathrm{CPO}$ was determined.

RESULTS: A breakpoint of $<12 \mathrm{~mm}$ for TemR was chosen as it correlated with $100 \%$ (95\% CI: 88-100) Sp as 34/34 non-CPO had zones $>11 \mathrm{~mm}$ (range: $12-33 \mathrm{~mm}$ ). TemR was detected in $24 / 24$ class $\mathrm{D}, 5 / 5$ with class $\mathrm{B}+\mathrm{D}$ (OXA181/232 masked the DP+ result expected for NDM in 4/5 strains), 46/60 class B (TemR included 6/6 VIM, 2 of which were DP-neg; range for 14 NDM not TemR: $12-22 \mathrm{~mm}$ ), and 1/105 class A 
(1 SME+ S. marcescens with zone $9 \mathrm{~mm}$; range for 104 not TemR: $14-29 \mathrm{~mm})$. Sn by this criterion for CPO by Carb class was: D (100\%; $95 \%$ CI: 86-100), B (77\%; 95\% CI: 67-87), and A (1\%; 95\% CI: <0.01-5.7). CONCLUSIONS: These data indicate that the simple, inexpensive ROSCO Tem diffusion test used with a zone of $<12 \mathrm{~mm}$ to indicate TemR serves as a highly sensitive marker for the elusive class D OXA-type CPO, as $100 \%$ had Tem zones of $9 \mathrm{~mm}$ (no zone). This test was also highly specific for $\mathrm{CPO}$ as $100 \%$ non-CPO had Tem zones $>11 \mathrm{~mm}$. Tem also enhanced phenotypic detection of $\mathrm{CPO}$ when class $\mathrm{B}$ CPO were poorly expressed and where isolates were not typically inhibited by DP as in two distinct VIM CPO in this study.

\section{P04}

\section{WITHDRAWN}

\section{P05}

\section{NOVEL ISOSTEVIOL ISOLATED FROM PITTOSPORUM TETRASPERMUM EXHIBITED ANTIMICROBIAL, ANTIBIOFILM AND ANTICANCER ACTIVITIES}

\section{VA Mariadhas, NA Al-Dhabi}

Department of Botany and Microbiology, Addiriyah Chair for Environmental Studies, College of Science, King Saud University, Riyadh, Saudi Arabia

Drugs derived from medicinal plants as green medicine in recent years has attracted many researchers because of its widespread applications in curing infectious diseases, compared with costly synthetic drugs that have adverse effects. Aim of this study was to investigate the in vitro antimicrobial, antibiofilm and anticancer properties of isosteviol isolated from endangered medicinal plant Pittosporum tetraspermum. Pure compound was obtained and characterized by column chromatography followed by ${ }^{1} \mathrm{H}$ NMR, ${ }^{13} \mathrm{C}$ NMR, IR and Mass spectral analysis. The antibacterial and antifungal activities of the compound were assessed by the broth micro dilution method. Anticancer study was evaluated by following MTT assay. Primary screening revealed that the organic extract of the compound inhibited the growth of microbial pathogens. Further, column purification and spectrocopical analysis guided to identify novel isosteviol from the crude ethyl acetate extract. The compound exhibited significant activity against bacteria such as Staphylococcus epidermidis (125 $\mu \mathrm{g} / \mathrm{mL})$, Staphylococcus aureus $(125 \mu \mathrm{g} / \mathrm{mL})$ and Klebsiella pneumoniae $(62.5 \mu \mathrm{g} / \mathrm{mL})$. The MIC of the compound against Candida albicans, Aspergillus niger and Trichophyton mentagrophytes were 62.5, 125 and $500 \mu \mathrm{g} / \mathrm{mL}$ respectively. The compound showed comparatively better antibiofilm activity against the uropathogens such as E. coli, S. typhi and P. aeroginosa. Furthermore, it exhibited good anticancer properties against Vero and MCF7 cell lines. Novel isosteviol would be useful to reduce the infectious diseases caused by pathogenic bacteria and fungi or slowing the progress of various oxidative stress-related diseases.

\section{P06}

CYP2C19*17 GENETIC POLYMORPHISM AND THERAPEUTIC CHALLENGES IN AN ALLOGENEIC STEM CELL TRANSPLANT RECIPIENT WITH INVASIVE PULMONARY ASPERGILLOSIS

\section{Abidi ${ }^{1}$, A D'Souza ${ }^{1}$, H Parmeswaran ${ }^{1}$, K Kuppalli $^{2}$}

${ }^{1}$ Medical College of Wisconsin, Milwaukee, WI; ${ }^{2}$ Loyola University Medical Center, Chicago, IL, USA

BACKGROUND: A 48-year-old male, allogeneic hematopoietic stem cell transplant recipient with extensive graft versus host disease was admitted with pulmonary aspergillosis 6 months after transplant. Chest computerized tomography (CT) scan showed bilateral lung opacities and he required intubation. Antimicrobials included intravenous cefepime, azithromycin and voriconazole. Bronchoalveolar lavage (BAL) fluid cultures grew $10,000 \mathrm{cfu} / \mathrm{mL}$ Pseudomonas aeruginosa and 1,000 cfu/mL Aspergillus fumigatus. Nucleic acid amplification testing was positive for Mycoplasma pneumoniae. He improved and was switched to oral voriconazole. A week later, his respiratory status worsened and Chest CT showed worsening opacities. Voriconazole serum levels were undetectable twice. We suspected that he might be an ultra metabolizer of voriconazole.
METHODS: Cytochrome assay was requested. The methodology employed was DNA analysis of the cytochrome P450 2C19 gene including the alleles for poor metabolizers *1, $* 2, * 3 * 4, * 5, * 6, * 7$ and $* 8$ as well as the ultra-metabolizer alleles $* 17$. We added micafungin and one week later imaging showed improvement. CYP2C19 genotype testing was positive for the *17 translocation indicating he was an ultra metabolizer for voriconazole. He was discharged home on micafungin with plans to switch to liposomal amphotericin B if there was any worsening.

RESULTS: Genetic polymorphism of CYP2C19 modulates enzyme activity and significantly different plasma concentrations are observed despite identical dosing schedules. Studies reveal polymorphisms to contribute to pharmacokinetic variability. Individuals may be categorized as ultra rapid metabolizers (UM) (CYP2C19*1/*17 CYP2C19*17/*17); extensive metabolizers (EM) (CYP2C19*1/*1); intermediate metabolizer (IM) $(\mathrm{CYP} 2 \mathrm{C} 19 * 1 / * 2$ and CYP2C19*1/*3) and poor metabolizers (PM) (CYP2c19*2/*2, CYP2C19*2/*3 and CYP2C19*3/*3)(5). This study highlighted that voriconazole is influenced by CYP2C19 polymorphism and gene based adjusted dosing is important to achieve therapeutic drug levels.

CONCLUSIONS: Clinicians should keep CYP2C19 polymorphisms in the differential when evaluating very low voriconazole levels for treatment of invasive fungal infections.

\section{P07}

NANOPARTICLES TO TARGET HIV RESERVOIRS

C Lavigne $^{1}$, E Berger ${ }^{1}$, S Stals ${ }^{1}$, T Read ${ }^{2}$, M-A Langlois ${ }^{2}$,

D Gonçalves $^{3}$, D Girard ${ }^{3}$, D Breznan ${ }^{4}$

${ }^{1}$ Public Health Agency of Canada, Winnipeg, MB; ${ }^{2}$ University of Ottawa, Ottawa, ON; ${ }^{3}$ INRS-Institut Armand-Frappier, Laval, QC; ${ }^{4}$ Health Canada, Ottawa, ON

BACKGROUND: Although the current antiretroviral drugs (ARVs) can effectively inhibit replication of HIV-1 in infected patients, the virus is able to persist in cellular and anatomical viral reservoirs leading to latent re-infection and development of drug resistance. Macrophages constitute one of the most important viral reservoirs and are able to transport HIV into other reservoir sites such as the lymphoid organs and the central nervous system (CNS). ARVs hardly penetrate into the CNS and macrophages therefore, it is highly critical to improve drug delivery in these compartments.

OBJECTIVE: Here we evaluated the lipid-based nanosystem Neutraplex $\mathrm{Nx})$ for the transport of therapeutic molecules into macrophages and in the brain to target HIV viral reservoirs.

METHODS: We have determined the cytotoxicity profile of the Neutraplex nanosystem using different cell models (TZM-bl, THP-1, $\mathrm{Be}(2)-\mathrm{M} 17$ ) and various cytotoxicity assays. Cellular uptake was evaluated by confocal microscopy. In addition, $\mathrm{Nx}$ capability to cross the blood-brain barrier (BBB) was investigated using the immortalized adult rat brain microvascular endothelial cell model (SV-ARBEC) and effect on inflammatory response was evaluated using an in vitro neutrophil apoptosis assay. RESULTS: Nx nanoparticles (NP) were not found cytotoxic up to the highest dose tested, $35 \mu \mathrm{g} / \mathrm{mL}$. Confocal microscopy studies showed that Nx NPs are rapidly and efficiently taken up by THP-1 macrophages and were still abundantly found in the cells $48 \mathrm{~h}$ following exposure, at doses as low as $4 \mu \mathrm{g} / \mathrm{mL}$. In addition, Nx NPs were able to transmigrate the endothelial cell monolayer and were not found cytotoxic for neuronal cells, suggesting that they have the capability to deliver drugs through the BBB without affecting neuronal viability. Finally, Nx NPs did not modulate apoptosis of polymorphonuclear neutrophils indicating their low interference with inflammatory response.

CONCLUSION: Altogether these results indicate that the Neutraplex nanocarrier shows potential as a delivery strategy aiming to target HIV in cellular and anatomical viral reservoirs. 
P08

INCREASED RECOVERY AND DETECTION OF PATHOGENS USING COPAN'S WASPLAB ${ }^{\text {TM }}$ TOTAL LABORATORY AUTOMATION (TLA)

M McConnell ${ }^{1}$, C Main ${ }^{1,2}$, W McCaffrey ${ }^{1}$, D Skorchid ${ }^{1}$, D Yamamura ${ }^{1,2}$ ${ }^{1}$ Hamilton Health Sciences, Hamilton Regional Microbiology

Laboratory; ${ }^{2}$ Department of Pathology and Molecular Medicine, McMaster University, Hamilton, ON

BACKGROUND: The objective of this study was to observe the effects of using Copan's WASPLab ${ }^{\mathrm{TM}}$ Total Laboratory Automation (TLA) on the recovery and detection of pathogens from clinical specimens.

METHODS: A wide range of specimens were processed on TLA using a customized automation platform (inoculation, incubation in Smart Incubators, and digital image reporting). Consecutive specimens were processed in real-time simultaneously and blinded on TLA and manually according to standard operating procedures. Analytical and post-analytical processes followed incubation of plates in the Smart Incubators and manually in off-line incubators. Identification of pathogens was achieved using a combination of mass spectrometry, Vitek2, chromogenic media, and standard biochemical tests.

RESULTS: A total of 874 specimens were evaluated in parallel comparing TLA with routine methods. For urine cultures $(n=264)$ using Orientation CHROMagar, $16 \%(n=43)$ of specimens had positive cultures $\left(\geq 10 \times 10^{6}\right.$ CFU/L) using TLA vs. no growth with manual processing. 18/43 (42\%) grew significant pathogens. Faecal specimens $(n=58)$ using standard enteric media, 19\% ( $\mathrm{n}=11)$ were screened positive using TLA vs. manual processing, however all were non-pathogenic (H. alvei, M. morganii, $P$. aeruginosa, and C. amalonaticus). For sterile fluids ( $\mathrm{n}=35)$, additional pathogens (E. coli and Enterococcus faecalis) were isolated from abscess fluid recovered from Thio broth processed on TLA. For vaginal/rectal specimens $(n=25)$, one additional pathogen (GBS) was detected using LIM broth enrichment and chromogenic agar sub-culture by TLA vs. manual processing. Surveillance specimens [MRSA $(n=251), \operatorname{VRE}(n=220)$, and ESBL $(n=21)]$ were tested using various chromogenic and selective agars. A $4 \%$ increase $(n=19)$ of specimens had positive cultures using TLA vs. manual processing. CONCLUSIONS: Using Copan's TLA resulted in an increase in pathogen recovery when compared to manual processes. This increase can be attributed to the standardized inoculation of specimens, consistent streaking and isolation of colonies when compared to manual processes. With the immediate incubation of media following automated processing, the uniform temperatures and atmospheric conditions maintained in the Smart Incubators creates a favorable environment conducive to bacterial growth and detection.

\section{P09}

USEFULNESS OF PREVIOUS METHICILLIN-RESISTANT STAPHYLOCOCCUS AUREUS SCREENING RESULTS IN GUIDING EMPIRIC THERAPY FOR S. AUREUS BACTEREMIA

A Bai $^{1}$, L Burry ${ }^{2,3}$, A Showler ${ }^{4}$, M Steinberg ${ }^{2}$, D Ricciuto ${ }^{4,5,6}$,

T Fernandes ${ }^{7}$, A Chiu', S Raybardhan ${ }^{8}$, G Tomlinson ${ }^{4,9}$, C Bell ${ }^{2,4,10,}$ A Morris $2,4,9,10$

${ }^{1}$ University of Ottawa, Ottawa; ${ }^{2}$ Mount Sinai Hospital; ${ }^{3}$ Leslie Dan Faculty of Pharmacy; ${ }^{4}$ University of Toronto, Toronto; ${ }^{5}$ Lakeridge Health, Oshawa; ${ }^{6}$ Queen's University, Kingston; ${ }^{7}$ Trillium Health Partners, Mississauga; ${ }^{8}$ North York General Hospital; ${ }^{9}$ University Health Network; ${ }^{10}$ Institute for Clinical Evaluative Sciences, Toronto, ON

OBJECTIVES: Staphylococcus aureus bacteremia (SAB) is an important infection. Methicillin-resistant Staphylococcus aureus (MRSA) screening is performed in hospitalized patients to guide infectious disease control practices. We conducted a retrospective cohort study to assess the usefulness of past MRSA screening in guiding empiric antibiotic therapy for SAB.

METHODS: A retrospective cohort study examined consecutive patients with confirmed SAB and prior MRSA swab screening from 6 academic and community hospitals between 2007 and 2010. Diagnostic test properties were calculated for MRSA swab screening in predicting methicillin susceptibility of SAB.
RESULTS: In the study, 799 patients had MRSA screening swabs prior to SAB. Of the 799 patients, 95 (12\%) patients had a positive and 704 (88\%) had a negative prior MRSA screening swab. There were 150 (19\%) patients with MRSA bacteremia. Overall, prior MRSA screening swab had a positive likelihood ratio of 33 (CI: 18-60) and negative likelihood ratio of 0.45 (CI: 0.37-0.54). Diagnostic accuracy differed depending on mode of acquisition (i.e. community-acquired, nosocomial, or healthcare-associated infection) $(\mathrm{P}<0.0001)$ and hospital $(\mathrm{P}=0.0002)$. Mathematical modeling of this data demonstrates that the post-test probability of MRSA for a negative MRSA screening result is close to or below $10 \%$ when MRSA prevalence in SAB is less than $25 \%$.

CONCLUSIONS: MRSA screening swabs can be helpful in guiding empiric antibiotic therapy for $\mathrm{SAB}$ in areas with moderate MRSA prevalence.

\section{P10}

\section{INADEQUATE SYMPTOM DOCUMENTATION OF URINARY TRACT INFECTION IN THE MEDICAL CHART}

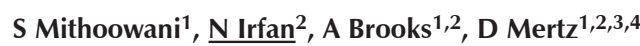

${ }^{1}$ Department of Medicine, McMaster University; ${ }^{2}$ Hamilton Health Sciences; ${ }^{3}$ Department of Clinical Epidemiology and Biostatistics, McMaster University; ${ }^{4}$ Michael G DeGroote Institute for Infectious Diseases Research, McMaster University, Hamilton, ON

OBJECTIVES: Small studies suggest clinicians inconsistently document symptoms in the medical chart for patients suspected of having urinary tract infection (UTI). Our goal was to prospectively evaluate the completeness of clinician documentation, and to compare it with data obtained from a prospective interview with the medical team caring for the patient. METHODS: Consecutive inpatients at two academic, tertiary care hospitals with positive urine cultures from January 30 to April 17, 2012 were included. Urinary symptoms were documented prospectively by interviewing physicians and nurses caring for the patient and compared to an independent retrospective review of the patient's chart upon discharge.

RESULTS: During the study period, 341 patients were eligible and 312 were included for analysis ( 29 were excluded due to inability to interview the medical team caring for the patient). Amongst these patients, $174 / 312$ (56\%) had symptomatic UTI when combining information from the interviews and charts, which we considered our gold standard (Table).

\begin{tabular}{lccc} 
Symptom & $\begin{array}{c}\text { Interview and/ } \\
\text { or chart }\end{array}$ & Interview $\mathbf{n}(\%)$ & Chart review n (\%) \\
\hline Any symptom & 174 & $150(86)$ & $134(77)$ \\
Delirium & 84 & $61(73)$ & $59(70)$ \\
Fever & 65 & $43(66)$ & $46(71)$ \\
Frequency & 47 & $33(70)$ & $27(57)$ \\
Dysuria & 39 & $27(69)$ & $24(62)$ \\
Hematuria & 16 & $8(50)$ & $8(50)$ \\
Flank pain & 9 & $6(67)$ & $4(44)$ \\
Other symptoms & 17 & $6(35)$ & $15(88)$ \\
\hline
\end{tabular}

CONCLUSIONS: Due to insufficient documentation of symptoms, a significant number of patients with UTI would be misclassified as asymptomatic bacteriuria with retrospective chart review alone. Infection surveillance or audits for asymptomatic bacteriuria should incorporate prospective data collection from health care providers in addition to chart review to avoid underestimating the number of symptomatic patients. 
P11

ALL-ORAL 12-WEEK COMBINATION TREATMENT WITH DACLATASVIR (DCV) AND SOFOSBUVIR (SOF) IN PATIENTS INFECTED WITH HCV GENOTYPE (GT) 3: ALLY-3 PHASE 3 STUDY

DR Nelson ${ }^{1}$, JN Cooper ${ }^{2}$, JP Lalezari ${ }^{3}$, E Lawitz ${ }^{4}$, PJ Pockros ${ }^{5}$, N Gitlin ${ }^{6}$, BF Freilich $^{7}$, ZH Younes ${ }^{8}$, W Harlan ${ }^{9}$, R Ghalib ${ }^{10}$, G Oguchi $^{11}$, P Thuluvath ${ }^{12}$, G Ortiz-Lasanta ${ }^{13}, M_{\text {Rabinovitz }}{ }^{14}$, D Bernstein ${ }^{15}$, M Bennett ${ }^{16}$, T Hawkins ${ }^{17}$, N Ravendhran ${ }^{18}$, AM Sheikh ${ }^{19}$,

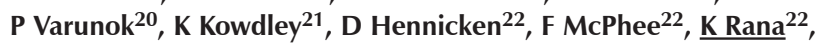
EA Hughes 22

${ }^{1}$ University of Florida, Gainesville, FL; ${ }^{2}$ Inova Fairfax Hospital, Falls Church, VA; ${ }^{3}$ Quest Clinical Research, San Francisco, CA; ${ }^{4}$ Texas Liver Institute, University of Texas Health Science Center, San Antonio, TX; ${ }^{5}$ Scripps Clinic, La Jolla, CA; ${ }^{6}$ Atlanta Gastroenterology Associates, Atlanta, GA; ${ }^{7}$ Kansas City Research Institute, Kansas City, MO; ${ }^{8}$ Gastro One, Germantown, TN; ${ }^{9}$ Asheville Gastroenterology Associates, Asheville, NC; ${ }^{10}$ Texas Clinical Research Institute, Arlington, TX; ${ }^{11}$ Midland Florida Clinical Research Center, DeLand, FL; ${ }^{12}$ Mercy Medical Center, Baltimore, MD, USA; ${ }^{13}$ Fundación de Investigación de Diego, Santurce, Puerto Rico; ${ }^{14}$ University of Pittsburgh, Pittsburgh, PA; ${ }^{15}$ Hofstra North Shore-Long Island Jewish School of Medicine, Manhasset, NY; ${ }^{16}$ Medical Associates Research Group, San Diego, CA; ${ }^{17}$ Southwest CARE Center, Santa Fe, NM; ${ }^{18}$ Digestive Disease Associates, Baltimore, MD; ${ }^{19}$ Gastrointestinal Specialists of Georgia, Marietta, $\mathrm{GA} ;{ }^{20}$ Premier Medical Group of Hudson Valley, Poughkeepsie, NY; ${ }^{21}$ Swedish Medical Center, Seattle, WA; ${ }^{22}$ Bristol-Myers Squibb Research and Development, Princeton, NJ, USA

BACKGROUND: Options for treating HCV GT 3 infection are limited in both treatment-naive and -experienced patients; the only currently available all-oral regimen requires 24 -week treatment that includes ribavirin (RBV). Newer combinations without RBV are being studied to shorten treatment duration. The efficacy and safety of the combination of DCV (potent, pangenotypic NS5A inhibitor) and SOF (NS5B polymerase inhibitor) for 12 weeks were evaluated in patients chronically infected with GT 3.

METHODS: Two cohorts consisting of treatment-naive or -experienced (prior treatment failures, including prior $\mathrm{SOF}$ - or alisporivir-treated) patients received open-label DCV $60 \mathrm{mg}$ + SOF $400 \mathrm{mg}$ QD for 12 weeks. Efficacy (sustained virologic response at posttreatment Week 4 [SVR4]) and safety outcomes are reported. SVR12, the primary endpoint, will be available for presentation.

RESULTS: 152 patients were treated: $101(66 \%)$ treatment-naive and 51 (34\%) treatment-experienced; $21 \%$ were cirrhotic, $61 \%$ non-CC IL28B genotype, $71 \% \mathrm{HCV}$ RNA $\geq 800 \mathrm{~K} \mathrm{IU} / \mathrm{mL}$. Baseline characteristics were comparable between the 2 cohorts except for a higher proportion of cirrhotic patients in the treatment-experienced cohort. Overall, $91 \%$ and $86 \%$ of treatment-naive and -experienced patients, respectively, achieved SVR4; SVR4 rates were $94 \%$ for patients with a METAVIR category of F0-F3 and 70\% for those with F4. One patient had detectable HCV RNA at the end of treatment and 15 had relapse posttreatment (mostly cirrhotic patients). One serious adverse event was reported on-treatment: a gastrointestinal bleed considered not related to study treatment. There were few Grade 3/4 laboratory abnormalities - observed only for platelets $(n=2)$, lymphocytes $(n=1)$, international normalized ratio (INR; $n=2)$, and lipase $(n=3)$; these did not lead to treatment discontinuations. The most frequent adverse events (all grades) were headache (20\%), fatigue (18\%), nausea $(12 \%)$, and diarrhea $(9 \%)$.

CONCLUSION: This all-oral, RBV-free, 12-week combination of DCV+SOF in HCV GT 3 infection achieved SVR4 rates of $91 \%$ and $86 \%$ in treatment-naive and -experienced patients, respectively, with higher rates in those with METAVIR FO-F3. This combination was safe and well tolerated.

\section{P12}

ALL-ORAL, FIXED-DOSE COMBINATION THERAPY WITH DACLATASVIR/ASUNAPREVIR/BMS-791325 FOR NONCIRRHOTIC PATIENTS WITH CHRONIC HCV GENOTYPE 1 INFECTION: UNITY-1 PHASE 3 SVR12 RESULTS

F Poordad ${ }^{1}$, W Sievert ${ }^{2}$, L Mollison ${ }^{3}$, N Brau ${ }^{4}$, J Levin ${ }^{5}$, S Sepe $^{6}$, S Lee ${ }^{7}$, N Boyer ${ }^{8}$, J-P Bronowicki ${ }^{9}$, IM Jacobson ${ }^{10}$, N Boparai ${ }^{11}$, EA Hughes ${ }^{11}$, ES Swenson ${ }^{12}$, PD Yin ${ }^{12}$

${ }^{1}$ Texas Liver Institute, University of Texas Health Science Center, San Antonio, TX, USA; ${ }^{2}$ Monash Health and Monash University, Melbourne; ${ }^{3}$ Fremantle Hospital, Hepatitis Services, Fremantle, Australia; ${ }^{4}$ Bronx Veterans Affairs Medical Center, New York, NY; ${ }^{5}$ Dean Foundation, Madison, WI, USA; ${ }^{6}$ University

Gastroenterology, Providence, RI, USA; ${ }^{7}$ University of Calgary, Calgary, AB; ${ }^{8}$ Service d'Hépatologie, Hôpital Beaujon, Clichy, France; ${ }^{9}$ Centre Hospitalier Universitaire de Nancy, Université de Lorraine, Vandoeuvre les Nancy, France; ${ }^{10}$ Weill Cornell Medical College, New York, NY; ${ }^{11}$ Bristol-Myers Squibb, Princeton, NJ;

${ }^{12}$ Bristol-Myers Squibb, Wallingford, CT, USA

INTRODUCTION: The all-oral combination of daclatasvir (DCV; pangenotypic NS5A inhibitor), asunaprevir (ASV; NS3 protease inhibitor), and BMS-791325 (non-nucleoside NS5B inhibitor) - DCV 3DAA regimen - was evaluated without ribavirin in HCV genotype (GT) 1 -infected treatment-naive and -experienced patients without cirrhosis in a Phase 3, open-label, international clinical trial.

METHODS: Patients received a fixed-dose combination (FDC) of DCV $30 \mathrm{mg}$, ASV $200 \mathrm{mg}$, and BMS-791325 $75 \mathrm{mg}$ twice daily for 12 weeks. SVR12 rates in the treatment-naive and -experienced cohorts were evaluated separately as key efficacy outcomes.

RESULTS: SVR12 was achieved by $92 \%$ of treatment-naive patients (Table). Among treatment-experienced patients, $89 \%$ achieved SVR12. Virologic failure occurred in $34(8 \%)$ patients overall. Baseline characteristics were comparable between the treatment-naive $(\mathrm{N}=312)$ and treatment-experienced $(\mathrm{N}=103)$ cohorts. Overall, patients were $58 \%$ male and 26\% IL28B (rs1297860) CC genotype; 73\% were infected with GT 1a and $27 \%$ with GT 1b. One death reported posttreatment was considered not related to study treatment. There were 7 serious adverse events, all considered unrelated to study treatment, and $3(<1 \%)$ adverse events leading to treatment discontinuation. The most common adverse events (in $>10 \%$ of patients) were headache, fatigue, diarrhea, and nausea.

CONCLUSIONS: In this Phase 3 study of 415 patients, 12 weeks of alloral treatment with DCV/ASV/BMS-791325 FDC achieved high SVR12 rates in patients with chronic HCV GT 1 infection and was well tolerated. These findings demonstrate the potent antiviral activity, safety, and tolerability of the DCV 3DAA regimen in treatment-naive and treatmentexperienced patients without cirrhosis.

\begin{tabular}{lcc}
\hline & Treatment-naive & Treatment-experienced \\
\hline Virologic outcomes, $\mathrm{n}(\%)$ & $\mathrm{DCV}$ 3DAA (N=312) & DCV 3DAA (N=103) \\
SVR12 & $287(92)$ & $92(89)$ \\
Posttreatment relapse & $15(5)$ & $6(6)$ \\
On-treatment failure & $9(3)$ & $4(4)$ \\
Missing data & $1(<1)$ & $1(<1)$ \\
\hline
\end{tabular}


P13

ALL-ORAL FIXED-DOSE COMBINATION THERAPY WITH DACLATASVIR/ASUNAPREVIR/BMS-791325, \pm RIBAVIRIN, FOR PATIENTS WITH CHRONIC HCV GENOTYPE 1 INFECTION AND COMPENSATED CIRRHOSIS: UNITY-2 PHASE 3 SVR12 RESULTS

AJ Muir ${ }^{1}$, F Poordad ${ }^{2}$, J Lalezari ${ }^{3}$, GJ Everson ${ }^{4}$, GJ Dore ${ }^{5}$, P Kwo ${ }^{6}$, C Hézode ${ }^{7}$, PJ Pockros ${ }^{8}$, A Tran ${ }^{9}$, A Ramji ${ }^{10}$, R Yang ${ }^{11}$, EA Hughes ${ }^{11}$, ES Swenson ${ }^{12}$, PD Yin ${ }^{12}$

${ }^{1}$ Duke Clinical Research Institute, Duke University School of Medicine, Durham, NC; ${ }^{2}$ Texas Liver Institute, University of Texas Health Science Center, San Antonio, TX; ${ }^{3}$ Quest Clinical Research, San Francisco, CA; ${ }^{4}$ University of Colorado School of Medicine, Denver, CO, USA; ${ }^{5}$ Kirby Institute, UNSW Australia, Sydney, NSW, Australia; ${ }^{6}$ Indiana University School of Medicine, Indianapolis, IN, USA; ${ }^{7}$ Hôpital Henri Mondor, Université Paris-Est, Créteil, France; ${ }^{8}$ Scripps Clinic, La Jolla, CA, USA; ${ }^{9}$ Centre Hospitalier Universitaire de Nice, Nice, France; ${ }^{10}$ University of British Columbia, Vancouver, $\mathrm{BC} ;{ }^{11}$ Bristol-Myers Squibb, Princeton, NJ; ${ }^{12}$ Bristol-Myers Squibb, Wallingford, CT, USA

INTRODUCTION: The all-oral combination of daclatasvir (DCV; pangenotypic NS5A inhibitor), asunaprevir (ASV; NS3 protease inhibitor), and BMS-791325 ('325; non-nucleoside NS5B inhibitor) - DCV 3DAA regimen - was studied with and without ribavirin (RBV) in treatment-naive and treatment-experienced patients with HCV genotype (GT) 1 infection and compensated cirrhosis in a Phase 3 , international clinical trial.

METHODS: Patients were randomly assigned to receive a fixed-dose combination (FDC) of DCV $30 \mathrm{mg}$, ASV $200 \mathrm{mg}$, and '325 $75 \mathrm{mg}$, with blinded RBV or placebo, twice-daily for 12 weeks. SVR12 rates in the treatment-naive and experienced cohorts were evaluated separately as key efficacy outcomes.

RESULTS: SVR12 results in treatment-naive and -experienced cirrhotic patients are in the table below. Virologic failure was observed in $13(6 \%)$ patients. Baseline characteristics were comparable between treatmentnaive $(\mathrm{N}=112)$ and treatment-experienced $(\mathrm{N}=90)$ groups. Overall, patients were $66 \%$ male and 27\% IL28B (rs1297860) CC genotype; 74\% of patients had GT1a infection and $26 \%$ had GT1b. There were 3 serious adverse events (SAEs) considered related to treatment, 1 AE leading to 3DAA discontinuation, and no deaths. The most frequent AEs (>10\% of patients) were fatigue, headache, nausea, diarrhea, insomnia and pruritus. Hemoglobin $<9 \mathrm{~g} / \mathrm{dL}$ on treatment was observed in $5 \%$ of patients in the RBV-containing cohorts but in no patients in the RBV-free cohorts.

CONCLUSIONS: Twelve weeks of all-oral treatment with DCV/ASV/ BMS-791325 FDC, with or without ribavirin, achieved high rates of SVR12 in 202 cirrhotic patients with GT1 infection. These results demonstrate the potent antiviral activity, tolerability and safety of the DCV 3DAA regimen in patients with compensated cirrhosis.

\begin{tabular}{|c|c|c|c|c|}
\hline \multirow[b]{2}{*}{$\begin{array}{l}\text { Virologic out- } \\
\text { comes, } \mathrm{n}(\%)\end{array}$} & \multicolumn{2}{|c|}{$\begin{array}{l}\text { Treatment-naive } \\
\text { cirrhotics }^{*}\end{array}$} & \multicolumn{2}{|c|}{$\begin{array}{c}\text { Treatment-experienced } \\
\text { cirrhotics }^{*}\end{array}$} \\
\hline & $\begin{array}{l}\text { DCV 3DAA } \\
(\mathrm{N}=57)\end{array}$ & $\begin{array}{l}\mathrm{DCV} \text { 3DAA + } \\
\mathrm{RBV}(\mathrm{N}=55)\end{array}$ & $\begin{array}{l}\text { DCV 3DAA } \\
(\mathrm{N}=45)\end{array}$ & $\begin{array}{c}\text { DCV 3DAA } \\
+ \text { RBV } \\
(\mathrm{N}=45)\end{array}$ \\
\hline SVR 12 & $53(93)$ & $54(98)$ & $39(87)$ & $42(93)$ \\
\hline $\begin{array}{l}\text { Posttreatment } \\
\text { relapse }\end{array}$ & $4(7)$ & 0 & $5(11)$ & $1(2)$ \\
\hline $\begin{array}{l}\text { On-treatment fail- } \\
\text { ure }\end{array}$ & 0 & 0 & $1(2)$ & $2(4)$ \\
\hline Missing data & 0 & $1(2)$ & 0 & 0 \\
\hline
\end{tabular}

Cirrhosis was determined by one of the following: (1) liver biopsy with Metavir F4 or equivalent, (2) Fibroscan $>14.6 \mathrm{kPa}$ within 12 months prior to screening, or (3) Fibrotest $\geq 0.75$ and AST/platelet ratio index $>2$ at screening.

\section{P14}

RECURRENT MICAFUNGIN-RESISTANT CANDIDA GLABRATA CANDIDEMIA WITH PROLONGED MICAFUNGIN TREATMENT FOR MULTIPLE INTRAABDOMINAL ABSCESSES: A CASE REPORT

HY Zhou $^{1,2}$, S Shokoples ${ }^{1,2}$, B Jansen ${ }^{1,2}$, J Fuller ${ }^{1,2}$

${ }^{1}$ Alberta Health Service; ${ }^{2}$ University of Alberta, Edmonton, AB

BACKGROUND: Candida glabrata (CG) is the second leading cause of candidemia in North America. The reports of invasive infections caused by echinocandin-resistant CG are increasing, usually in immunocompromised patients receiving prolonged echinocandin treatment.

CASE: A 45-year-old female patient with complicated medical and surgical history was admitted for multiple intra-abdominal abscesses. The medical history included Addison's disease on long term glucocorticoid replacement therapy, poorly controlled type II diabetes mellitus, severe idiopathic pancreatitis complicated by pseudocyst requiring prolonged drainage, and persistent enterocutaneous fistula that failed to close despite multiple surgical interventions. Micafungin (MICA)-susceptible (S) CG was isolated from a retroperitoneal abscess and the patient was treated with MICA for 42 days. Two days after MICA was discontinued, CG was isolated from blood cultures and MICA therapy was used for another 13 days until susceptibility results detected MICA-resistant (R) and the patient was switched to fluconazole for 14 days. Six months later, CG was cultured from Jackson-Pratt drainage of re-progressing intraabdominal abscesses, which was treated with MICA for 14 days. On day 10 of therapy, MICA R CG was again isolated from blood culture. DNA sequencing of the FSK1 and FSK2 glucan synthase loci showed mutations associated with echinocandin resistance. Patient was deceased before the susceptibility result was available.

CONCLUSION: We describe a case of recurrent MICA-R CG candidemia with prolonged MICA treatment for multiple intra-abdominal abscesses. Echinocandin resistance in Candida is uncommon but most often associated with CG and recent echinocandin exposure. Glucan synthase mutations confer high MICs, likely due to the haploid CG chromosome, and strongly correlate with clinical failure.

\section{P15}

ACTIVE ENVIRONMENTAL SURVEILLANCE OF LYME DISEASE IN BRITISH COLUMBIA (2013 AND 2014)

M-K Lee ${ }^{1}$, K Fernando', S Man'1, K Jensen', B Kahnamelli', D Tam', T Dhanoa ${ }^{1}$, Q Wong1, P Tang1,2, D Patrick 2,3, S Mak ${ }^{3}$, A Hojgaard ${ }^{4}$, B Henry ${ }^{2,5}$, M Morshed ${ }^{1,2}$

${ }^{1}$ BC Public Health Microbiology and Reference Laboratory; ${ }^{2}$ University of British Columbia; ${ }^{3}$ BC Centre for Disease Control, Vancouver, $\mathrm{BC} ;{ }^{4}$ Centers for Disease Control and Prevention, Fort Collins, CO, USA; ${ }^{5}$ BC Ministry of Health, Vancouver, BC OBJECTIVES: Lyme disease cases are increasing in many parts of Canada. However, it remains low in British Columbia. In response to public concerns regarding the low positivity rate, we conducted an active field surveillance for two years (2013-2014) to look at the prevalence of Borrelia burgdorferi in the vector (Ixodes pacificus ticks) and host (Peromyscus maniculatus mice) populations.

METHODS: Mice trapped from 11 different sites were tested by Immunofluorescence Antibody Test (IFA) and Western Blot (WB) to determine the presence of $B$. burgdorferi antibodies. Ticks were pooled from trapped mice (up to 5 ticks from each mouse per pool) for DNA extraction and real-time PCR, which targeted the 23S rRNA gene of Borrelia spp. and ospA gene of B. burgdorferi. Two organs (brain and bladder) from each mouse were subjected to the same molecular tests. A subset of tick DNA extracts $(n=96)$ were also sent to CDC Fort Collins where multiplex PCR targeting fliD and gB31genes for B. burgdorferi was performed to check for concordance between results from the two labs.

RESULTS: A total of 885 ticks at different developmental stages were retrieved from 483 mice. Serologically, 51 mice were confirmed to have antibodies against B. burgdorferi. For molecular testing, 5 tick pools were positive for B. burgdorferi but all mouse organs tested were negative. Of the 
positive tick pools, the corresponding mice were also serologically positive. The subset of ticks tested by the two laboratories had identical results. CONCLUSIONS: The positivity rate of B. burgdorferi in the British Columbia Ixodes tick population remained low and was found to be $0.6 \%$. The exposure rate in the mouse population to B. burgdorferi was determined to be $11 \%$. The low incidence in vector and low infestation rate in host population may explain the continued low incidence of Lyme disease in British Columbia.

\section{P16}

INVESTIGATING THE POTENTIAL ROLE OF BACTERIAL INFECTION IN GRANULOMATOSIS WITH POLYANGIITIS (GPA)

R Fattouh $^{1}$, BM Willey ${ }^{2}$, JV Kus ${ }^{1,3}$, T Mazzulli1,2,3

${ }^{1}$ University of Toronto; ${ }^{2}$ Mount Sinai Hospital, ${ }^{3}$ Public Health

Ontario Laboratories, Toronto, ON

BACKGROUND AND OBJECTIVE: Granulomatosis with polyangiitis (GPA; formerly Wegener's disease) is a rare chronic inflammatory disorder that is characterized by granuloma formation in the lung and necrotizingsmall vessel vasculitis. The available evidence indicates that GPA develops as a result of a dysregulated host immune response directed against host neutrophil-derived antigens; however, the precise etiology of this and related diseases remain largely uncertain. Importantly, in addition to genetic influences, it is thought that environmental factors also contribute to susceptibility to GPA. Recent studies have identified a number of human pathogens including Staphylococcus aureus and Burkholderia spp, among others, that have been associated with related vasculitides. Whether these or other pathogens may contribute to GPA pathogenesis is not known and was investigated here.

METHODS: Whole blood was collected and plasma isolated from 24 GPA patients and 7 healthy controls. Plasma endotoxin levels were measured using a commercial ELISA. DNA was extracted from plasma and PCR was used to detect the presence of bacteria using Burkholderia-specific and $16 \mathrm{~S}$ rDNA primers.

RESULTS: No difference was observed in the plasma endotoxin concentration of GPA patients compared to healthy controls. In addition, analysis of the correlation between the inverse ANCA titres and plasma endotoxin level showed no significant difference among GPA patients. Burkholderia DNA (specifically B. pseudomallei, B. thailandensis, and B. cepacia complex) were not detected, nor were any $16 \mathrm{~S}$ rDNA sequences detected in any of the GPA or control samples.

CONCLUSION: In these preliminary studies, we did not detect the presence of bacteria in plasma samples from GPA patients. Additional studies are required to further evaluate the role of bacteria, and possibly other pathogens, in the pathogenesis of GPA.

\section{P17}

EVALUATION OF ANTIGEN DETECTION FOR CONFIRMATORY TESTING OF HEPATITIS C VIRUS INFECTION

PN Levett, D Caswell, J Putz, N Mina, JC Eichhorst, GB Horsman

Saskatchewan Disease Control Laboratory, Regina, SK

OBJECTIVES: Diagnosis of hepatitis C virus (HCV) infection by serological testing requires multiple tests. Many patients with HCV antibodies do not have active infection. We investigated the use of an antigen detection assay for use in the confirmation of $\mathrm{HCV}$ infection.

METHODS: During a five month period, 23,096 serum specimens were tested for HCV antibodies using an automated chemiluminescent immunoassay (Siemens). 1024 specimens that were reactive in this assay were further tested for $\mathrm{HCV}$ antibodies using a recombinant line immunoassay (INNO-LIA) and for HCV antigen using an automated chemiluminescent immunoassay (Abbott).

RESULTS: 600 specimens (58.5\%) were positive for HCV antigen, of which 590 were also positive by INNO-LIA. 8 specimens were indeterminate and 2 were negative by INNO-LIA. Seven of these samples were from patients who subsequently seroconverted, two were not re-tested and one was determined to be a false-positive HCV antigen result. 424 specimens were HCV antigen-negative, of which 384 were positive by INNO-LIA or were from previously confirmed patients. Nine specimens were INNO-LIA indeterminate, of which one patient subsequently seroconverted, and 31 specimens were negative by INNO-LIA.

CONCLUSIONS: Of 1024 serum specimens reactive in the screening assay, 96\% (984) were confirmed positive by either HCV antigen test or immunoblot. Almost $60 \%$ of positive specimens were correctly identified by $\mathrm{HCV}$ antigen testing, allowing faster confirmation of HCV infection at lower cost than a conventional two step antibody detection algorithm. In addition, positive results for hepatitis $\mathrm{C}$ antigen are consistent with active infection and can be used to identify patients for further investigation.

\section{P18}

RECURRENT INVASIVE HAEMOPHILUS INFLUENZAE TYPE A INFECTION IN AN INFANT

K Whyte $^{1}$, PN Levett ${ }^{2}$, GB Horsman ${ }^{2}$, K Chokani ${ }^{3}$, K Hayden ${ }^{1}$, M Shuel $^{1}$, RSW Tsang ${ }^{1}$

${ }^{1}$ Vaccine Preventable Bacterial Diseases, National Microbiology Laboratory, Winnipeg, MB; ${ }^{2}$ Saskatchewan Disease Control Laboratory, Regina; ${ }^{3}$ Prince Albert Parkland Health Region, Prince Albert, SK

BACKGROUND: Before introduction of conjugated Haemophilus influenzae serotype $\mathrm{b}(\mathrm{Hib})$ vaccines into the routine childhood immunisation programs, Hib was a major cause of meningitis in infants and children under the age of 5 . In the post-Hib vaccine era, the epidemiology of invasive $H$. influenzae has changed substantially with most invasive diseases now caused by non-Hib strains, including $H$. influenzae serotype a (Hia) and serotype $f$, as well as non-encapsulated or non-typeable strains. This presentation describes the microbiological characterization of a $H$. influenzae serotype a (Hia) strain involved in causing recurrent invasive infections in an infant. The current knowledge of Hia, including methods of protection will be discussed.

METHODS: Identification and serotyping of $H$. influenzae were done by standard biochemical tests, slide agglutination with serotyping antisera as well as PCR methods. Multi-locus sequence typing (MLST) and pulsedfield gel electrophoresis (PFGE) were used to analyse the molecular genetics of Hia.

RESULTS: Three H. influenzae isolates were recovered from blood and CSF cultures obtained 3 months apart from the infant who succumbed to the second episode of the infection. All 3 isolates belonged to serotype a, sequence type-23, shared identical PFGE profiles, and did not possess the partial deletion involving the capsular IS1016-bexA genes.

CONCLUSION: Hia has emerged as a significant invasive pathogen in the post Hib vaccine era. MLST and PFGE serve as useful techniques for typing of Hia. Many attributes of Hia and the disease it causes bear resemblance to Hib and Hib disease, including the ability to cause recurrent infections. This raises the potential for protection by vaccination and chemoprophylaxis.

\section{P19}

\section{OPTIMIZING THE DIAGNOSTIC YIELD OF JOINT FLUID/ TISSUE CULTURE FOR THE HAMILTON REGIONAL LABORATORY MEDICINE PROGRAM}

\section{Rehan, C Main}

McMaster University and Hamilton Regional Laboratory Medicine Program, Hamilton, ON

OBJECTIVE: The aim of the study was to look at our current joint and tissue culture practices for the HRLMP to determine if these were optimal when compared to expected culture positivity rates published in the literature. Additional questions we hoped to answer were whether or not sonication and/or extending culture incubation duration could potentially increase culture yield without increasing the false positivity rate.

METHODS: In the first phase, we did a literature review to determine published culture positivity rates for joint tissues and fluids and also explored the evidence supporting prolonged culture incubation times and sonication of prosthetic joints. In the next phase, we retrospectively analyzed our hospital database for joint fluid culture and in the final phase, tissue culture, both over a one year period. A total of 406 joint fluids and 56 tissue samples were assessed from culture during this time period. 
RESULTS: Joint fluid culture was positive in 68 samples (16.7\% of total) and tissue culture was positive in 15 samples ( $26.7 \%$ of total). This is lower than would be expected from the literature. Additionally Proprionibacterium acnes, a slowing growing bacteria which is a relatively common cause of joint infection especially in shoulder, was not isolated in any of the cases. According to literature, prolonged incubation of joint fluid/tissue of up to 2 weeks increases the culture yield, especially for slow growing organisms, without increasing the false positivity rate.

CONCLUSION: Based on our low culture positivity rate and the available evidence we would recommend extending the incubation period from 5 days to 7-14 days to increase the culture yield for the slow growing bacteria. We are also exploring the option of sonication of prosthetic joints to increase the yield of culture and help our clinical colleagues in the diagnosis and management of joint infections.

\section{P20}

PUSHING THE LIMITS OF CHEMISTRY POINT-OF-CARE TESTING FOR THE MANAGEMENT OF PATIENTS UNDER INVESTIGATION FOR EBOLA VIRUS DISEASE

LeBlanc $^{1}{ }^{12}$, C Heinstein ${ }^{1}$, J MacDonald ${ }^{1}$, R Gallant ${ }^{1}$, C Roberts ${ }^{1}$, C Jackson ${ }^{1}$, A Lou ${ }^{1,2}$, B Nassar ${ }^{1,2}$, T Hatchette ${ }^{1,2}$

${ }^{1}$ Department of Pathology and Laboratory Medicine, Capital District Health Authority (CDHA); ${ }^{2}$ Department of Pathology, Dalhousie University, Halifax, NS

BACKGROUND: With the recent outbreak in West Africa, hospitals worldwide have been developing protocols for suspect of cases of Ebola virus disease (EVD). Patients with EVD present with a severe gastroenteritis leading to dehydration and electrolyte abnormalities and as such, routine chemistry analysis is essential for patient management. While point of care $(\mathrm{POC})$ testing can be used for rapid chemistry analyses in a containment level 2 laboratories with enhanced precautions, significant delays could ensue before specimens arrive to the laboratory due to additional precautions required during collection and transport. This study evaluated the stability of eight chemistry analytes up to four hours post-collection. METHODS: Blood was collected by venipuncture from 20 healthy volunteers and tested at times 0, 30, 60,90,120, and 240 hours. Approximately $100 \mu \mathrm{l}$ of blood was dispensed into a CHEM 8+ Cartridge and processed on a model 300 i-STAT 1 Analyzer (Abbott Point of Care Inc.).

RESULTS: While the manufacturer recommends testing within $30 \mathrm{~min}$ utes of collection, no significant variation in sodium, potassium, chloride, and creatinine was observed with time points extending up to four hours. For urea, ionized calcium, and bicarbonate, significant differences were observed but the concentration differences were no more than $5 \%$ from the initial time point and would not considered clinically significant. In contrast, glucose concentrations decreased significantly $(\mathrm{P}<0.0001)$ over time at an average rate of $0.0032 \mathrm{mmol} / \mathrm{l}$ per minute $\left(\mathrm{R}^{2}=0.9989\right)$. After 90 , 120 , and $240 \mathrm{~min}$, the decreases would be considered clinically significant at $5.7 \%, 7.8 \%$, and $15.1 \%$, respectively.

CONCLUSIONS: This study provides supporting data suggesting that delays up to four hours can be tolerated, giving ample time for collection and transport of specimens to the clinical laboratory. For glucose, POC testing could still be used, taking into account the collection time and the average rate of decrease.

\section{P21}

\section{INCREASE IN METHICILLIN-SUSCEPTIBLE} STAPHYLOCOCCUS AUREUS WITH REDUCED SUSCEPTIBILITY TO TRICLOSAN OVER THE LAST 20 YEARS M Kissoon ${ }^{1}$, T Ramdass ${ }^{1}$, M Seyed Salehi ${ }^{1}$, A Khan ${ }^{2}$, B Frame ${ }^{2}$, BM Willey ${ }^{1}$, DE Low ${ }^{1,2}$, SM Poutanen ${ }^{1,2}$

${ }^{1}$ University Health Network/Mount Sinai Hospital; ${ }^{2}$ University of Toronto, Toronto, ON

OBJECTIVE: Triclosan as an antibacterial agent in consumer products, such as soaps, deodorants, toothpastes, plastics, and fabrics, has been used widely over the last 20 years. Triclosan targets the enoyl-acyl carrier protein reductase (FabI) which is involved in fatty acid biosynthesis. Triclosan is effective in inhibiting growth of Gram-positive and Gram-negative organisms including S. aureus and Escherichia coli. Widespread use of triclosan has the potential to lead to alterations in FabI resulting in increased triclosan MICs. We previously showed a significant increase in the proportion of clinical methicillin-susceptible S. aureus (MSSA) and methicillin-resistant S. aureus (MRSA) with elevated triclosan MICs (MICs $\geq 1 \mathrm{mg} / \mathrm{L}$ ) isolated from 2004-2005 compared to 1993-1997. This study was completed to determine if triclosan MICs have continued to rise.

METHODS: 308 MSSA and 342 MRSA from 2012-2014 MSSA and MRSA isolates were randomly selected from a Toronto-area clinical laboratory's archived sterile and non-sterile site isolates. Triclosan MICs were determined by agar dilution (AD) following Clinical and Laboratory Standards Institute guidelines. Results were compared to AD MIC results of the sterile and non-sterile 397 MSSA and 315 MRSA from 1993-1997 which had been randomly selected from archived isolates collected from Ontario laboratories as part of surveillance programs; and to AD MIC results of the sterile and non-sterile 436 MSSA and 362 MRSA from 20042005 which were randomly selected from clinical isolates.

RESULTS: The proportion of MSSA with triclosan MICs $\geq 1 \mathrm{mg} / \mathrm{L}$ rose from $0.2 \%$ to $4.6 \%$ to $9.1 \%$ in $1993-1997,2005-2006$, and $2012-2014$, respectively $\left(\mathrm{P}<0.0001, \chi^{2}\right.$ test for trend). There was no significant trend in the proportion of MRSA with triclosan MICs $\geq 1 \mathrm{mg} / \mathrm{L}[1.9 \%, 5.2 \%$, and $0.29 \%$ in 1993 1997, 2005-2006, and 2012-2014, respectively ( $\mathrm{P}=0.1590, \chi^{2}$ test for trend)]. Results were comparable for sterile and non-sterile isolates.

CONCLUSIONS: The proportion of MSSA with triclosan MICs $\geq 1 \mathrm{mg} / \mathrm{L}$ rose significantly from $0.2 \%$ to $9.1 \%$ over the last 20 years from sterile and non-sterile sites. The lack of a similar trend in MRSA, the associated mechanisms of triclosan resistance, and the possible impact on MICs to novel drugs targeting fabI should be further investigated.

\section{P22}

\section{CHARACTERIZING THE RESISTANCE RELATED MUTATIONS IN THE NS3 PROTEASE OF HEPATITIS C-INFECTED ONTARIO PATIENTS}

T Mazzulli ${ }^{1,2,3}$, S Perusini ${ }^{1}$, J Maregmen ${ }^{1}$, O Woods ${ }^{1}$, A Li $^{1}$

${ }^{1}$ Public Health Laboratories, Public Health Ontario; ${ }^{2}$ Department of Laboratory Medicine and Pathobiology, University of Toronto; ${ }^{3}$ Mount Sinai Hospital/University Health Network, Toronto, ON OBJECTIVES: The introduction of antiviral protease inhibitors (PIs) has proven to be an effective treatment for Hepatitis C (HCV) infected patients. However, clinical trials indicate a high rate of baseline mutations in the NS3/4A protease which are known to confer resistance to these agents. No current incidence rates for Ontario patients exist, so we investigated polymorphism rates within a random set of samples submitted for HCV viral load testing and or genotyping.

METHODS: Patient diagnosed with chronic HCV had routine serum samples submitted to our laboratory ( $\mathrm{N}=99$ ). Of these, only the subset with genotype 1a $(\mathrm{N}=76)$ were used in our analysis. A one-step Reverse-transcription (RT) polymerase chain reaction (PCR) followed by a nested amplification of viral HCV RNA was performed. Direct sequencing of the RT-nested PCR product was completed, sequences were assembled, aligned and polymorphisms previously reported to confer resistance to PIs were identified.

RESULTS: Substitutions previously associated with reduced susceptibility to simeprevir were present in the majority of HCV1a cases (51/76 [67.1\%]). The most common was Q80K (46/76 [60.5\%]). Of 51 patients with baseline (pre-treatment) samples, Q80K was present in 29 (56.9\%). Multiple substitutions associated with PI resistance was observe only once as a combination of Q80K and S122G. The Q80K mutation appeared to be more common amongst female patients (17/22 [77.3\%]) compared to male patients (28/51 [54.9\%]). The small number of HIV co-infected HCV patients $(\mathrm{N}=7)$ made it unable to compare polymorphism rates between mono and co-infected patients. Clinically relevant substitutions implicated in resistance to PIs other than simeprevir were uncommon.

CONCLUSIONS: This study is the first to report the rates of mutations in $\mathrm{HCV}$ that confer resistance to PI agents in Ontario. The high baseline rates suggest that pre-treatment testing for the presence of these mutations is essential prior to initiating PI treatment in HCV infected patients. A larger, more comprehensive study is needed to determine other potential parameters that may be associated with PI mutations. 


\section{P23}

IRON RESTRICTION TO CLINICAL ISOLATES OF CANDIDA ALBICANS BY DIBI INHIBITS GROWTH AND INCREASES SENSITIVITY TO AZOLE ANTIFUNGALS

K Savage $^{1}$, M Parquet ${ }^{1}$, D Allan ${ }^{1}$, R Davidson ${ }^{2,3}$, B Holbein ${ }^{1,3}$

${ }^{1}$ Chelation Partners Inc; ${ }^{2}$ Capital District Health Association, QEII

Health Sciences Centre; ${ }^{3}$ Department of Microbiology and

Immunology, Dalhousie University, Halifax, NS

OBJECTIVES: Candida albicans is an opportunistic pathogen associated with human infection frequently treated with azoles. There is some evidence to suggest that interference with iron homeostasis of C. albicans can improve the response to azole antifungals. The objectives of this study were to determine whether the novel iron chelator, DIBI, could inhibit growth of C. albicans in vitro, and whether iron restriction by DIBI could enhance the in vitro activity of fluconazole (FLC) or itraconazole (ITC).

METHODS: Minimum inhibitory concentrations (MICs) for the iron chelators, deferiprone and DIBI, and for FLC and ITC were determined using a broth microdilution method in RPMI. A checkerboard test system was used to compare different combinations of DIBI and FLC or ITC to determine if a synergistic relationship could be established. Each well from the checkerboard system was sub-cultured onto YPD agar at 24 and 48 hour intervals to evaluate recovery from DIBI or azole exposure (alone and in combination). Growth curves were utilized to assess DIBI enhancement of azole activity.

RESULTS: All 3 C. albicans were sensitive to both azoles (FLC 0.12$0.5 \mu \mathrm{g} / \mathrm{mL}$, ITC $0.25-0.5 \mu \mathrm{g} / \mathrm{mL}$ ). MICs to DIBI and deferiprone were $1-4 \mu \mathrm{g} / \mathrm{mL}$ and $64-250 \mu \mathrm{g} / \mathrm{mL}$ respectively. We failed to detect a synergistic relationship using checkboard alone. Recovery of C. albicans was inhibited following $24 \mathrm{~h}$ exposure to $0.5 \mu \mathrm{g} / \mathrm{mL}$ FLC or $32 \mu \mathrm{g} / \mathrm{mL}$ DIBI; or $48 \mathrm{~h}$ exposure to $8 \mu \mathrm{g} / \mathrm{mL}$ FLC or $64 \mu \mathrm{g} / \mathrm{mL}$ DIBI, however, C. albicans was inhibited at lower concentrations of $0.25 \mu \mathrm{g} / \mathrm{mL}$ FLC and $4 \mu \mathrm{g} / \mathrm{mL}$ DIBI in combination. Growth curves demonstrated that C. albicans continued to be suppressed up to 72-96 $\mathrm{h}$ when the azole was combined with DIBI.

CONCLUSIONS: Iron chelation by DIBI can inhibit the growth of C. albicans and significantly enhances the activity of FLC and ITC in vitro.

\section{P24}

\section{WITHDRAWN}

\section{P25}

SNAP—SEPSIS NOW A PRIORITY: DEVELOPMENT AND IMPLEMENTATION OF A SEPSIS ALGORITHM IN THE EMERGENCY DEPARTMENT OF AN ACADEMIC HOSPITAL $\underline{\text { S West }}{ }^{1}$, M Morgan $^{1}$, D Dushenski ${ }^{1}, \mathrm{C}$ McDonald ${ }^{1,2}$,

K Van Den Broek ${ }^{1}$, A Morris ${ }^{1,2}$, S Lapinsky ${ }^{1}$, A Morris ${ }^{3}$

${ }^{1}$ Mount Sinai Hospital; ${ }^{2}$ University Health Network; ${ }^{3}$ Department of Medicine, University of Toronto, Toronto, ON

OBJECTIVES: We developed a sepsis algorithm based on the results of a chart audit of 364 patients presenting to the ER at Mount Sinai Hospital (MSH) in Toronto, Canada, with sepsis in 2010-11. Our algorithm was aimed at improving sepsis management and outcomes by identifying patients early, and providing them with rapid, protocolized care in the MSH emergency department (ED).

METHODS: The prior chart audit was the basis for the development of the Sepsis Now A Priority (SNAP) Recognition and Management algorithm, identifying gaps or deficiencies in care to be addressed. The practice changes implemented were: 1) employment of the SNAP algorithm into the ED with aggressive timelines for clinicians; 2) pre-printed order sets for initial and ongoing management of sepsis; 3) linking patient-tracking board data with collection reports; 4) revised electronic order set for sepsis symptoms; and 5) revised nursing medical directives that align with the algorithm.

RESULTS:_The SNAP algorithm was implemented on July 21, 2014. As of January 1, 2015, there were 378 patients entered into the algorithm at triage. An initial chart audit of 30 patients entered into the algorithm demonstrated that $53 \%(16 / 30)$ of patients had a diagnosis of sepsis. Of those, $19 \%(3 / 16)$ had severe sepsis and 6\% (1/16) had septic shock. Data collected to date has demonstrated that the timelines of the algorithm are being met and that septic patients are being flagged and treated swiftly. Ongoing post-implementation review will evaluate patient outcomes including mortality, morbidity, length of stay and process measures (i.e. time to diagnosis, time to appropriate fluid resuscitation, antibiotics, etc.).

CONCLUSION: The ability to recognize sepsis early is essential to improving outcomes. The intent of the SNAP algorithm is to allow for early recognition of septic patients, with rapid, protocolized care. By implementing this concise quality improvement tool to optimize diagnosis and treatment, we hope to improve outcomes for patients with sepsis. Our algorithm prioritizes identifying patients with possible sepsis over having a specific tool, which would identify fewer patients who do not end up having sepsis.

\section{P26}

\section{WHAT A FLUKE: A CASE OF BILIARY FASCIOLIASIS IN A RECENT IMMIGRANT}

V Meier-Stephenson ${ }^{1}$, T Ishikawa ${ }^{2}$, S Vaughan ${ }^{1}$, S Bass ${ }^{2}$, S Heitman², D Gregson ${ }^{1,3}$

${ }^{1}$ Division of Infectious Disease, Department of Medicine, University of Calgary/Alberta Health Services; ${ }^{2}$ Division of Gastroenterology, Department of Medicine, University of Calgary/ Alberta Health Services; ${ }^{3}$ Medical Microbiology, Calgary Laboratory Services, Calgary, AB

INTRODUCTION: Human fascioliasis is a rare diagnosis in Canada and one made typically only in immigrant populations.

CASE DESCRIPTION: A 20-year-old, previously healthy Ethiopian male immigrated to Canada via Sudan in February 2014. He was seen in the refugee clinic shortly after entry and was found to have scrofula, and subsequently started on antituberculous medications (June 2014). He was also found to have positive schistosomiasis serology for which he was given a prescription for praziquantel (August 2014). In October, he presented to hospital with fevers, vomiting and right upper quadrant pain. Bloodwork showed a normal $\mathrm{CBC}$ without eosinophilia and a cholestatic pattern of liver enzyme elevation. Imaging suggested obstruction, but no stones or masses were visible. Gastroenterology performed an endoscopic retrograde cholangiopancreatography and sphincterectomy which revealed a live, 1.5-cm, flat, leaf-shaped organism!

CONCLUSION: Pathologically the organism was identified as Fasciola hepatica. This case is further unique in that a video of the live fluke has been captured and will be presented. As well, key points of this case, including features for pathologic identification will be discussed.

\section{P27}

AMR, PUBLIC HEALTH, AND KNOWLEDGE TRANSLATION: A FORWARD APPROACH

Lettner $^{1,2,3}$, M Haworth-Brockman 1,2,4, S Balakumar ${ }^{1,2}$

${ }^{1}$ National Collaborating Centre for Infectious Diseases; ${ }^{2}$ International Centre for Infectious Diseases; ${ }^{3}$ University of Manitoba College of Medicine; ${ }^{4}$ University of Winnipeg, Winnipeg, MB

BACKGROUND AND OBJECTIVES: Antimicrobial resistance (AMR) is an increasingly recognized priority for prevention and control of infectious diseases. The causes and solutions of this problem are complex. It is recognized that the control of AMR requires a variety of actions and players from the local to the global level. Moreover, collaboration and coordination of these actions requires leadership from a broad perspective. For these reasons, Public Health is looked to as a key, if not leading, player in an overall strategy. The objectives of this session are 1) to familiarize the audience with the key elements of the Antimicrobial Resistance and Use in Canada: A Federal Framework for Action, and 2) to engage the audience in a discussion of public health approaches to address the problems of AMR, including the importance of knowledge translation and knowledge brokering.

METHODS: The presenter will use the three pillars of the federal framework to describe why this is a problem for the public and for every individual and why the solution is a shared responsibility, requiring leadership and collaboration at all levels of government and across many government and industry sectors. The critical role of knowledge translation and knowledge brokering among Public Health, clinical practice, agriculture and research science will be emphasized. 
RESULTS AND CONCLUSION: As a result of the presentation and discussion, the relationships, roles, and knowledge needs of the varied disciplines and stakeholders will be better understood. This should be a useful step forward for better public health knowledge translation to address the three pillars of the federal framework.

\section{P28 \\ OVERSIGHT OF HUMAN AND ANIMAL PATHOGENS AND TOXINS \\ ML Graham \\ Public Health Agency of Canada, Ottawa, ON \\ BACKGROUND: In 2009, the Human Pathogens and Toxins Act (HPTA) received Royal Assent with the intent to establish a national safety and security regime to protect the health and safety of the public against the risks posed by human pathogens and toxins. With the passage of the HPTA, select provisions came into force to provide interim measures until a complete program and regulatory framework could be developed. \\ METHODS: Regulated and interested parties were consulted over 4 years to develop the proposed Human Pathogens and Toxins Regulations (HPTR) and the Canadian Biosafety Standard (CBS) to support the full implementation of the HPTA scheduled for December 2015. \\ RESULTS: The proposed HPTR would set out a risk-based licensing scheme for facilities conducting controlled activities with human pathogens and toxins. Furthermore a biological safety officer who meets certain knowl- edge requirements set out in the proposed regulations would be designated before a licence can be issued by the Agency. The updated CBS sets out the physical containment, operational practice, and performance and verifica- tion testing requirements for the safe handling and storing of human and terrestrial animal pathogens and toxins. The Agency will also use other pol- icy tools to strengthen pathogen accountability and oversight in Canada. \\ CONCLUSION: The Agency is developing a risk-based program and regulatory framework based on requirements set out in the HPTA, input from stakeholders, policy decisions and other considerations such as domestic and international best practices. This framework facilitates the best and most innovative science at Canadian university and science and technology facilities, enables Canadian public health labs to respond to disease outbreaks and threats of unknown pathogens as efficiently as pos- sible and finally enables Canadian companies to maintain a competitive edge in the economy, all while ensuring activities are conducted in a man- ner that is safe and secure as possible.}

\section{P29 \\ WITHDRAWN}

\section{P30}

THE RETROSPECTIVE STUDY OF THE PREVALENCE AND CLINICAL SIGNIFICANCE OF HEPATITIS B VIRUS PRECORE AND BASAL CORE PROMOTER VARIANTS

$\underline{\text { K Kadkhoda }}^{1}{ }^{12}$, M O'Brien ${ }^{3}$, A Casselman ${ }^{1}$, G Smart ${ }^{1}$, A Gretchen ${ }^{1}$, K Kaita ${ }^{4}$

${ }^{1}$ Cadham Provincial Laboratory; ${ }^{2}$ Department of Medical

Microbiology and Infectious Diseases, University of Manitoba;

${ }^{3}$ PGME, University of Manitoba; ${ }^{4}$ Viral Hepatitis Investigative Unit at the Health Sciences Centre, University of Manitoba, Winnipeg, MB

OBJECTIVES: Hepatitis B Virus (HBV) Precore (PC) and Basal Core Promoter (BCP) variants are recognized for so long but their current prevalence in North America is not known especially among Anti-HBe positive patients chronically infected with $\mathrm{HBV}$ seroconverted. In this study the prevalence of $\mathrm{PC} / \mathrm{BCP}$ mutations and their clinical significance were investigated.

METHODS: A total of 128 patients positive for both HBsAg and Anti-HBe were randomly selected and $\mathrm{PC} / \mathrm{BCP}$ mutations were determined using line probe assay and the patients' chart review was performed to find a potential link with their race/ethnicity, HBV genotype, HBV viral load, liver enzymes, ultrasound, and biopsy results up to ten years prior to the study.

RESULTS: The prevalence of PC and BCP variants in the above cohort of patients was found to be $47.6 \%$ and $62.5 \%$, respectively. Higher age was found to be significantly associated with both APRI $\geq 0.7(P=0.011)$ and with abnormal ultrasound/biopsy results $(P=0.0008)$. Although $\mathrm{BCP}$ variant was significantly associated with APRI $\geq 0.7(P=0.029)$ and not with abnormal ultrasound/biopsy results, a combination of age $\geq 50$ and BCP variant was significantly associated with abnormal ultrasound/biopsy results suggestive of either cirrhosis or HCC. Neither patients' gender nor genotypes showed significant influence on any of these outcomes.

CONCLUSIONS: This study suggests that the prevalence of PC and BCP mutations are more than what is expected which could have been influenced by immigration in the past decade. Considering the potential public health and clinical implications of these variants, long-term multicentre and prospective studies would further unravel the historic enigmas around these variants.

\section{P31}

MYCOPLASMA PNEUMONIAE INFECTION IN CHILDREN LESS THAN FIVE YEARS OF AGE; A 17 YEARS REVIEW

$\underline{\text { Adwan }}^{1,2,3}$, A Bitnun ${ }^{1,2,3}$, AY Shen ${ }^{1,4}$, W Al-Mansouri ${ }^{5}$, S Richardson ${ }^{1,3,4}$, Y Yau ${ }^{1,3,4}$

${ }^{1}$ The Hospital for Sick Children; ${ }^{2}$ Department of Paediatrics, The Hospital for Sick Children; ${ }^{3}$ University of Toronto; ${ }^{4}$ Department of Paediatric Laboratory Medicine, The Hospital for Sick Children, Toronto, ON; ${ }^{5}$ Department of Paediatrics, King Abdul-Aziz Medical City, Jeddah, Kingdom of Saudi Arabia

BACKGROUND: There are limited data on the clinical spectrum of Mycoplasma pneumoniae (MP) infection in children $<5$ years of age. This study aims to describe salient pulmonary and extra-pulmonary manifestations of MP infection in children $<5$ years old.

METHODS: Children $<5$ years old with MP detected by PCR in one or more clinical specimens between Nov. 1996 and Jan. 2014 were identified via the Microbiology Laboratory database. Chart reviews were done on cases identified. Children with an alternative explanation for their clinical presentation were excluded.

RESULTS: 96 children, aged $<5$ years, were identified; 19 were excluded on the basis of an alternative diagnosis. Median age was 36 months ( 2 weeks to 59 months); 7 were $\leq 1$ year of age. Male to female ratio was 1.3:1. MP was detected in 76 respiratory samples (throat swabs [ $n=52]$, nasopharyngeal swabs $[n=12]$, bronchoalveolar lavage $[n=9]$, auger suctions [n=2], pleural fluid [n=1]) and 4 CSF samples. Sixty-seven $(87 \%)$ had symptoms restricted to the respiratory tract, 56 with pneumonia and 4 with asthma exacerbation. Multilobar infiltrates were seen in 13 of $70(19 \%)$ patients who had chest radiographs. There was no difference in the proportion of those with radiographic evidence of multilobar involvement between patients $<24$ months of age and those who were older. Of the $10(13 \%)$ who had extrapulmonary manifestations, 5 had neurologic disease ( 2 encephalitis, 2 ataxia; 1 motor axonal neuropathy), 4 had dermatologic manifestations ( 3 erythema multiforme, 1 serum sickness rash), and 1 had autoimmune hemolytic anemia. Four (40\%) of those with extrapulmonary manifestations did not have documented concurrent or preceding respiratory symptoms. CONCLUSIONS: MP infection can cause significant disease in children $<5$ years of age. Pneumonia and asthma exacerbation are most common, but extrapulmonary manifestations also occur.

\section{P32}

EMERGENCY DEPARTMENT UTILIZATION OF RESPIRATORY VIRUS TESTING FOR FEBRILE YOUNG INFANTS UNDER SIX WEEKS OF AGE

B Burstein 1, AS Dubrovsky ${ }^{1}$, A Greene ${ }^{1}$, Q Quach $^{2,3}$

${ }^{1}$ Department of Pediatric Emergency Medicine; ${ }^{2}$ Department of Pediatric Infectious Diseases, Montreal Children's Hospital; ${ }^{3}$ McGill University, Department of Epidemiology, Biostatistics and Occupational Health, Montreal, QC

BACKGROUND: Well appearing febrile infants with viral illnesses cannot be distinguished from those with occult life-threatening infections by history and physical exam alone. However, infants with confirmed respiratory viruses are at lower risk of serious bacterial infection. This study sought to assess the utilization of respiratory virus testing (RVT) among pediatric ED 
physicians in the evaluation of febrile infants under 6 weeks of age. METHODS: A scenario-based survey describing 2 hypothetical cases of febrile infants without a focus aged 3 and 5 weeks was sent to ED physicians at 15 pediatric tertiary centers across Canada. Participants were asked multiple choice questions to evaluate the use of RVT. In parallel, we performed a single center retrospective analysis of RVT among all infants under 6 weeks evaluated in the ED for sepsis from 2009 to 2013.

RESULTS: Survey response rate was $81 \%(n=171)$. Based on the hypothetical scenarios, RVT was performed more frequently in 3-week infants ( $40 \%$ vs. $29 \%, \mathrm{P}<0.05$ ). Conversely, more ED physicians felt that RVT would have an impact on the management of 5 -week infants (35\% vs. $15 \%, \mathrm{P}<0.001)$. RVT was performed more among physicians reporting test results available under 6 hours compared to those reporting test results available only after 24 hours, for both 3 -week ( $62 \%$ vs. $32 \%, \mathrm{P}<0.01)$ and 5 -week-old infants $(41 \%$ vs. $14 \%, \mathrm{P}<0.01)$. There were significant variations in rates of RVT between centers, ranging from $0 \%-75 \%$ for 3 -weekold infants, and from 0\%-63\% for 5-week-old infants. In clinical practice, among 1292 infants evaluated in a single ED for sepsis, RVT was performed more frequently for infants 4-6 weeks of age than for infants under 4 weeks ( $73 \%$ vs. $58 \%, \mathrm{P}<0.001$ ).

CONCLUSIONS: Utilization of RVT for the evaluation of febrile young infants differs by infant age and among centers. More widespread access to rapid RVT may increase the utilization of this tool in the pediatric ED.

\section{STUDENT POSTER PRESENTATIONS}

\section{SP01 \\ COPD SYMPTOM AND DISEASE SCREENING IN AN HIV POPULATION}

B Ghadaki ${ }^{1}$, N Kronfli ${ }^{1}$, S Haider ${ }^{1,2}$

${ }^{1}$ McMaster University; ${ }^{2}$ Division of Infectious Diseases, Hamilton Health Sciences, Hamilton, $\mathrm{ON}$

BACKGROUND: HIV-positive individuals represent a population that appears to be at a higher risk of developing chronic obstructive pulmonary disease (COPD). In this study, we sought to determine the effects of smoking on respiratory symptoms and smoking related diseases among HIVpositive patients and to determine if symptomatic patients are being appropriately screened for COPD.

METHODS: HIV-positive individuals were asked to complete a selfadministered questionnaire regarding respiratory symptoms and diseases. The effects of smoking on respiratory symptoms and diseases were reported as estimates of odds ratio. The screening criteria used to determine at risk patients for COPD were adapted from the Canadian Thoracic Society (CTS) guidelines.

RESULTS: A total of 247 patients were recruited. The median age was 49 years; $75 \%$ were male and $92 \%$ were currently on combination antiretroviral therapy (cART). Current and former smokers represented $66 \%$ of the population. Smoking had a statistically significant effect on respiratory symptoms including wheeze (OR 4.8 [95\% CI 1.6-14.2]), phlegm production (OR 4.9 [95\% CI 2.2-10.5]), current cough (OR 7.0 [95\% CI 3.0-16.2]), chronic cough $\geq 3$ months (OR 5.2 [95\% CI 2.3-11.8]) and dyspnea (OR 7.2 [95\% CI 1.7-31.2]). Smoking also had a statistically significant effect on respiratory diseases including COPD (OR 4.9 [95\% CI 1.1-21.9]), bronchitis (OR 3.8 [95\% CI 1.9-7.7]), asthma (OR 6.0 [95\% CI 2.0-17.7]) and pneumonia (OR 2.1 ([95\% CI 1.2-4.0]). Among HIV-positive smokers, 40\% met the CTS criteria for COPD screening, while only $12 \%$ of smokers selfreported a diagnosis of COPD and 9\% reported use of inhaled puffers.

CONCLUSIONS: The burden of smoking in the HIV population is significant. HIV-positive smokers are more likely to report both respiratory symptoms and diseases than HIV-positive non-smokers. A discrepancy exists between patients who meet the CTS COPD screening guidelines and those who have been diagnosed with COPD, raising the concern for underrecognition and under-diagnosis of COPD in the HIV-positive population. Our study is limited by self-reporting of symptoms and disease states.

\section{SP02}

WITHDRAWN

\section{SP03}

CHARACTERIZATION OF CANADIAN HOSPITAL INFECTION CONTROL PRACTICES FOR CAREGIVERS AND VISITORS

$\mathrm{G} \mathrm{Chan}^{1}$, G Vomiero' ${ }^{2}$, JV Vayalumkal' ${ }^{2}$

${ }^{1}$ University College Dublin, Dublin, Ireland; ${ }^{2}$ Department of

Pediatrics, Alberta Children's Hospital, University of Calgary,

Calgary, AB

BACKGROUND: Caregivers (parents/guardians) and visitors may be potential vectors in the transmission of Healthcare-Associated Infections (HAIs). This represents a large, uncontrolled population that can access hospital facilities shared between patients and staff. However, compared with Healthcare Workers (HCWs), policies and protocols for this population are less studied and lack standardization.

OBJECTIVES: It is not clear how much caregivers and visitors contribute to transmitting pathogens in hospitals. The objective of this study was to explore the varying practices and policies in Infection Prevention and Control (IPC) directed towards them.

METHODS: An online survey was administered to all 35 members of the Canadian Hospital Epidemiology Committee (CHEC). The 12-item survey consisted of 3 questions related to demographics and 9 related to IPC policies. The survey was conducted and analyzed with SelectSurvey.net (ClassApps, Overland Park, KS). Responses were anonymous.

RESULTS: Of 35 potential responses, 16 (46\%) were completed. All respondents represented acute care facilities, of which $56 \%$ were hospitals with $\geq 500$ beds. All reported use of screening tools on entry, and $62 \%$ screened with ward staff. Favoured IPC measures for caregivers/ visitors included hand hygiene $(100 \%)$, use of PPE $(88 \%)$, and screening-based visit restriction (38\%). The majority $(88 \%)$ had policies for visitor PPE use, and while $92 \%$ encouraged it, $77 \%$ reported that education was provided on proper donning and doffing. All facilities educated caregivers/visitors on IPC measures by using visual materials, and $13(81 \%)$ trained ward staff to teach them. Only 3/16 reported encouraging IPC measures differently between caregivers and visitors; strategies included family meetings, exclusion of non-essential visitors with communicable diseases, and expecting caregivers to practice routine or additional precautions.

CONCLUSION: It is apparent that education to caregivers and visitors is an important approach used by most hospitals represented by CHEC members in Canada. However, it is not clear how compliant caregivers and visitors are with these policies. Furthermore, the degree to which they contribute to the transmission of HAIs is not well understood.

\section{SP04 \\ ANTIMICROBIAL PRESCRIBING PRACTICES IN THE TREATMENT OF SUSPECTED UTI IN THE ELDERLY IN THE EMERGENCY DEPARTMENT: A RETROSPECTIVE SINGLE CENTRE STUDY}

$\underline{\text { C Lata }}^{1}{ }^{1}, \mathrm{R}$ Somayaji ${ }^{1,2}$, DL Holton ${ }^{2}$

${ }^{1}$ University of Calgary Department of Internal Medicine;

${ }^{2}$ Department of Infectious Diseases, Calgary, AB

OBJECTIVES: The elderly population presents a challenge in the diagnosis and treatment urinary tract infection (UTI) and in appropriate antimicrobial treatment. Despite consensus guidelines, this age group are over-diagnosed and over-treated for suspected UTI. Evidence shows no mortality benefit, and significant risk of harm, in treating asymptomatic bacteriuria with antibiotics. Thus, stakes for effective diagnosis and appropriate treatment are high. The clinical features that predispose empiric treatment were observed.

METHODS: All patients aged over 65 years presenting to a local emergency department with a suspected UTI between June 2011 and December 2011 were identified from an administrative database. 458 patient visits were included for analysis. The risk ratio of receiving empiric antibiotics or intravenous therapy specifically was calculated based on various historical, physical exam, and investigational features. 
RESULTS: Of the cohort, $91 \%$ of patients received empiric antibiotics, whereas only $53 \%$ had urine cultures that were positive for an organism (an over-estimate of 'true' UTI). Significant risk factors for receiving empiric treatment were a history of fevers or chills and a positive urine culture (RR 12.0 and 1.9, respectively). Significant risks for receiving IV antibiotics were incontinence, confusion or delirium, fever, positive nitrite on urinalysis, and history of diabetes or neurological disorders (RR 1.6 to 3.8). However, classic symptoms such as dysuria, frequency, and urgency were not significant predictors for receiving empiric antibiotic therapy.

CONCLUSIONS: There was a high prevalence of antibiotic use in this elderly cohort presenting with suspected UTI. Multiple features were associated with increased risk of receiving antibiotics, but were not classic features of UTI. Further studies are required in order to assess the efficacy of these clinical features in accurate diagnosis of UTI in this age group in order to minimize inappropriate antibiotic use.

\section{SP05}

THE INFECTIOUSNESS AND/OR VIRULENCE OF ISONIAZID-RESISTANT VS. SUSCEPTIBLE PULMONARY TUBERCULOSIS: A CASE-CONTROL STUDY IN ALBERTA HY Zhou ${ }^{1,3}$, D Long ${ }^{1}, Z_{\text {Gao }}{ }^{1}$, S Christianson ${ }^{2}$, M Sharma ${ }^{2}$, J Wolfe ${ }^{2}$, C Heffernan ${ }^{1}$, R Long ${ }^{1}$

${ }^{1}$ University of Alberta, Edmonton, AB; ${ }^{2}$ National Reference Center for Mycobacteriology, National Microbiology Laboratory, Public Health Agency of Canada, Winnipeg, MB; ${ }^{3}$ The Provincial Laboratory for Public Health, Edmonton, AB

OBJECTIVE: In Canada, isoniazid (INH) is the most common first-line anti-tuberculosis (TB) drug to which isolates of Mycobacterium tuberculosis are resistant. The aim of this retrospective case control study is to determine whether isoniazid(INH)-resistant strains are as transmissible/ virulent as INH-susceptible stains.

METHODS: Over the 20 years, 1991-2010, all adults (age $>14$ years) with culture-positive pulmonary TB in the Province of Alberta, Canada were identified. From within this cohort, those with phenotypically INH-resistant isolates were identified and defined as "cases". Phenotypic resistance was confirmed and INH- conferring mutations sought in each isolate. From among these that were INH-susceptible patients, two age ( \pm 5 years), sex, population group, smear status and cavitation status "controls" were randomly selected. Conventional and molecular epidemiological tools were then used to identify secondary cases from among contacts. The proportion of contacts that were secondary cases were compared in cases and controls.

RESULTS: A total of 96 pulmonary TB cases were infected with INHresistant TB strains; 76 with high-level and 20 with low-level phenotypic resistance. kat G-only mutations were present in 61 strains (all high-level), inhA-only mutations in 16 strains ( 14 low-level, 2 high-level), both kat $\mathrm{G}$ and inh A mutations in 4 strains ( 3 high-level, 1 low-level) and no mutation or an aphC-only mutation in 15 strains (10 high-level, 5 low-level). Contacts of cases were significantly less likely than contacts of controls to be secondary cases ( 1 of 2203 vs. 20 of 4331), $\mathrm{P}<0.05$.

CONCLUSION: kat $\mathrm{G}$ mutations were associated with high-level INH resistance. INH-resistant strains were less transmissible/virulent than INHsusceptible stains.

\section{SP06}

\section{MEROPENEM MIC PROFILE OF CARBAPENEMASE- PRODUCING ENTEROBACTERIACEAE IN A LOW PREVALENCE REGION}

$\underline{\text { R Fattouh }}{ }^{1}$, N Tijet ${ }^{2}$, AJ McGeer ${ }^{1,3}$, SM Poutanen ${ }^{1,3}$, RG Melano ${ }^{1,2}$, SN Patel ${ }^{1}, 2$

${ }^{1}$ University of Toronto, ${ }^{2}$ Public Health Ontario Laboratories;

${ }^{3}$ Mount Sinai Hospital, Toronto, ON

BACKGROUND AND OBJECTIVE: Over the past decade, transmission of carbapenemases has become a major problem and carbapenemase producing Enterobacteriaceae (CPE) have been identified in countries worldwide. Of significance, infection with CPE has been associated with substantial morbidity and mortality. Thus, there is a critical need to curtail their dissemination. In an effort to better inform CPE screening practices we analyzed the meropenem MIC distribution of a large set of clinical Enterobacteriaceae isolates.

METHODS: A total of 1022 clinical/screening isolates submitted to the Public Health Ontario Laboratories (PHOL), from January 2011 to March 2014, were analyzed. Only isolates displaying a meropenem or ertapenem MIC of $\geq 0.25$ or $1 \mu \mathrm{g} / \mathrm{mL}$ (determined by agar dilution), respectively, were included. Carbapenemase-positive isolates were identified by multiplex PCR. RESULTS: We identified 189 carbapenemase-positive isolates (90 bla $\mathrm{NDM}_{\mathrm{N}}$,

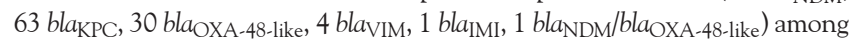
the data set, largely within the Klebsiella spp, Escherichia coli and Enterobacter spp. Interestingly, 15 or $20 \%$ of CPE displayed meropenem MICs within the susceptible range based on current CLSI $(\leq 1 \mu \mathrm{g} / \mathrm{mL})$ or EUCAST $(\leq 2 \mu \mathrm{g} / \mathrm{mL})$ breakpoint tables, respectively. The majority of meropenem-susceptible CPE were observed among E. coli isolates; however, this phenomenon was not exclusive to any one genus. Among the most identified carbapenemase types, a substantial proportion of the OXA-48-positive (40\%/50\% based on CLSI/EUCAST breakpoints) and KPC-positive (22\%/33\%) isolates were found to be meropenem susceptible, whereas few, if any, of the NDMpositive isolates $(0 \% / 2 \%)$ were meropenem susceptible. Notably, application of CLSI screening recommendations captured only $85 \%$ of carbapenemaseproducing isolates, whereas application of EUCAST recommendations captured $>98 \%$ of CPE.

CONCLUSION: Our findings demonstrate that in a region with low carbapenemase prevalence, meropenem-based screening approaches will require a cut-off $\mathrm{MIC}$ near the epidemiological wild-type cut-off in order to achieve near optimal CPE identification.

\section{SP07 \\ CREATION OF A NOVEL PEDIATRIC ANTIMICROBIAL STEWARDSHIP ADVANCED FELLOWSHIP TRAINING PROGRAM}

N Rajapakse $^{1,2}$, JV Vayalumkal ${ }^{1,2,3}$, L Bresee ${ }^{4,5}$, B Dalton ${ }^{4,5}$, T Jadavji ${ }^{1,3}$, C Tsang ${ }^{3,4}$, O Vanderkooi ${ }^{1,3}$, J Conly ${ }^{1,2,5}$

${ }^{1}$ University of Calgary; ${ }^{2}$ Infection Prevention and Control, Alberta Health Services; ${ }^{3}$ Alberta Children's Hospital; ${ }^{4}$ Department of Pharmacy Services, Alberta Health Services; ${ }^{5}$ Foothills Medical Centre, Calgary, AB OBJECTIVE: To describe the development and structure of a Pediatric Antimicrobial Stewardship (AS) Advanced Fellowship program in Canada. BACKGROUND: Though some Canadian training programs with a focus on AS in adult medicine exist, there are currently no programs with a focus on pediatrics.

METHODS: A Pediatric AS Advanced Fellowship program was developed for the 2014-2015 academic year. Recognition by the Post-graduate Medical Education (PGME) office was obtained following submission of a formal application for an advanced fellowship program detailing the structure, objectives and evaluation strategy for the fellowship along with specific CanMeds objectives.

RESULTS: The intent of the fellowship is to prepare a physician with training in Pediatric Infectious Diseases for a career trajectory in pediatric AS by learning the core knowledge required to perform AS related program development, research and clinical work. The program is comprised of multidisciplinary collaboration with the adult/pediatric pharmacy teams and Infection Prevention and Control teams at tertiary care sites in the city. The trainee is also involved in the local and provincial AS committees and other projects including review of local clinical guidelines, education and collaborative projects. Foundational theory in the principles of AS is gained through a longitudinal research component, working with experienced physicians/pharmacists, and independent learning through accredited antimicrobial stewardship courses. Successful completion of the fellowship will be acknowledged with a certificate awarded by the PGME office. Application for Royal College recognition as an "Area of Focused Competence" is in progress.

CONCLUSIONS: This novel training program provides a unique opportunity to develop skills in pediatric antimicrobial stewardship and could serve as a prototype for other pediatric centres to develop similar programs. 


\section{SP08}

EMERGING ANTIMICROBIAL RESISTANCE AMONG STAPHYLOCOCCUS PSEUDINTERMEDIUS, A POTENTIAL ZOONOSES OF CANINE ORIGIN

\section{R Priyantha, J Rubin}

University of Saskatchewan, Saskatoon, SK

BACKGROUND: Staphylococcus pseudintermedius is a ubiquitous colonizer and opportunistic pathogen of dogs. S. pseudintermedius is coagulase positive, biochemically similar to and indistinguishable from S. aureus on CHROMagar S aureus and Denim Blue Agars. Although rarely recognized (and likely under-identified), human S. pseudintermedius infections are typically zoonotic, stemming from contact with dogs. The emergence of antimicrobial resistance, including methicillin resistance (MRSP), is therefore of interest to physicians and veterinarians.

METHODS: Pharyngeal and rectal swabs were taken from 100 healthy dogs presenting for vaccination to the Western College of Veterinary Medicine in Saskatoon, SK. Samples were cultured for staphylococci and S. pseudintermedius was identified biochemically including tests for acetoin and hyaluronidase production which differentiate it from S. aureus. Antimicrobial MICs were determined and interpreted using the CLSI guidelines. Methicillin resistance was confirmed by PCR amplification of the mecA gene.

RESULTS: $78 \%$ of dogs were colonized with S. pseudintermedius including $7 \%$ with MRSP. No isolates resistant to fluoroquinolones, rifampin, nitrofurantoin, vancomycin, linezolid, daptomycin or quinupristin + dalfopristin were identified nor was inducible clindamcyin resistance. The most common resistance phenotypes were penicillin + ampicillin (31.7\% of isolates) and pan-susceptibility (30.3\% of isolates). Resistance to tetracycline $(20.8 \%$ of isolates), erythromycin, clindamycin, trimethoprim + sulfamethoxazole, gentamicin and chlroamphenicol (all $\leq 5 \%$ of isolates) was also found. None of the MRSP colonized dogs had received antimicrobials in the previous 6 months, indicating a community reservoir.

CONCLUSIONS: Compared to a similar $S$. pseudintermedius surveillance study conducted in 2008, resistance (including MRSP) was more frequently identified. The introduction of high throughput highly discriminatory bacterial identification technologies such as MALDI-TOF, may increasingly result in the identification of $S$. pseudintermedius from human infections. Physicians should be aware of this potential zoonoses and consider its infection control implications.

\section{SP09}

POSITIVE IMPACT OF INFECTIOUS DISEASE CONSULTATION ON QUALITY OF CARE, MORTALITY AND LENGTH OF STAY IN STAPHYLOCOCCUS AUREUS BACTEREMIA: RESULTS FROM A LARGE MULTICENTER COHORT STUDY

A Bai ${ }^{1}$, A Showler ${ }^{2}$, L Burry ${ }^{3,4}$, M Steinberg ${ }^{3}$, D Ricciuto ${ }^{2,5}$, T Fernandes $^{6}$, A Chiu ${ }^{6}, \mathrm{~S}$ Raybardhan ${ }^{7}$, M Science ${ }^{8}$, E Fernando ${ }^{2}$, G Tomlinson 2,9, C Bell2,3,10, A Morris ${ }^{2,3,9}$

${ }^{1}$ North York General Hospital; ${ }^{2}$ University of Toronto; ${ }^{3}$ Mount Sinai Hospital; ${ }^{4}$ Leslie Dan Faculty of Pharmacy, Toronto; ${ }^{5}$ Lakeridge Health, Oshawa; ${ }^{6}$ Trillium Health Partners, Mississauga; ${ }^{7}$ North York General Hospital; ${ }^{8}$ Hospital for Sick Children; ${ }^{9}$ University Health Network; ${ }^{10}$ Institute for Clinical Evaluative Sciences, Toronto, ON OBJECTIVES: We assessed the impact of Infectious Disease (ID) consultation on management and outcome in Staphylococcus aureus bacteremia (SAB). METHODS: A retrospective cohort study examined consecutive SAB patients from 6 academic and community hospitals between 2007 and 2010. Quality measures of management including echocardiogram, repeat blood culture, removal of infectious foci, and antibiotic therapy were compared between ID consultation (IDC) and no ID consultation (NIDC) groups. A competing risk model with propensity score adjustment was used to compare in-hospital mortality and time to discharge.

RESULTS: Of $847 \mathrm{SAB}$ patients, 506 (60\%) patients received an ID consultation and $341(40 \%)$ patients did not. During hospital stay, 371 (73\%) IDC patients and 191 (56\%) NIDC patients received an echocardiogram $(\mathrm{P}<0.0001)$. Repeat blood cultures in $2-4$ days of bacteremia were performed in 207 (41\%) IDC patients and 107 (31\%) NIDC patients $(\mathrm{P}=0.0058)$. There was no statistical difference in removal of infectious foci between the two groups. For patients who were alive when the antibiotic course was completed, 285/422 (68\%) IDC patients and 141/262 (54\%) NIDC patients received the appropriate duration of antibiotic therapy $(\mathrm{P}=0.0004)$.

During hospital stay, 204 (24\%) patients died: 104 (21\%) IDC patients and 100 (29\%) NIDC patients. Matched by propensity score, ID consultation had a sub-distribution hazard ratio (sHR) of 0.72 (95\% CI: 0.52-0.99, $\mathrm{P}=0.0451)$ for in-hospital mortality and 1.28 (95\% CI: 1.06-1.56, $\mathrm{P}=0.0109$ ) for being discharged alive.

CONCLUSIONS: ID consultation is associated with adherence to quality measures, reduced in-hospital mortality and earlier discharge in SAB patients.

\section{SP10}

PROSPECTIVE OBSERVATIONAL STUDY OF DIAGNOSIS OF URINARY TRACT INFECTION AND RESPONSE TO THERAPY IN LONG TERM CARE

C Penney ${ }^{1}$, S Wakeham ${ }^{1}$, G Compton ${ }^{2}$, A McKim² ${ }^{2}$, O O'Keefe ${ }^{2}$, B Barrett $^{2}$, L Nicolle ${ }^{2}$, P Daley ${ }^{1}$

${ }^{1}$ Memorial University; ${ }^{2}$ Eastern Health Region, St John's, NL

BACKGROUND: Prevalence of asymptomatic bacteriuria among residents of long-term care (LTC) facilities is $40 \%$, and is a source of inappropriate antibiotic use.

OBJECTIVES: To determine the signs and symptoms associated with positive urine culture and if antibiotic therapy is associated with functional improvement.

METHODS: LTC residents were prospectively observed following urine culture submission.

RESULTS: 174 consecutive specimens from 6 LTC facilities were considered and 101 specimens from 101 residents were eligible. Mean age was 84.0 years (SD 8.6), 79.2\% were female, with a mean of 1.8 comorbidities per patient (SD 1.0). Baseline ADL score was 11.9 (SD 8.7), with zero representing total independence and 28 representing total dependence. $38 / 101$ specimens $(37.6 \%)$ had significant growth. Reasons for collection ranged from one to seven reasons with dysuria, change in character of urine and change in mental status being the most common. Most episodes did not have vital signs or blood testing performed in order to apply published diagnostic criteria. Using univariate regression, two predictors were associated with culture positivity (male sex, RR 5.58, 95\% CI = 1.23-25.43, change in mental status, RR $13.83,95 \% \mathrm{CI}=1.81-105.81)$. Antibiotic therapy was prescribed in $48 / 101(47.5 \%)$ of episodes, but treatment decisions did not correlate with significant growth (kappa=0.44). 19/48 (40\%) of treatments were given to residents without significant growth and $9 / 53$ (17\%) of episodes with significant growth were not treated. 4/28 (14.3\%) of treatments given were reported as resistant. Baseline, 2 day and 5-7 day ADL scores did not significantly change in treated or untreated groups. CONCLUSIONS: The observed rate of significant growth is approximately the same as the expected rate of asymptomatic bacteruria. Antibiotic treatment did not lead to functional improvement. Restricting access to urine culture may be a future strategy to improve antibiotic stewardship.

\section{SP11 \\ MOLECULAR EPIDEMIOLOGY OF VANCOMYCIN HETERORESISTANT COAGULASE-NEGATIVE STAPHYLOCOCCI IN A NEONATAL INTENSIVE CARE UNIT IN QUEBEC}

\Chong $^{1}$, S Lévesque ${ }^{2}$, A Blanchard ${ }^{3}$, C Laferriere $^{4}$, C Quach ${ }^{1,3}$ ${ }^{1}$ McGill University; ${ }^{2}$ Laboratoire de santé publique du Québec/ Institut national de santé publique du Québec; ${ }^{3}$ Montreal Children's Hospital; ${ }^{4} \mathrm{CHU}$ St Justine Hospital, Montreal, QC OBJECTIVE: Coagulase negative staphylococci (CoNS) have emerged as a leading cause of bloodstream infections (BSIs) in ICUs, in particular in premature neonates. The study aimed to describe the molecular and epidemiological characteristics of heterogeneous vancomycin intermediate coagulase negative staphylococci (hVICoNS) central line associated BSI (CLABSI) in a single tertiary-care NICU in Québec. 
METHODS: Between November 2009 and April 2014, all CoNS causing CLABSI were identified in a single tertiary care NICU in Québec through the laboratory information system and the infection control database. Heterogeneous resistance to vancomycin (hV) was determined by both the Macro E-test and E-test GRD. Antibiotic susceptibility to daptomycin and linezolid was determined by E-test, following the manufacturer's recommendations. Clonal relationships were determined by pulsed-field gel electrophoresis (PFGE). Population analysis profile/area under the curve (PAP/AUC), as the gold standard for $\mathrm{hV}$, is being performed.

RESULTS: Eighty-eight CoNS strains were collected. Decreased vancomycin susceptibility was identified in all isolated strains. The most common species of hVICoNS causing CLABSI were Staphylococcus epidermidis (96.6\%), S. warneri (2.3\%), and S. capititis (1.1\%). Eighty-two hVICoNS strains (93.2\%) were susceptible to daptomycin, whereas only $52(59.1 \%)$ of isolated strains were susceptible to linezolid.

PFGE revealed 27 distinct patterns (A to $A B$ ), with 6 strains untypeable. More than half of the strains ( 45 of $88 ; 51.1 \%$ ) belonged to 3 patterns: $E(n=8 ; 9.1 \%), M(n=26 ; 29.5 \%)$, and $O(n=11 ; 12.5 \%)$. PFGE pattern E strains spanned from 2009-2014, pattern M strains spanned from 2010 . 2014, and pattern O strains spanned from 2011-2013.

CONCLUSIONS: hVICoNS seems more common than currently realized in the NICU, although PAP/AUC results are still pending. Treatment of hVICoNS CLABSI with linezolid may no longer be appropriate, with $40.9 \%$ of strains demonstrating resistance. PFGE pattern E, M, and O strains spanned several years, indicating that certain clones can become endemic to the NICU.

\section{SP12}

\section{INCREASING INCIDENCE OF GROUP C AND GROUP G STREPTOCOCCAL BACTEREMIA ATTRIBUTED TO INCREASED BACTERIAL VIRULENCE FACTORS AND POOR OUTCOMES}

\section{$\underline{\text { L Lother }}^{1}$, Y Keynan ${ }^{1,2}$, P Degagne ${ }^{3}$, P Lagacé-Wiens ${ }^{2,3}$, MR Mulvey ${ }^{2,4}$, I Martin ${ }^{4}$ \\ ${ }^{1}$ University of Manitoba, Department of Internal Medicine; \\ ${ }^{2}$ University of Manitoba, Department of Medical Microbiology and Infectious Disease; ${ }^{3}$ Diagnostic Services Manitoba; ${ }^{4}$ National Microbiology Laboratory, Winnipeg, MB}

BACKGROUND: $\beta$-hemolytic streptococci are responsible for several human diseases, including skin and soft tissue infection, bacteremia, and streptococcal toxic shock syndrome. Mortality rates in bacteremia have been reported up to $20 \%$. We have described an increased incidence of group C and G streptococcal (GCS and GGS, respectively) bacteremia from January 2007 to December 2012 in our center. We hypothesize acquired virulence factors accounts for increased pathogenicity and poor clinical outcomes.

OBJECTIVES: To identify streptococcal virulence factors in our GCS and GGS blood isolates compared to controls.

METHODS: The study population included all patients with blood cultures for GCS and GGS in two tertiary care centers from January 2012 to December 2014. Polymerase chain reaction (PCR) was performed on a subset of 15 patients and 15 non-invasive controls obtained from throat culture. 5 superantigens previously described in invasive group A streptococcal bacteremia (speA, speG, speK, smeZ, ssa) were explored. Whole genome sequencing, protein profiling via MALDI-Tof, clinical parameters such as demographics, co-morbidities, disease severity and outcomes via retrospective chart review will be completed in the near future.

RESULTS: 90 blood and 33 throat isolates were retrieved. Of the blood isolates, 57 (63\%) and 33 (37\%) were Lancefield group G and C, respectively. Of the throat isolates, $17(52 \%)$ and $16(48 \%)$ were Lancefield group G and C, respectively. MALDI-Tof mass spectrometry confirmed all but one blood isolate as Streptococcus dysgalactiae grp species. The other was identified as Streptococcus equi. Superantigen genes speA, speG, speK, smeZ, and ssa were not detected by PCR in our subset.

CONCLUSIONS: No invasive pathogenic factor has been identified in this study to account for the increased incidence. Further studies are underway to analyze whole genome sequencing and protein profiling in our cohort.

\section{SP13}

ANTIMICROBIAL STEWARDSHIP IN PRIMARY CARE: AN EVALUATION OF THE MANAGEMENT OF ACUTE UNCOMPLICATED CYSTITIS IN A FAMILY HEALTH TEAM M Rostom $^{1}$, P Marr ${ }^{1,2}$, D Kwan ${ }^{1,2}$, C Papoushek ${ }^{1,2}$, L Dresser ${ }^{1,2}$, C Lemieux ${ }^{1,2}$

${ }^{1}$ University Health Network; ${ }^{2}$ University of Toronto, Toronto, ON OBJECTIVES: To compare prescribers' self-reported practices with actual practices in the management of acute uncomplicated cystitis and to assess adherence to published guidelines.

METHODS: Phase 1 consisted of a case-based survey to determine prescribers' self-reported management of cystitis and use of guidelines. Phase 2 was a retrospective chart review, analyzing actual management of cystitis in women aged 16 years and older in an academic family health team (FHT). RESULTS: Phase 1: Seventeen (40\%) prescribers at the FHT completed the survey. As part of initial case management in the survey, 7 (41\%) respondents treated empirically, $13(76 \%)$ performed a dipstick urinalysis, and $7(41 \%)$ sent urine for culture and sensitivity (C\&S) testing. Empiric antibiotic choice was consistent with guideline options for all survey respondents. Four (24\%) respondents selected duration of empiric therapy that did not match guideline recommendations. A broad spectrum agent was chosen by $5(29 \%)$ respondents when provided with urine C\&S results. Phase 2: Fifty-five patients were included in the retrospective chart review. Fifty $(91 \%)$ had documented signs and symptoms of cystitis. Dipstick urinalysis was performed for $44(80 \%)$ patients. Urine for C\&S was sent from $45(82 \%)$ patients, of which $33(73 \%)$ samples were positive. Antibiotic prescriptions were written for 54 (98\%) patients; 47 (87\%) empiric and $7(13 \%)$ based on C\&S results. Choice of antibiotic was in accordance to guidelines for $46(85 \%)$ patients. Duration of therapy was not concordant with guidelines for 19 (35\%) of prescriptions.

CONCLUSIONS: In this study, self-reported and actual management of acute uncomplicated cystitis were not concordant. Adherence to guidelines was lower in actual compared to self-reported practice with respect to antimicrobial selection and duration of therapy. There is opportunity for improving management of cystitis with better understanding of why actual practice does not match reported practice.

\section{SP14}

\section{WITHDRAWN}

\section{SP15}

CLOSTRIDIUM DIFFICILE INFECTIONS (CDI) IN CHILDREN: ARE WE OVERESTIMATING RATES JUST BECAUSE OF THE DIAGNOSTIC METHOD USED?

M Alghounaim, Y Longtin, M Gonzales, M Bilhete, C Quach McGill University, Montreal, QC

BACKGROUND: C. difficile is the most common cause of hospital-associated diarrhea. Numerous assays are available to diagnose CDI. Detection of the toxin B gene (tcdB) by PCR is becoming increasingly popular, replacing toxin production detection by enzyme-linked immunosorbent assay (ELISA) or cell cytotoxicity assay. Given the increase in CDI rates following PCR implementation in many settings, our objective was to determine the proportion of pediatric patients diagnosed as CDI by PCR who would have also been diagnosed as CDI by ELISA, and to compare the clinical characteristics of PCR+/ELISA+ vs. PCR+/ELISA- patients.

METHODS: Using the microbiology laboratory information system, stool samples from children seen at the Montreal Children's Hospital (MCH) that were positive for C. difficile by PCR (BD GeneOhm ${ }^{\circledR}$ ) between October 2010 and July 2014 were identified. Using frozen stool specimens (single freeze-thaw cycle), an ELISA (TOX A/B II ${ }^{\circledR}$ ) for toxin A and B was performed. A retrospective chart review was conducted to obtain demographic and clinical data, using a standardized case report form. Duplicate samples were excluded. Chi-square was used to compare proportions and student t-test for continuous variables. The study was approved by the MCH Research Ethics Board.

RESULTS: Overall, 154 of 310 identified stool samples had been kept (49.7\%). Eighteen samples were excluded (17 duplicates and one patient 
was never seen at the $\mathrm{MCH}$ ). A total of $136 \mathrm{PCR}$ positive samples underwent ELISA testing: 54 were positive $(40 \%)$ for toxin A or B. The average age of the entire cohort was 8.5 years (SD 6.2). There was no $(p>0.05)$ significant difference in age, gender, CDI clinical manifestation, previous medical problems, and management between patients positive or negative by ELISA. However, patients with positive ELISA results were more likely to have had a recent exposure to antibiotics $(67.9 \%$ vs. $50 \%, \mathrm{P}=0.04$ for ELISA + and -, respectively).

CONCLUSION: In our pediatric population, $60 \%$ of patients with CDI diagnosed by PCR had no toxin detectable by ELISA. PCR+/ELISApatients are less likely to have received an antibiotic recently than $\mathrm{PCR}+$ / ELISA+ patients. These results highlight the need to standardize laboratory criteria for the diagnosis of CDI in children.

\section{SP16}

SURVEILLANCE OF HUMAN PARAINFLUENZA TYPES 1-4 AND ASSOCIATION WITH ACUTE LOWER RESPIRATORY TRACT INFECTIONS IN HOSPITALIZED CASES, ALBERTA, CANADA $(2010$ - 2013)

S Fathima $^{1,2}$, K Simmonds $^{3}$, J Invik ${ }^{4}$, AN Scott ${ }^{3}$, SJ Drews ${ }^{1,5}$ ${ }^{1}$ Provincial Laboratory for Public Health, Edmonton, AB; ${ }^{2}$ London School of Hygiene and Tropical Medicine, London, UK; ${ }^{3}$ Alberta Ministry of Health, Edmonton; ${ }^{4}$ University of Calgary, Calgary; ${ }^{5}$ University of Alberta, Edmonton, $\mathrm{AB}$

OBJECTIVE: To describe the association of the Human Parainfluenza virus types (PIV 1-4) in hospitalized cases, with acute respiratory illnesses such as bronchiolitis, croup, pneumonia, and mixed illnesses in Alberta, Canada.

METHODS: ProvLab Alberta tests all respiratory samples for influenza A/B. Influenza A/B- negative specimens from non-community patients undergo the Respiratory Virus Panel classic assay, which detects PIV 1-4. Positive PIV specimens were merged with Alberta Public Health administrative databases to define cases and hospitalization status, using unique patient identification numbers for all Alberta patients. ICD-9 codes were used to determine if cases had one event of croup, bronchiolitis, pneumonia, or mixed events (any combination of the three acute respiratory illnesses). All statistical analysis was done using SPSS (Version 19.0.0, IBM Corp(C) 2010).

RESULTS: 1204 hospitalized PIV 1-4 cases were identified. PIV 3 was the most common type in Alberta, 44.8\% (539/1204) followed by PIV 1, 25\% (301/1204). Of all PIV 1-4 cases, pneumonia was the most common acute respiratory illness 33.5\%, (403/1204) followed by bronchiolitis $19.5 \%$, (235/1204). The majority of croup cases, $58.9 \%$ (103/175) were due to PIV 1 (Fischer's Exact $=166.242, \mathrm{P}<0.001$ ) while pneumonia cases $49.1 \%$, (198/403) were most likely to be associated with PIV 3 (Fischer's Exact $=14.937, \mathrm{P}=0.002$ ). There was no statistical significance between the different PIV types and bronchiolitis or mixed events; however PIV 3 was the most common type in both clusters of illness; bronchiolitis, $46.8 \%$ (110/235), mixed events, 44.5\% (49/110).

CONCLUSIONS: This study shows association of PIV 1-4 types with specific illnesses including croup and pneumonia. Periodic surveillance of PIV types can be used to evaluate the epidemiology and burden of PIV in hospitalized cases with acute respiratory illnesses.

\section{SP17 \\ CLOSTRIDIUM DIFFICILE INFECTION IN SOLID ORGAN TRANSPLANT RECIPIENTS AT A TERTIARY CARE CENTRE DURING 2006-2013}

N Zelyas, S Smith, K Doucette

University of Alberta, Edmonton, $\mathrm{AB}$

OBJECTIVES: Clostridium difficile infections (CDIs) represent a serious burden of disease in hospitalized patients. Solid organ transplant (SOT) recipients are severely immunosuppressed and therefore may be more susceptible to infection and development of severe CDI. This study characterized first-time CDIs in SOT recipients over an eight year period.

METHODS: SOT recipients admitted to the University of Alberta Hospital with a CDI during 2006-2013 were identified retrospectively. Charts were reviewed to determine CDI rates for different patient groups.
CDI severity, treatment regimens, previous antibiotics, and 30-day outcomes were also determined.

RESULTS: Ninety-five first-time CDIs occurred in SOT recipients over 2006-2013, during which 1825 SOTs were performed. Liver recipients made up the largest proportion of infected patients (44.2\%) followed by lung and kidney recipients, respectively. Nearly half $(44.2 \%)$ of CDIs occurred within 30 days of transplant. Severe CDI was present in $50.5 \%$ of patients and only $15.8 \%$ of patients received guideline-concordant therapy. Treatment regimens often differed from guidelines in multiple ways, with the most common deviations being extended duration of therapy, using lower metronidazole doses, and using metronidazole alone for severe CDIs. Within 30 days prior to CDI diagnosis, $44.2 \%$ of patients received fluoroquinolones, $44.2 \%$ received $\beta$-lactam/ $\beta$-lactamase inhibitors, and $36.8 \%$ received carbapenems. Within 30 days following first-time CDI diagnosis, two patients were admitted to the ICU due to CDI, two had colectomies, and none died.

CONCLUSIONS: The rate of CDIs in SOT recipients was not higher than in other hospitalized patients and had little variation from year to year. Liver recipients made up the bulk of infected patients, which is not surprising considering the anatomic site of transplantation. Unsurprisingly, SOT patients with CDI had been exposed to numerous antibiotics; as well, they were likely to suffer severe disease when infected. Low guidelineconcordant therapy may be due to increased caution in treating an already compromised group of patients as well as lack of guideline awareness. The minimal number of adverse outcomes may indicate that SOT patients are generally subjected to a high level of care.

\section{SP18}

SAFETY OF THE LIVE-ATTENUATED INTRANASAL INFLUENZA VIRUS (LAIV) VACCINE IN CHILDREN AND ADOLESCENTS WITH CYSTIC FIBROSIS AND THEIR HEALTHY SIBLINGS

$\underline{C}$ Boikos $^{1}$, G Deserres ${ }^{2,3}$, LC Lands ${ }^{4}$, G Scheifele ${ }^{5}$, FD Boucher ${ }^{6}$, M Chilvers $^{7}$, P Daigneault ${ }^{8}, C^{2}$ Quach $^{1,3,9,10}$

${ }^{1}$ Department of Epidemiology, Biostatistics \& Occupational Health, McGill University, Montreal; ${ }^{2}$ Department of Social and Preventive Medicine, Laval University; ${ }^{3}$ Institut national de santé publique du Québec; ${ }^{4}$ Department of Pediatrics, Division of Respiratory Medicine, The Montreal Children's Hospital, Montreal; ${ }^{5}$ Division of Infectious and Immunological Diseases, Department of Pediatrics, University of British Columbia; ${ }^{6}$ Department of Pediatrics, Division of Infectious Diseases, Centre Mère-Enfant Soleil du CHU de Québec, Québec, QC; ${ }^{7}$ Division of Respiratory Medicine, Department of Pediatrics, Faculty of Medicine, University of British Columbia, Vancouver, BC; ${ }^{8}$ Department of Pediatrics, Division of Respiratory Medicine, Centre Mère-Enfant Soleil du CHU de Québec, Québec; ${ }^{9}$ Department of Pediatrics, Division of Infectious Diseases, The Montreal Children's Hospital; ${ }^{10}$ McGill University Health Centre, Vaccine Study Centre, Research Institute of the MUHC, Montreal, QC

OBJECTIVE: To determine the safety of LAIV in children with cystic fibrosis (CF) and their healthy siblings.

METHODS: During the 2013-14 influenza season, we recruited 94 children and adolescents with CF and 19 healthy siblings, aged 2-19 years, from 3 CF clinics across Canada. Prospective active surveillance for solicited and unsolicited adverse events following immunization (AEFIs) was performed for 56 days post-LAIV administration. The proportion of AEFIs reported throughout follow-up and during days 0-6 was explored using contingency tables.

RESULTS: Four participants (3.5\%), all with CF, were hospitalized during the follow-up period; only 1 , occurring on day 16 post-LAIV administration, due to pulmonary exacerbation, was determined to be potentially attributable to LAIV. The following solicited symptoms were reported during days 0-6 of follow-up; no facial edema was reported. 


\begin{tabular}{lcccc}
\hline & \multicolumn{2}{c}{ Participants with CF } & Healthy \\
\cline { 2 - 3 } \cline { 2 - 3 } Symptoms & First LAIV (n=28) & Year 2 of LAIV $(\mathbf{n}=\mathbf{6 6})$ & & $(\mathbf{n}=\mathbf{1 9})$ \\
\hline Tired & $30 \%$ & $12 \%$ & $26 \%$ \\
Headache & $25 \%$ & $17 \%$ & $32 \%$ \\
Worse cough & $21 \%$ & $9 \%$ & $11 \%$ \\
Chills & $18 \%$ & $3 \%$ & $0 \%$ \\
Myalgia & $14 \%$ & $3 \%$ & $5 \%$ \\
Nausea/Vomiting & $11 \%$ & $0 \%$ & $0 \%$ \\
Arthralgia & $7 \%$ & $3 \%$ & $5 \%$ \\
Eye redness & $4 \%$ & $0 \%$ & $5 \%$ \\
Incident wheezing & $4 \%$ & $0 \%$ & $0 \%$ \\
\hline
\end{tabular}

CONCLUSION: LAIV appears to be well tolerated in children and adolescents with CF. We did not detect a signal for oculo-respiratory syndrome post-LAIV in this population, as previously postulated. Study results support a national vaccine recommendation for preferential use of LAIV in this population that will reduce influenza-related morbidity and mortality in all children with chronic diseases.

\section{SP19}

SEVERE SKIN LESIONS INDUCED BY A PREDOMINANT CA-MRSA STRAIN USA300 ARE ASSOCIATED WITH A UNIQUE NEUTROPHIL RESPONSE AND INVOLVEMENT OF PROTEINASE ACTIVATED RECEPTORS (PARS)

LZhang1 ${ }^{1}$ J Conly ${ }^{1,2,3}$, J McClure ${ }^{2}$, K Wu ${ }^{1}$, D Tambalo ${ }^{1}$, B Petri ${ }^{1}$, D Barber ${ }^{3}$, S Elsayed ${ }^{4}$, G Armstrong1 , M Hollenberg1 , K Zhang1,2,3 ${ }^{1}$ University of Calgary; ${ }^{2}$ Alberta Health Services; ${ }^{3}$ Calgary Laboratory Services, Calgary, AB; ${ }^{4}$ University of Western Ontario, London, ON

OBJECTIVES: The mechanisms of severe skin lesions induced by a predominant CA-MRSA strain USA300 remain unknown. We established a murine intradermal infection model capable of demonstrating dermatopathological differences between USA300 and other MRSA strains, and capable of revealing new potential mechanisms contributing to development of severe skin lesions.

METHODS: BALB/c mice were monitored for skin lesions and systemic infection after intradermal injection with virulent MRSA USA300, USA400 and avirulent M92. Biopsies of the core cutaneous lesion were subjected to detailed histopathological examination ( $\mathrm{H} \& \mathrm{E}$, Gram, and Esterase staining). Neutrophil infiltration and adhesion/emigration were quantified by myeloperoxidase (MPO) assay and spinning disk confocal microscopy, respectively. RB6-8C5 antibody was used to deplete neutrophils 1 day prior to (early) or post (late) infection. PAR1/2 KO and C57BL/6 WT mice were used to study the role of PARs in USA300 dermatopathology.

RESULTS: Skin lesions induced by USA300 uniformly presented as extensive ulcers with a profound inflammatory cell infiltrate. In contrast, USA400 and M92 only caused localized cutaneous infection. In this model, no systemic infection was observed, although Gram staining indicated deeper bacterial invasion in USA300-infected tissues. Esterase staining and MPO assay confirmed that USA300 induced greater neutrophil infiltration than other strains $(\mathrm{P}<0.05)$. Live cell imaging displayed significantly increased neutrophil (but not CD4 cell) adherence and emigration in USA300-infected mice compared to other strains (all $\mathrm{P} \leq 0.05$ ). Early (but not late) neutrophil-depletion exacerbated skin lesion sizes $(\mathrm{P} \leq 0.05)$, and both early and late depletion resulted in extensive bacterial dissemination and systemic infection in USA300-infected mice. Results with PARnull mice showed that USA300 induced smaller lesion sizes with less neutrophil infiltration in PAR1-KO than WT mice. Similar lesion size but faster healing with decreased neutrophil infiltration was observed in PAR2-KO relative to WT mice.

CONCLUSIONS: We were able to demonstrate the key clinical characteristics between CA-MRSA USA300 and other MRSA strains in a murine intradermal infection model. In part, the mechanism of
USA300-induced severe skin lesions is related to over-activation of neutrophils possibly through activation of PARs.

\section{SP20 \\ SURVEILLANCE OF ANTIMICROBIAL USE IN QUÉBEC ACUTE CARE HOSPITALS: A DREAM OR ALMOST REALITY? É Fortin $^{1,2}$, DG Thirion ${ }^{3}$, L Bergeron ${ }^{4}$, M Gonzales ${ }^{5}$, J Papenburg5 , M Dionne $^{2}$, A Fortin ${ }^{2}$, C Quach ${ }^{1,2,5}$ \\ ${ }^{1}$ McGill University, Montréal; ${ }^{2}$ Institut national de santé publique du Québec, Québec; ${ }^{3}$ Université de Montréal, Montréal; ${ }^{4}$ Centre Hospitalier Universitaire de Québec, Québec; ${ }^{5}$ The Montreal Children's Hospital, Montréal, QC}

OBJECTIVES: In 2011, the Québec Ministry of Health (MSSS) issued a directive to acute care hospitals to implement surveillance of antimicrobial (AM) use in admitted patients. We thus aimed to describe 1) available pharmacy data; 2) hospitals' actual practices in qualitative and quantitative surveillance of AM use; 3) hospitals' motivation to perform surveillance of AM use.

METHODS: A web-based questionnaire was sent to chief pharmacists in the province of Quebec acute care hospitals for them to complete or forward to the pharmacist in charge of AM use surveillance.

RESULTS: Of 110 questionnaires sent, 44 were at least partly filled (40\%), representing 49 hospitals. A database describing AM use was available in 43 hospitals (6 had individual-level data only, 30 had aggregated data only and 7 had both). Ninety percent of hospitals (36/40) had a qualitative surveillance program or were about to implement one ( 4 had implemented it before 2011); most monitored indication (89\%), AM choice $(89 \%)$, dosage $(78 \%)$, duration of treatment $(78 \%)$ and route $(72 \%)$. Eighty percent of hospitals (32/40) had a quantitative AM use surveillance program (QASP) or were about to implement one (5 had implemented it before 2011); most of these hospitals (84\%) monitored defined daily doses of AM per patient-days, but non distributed and non administered doses were analyzed differently across hospitals. In $80 \%$ of hospitals, the participant was favourable to the implementation of a provincial QASP. Foreseen problems were the lack of human, financial and information technology resources; comparisons between hospitals were viewed as both a methodological challenge and useful information.

CONCLUSION: AM use surveillance has been implemented in most participating hospitals, but databases are not readily available and indicator definitions vary. However, most participants had a positive view regarding an eventual provincial surveillance program.

\section{SP21}

\section{USEFULNESS OF FECAL CALPROTECTIN AND SERUM} PROCALCITONIN IN PREDICTING A POSITIVE C. DIFFICILE TOXIN GENE POLYMERASE CHAIN REACTION ASSAY K Popiel $^{1}$, M Miller ${ }^{2}$

${ }^{1}$ McGill University Health Centre, ${ }^{2}$ Jewish General Hospital, Montreal, QC

BACKGROUND: Nosocomial diarrhea is multi-factorial. With C. difficile infection (CDI) being high on the differential, testing for C. difficile is routine in hospitalized patients with diarrhea. Given the high sensitivity of current PCR assays for CD toxin gene detection, novel "inflammation" assays could be used as adjunctive tests to discriminate between colonization and CDI.

METHODS: Stool samples received for routine CD-PCR underwent additional testing with two fecal calprotectin assays (Calprotectin (CPT) and Calprotectin High-Range (CPT-HR)). Patient sera were tested to measure procalcitonin (PCT) levels. Retrospective chart review was performed to determine associated clinical parameters. Receiver-operating characteristics were drawn to evaluate the performance of these 3 assays on predicting a positive CD-PCR (CD-PCR+). Informed consent was provided by all patients.

RESULTS: In all, 64 subjects (44 CD-PCR+; 20 CD-PCR-) were enrolled. There was no significant difference with regards to gender $(53 \%$ male) and age distribution between groups; 47 (73\%) were $\geq 60$ years old. $\mathrm{CD}-\mathrm{PCR}+$ cases had a higher white cell count $(\mathrm{P}<0.01)$ but did not differ 
significantly from CD-PCR- cases with regards to serum albumin, serum creatinine, temperature, presence of abdominal pain, presence of inflammatory bowel disease or concomitant antibiotic therapy. Mean fecal calprotectin values were higher in CD-PCR+ samples $(\mathrm{P}<0.001)$, as was the mean serum PCT ( $\mathrm{p} 0.021$ ) in those subjects. The area under the curve (AUC) for CPT-HR predicting CD-PCR + was 0.82 (95\% CI 0.70-0.94) with a sensitivity of $87 \%$ and a specificity of $75 \%$ at a HR-CPT of $135 \mathrm{mcg} / \mathrm{g}$. CPT was less specific with an AUC 0.8 (CI 0.67-0.92), sensitivity $87 \%$, specificity $55 \%$, at a CPT of $81.5 \mathrm{mcg} / \mathrm{g}$. The AUC for PCT was not significant.

CONCLUSIONS: Elevated fecal CPT-HR is a valid predictor of CD-PCR+ in a hospitalized patient with diarrhea, however further studies are required to determine its quality as a marker of CDI versus colonization.

\section{SP22}

\section{SUCCESSFUL USE OF INTRATHECAL COLISTIN IN A CASE OF PSEUDOMONAS AERUGINOSA VENTRICULITIS: A CASE REPORT AND BRIEF REVIEW OF THE LITERATURE}

D Whellams ${ }^{1}$, N Irfan ${ }^{2}, Z_{\text {Z Chagla }}{ }^{3}$

\section{${ }^{1}$ Laboratory Medicine Residency Program, McMaster University;} ${ }^{2}$ Hamilton Health Sciences; ${ }^{3}$ St Joseph's Healthcare, Hamilton, ON BACKGROUND: The use of intrathecal antibiotics is rare, but may represent an additional treatment option in cases of ventriculitis with multi-drug resistant (MDR) organisms or in patients with persistently positive cultures. METHODS: We searched PubMed for English language articles using the terms "intrathecal," "colistin," and "Pseudomonas". We present a case where intrathecal colistin was used successfully in a case of Pseudomonas aeruginosa ventriculitis, as well as a summary of six additional similar cases from the literature.

RESULTS: The most common presenting diagnosis was subarachnoid haemorrhage in five of seven patients. In all seven cases, intrathecal therapy was used in conjunction with systemic antibiotic therapy and removal or externalization of the infected catheter. In two cases, the renal side effects of intravenous (IV) antibiotics prompted a switch to intrathecal colistin. Only one study reported side effects with intrathecal colistin in the form of self-limited paresthesias of the arm. In two cases, intrathecal colistin therapy resulted in cerebrospinal fluid (CSF) sterilization when intravenous (IV) antibiotics alone had failed to do so. In our case, the CSF was sterilized by day seven of IV colistin alone, but a subsequent rise in CSF leukocytes suggested persistent infection, prompting intrathecal therapy. In three patients where IV and intrathecal colistin were used concomitantly, CSF sterilization occurred by day 2-4 of therapy, and no recurrence occurred.

CONCLUSIONS: Intrathecal colistin has been successfully employed in CNS shunt infections with multi-drug resistant Pseudomonas aeruginosa in conjunction with IV antibiotics resulting in rapid CSF sterilization, and physicians should be aware of this potential strategy. Patients should be monitored for neurological side effects during therapy.

\section{SP23 \\ PREVALENCE OF ANEMIA IN THE SASKATCHEWAN POPULATION WITH HIV INFECTION}

\section{G Shumilak, K Ng, K Williams, S Stewart}

University of Saskatchewan, Saskatoon, SK

BACKGROUND: Anemia is a common hematologic complication in patients with Human Immunodeficiency Virus (HIV) infection. It is an independent risk factor for disease progression and adverse clinical outcomes that include increased morbidity and mortality. Appropriate management of anemia in patients with HIV infection improves functional outcomes and quality of life. The prevalence of anemia, risk factors for developing anemia, and rates of intervention for patients with anemia are currently unknown in the Saskatchewan population with HIV infection. METHODS: A retrospective chart review of 292 adult Saskatchewan patients with HIV infection was performed. The primary endpoint was the presence of anemia as defined by the World Health Organization. Six variables and their impact on the prevalence of anemia were examined. Rates of anemia investigation and intervention by physicians were also assessed.
RESULTS: The current prevalence of anemia in patients with HIV infection is $26.3 \%$ (95\% CI, [21.2\% - 31.4\%]). Anemia was present in $40 \%$ of patients prior to initiating Highly Active Anti-Retroviral Therapy (HAART) and 14\% following effective therapy with a 26\% net reduction. Multivariate analysis demonstrated that female sex (OR 2.0; 95\% CI, 1.083.74, $\mathrm{P}=0.028$ ), First Nations and Métis ethnicity (OR 2.3; 95\% CI, 1.074.91, $\mathrm{P}=0.032$ ), and lack of HAART (OR 3.2; 95\% CI, 1.75-5.99, $\mathrm{P}=0.0002$ ) were associated with significantly increased risk of developing anemia. HCV co-infection (OR 1.9; 95\% CI, 0.96-3.75, P=0.065), other ethnic minorities (OR 3.35; 95\% CI, 0.85-13.15, $\mathrm{P}=0.083$ ), and rural habitation (OR 1.2; 95\% CI, 0.62-2.17, $\mathrm{P}=0.652$ ) were not associated with increased risk of developing anemia. Of patients with anemia, $18.4 \%$ $(95 \% \mathrm{CI},[9.5 \%-27.3 \%])$ had appropriate investigations and $16 \%(95 \% \mathrm{CI}$, $[7.5 \%-24.5 \%])$ had a documented anemia management plan.

CONCLUSION: Anemia is a negative prognostic marker that is prevalent in the Saskatchewan population with HIV infection. Female sex, First Nations and Métis ethnicity, and lack of HAART are significant risk factors for developing anemia. More aggressive strategies are required to investigate and manage anemia in patients with HIV.

\section{SP24 \\ RAPID IDENTIFICATION OF BACTERIA IN POSITIVE BLOOD CULTURES USING SMUDGE PLATE PREPARATION AND MALDI-TOF MS SYSTEM}

Y Chen $^{1}$, V Porter ${ }^{2}$, S Mubareka ${ }^{1,2}$, L Kotowich ${ }^{2}$, A Simor ${ }^{1,2}$

${ }^{1}$ Department of Laboratory Medicine and Pathobiology, University of Toronto; ${ }^{2}$ Department of Microbiology, Sunnybrook Health Sciences Centre, Toronto, ON

OBJECTIVES: Matrix-assisted laser desorption ionization time of flight mass spectrometry (MALDI-TOF MS) is a novel method for rapid identification of bacteria and fungi in clinical microbiology laboratories. We evaluated the performance of MALDI-TOF MS for the rapid identification of bacteria directly from positive blood cultures using "smudge plate" preparations. METHODS: Blood cultures were incubated in the Bactec 9240 system (Becton Dickinson). When a blood culture flagged as positive, a $3 \mathrm{~mL}$ aspirate from the blood culture bottle was obtained, microorganisms were separated from blood cells and concentrated in a serum separator. The bacterial concentrate was then planted as a lawn on a chocolate agar ("smudge") plate and incubated at $35^{\circ} \mathrm{C}$ in $\mathrm{CO} 2$. After 1,2 and 4 hours incubation, organisms were recovered by sweeping the smudge plate, and inoculated on a target slide for identification by Vitek MS (BioMérieux) MALDI-TOF. The identification from MALDI-TOF MS was compared to that from conventional phenotypic identification methods. Discrepancies were resolved using MALDI-TOF MS on the colony isolated from the routine subculture and ultimately, the $16 \mathrm{~S}$ rRNA sequencing method.

RESULTS: We prospectively examined 400 positive clinical blood cultures with 248 Gram positive isolates and 152 Gram negative isolates. The rate of correct identification was $90 \%$ overall to both species and genus level, $97 \%$ for Gram negative isolates, $85 \%$ for Gram positive isolates. It also correctly identified all 6 anaerobes. Among the correctly identified isolates, $72 \%$ of them were identified with 1 hour incubation, and $87 \%$ with 2 hours incubation, and additional $13 \%$ after 4 hours incubation. The rate of discordant ID was $1.8 \%$ and no ID $8.5 \%$, mainly from Gram positive isolates.

CONCLUSIONS: In conclusion, combination of smudge plate preparation and VITEK MALDI-TOF MS system is a simple and efficient way to provide identification of bacteria directly from positive blood cultures within 1 to 4 hours. 
SP25

ASSESSMENT OF THE MANAGEMENT OF SUSPECTED URINARY TRACT INFECTIONS FOR IDENTIFICATION OF TARGETED ANTIMICROBIAL STEWARDSHIP INTERVENTIONS

${\underline{C} \mathrm{Le}^{1,2}}^{1,}$ T MacLaggan ${ }^{1}$, M Kuhn ${ }^{1}$, T Wilcox ${ }^{1}$, J Murray ${ }^{1}$, D Smyth ${ }^{1,3}$

${ }^{1}$ The Moncton Hospital, Moncton, NB; ${ }^{2}$ Department of Family

Medicine, Dalhousie University; ${ }^{3}$ Department of Medicine,

Dalhousie University, Halifax, NS

BACKGROUND: Inappropriate management of suspected urinary tract infections (UTI), such as treating asymptomatic bacteriuria (AB), leads to emergence of antimicrobial resistance and secondary complications including infection with Clostridium difficile.

OBJECTIVES: This study examined empiric antimicrobial selection for suspected UTI to identify target interventions for stewardship initiatives. METHODS: Retrospective chart review was completed for 344 consecutive inpatient urine cultures. Data were collected regarding clinical indications and rationale for empiric UTI antimicrobial therapy.

RESULTS: 278 patients met eligibility criteria and underwent chart review. $190(68.3 \%)$ patients were female, and average age was 73.7 years. $224(80.6 \%)$ patients were given antimicrobial therapy. The three leading empiric antimicrobials prescribed were ciprofloxacin $(n=71,31.7 \%)$, nitrofurantoin $(n=39,17.4 \%)$, and trimethoprim-sulfamethoxazole $(n=34,15.2 \%)$. Detailed review of the medical record led to a diagnosis of $\mathrm{AB}$ in 170 (61.2\%) patients. Of patients diagnosed with $\mathrm{AB}, 119$ (70.0\%) were treated with antimicrobials. In 37 (13.3\%) of the charts reviewed, the only documented abnormality was a nursing note of abnormal urine color or odor. This led to subsequent $\mathrm{AB}$ treatment in $25(67.6 \%)$ of patients. $78(28.1 \%)$ charts reviewed did not have a documented physician order for urine submission. The presence of an MD order was significantly associated with appropriateness to submit urine for culture $(46.0 \%$ vs $24.4 \%, \mathrm{P}=0.002$ ).

CONCLUSIONS: Our results indicate high levels of fluoroquinolone use and high levels of $\mathrm{AB}$ treatment, driven in large part by inappropriate and non-physician directed specimen submission. These results will be used to design and prospectively evaluate an institutional UTI stewardship bundle directed at reducing fluoroquinolone use and $\mathrm{AB}$ treatment, which will involve physician and nurse education, as well as pharmacyand lab-based interventions.

\section{SP26}

CLINICAL FEATURES AND OUTCOMES OF INFLUENZA INFECTION IN COMMUNITY DWELLING OLDER ADULTS PRESENTING TO SIX URBAN EMERGENCY DEPARTMENTS

$\underline{L}$ Goodliffe $^{1}$, P Lam ${ }^{1,2}$, B Colemand ${ }^{1,2}$, K Green ${ }^{1}$, J Powis ${ }^{3}$, D Richardson $^{4}, \mathrm{~K} \mathrm{Katz}^{5}$, B Borgundvaag ${ }^{1}$, AJ McGeer ${ }^{1}$ ${ }^{1}$ Mount Sinai Hospital; ${ }^{2}$ University of Toronto; ${ }^{3}$ Toronto East General Hospital, Toronto; ${ }^{4}$ William Osler Health System, Brampton; ${ }^{5}$ North York General Hospital, Toronto, ON

OBJECTIVES: To describe the clinical features, outcomes of influenza infection and health care utilization for individuals 60 years and older with laboratoryconfirmed influenza presenting to emergency departments (ED) in Ontario. METHODS: We identified community-dwelling older adults presenting to six EDs with influenza-compatible symptoms during the 2011/12 and 2012/13 influenza seasons. Clinical characteristics were collected from patient interviews and chart review. Influenza vaccination history was collected; nasopharyngeal swabs were tested for influenza. Follow-up was conducted with cases up to 30 days after symptom onset.

RESULTS: Of 1337 participants, 147 (11\%) had flu (94 AH3N2, 12 AH1N1, 4 A (not subtyped), 37 B). The most commonly reported symptoms were cough (93\%), fatigue (92\%), (84\%) and weakness; $44 \%$ of patients had a measured temperature $\geq 37.5^{\circ} \mathrm{C}$ at time of triage. CDC and PHAC influenza-like illness (ILI) definitions captured $32 \%$ and $23 \%$ of all cases, respectively. Among cases, $83(55 \%)$ were hospitalized with a median length of stay of 5 days (IQR: 3-7 days); four patients (3\%) died. Of the 101 patients at home on day 30 from symptom onset, 28 (28\%) were not well enough to return to regular activities. Median time to return to regular activities was 18 days ( $\mathrm{n}=134$; range 2 to $>30$ days).
CONCLUSIONS: Older adults commonly present with influenza with non-specific symptoms (such as cough, weakness \& fatigue) and the standardized definitions fail to capture the majority these individuals. The majority of older adults with influenza who present to the ED are hospitalized, and recovery is prolonged.

\section{SP27}

ENGAGING INFECTIOUS DISEASES AND MICROBIOLOGY RESIDENTS IN IMPROVING THE VALUE OF HEALTHCARE: CHOOSING WISELY

DR MacFadden ${ }^{1}$, WL Gold ${ }^{1}$,, I Al-Busaidi ${ }^{1}$, J Craig ${ }^{1}$, D Petrescu ${ }^{1}$, I Saltzman ${ }^{1}$, JA Leis ${ }^{1,3}$

${ }^{1}$ University of Toronto; ${ }^{2}$ University Health Network, ${ }^{3}$ Sunnybrook Health Sciences Centre, Toronto, ON

BACKGROUND: Rising costs present a major threat to the sustainability of healthcare delivery. Resource stewardship is increasingly becoming an expected competency of physicians. We used the Choosing Wisely framework to introduce resource stewardship at a national educational retreat for Infectious Diseases (ID) and Microbiology (M) residents.

METHODS: During the 2014 Annual Canadian ID and M Resident Retreat in Toronto, we engaged ID $(n=50)$ and M $(n=17)$ residents from 11 Canadian universities to participate in a modified Delphi panel. Participants were asked in advance of the retreat to submit up to five practices that ID and $\mathrm{M}$ specialists should not routinely perform due to lack of proven benefit and/or potential harms to patients. Submissions were discussed in small and large group forums using iterative, electronic polling until consensus was reached on five practices. A follow-up survey at two-months was performed. RESULTS: Consensus was reached on the following practices that were considered to be of low-value and may be considered for elimination: (i) repeat diagnostic imaging in all cases of vertebral osteomyelitis; (ii) serial CD4 measurements in virologically-controlled HIV patients; (iii) tuberculin skin testing in the diagnosis of active tuberculosis; (iv) transesophageal echocardiography in all cases of nosocomial Staphylococcus aureus bacteremia; and (v) use of parenteral antibiotics when highly-bioavailable oral antibiotics options are available in appropriate clinical situations. Twenty participants (32\%) completed the follow-up survey. The majority of respondents $(75 \%)$ felt that the session was at least as relevant as other sessions at the retreat, with $95 \%$ indicating that at least some of the material discussed was new to them. Since returning to their home institutions, $45 \%$ of respondents have incorporated what they learned into their daily practice; 4 (20\%) reported that they have considered initiating a project related to the session; and $1(5 \%)$ reported having initiated a project.

CONCLUSIONS: This educational forum demonstrated that trainees can become actively engaged in the identification and discussion of lowvalue practices and guidelines. Embedding teaching about resource stewardship into residency training programs, as demonstrated at our national retreat, will be necessary to improve the value of care offered by the future members of our profession.

\section{SP28}

INCIDENCE AND OUTCOMES OF HOSPITAL-ACQUIRED C. DIFFICILE INFECTION AT A LARGE COMMUNITY HOSPITAL BEFORE AND AFTER A MULTIPRONGED PREVENTION AND MANAGEMENT INITIATIVE

\section{$\underline{L}$ Ryan $^{1}$, T Oommen ${ }^{2}$, J McCarten ${ }^{2}$, A Adumattah ${ }^{2}$, D Ricciuto ${ }^{1,2,3}$}

${ }^{1}$ University of Toronto, Toronto; ${ }^{2}$ Lakeridge Health, Oshawa;

${ }^{3}$ Queen's University, Kingston, ON

OBJECTIVES: The aim of this study is to determine the impact of a multifaceted intervention, with an emphasis on antimicrobial stewardship, to prevent and manage hospital-acquired C. difficile infection (HA-CDI).

METHODS: We conducted a retrospective before and after cohort study to evaluate the impact of a multimodal intervention to reduce C. difficile infection at a large, community hospital. The intervention included initiating a facility-wide C. difficile outbreak and utilizing an Incident Management Team to rapidly engage administrators and leaders and to disseminate education to staff. Other elements during the outbreak included: enhanced environmental services; "toolkit" use by nursing staff to help assess and manage potential cases; 
and a C. difficile pre-printed order set. Antimicrobial Stewardship (ASP) utilizing audit-feedback was instituted on high-risk medical wards. ASP is the only intervention that remained fully active after the outbreak was declared over. We compared the pre-intervention period of July 2010-September 2012 with the post-intervention period of October 2012-July 2014. HA-CDI was defined according to public reporting definitions in Ontario. Incident data was acquired from an Infection Control database. Detailed chart review was performed to gather demographic, comorbidity, treatment and outcome data. RESULTS: A total of 173 cases of HA-CDI occurred during the study period. A reduction in HA-CDI incident rates was observed after implementation of the intervention $(0.29$ vs. 0.17 per 1000 patient-days; $\mathrm{P}<0.001)$. Overall all-cause $(0.62$ vs. 0.19 per 10,000 admissions; $\mathrm{P}<0.001)$ and attributable $(0.36$ vs. 0.09 per 10,000 admissions; $\mathrm{P}<0.05)$ 30-day mortality in patients with HA-CDI decreased significantly post-intervention. Overall hospital-wide antimicrobial use decreased significantly (183 vs. 148 DOT per 1000 patient-days; $\mathrm{P}<0.001$ ). No significant differences in demographics, comorbidities, or appropriateness of treatment were noted before and after the intervention.

CONCLUSIONS: A multimodal intervention resulted in a significant, sustained decline in incident HA-CDI cases at a large, community hospital. A significant sustained decrease in antimicrobial utilization likely helped to maintain lower HA-CDI rates.

\section{SP29 \\ THE CONTINUED RISE OF EXTENDED-SPECTRUM B-LACTAMASE-PRODUCING ESCHERICHIA COLI IN CANADIAN HOSPITALS: CANWARD 2007-2013}

A Denisuik $^{1}$, HJ Adam ${ }^{1,2}$, P Lagacé-Wiens ${ }^{1,2}$, P Simner ${ }^{2}$, MR Mulvey ${ }^{1,3}$, M Baxter ${ }^{1}$, M Gilmour ${ }^{1,3}$, JA Karlowsky ${ }^{1,2}$, DJ Hoban ${ }^{1,2}$, GG Zhanel ${ }^{1}$

${ }^{1}$ University of Manitoba; ${ }^{2}$ Diagnostic Services Manitoba; ${ }^{3}$ National Microbiology Laboratory, Winnipeg, MB

OBJECTIVE: To assess the prevalence, patterns of antibiotic resistance, and molecular characteristics of ESBL-, AmpC-, and carbapenemase-producing EC and KPN isolated from Canadian hospitals.

METHODS: 6,606 EC and 2,058 KPN were collected from January 2007 to December 2013 as part of the ongoing CANWARD national surveillance study. Antimicrobial susceptibility testing was performed according to CLSI guidelines and putative ESBL-, AmpC-, and carbapenemase-producers were identified. All putative isolates were characterized by PCR and sequencing to detect resistance genes and by PFGE to assess clonal spread. The EC ST131 clone was identified by an allele-specific PCR for the pabB gene.

RESULTS: The prevalence of ESBL-EC [2007: 3.4\%, 2013: 9.5\%], AmpC-EC [2007: 0.7\%, 2013: 3.1\%], and ESBL-KPN [2007: 1.5\%, 2013: $5.7 \%$ increased significantly during the study period, with all three antibiotic resistant organisms reaching peak incidence in 2013. Antimicrobials demonstrating the greatest activity against ESBL-EC, AmpC-EC, and ESBL$\mathrm{KPN}$ in this study were colistin, amikacin, ertapenem, and meropenem, whereas $78.8 \%, 34.9 \%$, and $66.7 \%$ of ESBL-EC, AmpC-EC, and ESBLKPN, respectively, were multidrug resistant. The prevalence of the ST131 clone was higher in ESBL-EC (56.9\%) compared to AmpC-EC (31.7\%; $P<0.001)$. CTX-M-15 was the dominant genotype in both ESBL-EC and ESBL-KPN (66.5\% and 48.0\%, respectively), whereas the dominant genotype in AmpC-EC was CMY-2 (53.2\%). KPC-3 represents the dominant genotype among carbapenemase-producers $(n=4)$. In total, 5 isolates demonstrating a meropenem MIC $\geq 1 \mu \mathrm{g} / \mathrm{mL}$ were collected in 2013, this in comparison to a total of 5 such isolates for the years 2009 to 2012 combined. CONCLUSIONS: The prevalence of ESBL- and AmpC-producing EC and KPN increased significantly between 2007 and 2013. The prevalence of carbapenem-resistant Enterobacteriaceae remains low in Canada.

\section{SP30}

INPATIENT CARE OF COMMUNITY ACQUIRED PNEUMONIA: OPPORTUNITIES FOR IMPROVEMENT

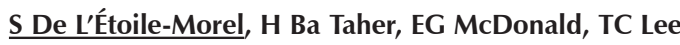

McGill University, Montreal, QC

OBJECTIVE: Community acquired pneumonia (CAP) remains a common reason for hospitalization and guideline concordant care may be associated with improved outcomes. We describe a local population of patients with CAP to identify areas for quality improvement.

METHODS: We conducted a retrospective review of 130 consecutive adult patients with CAP admitted between January 2013 and June 2014 to our clinical teaching units ( 97 beds of 917 total). Patients were identified through our antimicrobial "self-stewardship" program wherein the indication is documented for all antibiotics. Empyema and healthcare-associated pneumonia were excluded.

RESULTS: The median age was 75 (IQR 61-83) with a median length of stay 9.5 days (IQR 6-14). The most common comorbidities were chronic obstructive pulmonary disease $(33 \%)$, diabetes $(29 \%)$ and coronary artery disease (25\%). Hypoxia (36\%), tachycardia (11\%) and hypotension (5\%) were rare on presentation. The chest $\mathrm{x}$-ray was interpreted as lobar consolidation in $68(52 \%)$. The PSI was greater than 3 in 74 (57\%). Blood and sputum cultures were rarely positive $(7 / 83,8 \%$ and $17 / 52,33 \%)$. Streptococcus pneumoniae was the most common organism (20\%). Viral PCR was positive in 20 of $81(62 \%)$. Antibiotics were administered within 8 hours in $82 \%$ and were guideline-concordant in $34 \%$. A common "transgression" was the use of piperacillin-tazobactam in $25 \%$. Therapy exceeded 7 days in $67 \%$. C. difficile infection occurred in $13(10 \%)$, ICU transfer in $6(4.6 \%)$, and inpatient death in only $4(3.1 \%)$. All deaths occurred in those with a PSI of 4 or greater.

CONCLUSION: We describe a cohort of patients admitted with CAP to our institution. Microbiological diagnosis was rare and departures from our institution's guidelines were common. Outcomes were excellent with few deaths or transfers to ICU but C. difficile was a frequent and potentially avoidable morbidity.

\section{SP31 \\ FEASIBILITY OF UV-C LIGHT DISINFECTION IN A CANADIAN HOSPITAL}

$\underline{\text { Y Emond }}^{1}$, I Deans ${ }^{2}$, C O'Neill ${ }^{2}$, D Mertz ${ }^{1,2}$

${ }^{1}$ McMaster University; ${ }^{2}$ Hamilton Health Sciences, Hamilton, ON

BACKGROUND: Devices using UV light have been shown to be effective in deactivating multiple microorganisms and potentially reducing the transmission of pathogens in hospitals. We conducted a study to evaluate a pulsed xenon ultraviolet (PX-UV) technology disinfection system $\left(\right.$ Xenex $\left.^{\circledR}\right)$ to evaluate feasibility of use and its impact on hospital acquired infections (HAI).

METHODS: The study took place on two internal medicine units of 35 and 31 beds respectively, including 4 private rooms each. The plan was to deploy the PX-UV machine (provided by Xenex ${ }^{\circledR}$ ) following routine environmental cleaning for all isolation discharge and all patient bathrooms daily for 6 months on each unit while the other ward served as a control, with a cross-over for another 6 months. Bed spaces in multi-bed rooms were isolated using opaque blanket before each disinfection to allow utilization of PX-UV while the room was not vacated. The efficacy was measured by comparing the rate of HAI.

RESULTS: Feasibility was hampered by the fact that hanging an opaque blanket before disinfecting non-private rooms was too time consuming. Therefore, the machine was used almost exclusively for private rooms after terminal cleaning and all bathrooms daily. Also, due to staff limitation, the machine could only be used between $8 \mathrm{am}$ and $4 \mathrm{pm}$ on business days. No difference in the rates of HAI were detected with or without the intervention (Table 1).

\section{TABLE 1}

Mean $\mathrm{HAl}$ rate

\begin{tabular}{|c|c|c|c|}
\hline & $\begin{array}{l}\text { Intervention } \\
\text { group }\end{array}$ & $\begin{array}{l}\text { Control } \\
\text { group }\end{array}$ & $\mathrm{P}$ value $(95 \% \mathrm{Cl})$ \\
\hline Clostridium difficile infection & 1.03 & 0.71 & $3.67(-0.59,+1.21)$ \\
\hline $\begin{array}{l}\text { Methicillin resistant } \\
\text { Staphylococcus aureus }\end{array}$ & 1.28 & 0.82 & $1.04(-1.04,+1.95)$ \\
\hline $\begin{array}{l}\text { Vancomycin-resistant } \\
\text { enterococcus }\end{array}$ & 3.60 & 2.71 & $0.15(-1.69,+3.48)$ \\
\hline
\end{tabular}

CONCLUSION: Because of the significant percentage of non-private rooms, deployment of the (PX-UV) disinfection system proved to be impractical and did not affect HAI outcomes in our institution. 
SP32

NON-POLIO ENTEROVIRUS TYPES ASSOCIATED WITH ACUTE FLACCID PARALYSIS - A SYSTEMATIC REVIEW

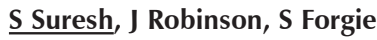

University of Alberta, Edmonton, AB

OBJECTIVES: With polio vaccination programs having almost eliminated wild type poliovirus as a cause of acute flaccid paralysis (AFP), there is increased interest in AFP due to other enteroviruses (EV) such as EVD 68.

This study aims to characterize the nonpolio enteroviruses (NPEV) associated with acute flaccid paralysis (AFP), transverse myelitis (TM) or Guillain Barre Syndrome (GBS) through a systematic review of all published cases. METHODS: Pubmed, MEDLINE, EMBASE and Web of Science databases were searched from Jan 1960 to Sept 2014 with the search terms "human","enterovirus". "paralysis", "transverse myelitis " and "Guillain Barre Syndrome." Articles were included if they described one or more cases of suspected AFP with concurrent detection of NPEV by any method from any site. Articles were excluded if they studied only poliovirus, viruses other than enteroviruses, or only clinical presentations not consistent with AFP such as meningoencephalitis, hand-foot-mouth disease, and hemorrhagic conjunctivitis.

RESULTS: The search yielded 1618 articles of which 148 met the inclusion criteria.

The majority of reported NPEV AFP cases occurred outside North America, particularly in Asia. At least 50 different NPEV subtypes have been associated with AFP, with the most common being EV 71, Echovirus 11, Echovirus 6, Echovirus 9 and Coxsackie B3. EVD68, in the time frame of the study, was not commonly associated with AFP. Of those reports that detailed clinical followup, the majority of patients had residual paralysis. CONCLUSION: NPEV AFP cases have been predominantly reported in Asia, with EV 71 as the most commonly implicated subtype. EVD 68, though a recent concern, has not historically been associated with AFP. Standardized AFP surveillance programs may help identify the epidemiology and enterovirus subtypes related to AFP in North America.

\section{SP33}

\section{EVALUATION OF MATRIX ASSISTED LASER DESORPTION IONIZATION-TIME OF FLIGHT MASS SPECTROMETRY (MALDI-TOF MS) FOR YEAST IDENTIFICATION}

$\underline{\text { M Masud }}^{1}$, D Roscoe $^{1,2}$

\section{${ }^{1}$ University of British Columbia; ${ }^{2}$ Vancouver Coastal Health} Authority, Vancouver, BC

BACKGROUND: For most yeast species, antifungal susceptibility can be predicted based on yeast identification. MALDI-TOF was applied in this study to assess its accuracy when compared to traditional phenotypic methods for rapid yeast species identification.

METHODS: The microbiology database was used to identify yeast isolates recovered from blood and other sterile sites from January 2012 to December 2014. Isolates were sub-cultured twice to BAP (Oxoid, Basingstoke,UK), then to Brilliance Candida (Oxoid- Basingstoke, UK) for presumptive identification. Final identification was confirmed using microscopic and biochemical tests. MALDI-TOF MS (Bruker Daltonics, Bellerica, MA) was used for yeast species identification by spot extraction and later tube extraction to achieve reliable scores (>2.0 A). Discordant/ unreliable identifications were resolved by ITS sequencing.
RESULTS: This study tested 62 Candida and 10 Cryptococcus isolates. Concordant identification between phenotypic methods and MALDITOF was $100 \%$ to genus and $95.8 \%$ to species level (Table 1). Conventional methods failed to identify one isolate and ITS later confirmed MALDI-TOF identification.

CONCLUSIONS: Identification of yeast species by MALDI-TOF is accurate and reliable. The rapid identification of yeast by MALDI-TOF compared to conventional methods will substantially improve fungal diagnostics and choice of appropriate antifungal therapy.

\section{SP34}

XPERT MTB/RIF AS A SUPPLEMENTAL TEST TO ACID-FAST BACILLI SPUTUM MICROSCOPY IN THE DIAGNOSIS OF TUBERCULOSIS: POTENTIAL RESOURCE SAVINGS

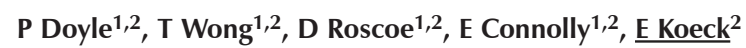

${ }^{1}$ Vancouver General Hospital; ${ }^{2}$ University of British Columbia,

Vancouver, BC

BACKGROUND: Diagnosis of pulmonary tuberculosis remains a challenge due to the low sensitivity of sputum microscopy and long culture turn-around times. Xpert MTB/RIF (Cepheid, Sunnyvale, CA) is a cartridge-based PCR assay which provides a higher sensitivity than microscopy and has exceptional turnaround time and ease-of-use. These advantages come with an increased per test cost. However, the increased cost of using Xpert MTB/RIF as a supplemental test to sputum microscopy may be offset by savings produced by better case detection.

METHODS: In this pilot study, retrospective data from1 large academic tertiary care centre and 2 community hospitals was fit into a theoretical model to determine whether routine implementation of Xpert MTB/RIF impacts resource utilization. A detailed cost-analysis was not part of this study. Data from a review of the microbiology specimen databases for the period January 12012 to December 312013 was used to determine whether airborne isolation time, infection control practitioner workload, and number of bronchoscopies performed would be impacted. Resource savings were calculated based on performing a single Xpert MTB/RIF assay per case, on the first sputum produced.

RESULTS: It was estimated that $36.2(95 \% \mathrm{CrI} 36.0$ - 36.6) days of airborne isolation, 24.5 (95\% CrI $21.2-27.0)$ hours of infection control practitioner time and 12.7 (95\% CrI $12.4-13.0)$ bronchoscopies could have potentially been avoided had Xpert MTB/RIF been performed on the first sputum in the 534 cases that were included in this study. The additional cost for 534 Xpert MTB/RIF cartridges at $\$ 49.98$ per test totaled $\$ 26,689$, exclusive of technologist time. Savings for bronchoscopies that were avoided were calculated using $\$ 1946$ per uncomplicated bronchoscopy for a savings of $\$ 25,532$ in bronchoscopy fees.

CONCLUSIONS: It appears from the results of this study that bronchoscopy savings alone are nearly enough to justify Xpert MTB/RIF implementation. These initial results suggest that further investigation is warranted with respect to the cost-effectiveness of routinely implementing Xpert MTB/RIF in clinical microbiology laboratories serving comparable hospitals in Canada.

TABLE 1

Summary of Maldi-TOF identification results

\begin{tabular}{|c|c|c|c|c|c|c|c|c|c|}
\hline Yeast Species (n) & $\begin{array}{l}\text { C. albicans } \\
(29)\end{array}$ & $\begin{array}{l}\text { C. glabrata } \\
\text { (12) }\end{array}$ & $\begin{array}{l}\text { C. parapsilo-sis } \\
\text { (12) }\end{array}$ & $\begin{array}{l}\text { C. tropicas } \\
(5)\end{array}$ & $\begin{array}{l}\text { C. lusitaniae } \\
\text { (3) }\end{array}$ & $\begin{array}{l}\text { C. krusei } \\
(2)\end{array}$ & $\begin{array}{l}\text { C. guillierm-ondii } \\
\text { (1) }\end{array}$ & $\begin{array}{l}\text { C. gattii } \\
\text { (3) }\end{array}$ & $\begin{array}{l}\text { C. neoforma-ns } \\
\text { (7) }\end{array}$ \\
\hline Phenotypic ID & 29 & 12 & 12 & 5 & 3 & 2 & No reliable id & 3 & 7 \\
\hline Maldi-tube id * & 3 & 1 & 2 & 2 & - & 1 & 1 & 2 & - \\
\hline ITS sequencing & - & - & 2 & - & - & & 1 & - & - \\
\hline
\end{tabular}

*Tube extraction performed only for isolates failed to achieve reliable id scores with spot extraction 


\section{SP35}

HOSPITALIZED INFLUENZA PATIENTS DURING 2013-2014: A COMPARISON OF ICU AND WARD TREATED PATIENTS INCLUDING ANTIMICROBIAL THERAPY, ADVERSE EVENTS, AND OUTCOMES

\section{A McFarlane, S Smith, W Sligl, L Saxinger}

University of Alberta, Edmonton, AB

OBJECTIVES: To describe the epidemiology of hospitalized patients with laboratory confirmed influenza infection during the 2013-2014 season at a single tertiary care hospital; to compare ICU and non-ICU patients; and to specifically describe antimicrobial therapy, outcomes and adverse events in the ICU cohort.

METHODS: Laboratory and epidemiologic data were collected at the University of Alberta Hospital through the Serious Outcomes Surveillance Network (SOS). Additional detailed diagnostic, clinical and outcome data were collected by retrospective chart review of the ICU cohort and stratified by diagnostic features association with bacterial infections.

RESULTS: Of 96 hospitalized influenza patients, 39 (41\%) required ICU care, 4 (4\%) required extracorporeal support, and $6(6 \%)$ patients died. H1N1 was the predominant strain in both ICU $(79 \%)$ and ward $(69 \%)$ patients. ICU patients were less likely to be vaccinated (2; $5 \%$ vs. $14 ; 25 \%)$; however vaccination status was unknown in a large number (49; $51 \%$ ) of patients. ICU patients were younger, with a higher BMI and comorbidity burden, had longer hospital stays, and higher rates of anti-bacterial use. Fourteen (36\%) ICU patients had bacterial infection on presentation. Twenty-one suspected or proven nosocomial infections were treated, including 9 presumed hospital/ventilator-acquired pneumonia ( 2 with antifungal therapy for Candida spp. on respiratory culture), 3 central venous catheter infections, 4 episodes of C. difficile infection and 3 cases of clinical sepsis with unknown source. In ICU patients the mean oseltamivir therapy duration was 8.8 days (range $0-19$ ), and initial antibiotic course was 9.2 days (range 0-18), with those classified as low likelihood of bacterial infection receiving 6.8 days (range 0-17). Directed antimicrobial treatment duration was 10 days (range 5-15) for C. difficile and 7.8 days (range 2-23) for other nosocomial infections. CONCLUSION: Patients admitted to the ICU with influenza infection were younger, had higher BMI and comorbidity burden, and lower vaccination rates. Regardless of risk for bacterial infection, antibiotic use was high in the ICU, with documented adverse outcomes such as C. difficile infection. Risk stratification for bacterial co-infection on admission may identify patients unlikely to benefit from antimicrobials thus minimizing unnecessary use.

\section{SP36}

CLINICAL SPECTRUM OF RHINOVIRUS INFECTIONS IN HOSPITALIZED CHILDREN IN ISRAEL - A SINGLE CENTER'S EXPERIENCE

G Pomeranz $^{1}$, E Mendelson ${ }^{2,3}$, M Mandelboim ${ }^{3}$

${ }^{1}$ Schneider Children's Medical Center of Israel, Petah Tikva; ${ }^{2}$ Tel Aviv University, Tel Aviv; ${ }^{3}$ Chaim Sheba Medical Center, Tel Hashomer, Ramat-Gan, Israel

BACKGROUND: Human Rhinoviruses are among the most common causes for acute respiratory illnesses (ARI), involving both the upper and lower respiratory tract (LRT). Clinical spectrum ranges from mild flu-like illness to severe respiratory compromise necessitating intensive care unit (ICU) admission. A known association exists between rhinoviral infections and childhood wheezing illnesses including asthma.

OBJECTIVES: To estimate for the first time the magnitude and characteristics of Rhinoviral infections in hospitalized patients in Israel.

METHODS: All the respiratory samples that were taken from hospitalized patients with ARI between July 2011 and June 2012, were tested for the presence of rhinovirus and other respiratory viruses by real time RT-PCR. Positive rhinovirus samples were tested further by Sequencing to classify the species. Demographic data, past and chronic illnesses, signs and symptoms of current illness, vital signs, laboratory tests, imaging and treatment were all documented from the patients' records.

RESULTS: 243 children tested positive for rhinovirus, which was perennially detected. In $44 \%$ a viral co-infection, most commonly adenovirus was present. $58 \%$ had significant co-morbid chronic illness (pulmonary, cardiac and prematurity). Common symptoms were cough (67\%), rhinorrhea (59\%), shortness of breath (44\%), and decreased appetite $(39 \%)$. Abnormal breathing sounds were found in $55 \%$ of the patients. $21 \%$ were admitted in ICU. Laboratory data showed monocytosis in $51 \%$, and hypercalcemia in $61 \%$ (findings not previously reported. Chest $\mathrm{x}$-ray was performed in $66 \%$ of the patients of them $59 \%$ had pathological findings. $63 \%$ received antibiotic treatment, $60 \%$ needed inhalations, $42 \%$ received systemic steroids. LRT involvement was identified in $75 \%$ of patients. There weren't any significant differences between rhinoviral species among these parameters.

CONCLUSION: Rhinovirus infections pose an extensive burden of respiratory illness in hospitalized children. Lower respiratory tract involvement is more common than that reported in the literature. Associated hypercalcemia is a new finding, as yet, unexplained.

\section{SP37 \\ PROSTHETIC JOINT INFECTIONS: PATTERNS OF PRACTICE FOR DIAGNOSIS, MANAGEMENT, AND OUTCOME}

D Harris, J De Beer, M Winemaker, D Petruccelli, D Mertz

McMaster University, Hamilton

OBJECTIVES: The IDSA guidelines in 2013 established a standard of management for prosthetic joint infections (PJI); however, implementation of the guideline tends to be challenging given the variability in clinical practice that has developed of the last centuries. We examine PJI cases at our centre comparing the outcomes of cases managed in accordance with the IDSA guidelines versus the outcomes of cases managed via another approach.

METHODS: A retrospective study of PJIs (NHSN/CDC criteria) from 2005 to 2012 at a tertiary care centre was conducted to determine clinical management variability and outcomes of deep and organ/space PJIs.

RESULTS: Of 8,505 hip and knee arthroplasty cases completed, 288 (3.4\%) were subsequently diagnosed with a PJI and $63(0.7 \%)$ cases were identified as deep or organ space PJI. Of these, 22 were knees (PJI rate of $0.2 \%$ ) and 41 were hips (PJI rate of $0.5 \%$ ). Eleven (17\%) had medical management only, and were excluded. Of those treated with a surgical approach, $28(44 \%)$ underwent irrigation and debridement (I\&D), 17 (27\%) had a one stage revision, and 7 (11\%) underwent two stage revision. In those treated according to the IDSA guideline, the risk for failure was significant lower (11/29 versus $8 / 11$; odds ratio $0.14,95 \%$ Confidence interval $0.03-0.64$ ). In the subgroup of those treated with I\&D, 1/19 and 5/9 failed, respectively (OR $0.04,0.004-0.49)$. Of the 17 patients who underwent a one stage revision, $1 / 7$ and $3 / 10$ failed, respectively (OR $0.39,0.03-4.80$ ). Of the 7 patients who underwent a two stage revision, 6 were treated according to the guidelines with one failure (16\% failure rate). The other patient had hardware left in place and was not classified due to the surgical complications.

CONCLUSIONS: We found significant variability in the management $\mathrm{PJIs}$, and that patients that were and could be managed in accordance with the 2013 IDSA guidelines had a significant lower failure rate, especially with respect to candidates for I\&D. This review provides evidence to support the use of the IDSA guidelines for treatment of PJIs.

\section{SP38}

URINARY BIOMARKERS IN PATIENTS WITH CYSTIC FIBROSIS E Vallieres ${ }^{1}$, G Parekh ${ }^{2}$, MM Tunney ${ }^{1}$, G Einarsson ${ }^{1}$, S McGrath ${ }^{1}$, L Mcllreavey ${ }^{1}$, E Johnston ${ }^{1}$, K Woolston ${ }^{2}$, D Downey ${ }^{1}$, P Davis ${ }^{2}$, JS Elborn 1

${ }^{1}$ Queen's University Belfast, Cystic Fibrosis \& Airways Microbiology Research Group, Belfast; ${ }^{2}$ Mologic Ltd, Bedford, UK

BACKGROUND: Urine biomarkers are potentially useful for early detection of lung inflammation or injury and may be a medium to detect pulmonary exacerbations in Cystic Fibrosis (CF). Optimal urinary biomarkers have not been identified and correlation with clinical outcomes has not been established. The aims of this pilot study were to 1) explore whether 24 biomarkers were detectable in the urine 2) determine if the levels of these biomarkers differ between CF patients and healthy individuals and 3) explore if biomarker levels correlate with disease severity data. 
METHODS: CF patients ( $\mathrm{n}=129$, aged 6-68 yr), enrolled at the paediatric or adult CF centres in Belfast, provided a total of 146 urine samples (135 when stable, 11 during exacerbation). Samples from healthy individuals $(\mathrm{n}=34$, aged $24-60 \mathrm{yr}$ ) were used as controls. Clinical and microbiology data were recorded. Samples were analysed with a combination of in-house (Mologic Ltd) and commercial assays (ELISA, lateral flow, protease substrate assay, zymography).

RESULTS: Every biomarker tested was detectable in urine and 9 were excreted at statistically different levels between patients and controls (Mann-Whitney, $P<0.05$ ). No strong and clinically significant correlation between biomarker levels and clinical data was highlighted. However, CF patients infected with methicillin-resistant Staphylococcus aureus $(\mathrm{n}=3)$ excreted higher levels of alpha-1 antitrypsin than those infected with Pseudomonas aeruginosa or Burkholderia cepacia complex $(P=0.038$ and $P=0.026)$. Although not statistically significant, a positive trend in desmosine, an amino acid related to elastin degradation, was found between stable and exacerbation time points (Wilcoxon signed-rank test, $P=0.069$ ). CONCLUSIONS: These results indicate the potential of urine biomarkers in CF. Further longitudinal studies are needed to determine whether fluctuation in biomarker levels correlates to clinical outcome and can be used to predict exacerbation onset.

This study was supported by the UK NHS NOCRI Translational Research Partnership \& US Ireland Partnership Grant. E.V. work is supported by AMMI Canada/Pfizer Post-Residency Fellowship and Cystic Fibrosis Canada.

\section{SP39}

\section{CARBON SOURCE MODULATES PARADOXICAL GROWTH} EFFECT OF CASPOFUNGIN IN CANDIDA ALBICANS

\section{A Delorme $^{1}$, RP Rennie ${ }^{1,2}$}

${ }^{1}$ University of Alberta; ${ }^{2}$ Provincial Laboratory for Public Health,

Edmonton, $\mathrm{AB}$

BACKGROUND: In vitro susceptibility testing of caspofungin by broth microdilution has revealed an unexplained paradoxical growth (PG) effect in which noticeable growth occurs at concentrations above the minimum inhibitory concentration (MIC) of susceptible isolates. The frequency of the PG effect is variable in different growth media, which vary most notably in the amount and types of carbon. We evaluated the effect of carbon source on the degree of PG in C. albicans to identify differences that may be applied to in vivo models and understand its clinical significance.

METHODS: C. albicans was grown in RPMI or minimal media containing $2 \%$ galactose, $2 \%$ lactate, and 3\% glycerol and with added caspofungin. Cultures were evaluated by time kill analysis. Samples collected over $72 \mathrm{hr}$ were evaluated for growth by colony forming units (CFU) and optical density at $600 \mathrm{~nm}\left(\mathrm{OD}_{600}\right)$.

RESULTS: C. albicans demonstrated a fungicidal effect at 0.075 and $2 \mathrm{mg} / \mathrm{L}$ caspofungin in all media. Growth at $16 \mathrm{mg} / \mathrm{L}$ caspofungin $(\mathrm{PG})$ was fungistatic by $\mathrm{CFU}$ determination. $\mathrm{By} \mathrm{OD}_{600}$ evaluation, C. albicans grown in fermentable sugars (RPMI, galactose) showed a statistically significant increase in PG compared to growth in non-fermentable sugars (lactate, glycerol). Large cell aggregates were seen in fermentable sugars, and unbudded yeast in non-fermentable sugars.

CONCLUSIONS: These data support a correlation between carbon source and the degree of PG in C. albicans, where the effect is significantly increased in fermentable carbon sources. There are significant physiological differences between yeast grown at PG and inhibitory caspofungin concentrations, Our observations suggest that further study of PG in different infection models may reveal what role PG plays in sustaining C. albicans in the different host niches where nutrients vary, despite apparent adequate antifungal therapy

\section{SP40}

COST-EFFECTIVENESS OF MALDI-TOF MS IN CLINICAL ANALYSIS OF URINE SPECIMENS IN NOVA SCOTIA H Al Sidairi ${ }^{1,2}$, T Hatchette ${ }^{1,2}$, D Haldane ${ }^{1,2}$, R Davidson ${ }^{1,2}$ ${ }^{1}$ Dalhousie University; ${ }^{2}$ Queen Elizabeth II Health Sciences Centre, Halifax, NS

BACKGROUND: Matrix Assisted Laser Desorption Ionization Time-ofFlight mass spectrometry (MALDI-TOF) is an automated microbial identification system revolutionizing bacterial identification. It is anticipated that the MALDI-TOF system will result in significant cost savings for the laboratory. We sought to evaluate the impact (financial and recovery) of using MALDI-TOF along with sheep blood agar (SBA) for urine cultures as an alternative to our current protocol utilizing Orientation agar.

METHODS: Prior to implementation, we retrospectively examined 3 years worth of urine culture data to determine our negative and positive culture rates and most commonly isolated pathogens. Prospectively, we continue to compare our isolation rates with historical data.

RESULTS: We process approximately 64,000 urine specimens per year of which $77 \%$ are negative. E. coli accounts for $55 \%$ of our positive isolates followed by K. pneumoniae, Enterobacter spp. and Enterococcus spp. (12\% and $11 \%$ respectively). When cost of each agar are considered (Orientation $\$ 0.62$, SBA $\$ 0.27$ ), a savings of over $\$ 17,000$ was realized for our negative cultures. We examined the additional cost of identification (SBA plus MALDI-TOF vs. Orientation agar plus biochemical) and realized another $\$ 1,000$ in savings. Our prospective study comparing SBA and MALDITOF to Orientation agar has yet to demonstrate a significant difference in the isolation rate or identification of any pathogen with one exception. We have noticed a small increase in "significant" Proteus spp., likely due to its spreading nature on SBA compared to Orientation agar.

CONCLUSION: The introduction of MALDI-TOF and the subsequent return to SBA for urine specimens have produced an annual cost savings of $\$ 18,000$ for our laboratory with no significant impact on isolation rate or identification of urinary tract pathogens.

\section{SP41}

\section{ROLE OF TYPE IV PILI IN THE CLOSTRIDIUM DIFFICILE PATHOGENIC PROGRAM}

\section{Martinez De La Pena ${ }^{1}$, Y Li ${ }^{1}$, G Maldarelli ${ }^{2}$, G Armstrong1,} M Donnenberg ${ }^{2}$

${ }^{1}$ University of Calgary, Faculty of Medicine, Calgary, AB; ${ }^{2}$ University of Maryland, School of Medicine, Baltimore, MD, USA Clostridium difficile is an anaerobic Gram-positive microorganism responsible for causing C. difficile infections, or CDI. CDI varies from mild diarrhoea to life-threatening inflammation of the colon. CDI has been associated with changes in the normal intestinal microflora mainly due to antibiotic therapy affecting especially hospitalized elderly people. The main virulence factors of Clostridium difficile are $\mathrm{Tcd} A$ and $\mathrm{TcdB}$ toxins. However, our recent discoveries demonstrated that $\mathrm{C}$. difficile can assemble Type 4 pili (T4P). T4P are filamentous structures expressed on the surface of bacteria and have been studied in many Gram-negative bacteria where they play an important role in colonization, adherence, biofilm formation and twitching motility. Assembly of T4P requires structural pilin proteins, extension and retraction ATPases, and pre-pilin peptidases, among others. Objective: To use a mutagenic approach to study the function of T4P in the C. difficile pathogenic program.

METHODS: TEM analysis was employed to investigate T4P expression by group II intron-targeted C. difficile mutant strains. Colonization was investigated using the antibiotic-treated mouse CDI model.

RESULTS: TEM analysis revealed that, unlike the wild-type parental strain, C. difficile pilA1 and pilU (pilin subunits), and pilD1 (pre-pilin peptidase) mutants were unable to express T4P. Moreover, initial results revealed no significant differences in the ability of the pilD1 mutant and wild-type strain to persist or cause illness in mice.

CONCLUSIONS: Our results suggest that, although T4P have been shown to play a role in colonization by Gram-negative bacteria, their role in C. difficile pathogenesis and persistence in mice, at least, remains to be determined. 


\section{SP42}

DETECTION AND CHARACTERIZATION OF A 2014 ESCHERICHIA COLI O157:H7 OUTBREAK IN ALBERTA BY MULTIPLE MOLECULAR METHODS INCLUDING WHOLE GENOME SEQUENCING

$\underline{\text { B Berenger }}^{1,2}$, C Berry ${ }^{4}$, C Nadon ${ }^{4}$, L Tschetter ${ }^{4}$, P Fack $^{5}$, S Delannoy ${ }^{5}$, V Li ${ }^{1}$, T Peterson ${ }^{4}$, L Chui ${ }^{1,3}$

${ }^{1}$ Alberta Provincial Laboratory for Public Health; ${ }^{2}$ University of Alberta Department of Medical Microbiology and Immunology; ${ }^{3}$ University of Alberta Department of Laboratory Medicine and Pathology, Edmonton, AB; ${ }^{4}$ Public Health Agency of Canada National Microbiology Laboratory, Winnipeg, MB; ${ }^{5}$ Food Safety Laboratory, Maisons-Alfort, France

OBJECTIVES: Over a three month period in 2014 a large E. coli O157:H7 outbreak occurred in the province of Alberta, Canada. We sought to characterize this outbreak using different molecular typing techniques including whole genome sequencing. A standardized method of whole genome analysis for determining strain relatedness has yet to be established, therefore in the context of this outbreak, we assessed the ability of core genome single nucleotide polymorphisms (SNPs) and K-mer phylogenies to discriminate between outbreak and sporadic E. coli O157:H7 isolates.

METHODS: We selected isolates received for molecular typing between July 10 and September 22, 2014 in addition to isolates selected from a 2012 beef-associated outbreak. Pulsed-field gel electrophoresis (PFGE), multiple-locus variable number tandem repeat analysis (MLVA), and shiga toxin (stx) subtyping were performed as per standardized protocols. Real-time PCR dynamic arrays were used to detect the presence or absence of 49 shiga toxin-producing E. coli virulence genes. Genome assembly and annotation were done using SPAdes and Prokka. Core genome SNP phylogenies were generated using the National Microbiology Laboratory bioinformatics SNVpyl pipeline.

RESULTS: 155 patient isolates were received: 10 from a small outbreak with indistinguishable PFGE/MLVA profiles and 111 from the 2014 outbreak comprising 23 with closely-related PFGE/MLVA profiles. All isolates from the 2014 outbreak were $s t x_{1 \mathrm{a}}$ and $s t x_{2 \mathrm{a}}$. No differences in virulence gene profiles were observed between the outbreak and sporadic isolates. In concordance with PFGE and MLVA, both core genome SNP and K-mer phylogenies clustered the 2014 and 2012 outbreaks as distinct events. CONCLUSIONS: Core SNP and K-mer phylogenies are appropriate methodologies to detect and investigate outbreaks of E. coli O157. Before whole genome analysis can be implemented for routine public health use, issues surrounding cost, technical expertise, software standardization, and data sharing/comparisons need to be addressed. Despite the unique characteristics of the 2014 E. coli O157 outbreak (ie. source, multitude of PFGE/MLVA profiles and size), no unique virulence gene profile could be identified.

\section{SP43}

\section{HEAT RESISTANT SHIGA TOXIN-PRODUCING ESCHERICHIA} COLI (STEC) FROM CLINICAL CASES IN ALBERTA

\section{$\mathrm{A} \mathrm{Ma}^{1}{ }^{1}$ LM Patterson-Fortin ${ }^{1}$, L Chui ${ }^{1,2}$}

${ }^{1}$ University of Alberta; ${ }^{2}$ Provincial Laboratory for Public Health, Edmonton, $A B$

OBJECTIVES: Foodborne disease due to the consumption of foods contaminated with Shiga toxin-producing E. coli (STEC) continues to be a global public health concern. With mechanical tenderization of meat in food processing plants, there is the potential for inoculation of STEC into deep, sterile tissue. Undercooked meat that does not reach temperatures capable of inactivating STEC further escalates the risk of disease. Environmental strains of E. coli and other Proteobacteria have demonstrated heat resistance but there is no evidence of heat resistant STEC involved in clinical disease.

METHODS: Heat resistance studies were performed on selected Alberta STEC isolates from 2006 to 2014. These isolates were inoculated in LuriaBertani (LB) broth with sodium chloride $(\mathrm{NaCl})$ concentrations of $1 \%, 2 \%$, and $4 \%$, reflective of $\mathrm{NaCl}$ concentrations used in meat preservation. Recovered cells were exposed to $60^{\circ} \mathrm{C}$ heat treatment in a water bath for 15 , 30,45 , and 60 minutes. Survival was quantified by colony enumeration of heat treated isolates on LB agar and those yielding 50 or more colonies were considered heat resistant. Protein gel electrophoresis was performed on all heat resistant isolates and compared to heat sensitive isolates. Isolates were exposed to $37^{\circ} \mathrm{C}$ and $60^{\circ} \mathrm{C}$ heat treatment for 60 minutes prior to protein denaturation to investigate differences in protein expression between isolates and whether expression was inducible or constitutive.

RESULTS: Heat resistance was confirmed in three clinical STEC isolates. Serotypes of the these STEC isolates were ONT:H25, O11:H30, and $\mathrm{O} 157: \mathrm{H} 3$. All positive isolates survived $60^{\circ} \mathrm{C}$ heat treatment for greater than 60 minutes. Serotype O11:H30 displayed greater heat resistance in $1 \% \mathrm{NaCl}$ whereas $4 \% \mathrm{NaCl}$ was favoured for serotypes ONT:H30 and O157:H3. Upon protein gel electrophoresis, evidence suggests that positive isolates express additional protein bands at $35 \mathrm{kda}$ and $110 \mathrm{kda}$ that were visualized both in isolates exposed to $37^{\circ} \mathrm{C}$ and $60^{\circ} \mathrm{C}$ heat shock.

CONCLUSIONS: Heat resistant STEC involved in clinical infection in Alberta was confirmed by in vivo studies. Positive isolates displayed the ability to survive $60^{\circ} \mathrm{C}$ heat treatment for greater than 60 minutes. The presence of heat resistance associated with STEC plays an important role in food safety, especially in determining the duration of cooking. temperatures.

\section{SP44}

\section{THE USE OF ANTIBIOTICS IN THE TREATMENT OF SKIN AND SOFT TISSUE INFECTIONS IN SELECTED FIRST NATIONS COMMUNITIES}

D Jeong ${ }^{1}$, Y Schreiber ${ }^{2,3}$, M Tyndall1,2,3

${ }^{1}$ University of Ottawa, ${ }^{2}$ The Ottawa Hospital; ${ }^{3}$ Ottawa Hospital

Research Institute, Ottawa, ON

OBJECTIVE: The rate of MRSA infection has been rising in Canada with First Nations (FN) populations at higher-risk. To date, the prevalence of MRSA and the antibiotic susceptibility patterns have not been assessed by a community-based study at the national level. The objective of this study was to describe the local epidemiology of skin and soft tissue infections (SSTIs) in FN communities and to describe antibiotic use.

METHODS: This study was a retrospective chart review at 12 nursing stations in FN communities across 5 provinces. People over 18 who visited the nursing station in the previous 12 months were included. Each encounter that resulted in an antibiotic prescription during the study period was captured as a "case". Demographic information, indication for therapy, details of antibiotic use, microbiological data and treatment appropriateness were assessed.

RESULTS: In total, 372 charts were reviewed, 459 cases were recorded and 137 of these represented SSTI. Over $80 \%$ of the SSTI patient population were under 50 . Based on the findings of the study, the prevalence of SSTI among the FN communities in 2012-2013 was estimated at 37\%. 55 cases were identified as MRSA infections by culture or by a history of MRSA colonization. The prevalence of MRSA in SSTI was $40.1 \%$. Most MRSA isolates in this study were susceptible to clindamycin and co-trimoxazole (90.5\% and $95.2 \%$ ), with only $29 \%$ susceptible to erythromycin. The majority of SSTIs were purulent infections and wounds. Wound cultures were performed in only $29 \%$. Thirty percent of antibiotic prescriptions were judged to be inappropriate. Most (72\%) infections were treated orally. Higher rates of SSTIs were seen in communities where overcrowding and poor access to running water are more prevalent.

CONCLUSIONS: SSTIs, specifically due to MRSA, are common in FN communities in Canada. Appropriate prevention and treatment of SSTIs in these communities require a better understanding of the unique challenges faced by these communities as well as establishment of surveillance systems and antimicrobial monitoring programs. 


\section{SP45}

THE ROLE OF INTER-HOSPITAL SAMPLE TRANSPORT IN TOTAL ASSAY TIME FOR MICROBIOLOGICAL SCREENING TESTS

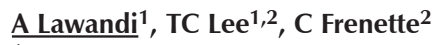

${ }^{1}$ Department of Medicine, Division of Internal Medicine, McGill University Health Centre; ${ }^{2}$ Department of Medicine, Division of Infectious Diseases, McGill University Health Centre, Montreal, QC BACKGROUND: McGill University Health Centre uses PCR to screen all admitted medical patients for MRSA and VRE, and if symptomatic, for respiratory viruses. PCR can yield same day results, which helps to optimize isolation precautions and patient flow. However, with planned centralization of laboratories, such as in Quebec, there is concern that inter-hospital transport of samples may retard results, impacting patient flow.

METHODS: The Royal Victoria Hospital sends its screening samples to the Montreal General Hospital, $1.7 \mathrm{~km}$ away. MRSA, VRE and respiratory virus PCR screenings for admitted medical patients at the Royal Victoria Hospital were analysed to determine the time required for each step of the screening process and their contribution to total assay time.

RESULTS: Data from 100 admitted to internal medicine was recorded and pertinent results are summarized in Table 1. Importantly, the time between sample collection and receipt in lab increased significantly after 15:00 with the time spent "in transport" making up a sizable proportion of the overall analytic time. This led to a substantial delay in result availability and potentially caused prolongation of unnecessary isolation precautions and delays in the transfer of patients from the emergency room to the inpatient units.

CONCLUSIONS: The transportation of specimens between sites contributes significantly to the overall turn-around time and as laboratory technical time decreases, the importance of this contribution increases. It thus represents an important barrier to the timely completion of screening assays as even short inter-hospital transportation may be a rate limiting step in the timely completion of assays. Such delays ought to be considered when planning for a centralized laboratory system such as Quebec's Optilab project.

TABLE 1

Test performance characteristics and contribution of transport time to assay time

\begin{tabular}{llll}
\hline & MRSA & VRE & Resp. Virus \\
\hline Positive Rate (\%) & 6.7 & 11.0 & 40.4 \\
$\begin{array}{c}\text { Mean Total Turn-around } \\
\quad \text { Time (hours) }\end{array}$ & $44.2( \pm 22.2)$ & $51.1( \pm 23.8)$ & $18.2( \pm 10.8)$ \\
$\begin{array}{c}\text { Average Proportion of Time } \\
\text { spent "in transport" (\%) }\end{array}$ & 28 & 14 & 60 \\
\hline
\end{tabular}

\section{SP46}

INVESTIGATION OF MATRIX ASSISTED LASER DESORPTION/IONISATION TIME-OF-FLIGHT MASS SPECTROMETRY (MALDI-TOF MS) FOR DETECTION OF CLOSTRIDIUM DIFFICILE TOXIN A AND B FROM STOOL SAMPLES

$\underline{\text { C Lam }}{ }^{1}$, A McClean ${ }^{1}$, E Koeck ${ }^{1}$, L Ang² ${ }^{2}$ P Naidu ${ }^{3}$, K Donkor ${ }^{4}$, K Wagner ${ }^{1}$, N Cheeptham ${ }^{1}$

${ }^{1}$ Department of Biological Sciences, Faculty of Science, Thompson Rivers University, Kamloops, BC; ${ }^{2}$ Microbiology Laboratory, Royal University Hospital, Saskatoon, SK; ${ }^{3}$ Provincial Laboratory for Public Health, University of Alberta, Edmonton, AB; ${ }^{4}$ Department of Physical Sciences, Faculty of Science, Thompson Rivers University, Kamloops, BC

OBJECTIVE: This study aims to investigate the use of MALDI to directly detect $C$. difficile toxin A and B from clinical stool samples.

METHODS: Stool samples sent to Royal Inland Hospital that were tested positive for C. difficile by glutamate dehydrogenase and toxin were diluted 10 -fold in deionized water or phosphate buffered saline and vortexed to create a relatively homogeneous suspension. Samples were then centrifuged and the pellet removed. Proteins in the supernatant were precipitated with acetonitrile or ammonium sulfate and the solution was centrifuged again. The pellet was then resuspended in deionized water or TA30 and spotted on a MALDI target plate with a sinnapinic acid or SDHB matrix co-crystalized. RESULTS: MALDI analysis showed no difference between samples diluted in deionized water and those diluted in phosphate buffered saline. Protein precipitation with acetonitrile produced higher quality spectra than protein precipitation with ammonium sulfate. Sample co-crystalization with sinnapinic acid provided higher quality spectra than SDHB and no difference in spectra quality was seen between samples resuspended in deionized water and those resuspended in TA30. MALDI analysis showed a peak at $56 \mathrm{kDa}$ in four samples. No peaks were seen in the $63 \mathrm{kDa}$ range in any of the samples. It is speculated that this $56 \mathrm{kDa}$ protein is a metabolite of C. difficile toxins. Autocleavage of a commercially known toxin A also failed to show the expected peak at $63 \mathrm{kDA}$

CONCLUSION: These findings show that we were unable to use MALDI to directly detect $\mathrm{C}$. difficile toxin $\mathrm{A}$ and $\mathrm{B}$ from crude stool protein extracts. Further studies would be required to ascertain the possibility of using this technological tool to detect $\mathrm{C}$. difficile toxin as an alternative method of diagnosis to the tests currently available. Although inconclusive, this study is a starting point for the use of MALDI as a diagnostic tool in a clinical setting.

\section{SP47}

\section{CUMULATIVE ANTIMICROBIAL SUSCEPTIBILITY DATA FROM ICUS AT ONE INSTITUTION: SHOULD DATA BE COMBINED?}

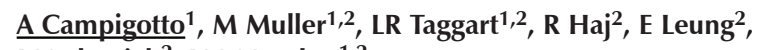
J Nadarajah'2, LM Matukas ${ }^{1,2}$

${ }^{1}$ University of Toronto; ${ }^{2}$ St Michael's Hospital, Toronto, ON

BACKGROUND: Cumulative susceptibility test data (CSTD) is used to guide empiric antimicrobial therapy and track resistance trends. In order to achieve adequate sample sizes when analysing intensive care unit (ICU) data we explored the differences between combining data from $>1$ ICU versus combining data over longer periods of time for each ICU at St Michael's Hospital.

METHODS: We used data from 2009 to 2014 to present annual CSTD using 2 different methods: 1) combining data from medical surgical ICU (MSICU) and trauma-neuro ICU (TNICU) or 2) using a 2 year rolling average with results for each ICU analysed separately. Ciprofloxacin susceptibility results for P.aeruginosa were representative of the differences seen in data analysis.

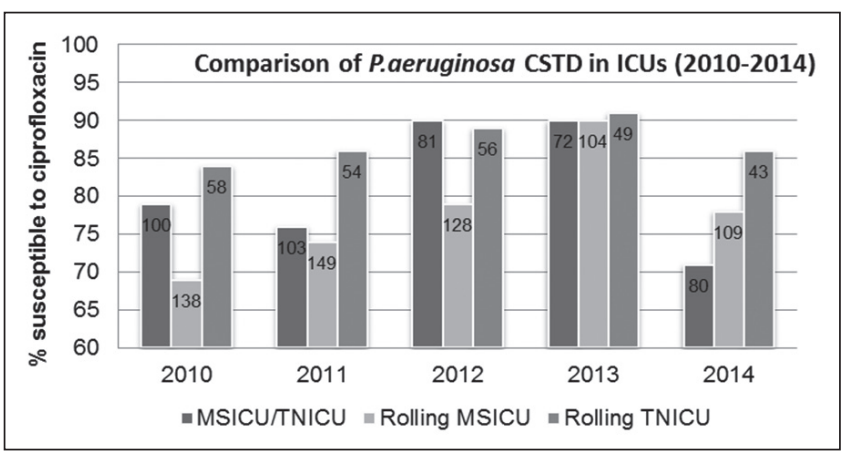

RESULTS: Values within the bars represent the number of isolates.

CONCLUSIONS: Differences in susceptibilities were larger between ICUs than they were year over year. When ICUs differ in their microbial ecology, combining data from $>1$ ICU is not appropriate and may result in inappropriate empiric therapy in both ICUs. Instead, combining data from $>1$ year can ensure appropriate sample size while providing accurate susceptibility results. 


\section{SP48}

INFECTIOUS COMPLICATIONS AFTER CENTRAL VENOUS CATHETER REPAIR IN PEDIATRIC PATIENTS

G Shah, B Lee, J Durand, N Gartner, S Forgie

University of Alberta, Edmonton, $\mathrm{AB}$

OBJECTIVES: Children with long-term central venous catheters (CVC) often develop cracks/breaks in their CVC. In order to delay surgery for new CVC and preserve venous sites, repair of broken CVC using aseptic technique has become more common. There is little published data on infectious complications after CVC repair. This study investigated the association between line repair and CVC blood stream infection (CVC BSI).

METHODS: A retrospective chart review (2010-2014) of all children with CVC repair was completed. Demographics, type of catheter, underlying diagnosis, antibiotic exposure, total parenteral nutrition (TPN), immune status, organisms isolated and resistance patterns, other complications and date of CVC removal after repair was collected. Bloodstream infection data was obtained from the Infection Prevention and Control database (prospectively collected data using NHSN Definitions for CVC BSI) and laboratory database. Primary outcome was the rate of CVC BSI post repair and the secondary outcomes were risk factors affecting the incidence of CVC BSI post repair.

RESULTS: Ten patients were included (age range 2-16 years, 7 males): Nine had malignancies (3- AML, 1-ALL, 1- neuroblastoma, 1- ependymoma, 2- medulloblastoma and 1- Burkitt's Lymphoma), one had intestinal atresia and short gut syndrome. Nine were frequently neutropenic, and 4 received continuous or intermittent TPN. In the pre-repair (30 day) period $5 / 10$ were on broad spectrum antibiotics. $0 / 10$ were on antibiotics 30 days post repair. Six had a positive cultures- 3 were positive in the prerepair (30 days) period (E. coli, viridans group streptococci and $\mathrm{M}$. catarrhalis), and 3 were positive $>30$ days after CVC repair (S. maltophilia, Leuconostoc sp. and Diphtheroids). Eight of the repairs were successful (i.e. line retained $>30$ days). None of the patients had clinical deterioration within 30 days of line repair.

CONCLUSIONS: It is difficult to draw conclusions about the bloodstream infection risk after CVC repair in children. However, for this case series, there were no CVC BSI in the immediate post repair period, and $80 \%$ of the children were able to keep their lines for $>30$ days post repair. A prospective study with larger enrolment would be more helpful in predicting the short and long-term complications of CVC repair.

\section{SP49 \\ ACUTE ODONTOGENIC INFECTION MANAGEMENT IN THE OPAT CLINIC SETTING: A QUALITY IMPROVEMENT STUDY IN CALGARY, ALBERTA \\ W] Connors, MD Parkins, DL Holton \\ University of Calgary, Calgary, AB \\ BACKGROUND: Acute ondontogenic infections (AOI) account for an estimated $60 \%$ of all non-traumatic dental emergencies and over six hun- dred thousand visits to Canadian emergency departments each year. The objectives of this study were to quantify and characterize AOI amongst adults presenting to acute medical care and managed through a city-wide out-patient antibiotic therapy program (OPAT). Secondary objectives were to estimate the healthcare costs of AOI management and develop an integrated care pathway. \\ METHODS: Adult acute medical care and OPAT AOI referrals were quan- tified using diagnostics codes in a regional reporting system. OPAT referrals consenting to participation in the study completed a questionnaire and had their OPAT medical records reviewed upon completion of care. Through collaboration with local medical and dental services a standardized medical template and referral pathway was developed. The study was conducted in Calgary, Alberta, between February and June, 2014 \\ RESULTS: Of 704 adult individuals presenting to Calgary area acute care facilities with AOI during the study period 343 [49\%] were referred to OPAT [5.5\% of all OPAT referrals]. A total of 110 [32\%] individuals were included in the study (women 60 [54\%], mean age 44 years [range 18-96]). The major- ity of individuals with AOI were smokers (53 [53\%]), had dental insurance (70 [65\%]), had seen a dentist in the past six months (70 [65\%]) and}

reported prior dental infections (84 [77\%]). Thirty-nine [46\%] reported current $\mathrm{AOI}$ as a recurrence. Median monthly pre-tax household income was $\$ 4800$ with 16 (19\%) of those responding falling below the Canadian LowIncome Cut-off. Median length of parenteral antibiotic therapy was 3 days (range 1-13, IQR 2) resulting in an average 15-day total course of antibiotics (range 0-35, IQR 4). Estimated total cost saving of OPAT care compared with hospitalization for intravenous portion of antibiotics was $\$ 597,434$.

CONCLUSIONS: AOI represent a major preventable cause of recurrent morbidity resulting in extended antibiotics exposures and accounting for substantial healthcare costs. OPAT appears to provide a cost-effective model for initial AOI management. Integrated care pathways for AOI may facilitate collaboration with dental services allowing timely provision of definitive management and prevent reoccurrence.

\section{INNOVATION ACADEMY POSTER PRESENTATIONS}

\section{IA01 \\ REAL-TIME COMPUTER-GENERATED ALERTS TO SELECT INTERVENTIONS AND OPTIMIZE AN ANTIMICROBIAL STEWARDSHIP PROGRAM}

P Dolce $^{1}$, A Boivin ${ }^{2}$, L Bergeron ${ }^{3}$, E Labrecque ${ }^{3}$, H Bernatchez ${ }^{1}$ ${ }^{1}$ Department of Medical Microbiology and Infectious Diseases, CSSS Rimouski-Neigette (CSSSRN); ${ }^{2}$ Department of Biology, Université du Québec à Rimouski, ${ }^{3}$ Department of Pharmacy, CSSSRN, Rimouski, QC

OBJECTIVES: Implementation of antimicrobial stewardship program (ASP) is a challenge, with limited physician and pharmacist time. A multi-disciplinary approach (clinical pharmacists, medical microbiologists, infectious diseases, information technology) was undertaken to integrate information technology in our ASP, with alerts to tract potential interventions.

METHODS: CSSSRN is a tertiary regional hospital with 230 acute-care beds. A software "Antibiokos" was developed to enable our ASP team to tract interventions, using real-time data from interfaces with pharmacy, microbiology laboratory and admission-discharge-transfer. Electronic algorithms included core elements of ASP such as: time-sensitive stop orders ( 3 days for IV, 7 days for oral), overlapping spectra, switch from intravenous to oral, drug optimization according to culture results, formulary restriction and pharmacokinetics (PK). Real-time metrics (DDD, DOT) were provided, with measurements of outcome such as Clostridium difficileassociated diarrhea. Implementation was performed in November 2014. OUTCOMES: During the first 2 months, a total of 3292 DOT were observed among inpatients, and 306 electronic alerts were generated in 179 patients. The alerts were time-sensitive stop orders $73 \%$, optimization according to culture $8 \%$, PK $8 \%$, switch IV to oral $4 \%$, overlapping spectra $3 \%$, other $4 \%$. A total of 97 interventions were done in 71 patients, mainly on Pip-Tazo 19\%, Quinolones 17\%, Cephalosporins 17\%, Carbapenems $14 \%$, Macrolides $8 \%$, Antifungals 4\%, Antivirals 3\%. Suggestions were: no change $55 \%$, change $24 \%$, stop $16 \%$ and addition $5 \%$. High acceptance rate of suggestions (95\%) was observed from attending physicians. The software provided significant time-reduction with estimates : $>95 \%$ to get the metrics (DDD or DOT), and $>50 \%$ to target and perform ASP interventions. More completed data will be presented at the conference.

\section{IA02 \\ INCLUSION OF RHINOVIRUS/ENTEROVIRUS IN ROUTINE RESPIRATORY TESTING ENABLED TIMELY DETECTION OF ENTEROVIRUS D68 IN THE HAMILTON AREA}

C Rutherford ${ }^{1}$, K Luinstra ${ }^{2}, M_{\text {Smieja }}{ }^{1,2}$

${ }^{1}$ Hamilton Regional Laboratory Medicine Program; ${ }^{2}$ McMaster University Department of Pathology and Molecular Medicine, Hamilton, ON

OBJECTIVE: In 2012, the decision was made to include rhinovirus/ enterovirus detection in a lab developed multiplex PCR that detects eight respiratory viruses and an internal control and has been in use since 
November 2010. Routine use of the assay with rhinovirus/enterovirus started in September 2013.

METHODS: The multiplex assay runs in two tubes. The first tube detects Influenza A and B, RSV and the internal control. The second tube tests for parainfluenza 1, 2 and 3, metapneumovirus and adenovirus. The assay runs on the Rotorgene (Qiagen) real time thermal cyclers which have five detection channels. The crimson channel in tube one was available for rhinovirus/enterovirus detection.

Primers and a probe were designed using sequences from GENBANK and aligned using Clustal Omega. Specificity was checked using NCBI BLAST. The final design has two forward rhinovirus primers and one forward enterovirus primer with one reverse primer and a probe that cover both rhinovirus and enterovirus. Detection of rhinovirus A, B and C was tested with previously sequenced patient samples. Detection of enterovirus was tested using stock strains of echovirus, enterovirus and coxsackie $\mathrm{A}$ and $\mathrm{B}$ viruses.

Reaction efficiency and sensitivity were compared by running the multiplex assay at the same time as uniplex assays for each target in the tube. Assay sensitivity was considered acceptable if the crossing threshold $(\mathrm{Ct})$ of the multiplex was within $2 \mathrm{Cts}$ of the uniplex Ct. The LOD of the assay for rhinovirus/enterovirus using enterovirus-D68 as the target is 306 copies/mL. Detection levels for Infuenza A and B and RSV were not compromised. External performance assessment challenges are obtained from IQMH, NML and CAP. Data on positivity rates for each virus are tabulated weekly. The expanded assay has been performed 1-3 times daily by the clinical laboratory since September 2013.

OUTCOMES: Since September 2013 we have tested 11,930 respiratory specimens. Of these 1777 (14.8\%) were positive for rhinovirus/enterovirus , the most commonly detected pathogen in our population. From August to October 2014 a spike in rhinovirus/enterovirus detection (661 positives) was noted concurrent with reports of enterovirus-D68 (EV-D68) in the USA. An EV-D68 specific assay was quickly developed in September and validated against sequencing results. Testing for EV-D68 was performed within 24 hours of obtaining a positive rhinovirus/enterovirus result. Of 647 rhinovirus/enterovirus positive samples tested in the specific assay, 183 (28.3\%) were EV-D68 positive. The addition of rhinovirus/enterovirus to the multiplex assay was welcomed by the clients we serve, especially the pediatric infectious disease physicians and allowed us to react to the emergence of enterovirus-D68 in a timely manner. The addition of the EV-D68 specific assay to the testing protocol was very important to Infection Control at the McMaster Children's Hospital allowing them to cohort infected children and prevent nosocomial spread of enterovirus-D68.

\section{IA03}

\section{PREVENTING C. DIFFICILE INFECTION BY SCREENING HOSPITAL ADMISSIONS FOR C. DIFFICILE}

YM Murad $^{1}{ }^{1}{ }^{2}$, J Perez ${ }^{1}$, R Nokhbeh ${ }^{1,2}$, G Ybazeta ${ }^{1}$, F Diaz-Mitoma ${ }^{1,2}$ ${ }^{1}$ Advanced Medical Research Institute of Canada; ${ }^{2}$ Northern Ontario School of Medicine, Sudbury, ON, Canada

BACKGROUND: CDC defines C. difficile infection (CDI) as the number one threat to public health. CDI prevalence in most healthcare facilities, suggest that current interventions to prevent or decrease CDI, including treatment of established infection and infection control to mitigate spread of infection are inadequate. Several studies show that between $10-15 \%$ of admitted patients are C. difficile carriers. If carriers develop diarrhea, they may erroneously be labeled "healthcare-associated" CDI. Currently, there are no standards to manage $\mathrm{C}$. difficile carriers. A more proactive strategy for managing and preventing CDI including hospitalized patients who are asymptomatic carriers needs to be developed.

HYPOTHESIS: The identification and management of asymptomatic C. difficile carriers on hospital admission may prevent disease and transmission of CDI. Moreover, early intervention in high-risk carriers will improve clinical outcome and decrease the burden of healthcare-acquired CDI.

METHODS: Two general medical wards were targeted for this C. difficilescreening program. These medical wards were chosen due to the higher prevalence of CDI than other wards. We used a PCR based test in stool for detection of C. difficile carriers. We interviewed 826 newly admitted patients of whom 376 patients consented for stool and blood collection in a period of approximately 6 months.
RESULTS: Only 160 out of the 376 consented patients had an evaluable stool sample for PCR testing. We identified over $10 \%$ of tested patients as asymptomatic carriers of C. difficile (17patients tested positive for C. difficile). Results long with explanation of the significance of C. difficile carriage were communicated to the attending physician. Identifying C. difficile carriers among the most vulnerable patients may offer alternatives to avoid iatrogenic CDI.

CONCLUSIONS: In this preliminary study, we tested and identified C. difficile carriers upon admission to the hospital among a selected group of patients at high risk of developing CDI (oncology patients). Our goal is to extend the screening to all high risk hospital admission for C. difficile to identify carriers at risk of severe disease. Identification of these high-risk patients, combined with hand washing, a strict antimicrobial stewardship program and careful environmental decontamination may help in reducing the rates of CDI significantly.

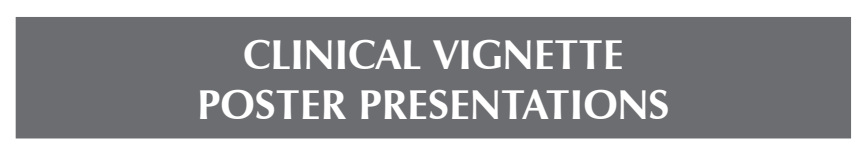

\section{CV01 \\ ADENOVIRUS INTERSTITIAL NEPHRITIS IN A PATIENT}

$\underline{\text { S Ala }}$

Miami Valley Hospital; Department of Medicine, Wright State University, Dayton, OH, USA

We present the case of a 65 -year-old woman with a history of diabetes mellitus, a CABG in 1996 and end-stage renal failure leading to renal transplant in June 2005. She was well until November 2, 2013 when she developed cough and a decrease in oral intake. She was prescribed Doxycycline for PCP with no improvement. November 8, 2013, she developed weakness and fever and fell. She was admitted for dehydration and hyponatremia, but despite hydration, her creatinine did not return to baseline. All the investigations were unremarkable except for her kidney biopsy, which revealed acute adenoviral (ADV) nephritis (interstitial nephritis). Her ADV PCR was positive at 64,500 copies (blood) and $10,000,000$ copies/mL (urine). We reviewed the case management and provide a brief review of the literature.

\section{CV02}

A CASE OF CAPNOCYTOPHAGA CANIMORSUS MENINGITIS G Patriquin ${ }^{1}$, S McNeil ${ }^{2}$

${ }^{1}$ Department of Medicine; ${ }^{2}$ Division of Infectious Diseases, Department of Medicine, Dalhousie University, Halifax, NS

The case is that of a 57-year-old man, presenting with clinical signs and symptoms of bacterial meningitis and treated empirically with ceftriaxone and vancomycin. Though his CSF profile was consistent with bacterial meningitis (WBC of $1329 \times 10^{6} / \mathrm{L}$ ( $90 \%$ neutrophils), glucose of $2.6 \mathrm{mmol} / \mathrm{L}$, protein of $2.06 \mathrm{~g} / \mathrm{L}$ ), and the CSF Gram stain demonstrated rare Gram-negative rods, the organism failed to grow in culture, leaving us puzzled as to the etiology of his illness.

After his eighth day of hospitalization, he was feeling well enough to return home for recovery and ongoing therapy with ceftriaxone. After discharge, the result of a $16 \mathrm{~S}$ ribosomal RNA sequencing of his CSF revealed the etiology of his meningitis as Capnocytophaga canimorsus. Only on subsequent direct questioning after the diagnosis did the patient endorse both a bite from his household dog 1-2 days prior to his illness, and excessive alcohol intake (of approximately 24 bottles of beer per week), both risk factors for systemic disease due to C. canimorsus.

The patient was reassessed in the Infectious Diseases ambulatory clinic two weeks after discharge, and had fully recovered, except for mild hearing loss, after 21 days of ceftriaxone. Further investigation of his liver and spleen health revealed no apparent impairments. It was recommended that he undergo vaccination as would a hyposplenic patient. 
CV03

WITHDRAWN

CV04

ATYPICAL CASE OF ATYPICAL PNEUMONIA:

DISSEMINATED MYCOPLASMA HOMINIS INFECTION IN A DOUBLE LUNG TRANSPLANT RECIPIENT

A Mah ${ }^{1}$, I Wong $^{2}$, L Hoang ${ }^{3}$, A Stefanovic ${ }^{2}$

${ }^{1}$ Division of Infectious Diseases; ${ }^{2}$ Division of Medical Microbiology, Department of Pathology \& Laboratory Medicine, University of British Columbia; ${ }^{3}$ BCCDC Public Health Microbiology \& Reference Laboratory, Provincial Health Services Authority, Vancouver, BC

We present the case of a 67-year-old man who underwent a double lung transplant for end stage interstitial lung disease. On post-operative day (POD) 6, while still intubated, the patient developed a fever and increasing oxygen requirements. There were no other infectious symptoms noted. His past medical history was otherwise significant for coronary artery disease with a prior NSTEMI in 2001. As well, the patient had a history of gastroesophageal reflux disease and hepatic steatosis. Pertinent medications included tacrolimus $2.5 \mathrm{mg}$ daily, MMF $1500 \mathrm{mg}$ BID and prednisone $60 \mathrm{mg}$ daily. He had been on meropenem perioperatively and continued postoperatively. He was also on fluconazole for a BAL growing scant C. albicans as well as septra and acyclovir prophylaxis. Investigations were significant for an elevated WBC count 14.6 and mildly elevated LDH 352 . Serial chest $\mathrm{x}$-rays showed fluctuating areas of bibasilar atelectasis/consolidation with bilateral pleural effusions. Multiple microbiologic specimens were sent including BAL and chest tube site swabs as well as blood cultures all of which were negative. On POD 6, a BAL specimen submitted on POD 3 showed growth on blood agar plates. The original Gram stain from the specimen was $2+\mathrm{PMN}$ with no organisms seen. Attempts to perform a Gram stain on the isolate growing on BAP did not reveal any organisms. Given the suspicion of an atypical respiratory pathogen, the isolate was sent to the reference laboratory for identification and azithromycin and moxifloxacin were added to the patient's treatment regimen. The original isolate was identified as Mycoplasma hominis by $16 \mathrm{~S}$ PCR. This organism was also isolated from several repeat BAL specimens, swabs from the patient's chest tube sites and from a culture of the patient's internal jugular catheter tip, although all blood cultures failed to grow. The patient was diagnosed with disseminated Mycoplasma hominis infection with a respiratory focus occurring post-double lung transplant. 


\section{A}

Abdesselam K ....................L04

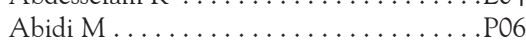

Adam E ..................... K02

Adam HJ ........ D02,L02,L03 L05,SP29

Adomako K . . ..................E06

Adumattah A ................SP28

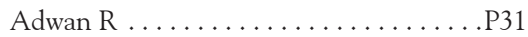

Al-Busaidi I . . . . . . . . . . . . . SP27

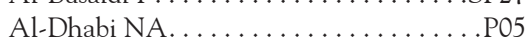

Al-Mansouri W . . ................... 31

Al-Massarani S. . . . . . . . . . . . . . . . P01

Al Musayeib N ..................P01

Al Mutawa F . . . . . . . . . . . . . . . . K03

Al Sidairi $H \ldots \ldots \ldots \ldots \ldots . \ldots . \ldots . \ldots 40$

Ala S ................................

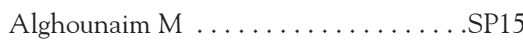

Allan D .......................P23

Allison D .................... HO2

Ames L.........................P01

Andonova M . . . . . . . . . . . . . . . . F04

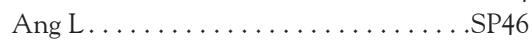

Archibald C . . .................. L04

Armstrong G . . . . . . . . . . . . . SP19,SP41

Ash $\mathrm{T} \ldots \ldots \ldots \ldots \ldots \ldots \ldots \ldots . . \ldots \ldots 2$

Asztalos E ...................E03

\section{B}

Ba Taher H. . . . . . . . . . . . . . . . . SP30

Bai A ................... P09,SP09

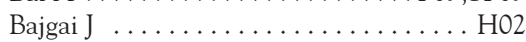

Balakumar S..................P27

Barber D. . . . . . . . . . . . . . . . SP19

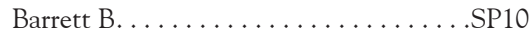

Barth C . . . . .................. A02

Barton M ....................E03

Bass $\mathrm{S} \ldots \ldots \ldots \ldots \ldots \ldots \ldots \ldots \ldots$. . . . . . . . . . . .

Baxter M . . . . . . . . D02,L02,L03,L05,SP29

Beaudry G . ................. I03

Bekal S ................... I03,105

Bell C................... P09,SP09

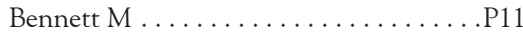

Berenger B ..................SP42

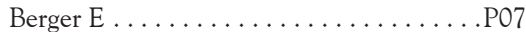

Bergeron L . . . . . . . . . . . . . . . IA01, SP20

Bernard K . . . . . . . . . . . . . . . . B04

Bernatchez H . . . . . . . . . . . . . . . IAO1

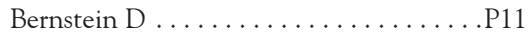

Berry C . . . . . . . . . . . I03,I05,SP42

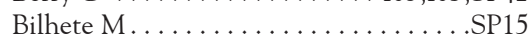

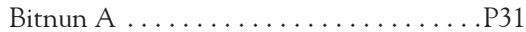

Blanchard A. . . . . . . . . . . . . . . . . . .

Blondel-Hill E . . . . . . . . . . . . . . . . . D04

Boikos C ................ F03,SP18

Boivin A .......................

Booth T . . . . . . .............F05

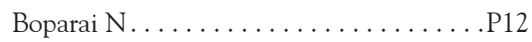

Borgundvaag B..............SP26

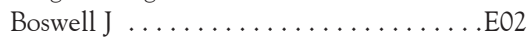

Boucher FD . . . . . . . . . . J02,SP18

Boyd DA $\ldots \ldots \ldots \ldots \ldots$ K01,K05,P03

Boyer N . . . . . . . . . . . . . . . P12

Brau N . . . . . . . . . . . . . . . . . . . P12

Bresee L . . . . . . . . . . . . . . . . . . SP07

Breznan D. ........................

Bronowicki J-P. . . . . . . . . . . . . P12

Brooks A .....................P10

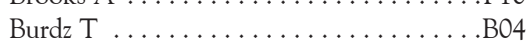

Burry $\mathrm{L} \ldots \ldots \ldots \ldots \ldots \ldots$. . . P09,SP09

Burstein B..................P32

\section{C}

Callery $S \ldots \ldots \ldots \ldots \ldots \ldots \ldots$ E01

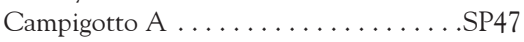

Casselman A . . ................... 30

Caswell D.....................P17

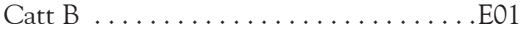

Chagla Z. ...................SP22

Chahil N .....................F01

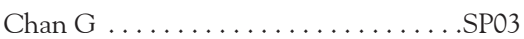

Chapman W .................... G05

Charest H ...................F03

Cheeptham N .................SP46

Chen J ...................... I05

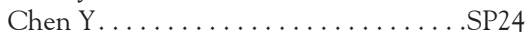

Chilvers M...................SP18

Chiu A . . . . . . . . . . . . . . . . . P09, PP09

Cho P........................E01

Choi K..........................204

Chokani K....................P18

Chong J . . . . . . . . . . . . . . . . . . . SP11

Chong M . . . . . . . . . . . . . . . . . D D 4

Chong $\mathrm{P} \ldots \ldots \ldots \ldots \ldots \ldots$. . . . . . . . .

Chong S .................... 105

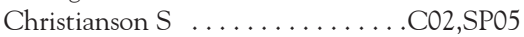

Chu K ....................F01

Chui L . . . . . . . A A03,I02,I05,SP42,SP43

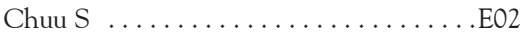

Clark S ......................... 04

Coburn B ................... C04

Cohen A ...................... J03

Cole J ....................... A04

Colemand B..................SP26

Compton G . ................SP10

Conly J . . . . . . . . . . . . . . . . . SP07,SP19

Connolly E. . . . . . . . . . . . . . . .SP34

Connors WJ . . . . . . . . . . . . . . . SP49

Constantinescu C . . . . . . . . . . . . . . J01

Conway B ................... J05

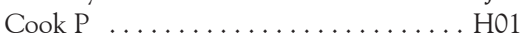

Cooke W ....................E02

Cooper JN . . . . . . . . . . . . . . . . . .P11

Corpus C . . . . . ................ E01

Craig J . ..........................

\section{$\mathrm{D}$}

D'Souza A ...................P06

Daigneault P................SP18

Daku D .................... A02

Daley P ............ A04,H02,SP10

Dalton B. ...................SP07

Davidson R .............. P23,SP40

Davies HD . . . . . . . . . . . . . . . . . E03

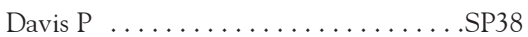

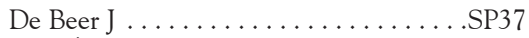

De L'Étoile-Morel S . . . . . . . . . . . . . . SP30

De Repentigny L . . . . . . . . . . . . . . . E03

De Serres G . . . . . . . . . . . . . . . J02

Deans I. . . . . . . . . . . . . . . . . SP31

Degagne P . . . . . . . . . . . . . . .SP12

Delannoy S ...................SP42

Delorme A ...................SP39

Delport J . . . . . . . . . . . . . . . . B03

Demczuk W . . . . . . . . . . . . . . D02

Denisuik A ............... L03,SP29

Deserres G . . . . . . . . . . . F03,SP18
Desjardins M ............... B04,K02

Dhanoa T.....................P15

Diaz Caballero J ................ C04

Diaz-Mitoma F. . . . . . . . . . . . . . . IA03

Dimitrova K ....................F04

Dionne M....................SP20

Dobson S . . . . . . . . . . . . . . . . . J02

Dolce P.........................A01

Donaldson S . . . . . . . . . . . . CO 4

Donkor K....................SP46

Donnenberg M. . . . . . . . . . . . . . .SP41

Dore GJ ....................P13

Doualla-Bell F . . . . . . . . . . . . . . . . I03

Doucette K....................SP17

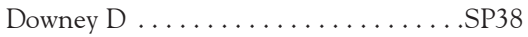

Downing M ................... G05

Doyle P......................SP34

Drebot M . ...................F04

Dresser L. . . . . . . . . . . . . . . . SP13

Drew T .................. B01,D03

Drews SJ ............F05,I01,SP16

Dubrovsky AS ................P32

Dunna S .......G01,G04,K01,K05,P03

Durand J....................SP48

Dushenski D. . . . . . . . . . . . . . . . .P25

Dust K . . . . . . . . . . . . . . . F05

Duvvuri VR ................FO2

\section{$\mathrm{E}$}

Edagiz S . . . . . . . . . . . . . . G02,K04

Eichhorst JC. ..................P17

Einarsson G . . . . . . . . . . . . . . .SP38

El Gamal A ......................P01

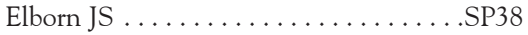

Elsayed S . . . . . . . . . . . . . . . . . . SP19

Emond $\mathrm{Y} \ldots \ldots \ldots \ldots \ldots \ldots \ldots . . . \ldots \ldots$

Ens C . . . . . . . . . . . . . . . . . . . CO2

Eshaghi A ................... F02

Everson GJ ..................P13

\section{F}

Fack P...................... 4 42

Faizullah $\mathrm{H} \ldots \ldots \ldots \ldots \ldots \ldots \ldots \ldots$ HO 4

Farrell DJ ........................ 01

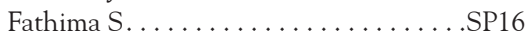

Fattouh R . . . . . . . . . . . . P16,SP06

Fernandes T . . . . . . . . . . . . . . . . P09, PP09

Fernando E. .................... .

Fernando K .................H06,P15

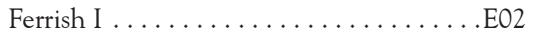

Finley R . . . . . . . . . . . . . . . . . .LO1

Forgie E .................. J04

Forgie S . . . . . . . . . . J04,SP32,SP48

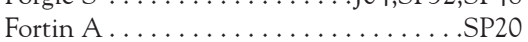

Fortin É ..............................

Fournier E .................... I03

Frame B .................. 21

Freilich BF ....................... 11

Frenette C . . . . . . . . . . . . . . SP45

Fuller J

L02,L05,P14

\section{G}

Gallant R...................P20

Gao Z . . . . . . . . . . . . . . . . . . SP05

Garber $\mathrm{G} \ldots \ldots \ldots \ldots \ldots \ldots \ldots$. . . . . . .

Garceau R . . . ............. A01,F05

Gariepy MC ................ J02

Gartner N. . . . . . . . . . . . . . . SP48 


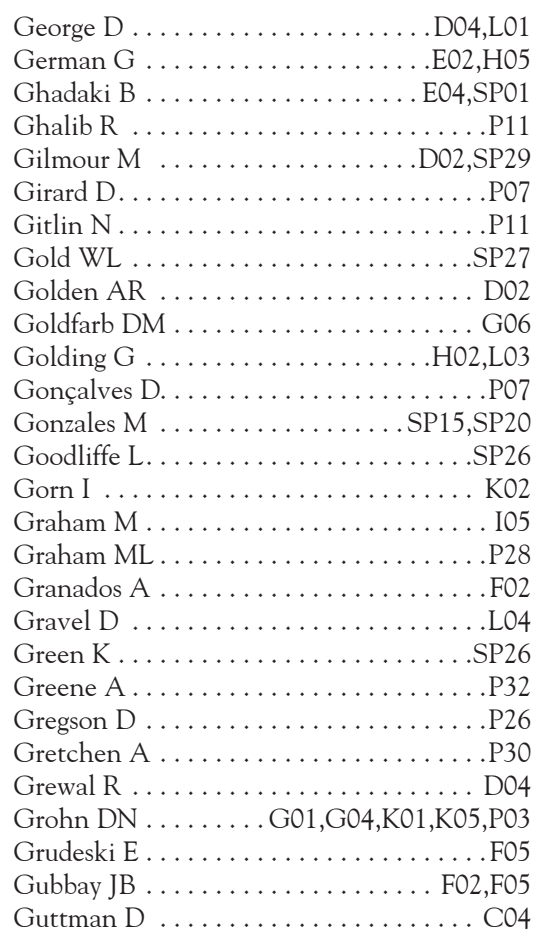

$\mathrm{H}$

Haider $\mathrm{S} \ldots \ldots \ldots \ldots \ldots \ldots \ldots . . \ldots$ SPO

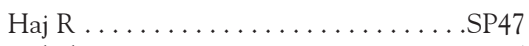

Hakobyan S . ................. J05

Haldane D . . . . . ........... I04,SP40

Halperin SA ................ J02

Hancock D .................... G05

Hao X-K ..................... E05

Harlan W......................P11

Harris D . . . . . . . . . . . . . . . SP37

Hatchette T ......F05,H01,P11,P20,SP40

Haworth-Brockman M. . . . . . . . . . P27

Hayden K.................... . 18

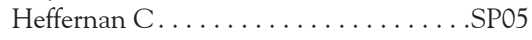

Heinstein C . . . . . . . . . . . . . . . .P20

Heitman S . . . . . . . . . . . . . . P26

Hennicken D . . . . . . . . . . . . . . P11

Henry B . ....................P15

Hézode C . . . . . . . . . . . . . . . . . P13

Hoang L . . . . ............ B04,CV04

Hoban DJ . . . ...... D02,L02,L03,L05,SP29

Hojgaard A. . . . . . . . . . . . . . . . P15

Holbein B..................P23

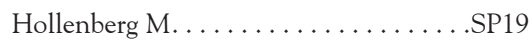

Holloway K . . . . . . . . . . . . . . . . F04

Holton DL . . . . . . . . . . . . . . SP04,SP49

Horsman GB ........ A02,B02,P17,P18

Hosseini B . . . . . . . . . . G02,K04

Hughes EA............. P11,P12,P13

Hughes $\mathrm{M} \ldots \ldots \ldots \ldots \ldots \ldots \ldots . . \ldots$. 04

Hui $\mathrm{C} \ldots \ldots \ldots \ldots \ldots \ldots \ldots \ldots \ldots \ldots \ldots \ldots \ldots \ldots \ldots$

Hwang D ..................... 04

I

Ilagan $\mathrm{R} \ldots \ldots \ldots \ldots \ldots \ldots \ldots \ldots$. B 1 Invik J . . . . . . . . . . . . . . SP16 Irfan N . . . . . . . . . . . E04,P10,SP22 Ishikawa $\mathrm{T} \ldots \ldots \ldots \ldots \ldots \ldots \ldots$. P26

\section{$\mathrm{J}$}

Jackson C...................P20

Jacobson IM . . . . . . . . . . . . . . P12

Jadavji T.

J01,SP07
Jamieson $\mathrm{FB} \ldots \ldots \ldots \ldots \ldots \ldots \ldots . \ldots \ldots$

Jansen B . . . . . . . . . . . . . . P14

Jayaratne $\mathrm{P} \ldots \ldots \ldots \ldots \ldots \ldots$ G03,K03

Jensen K......................P15

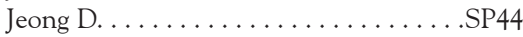

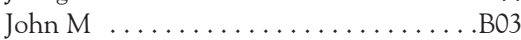

Johnston E . . . . . . . . . . . . . SP38

Johnstone J . . . . . . . . . . . .EO6

\section{K}

Kadkhoda K...................P30

Kahnamelli B.................P15

Kaita K......................P30

Karlowsky JA .... D02,G02,K04,L03,SP29

Karnauchow T .................F05

Katz K........................ .

Kelly M .................... 01

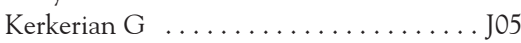

Kettner J.....................P27

Keynan Y . . . . . . . . . . . . . . . . SP12

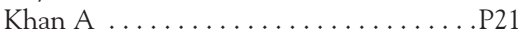

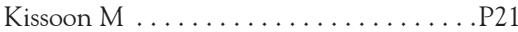

Kitzul R .........................

Knox N ..................... I05

Koeck E . . . . . . . . . . . . . . SP34,SP46

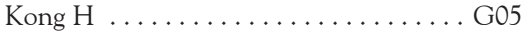

Kornherr P . . . . . . . . . . . . . . H04

Kotowich L . . . . . . . . . . . . . . SP24

Kovacs L . . . . . . . . . . . . . . . . E03

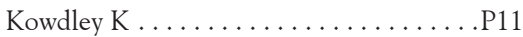

Krajden M ...................F05

Krajden S ..................... G05

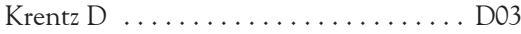

Kronfli N . . . . . . . . . . . . . . . . . . .SP01

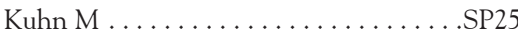

Kuhn S ................... J01

Kuppalli K ..................PO6

Kus JV ....................P16

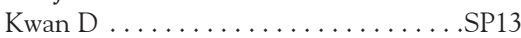

Kwo P....................... 13

\section{L}

Labrecque E . . . . . . . . . . . . . . . IA01

Laferriere C . . . . . . . . . . . . . SP11

Lagacé-Wiens P ........... B04,G02,K04 SP12,SP29

Lalezari J. . . . . . . . . . . . . . . . . P13

Lalezari JP. . . . . . . . . . . . . . . . . .P11

$\operatorname{Lam} \mathrm{C} \ldots \ldots \ldots \ldots \ldots \ldots \ldots \ldots . \ldots \ldots$

Lam F ..................... E06

Lam P................................ 26

Lands LC . . . . . . . . . . . . . F03,SP18

Langford B . . . . . . . . . . . . . . . . G05

Langley $\mathrm{J} \ldots \ldots \ldots \ldots \ldots \ldots \ldots \ldots$. E03

Langlois M-A......................

Lannigan $\mathrm{R} \ldots \ldots \ldots \ldots \ldots \ldots \ldots$. . . . . . . . .

Lapinsky S . . . . . . . . . . . . . . . . P25

Lata C. . . . . . . . . . . . . . . . . . SP04

Latonas $\mathrm{S} \ldots \ldots \ldots \ldots \ldots \ldots \ldots \ldots$. . . . . . . . . . .

Lavigne C....................PO7

Lawandi A .........................................

Lawitz E . . . . . . . . . . . . . . . . . P11

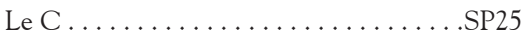

Le Saux N .................... E03

LeBlanc J . . . . . . . . . . . . . . F F05,P20

Lechiile $\mathrm{K} \ldots \ldots \ldots \ldots \ldots \ldots \ldots$. G06

Leduc $S \ldots \ldots \ldots \ldots \ldots \ldots \ldots \ldots$. . . . . . . . . .

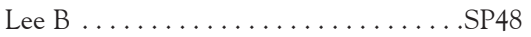

Lee M-K .................. 6 P15

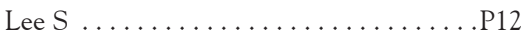

Lee TC .................SP30,SP45
Lee $\mathrm{W} \ldots \ldots \ldots \ldots \ldots \ldots \ldots \ldots \ldots \ldots$

Leis JA ................. E01,SP27

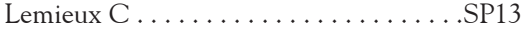

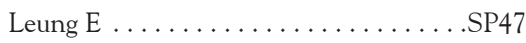

Leung $\mathrm{O} \ldots \ldots . \mathrm{G} 01, \mathrm{G} 04, \mathrm{~K} 01, \mathrm{~K} 05, \mathrm{P} 03$

Lévesque $\mathrm{S} \ldots \ldots \ldots \ldots \ldots \ldots \ldots$. . . . . . . . . . .

Levett PN .......A02,B02, F05,P17,P18

Levin J . . . . . . . . . . . . . . . . P12

Li A ....................... 22

Li V .........................

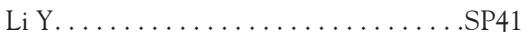

Lindsay R ................F04,H01

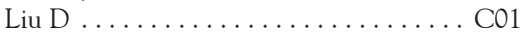

Lloyd $\mathrm{V} \ldots \ldots \ldots \ldots \ldots \ldots \ldots \ldots . . \ldots \ldots$

Lo P .........G01,G04,K01,K05,P03

Loeb M .................E04,G06

Long D . . . . . . . . . . . . . . . . . . .SP05

Long R . . . . . . . . . . . . . . . . . SP05

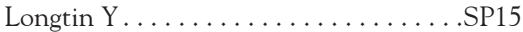

Lother S . . . . . . . . . . . . . . . .SP12

Lou A ......................... 20

Low $\mathrm{DE} \ldots \ldots \ldots \ldots \ldots \ldots \ldots \ldots \ldots \ldots \ldots \ldots \ldots \ldots \ldots$

Luinstra $\mathrm{K} \ldots \ldots \ldots \ldots \ldots \ldots \ldots \ldots \ldots$ IAO 2

\section{M}

Ma $\mathrm{A} \ldots \ldots \ldots \ldots \ldots \ldots \ldots$ I02,SP43

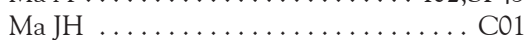

MacDonald J ................ J01,P20

MacFadden D .................. J03

MacFadden DR . . . . . . . . . . . . . SP27

MacLaggan T ..................SP25

MacLean G . .................... H01

Mah A ................................

Mahony J ....................... I05

Main C.................. P08,P19

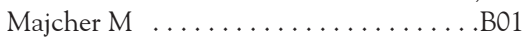

Mak A . ..................... F01

Mak S........................ 15

Makowski K ...................F04

Maldarelli G. ..................SP41

Malejczyk K................... A02

Mallet M .................. A01

Man S .................................. 15

Mandelboim M ...............SP36

Maregmen J ...................P22

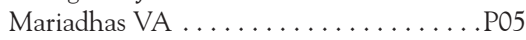

Marr P ........................

Marra F .................. D04

Martin I . . . . . . . . . . . D02,I04,SP12

Martineau C . . .................F05

Martinez De La Pena C.............SP41

Masud S . . . . . . . . . . . . . . . . . SP33

Matukas LM..................SP47

Maze Dit Mieusement L . . . . . . . . . . J J03

Mazhani L . . . . . . . . . . . . . . . . . G22, G06

Mazzulli T. . . . . . . P22,F05,G01,G04,K01

................. K05,P03,P16

McCaffrey W . . . . . . . . . . . . . P08

McCarten J. . . . . . . . . . . . . . . . . . SP28

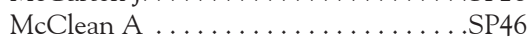

McClure J....................SP19

McConnell M . . . . . . . . . . . . . P08

McCorrister S . ....................

McCready W . . . . . . ................ D01

McCreight $\mathrm{L} \ldots \ldots \ldots \ldots \ldots \ldots \ldots \ldots . \ldots \ldots$

McDonald C . . . . . . . . . . . . . . . P25

McDonald EG ................SP30

McDonald RR ............... A02,F05

McDougall C . . ................... E06

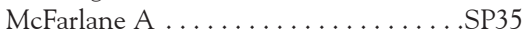

McGeer AJ.............J03,SP06,SP26 
McGrath S. . . . . . . . . . . . . . SP38

McIlreavey L . . . . . . . . . . . . . . SP38

McKim A......................

McNabb A ..................... F05

McNeil S . . .......................

McPhee F.......................P11

Meier-Stephenson V . . . . . . . . . . . . P26

Melano RG . . . . . . . . . . . . . . . . . SPO6

Mendelson E . . . . . .............SP36

Mertz D . . . . . . . . . . P P10,SP31,SP37

Miller M..................SP21

Mina N . . ............ A02,B02,P17

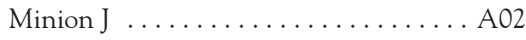

Mitchell R ....................204

Mithoowani S ..................P10

Mokomane M ..................G06

Mollison L . . . . . . . . . . . . . . . . . . . P12

Monkman L . . . .............. H03

Moorad JB ................... G06

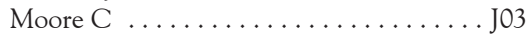

Moore R .................... H05

Morgan M .................... 25

Morris A. .............P09,P25,P25,SP09

Morshed M ............ F01,H06,P15

Moses J . . . ..................... B01

Mothana R.......................P01

Mubareka S ............... F05,SP24

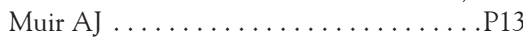

Muller M . .................SP47

Mulvey MR . . . . . . L L01,K01,SP12,SP29

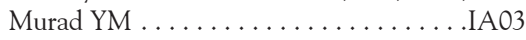

Murray J ....................SP25

\section{$\mathrm{N}$}

Nadarajah J . ................SP47

Nadon C . . . . . . . . . . . . . . . B01,SP42

Naidu P . . . . . . . . . . . . . . . . .SP46

Nassar B . . . . . . . . . . . . . . . . . P20

Needle R . . . . . . . . . . . . . . A04,F05

Nelson DR . . . . . . . . . . . . . . . . . P11

Neudorf N . . . . . . . . . . . . . . . A02

Ng K........................

$\mathrm{Ng} \mathrm{M} \ldots \ldots \ldots \ldots \ldots \ldots \ldots \ldots . \ldots \ldots$

Nichol KA ................... D02

Nicolle L . . . . . . . . . . . . . . . . SP10

Nix E .................... D01

Nokhbeh R ...................IA03

\section{$\mathrm{O}$}

O'Brien $\mathrm{K} \ldots \ldots \ldots \ldots \ldots \ldots \ldots$. E03

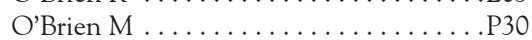

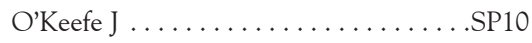

O'Neill C.......................SP31

O'Shaughnessy A ............... J03

Obarianyk A .................... 02

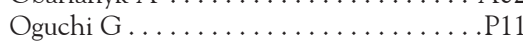

Oommen T ..................SP28

Ortiz-Lasanta G..................P11

\section{$\mathbf{P}$}

Pabbaraju K . . . . . . . . . . . . . . I01

Pagotto F ...................... I05

Papenburg J . . . . . . . . . . . . F03,SP20

Papoushek C . . . . . . . . . . . . . . .SP13

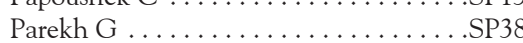

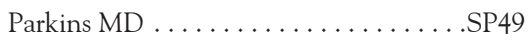

Parmeswaran H . . . . . . . . . . . . . . . P06

Parquet M....................P23

Partovi M ....................... H03

Patel SN . . . . . . . . . . . . . . . . . . SP06

Patrick D . . . . . . . . . . . D04,L01,P15

Patriquin $\mathrm{G} \ldots \ldots \ldots \ldots \ldots \ldots \ldots . . \ldots \ldots 02$
Patterson-Fortin LM . . . . . . . . . . I05,SP43

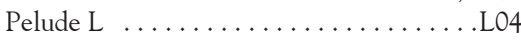

Penney C . . . . . . . . . . . . A04,H02,SP10

Perez J. . . . . . . . . . . . . . . . . . IA03

Pernica JM $\ldots \ldots \ldots \ldots \ldots \ldots$ G06,J02

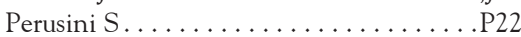

Peterson T . . . . . . . . . . . . . . . . SP42

Petrescu D . . . . . . . . . . . . . . .SP27

Petri B . . . . . . . . . . . . . . . . . SP19

Petrich A . . . . . . . . . . . . . . . . . F05

Petruccelli D . . . . . . . . . . . . . SP37

Pham-Huy A . . . . . . . . . . . . . . . J02

Pickles E .................... H04

Pockros PJ ............... P11,P13

Poliquin G . . . . . . . . . . . . . G02,K04

Pomeranz G . . . . . . . . . . . . . . . . . . SP36

Poordad F.................. P12,P13

Popiel K....................SP21

Porter V . . . . . . . . . . . . . . . . . . .SP24

Poutanen SM ....... G01,G04,K01,K05

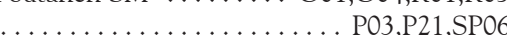

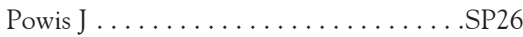

Prematunge $\mathrm{C} \ldots \ldots \ldots \ldots \ldots \ldots \ldots$. . . . . . .

Priyantha R . . . . . . . . . . . . . . . . SP08

Prystajecky N ................ I05

Purych D .........................

Putz J

.P17

\section{Q}

Quach C .......... F03,J02,P32,SP11

\section{$\mathrm{R}$}

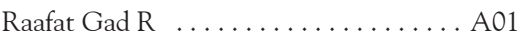

Rabinovitz M....................P11

Radko S . . . . . . . . . . . . . . . . F06

Rahman P .......G01,G04,K01,K05,P03

Rajapakse N.................SPO7

Ramdass T . . . . . . . . . . . . . . . . P21

Ramji A .........................

Rana K.....................P11

Ravendhran N . . . . . . . . . . . . . . . .P11

Raybardhan S. . . . . . . . . . . P09,SP09

Read T ...........................

Rehan M ......................P19

Reimer A . . . . . . ............... I03

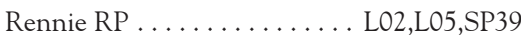

Reyes R . . . . . . . . . . . . . . .....LO1

Ricci G ................ K01,K05,P03

Ricciuto D . . . . . . . . . . . P09,SP09,SP28

Richardson S . . . . . . . . . . E03,F05,P31

Roberts C..................P2O

Robertson J . . . . . . . . . E06,E03,SP32

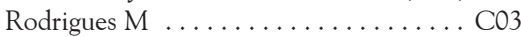

Roscoe D . . . . . . . . . . . . . . SP33,SP34

Rosenfeld P . . . . . . . . . . . . . . . . . FO2

Rostom M ....................SP13

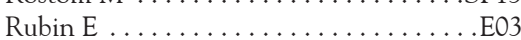

Rubin J . . . . . . . . . . . . . . . . . . SP08

Rutherford C . . . . . . . F05,G03,H03,IA02

Ryan L . . . .................SP28

\section{$\mathrm{S}$}

Saini $\mathrm{V} \ldots \ldots \ldots \ldots \ldots \ldots \ldots . \ldots . \ldots . \ldots . \ldots 1$

Saltzman I . . . . . . . . . . . . . . SP27

Sauve R .....................E03

Savage K.......................P23

Saxinger L . . . . . . . . . . . . . . . SP35

Scheifele D ....................F03

Scheifele G. . . . . . . . . . . . . . . .SP18

Schleihauf E .................... I04

Schreiber Y. . . . . . . . . . . . . .SP44
Science M .....................SP09

Scott AN . . . . . . . . . . . . . . . . . . SP16

Sepe T ........................

Seyed Salehi M ................P21

Seyer K . . . . . . . . . . . . . . . . . . I05

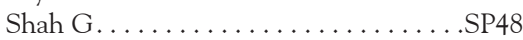

Sharma M ................. 02, SP05

Sharma S ................... J05

Sheikh AM ..................... 11

Shen A........................ . . .

Shen YH ...................... E03

Shephard A . . . . . . . . . . . . . . . P02

Shokoples S . . . . . . . . . . L02,L05,P14

Showler A . . . . . . . . . . . . . . . P09,SP09

Shuel M . . . . . . . . . . . . . . D03,P18

Shumilak G . . . . . . . . . . . . . . .SP23

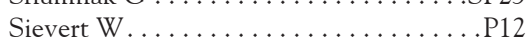

Simmonds K. . . . . . . . . . . . . . . . .SP16

Simner P . . . . . . . . . . . . . . . . . SP29

Simor A ................E01,SP24

Simpson K ...................E03

Simpson Y . . . . . . . . . . . . . . . F01

Skorchid D. . . . . . . . . . . . . . . . P08

Slade $\mathrm{P} \ldots \ldots \ldots \ldots \ldots \ldots \ldots \ldots \ldots$

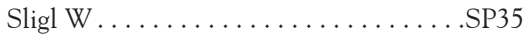

Smart G . . . . . ........................

Smieja M . . . . . . . . . . F05,G06,IA02

Smith S . . . . . . . . . . . . SP17,SP35

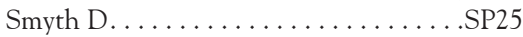

So J ...................... J03

Sohrabi M ................... HO4

Somayaji R. . . . . . . . . . . . . . . . . . SP04

Spreitzer D ................... L03

Stals S ........................ 07

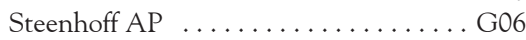

Stefanovic A . . . . . . . . . . . . . . . CV04

Steinberg M . . . . . . . . . . . . . . . P09, SP09

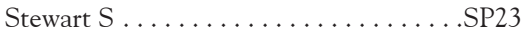

Stordal K ..................... G06

Strikwerda A .................... B03

Strong D. . . . . . . . . . . . . . . J01

Stuart JI . . . . . . . . . . . . . . . F06

Suresh S . . . . . . . . . . . . . . . . SP32

Swenson ES . . . . . . . . . . . . P12,P13

Synnes A . . . . .................E03

\section{$\mathrm{T}$}

Taggart LR . . . . . . . . . . . . . .SP47

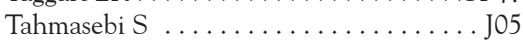

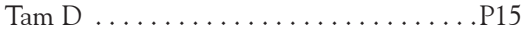

Tambalo D . . . . . . . . . . . . . . . . .SP19

Tan B . . . . . . . . . . . . . . . . E03

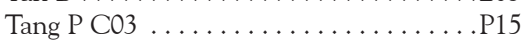

Tellier R ................F05,I01

Thirion DG . . . . . . . . . . . . . . . SP20

Thomas E .................. J03

Thuluvath P...................... 11

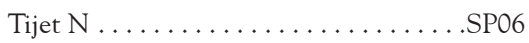

Tiller S ..................... A04

Tipples G . . . . . . . . . . . . . . . . F05,I01

Tomlinson G . . . . . . . . . . . . . P09,SP09

Top KA ................... J02

Tossonian H ................. J05

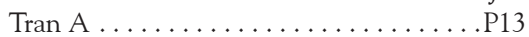

Tran D . . . . . . . . . . . . . . J02

Tremblay C ................... I03

Trimi X .........G01,G04,K01,K05,P03

Trout-Yakel K ..................... B01

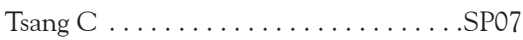

Tsang RSW . . . . . . . . . D01,D03,P18

Tschetter L . . . . . . . . . . . . . . . .SP42

Tullis DE .................. C04 


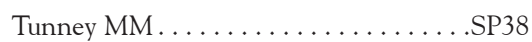

Turenne CY . . . . . . . . . . . . . . . B02

Turnbull L . . . . . . . . . . . . . L02,L05

Tyndall M.......................... 44

\section{$\mathrm{U}$}

Ulanova M D01

\section{V}

Vallieres E ................SP38

Van Den Broek K. . . . . . . . . . . . . . P25

Van Domselaar G . ................ I05

Vanderkooi O................SP07

Varunok P .....................................

Vaudry W .................... J02

Vaughan $S \ldots \ldots \ldots \ldots \ldots \ldots \ldots$. . . . . . . .

Vayalumkal JV . . . . . . . . . . . . SP03,SP07

Vearncombe M ....................E01

Vomiero G.....................

\section{W}

Wagner K......................SP46

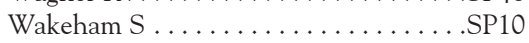

Wang P ...................... 04

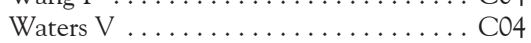

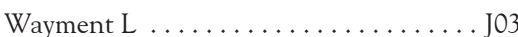

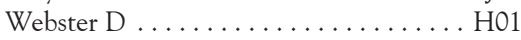

Wells K ........................ A04

West S ........................... 25

Westmacott G ................... B01

Whellams D ....................SP22

Whitney H ................ H02

Whyte K. . . . . . . . . . . . . . . D03,P18

Wiebe D ............................

Wilcox T $\ldots \ldots \ldots \ldots \ldots \ldots \ldots$

Willcocks D ................. J03

Willey BM . .G01,G04,K01,K05,P03,P16,P21

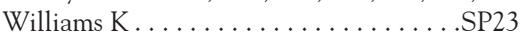

Winemaker M ..................SP37

Wolfe J . . . . . . . . . . . . . . . C02,SP05

Wong B . . . . . . . . . . . . . . . . . .EO1

Wong F $\ldots \ldots \ldots \ldots \ldots \ldots \ldots \ldots \ldots \ldots$ E01

Wong J .................... J03

Wong Q . . . . . . . . . . . . . F01,H06,P15

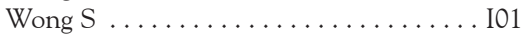

Wong T ................ SP34,CV04

Wood M .................... J04

Woods $\mathrm{O} \ldots \ldots \ldots \ldots \ldots \ldots \ldots \ldots . . . \ldots 22$

Woolston K ...................SP38

Wu K . . . . . . . . . . . . . . . .SP19

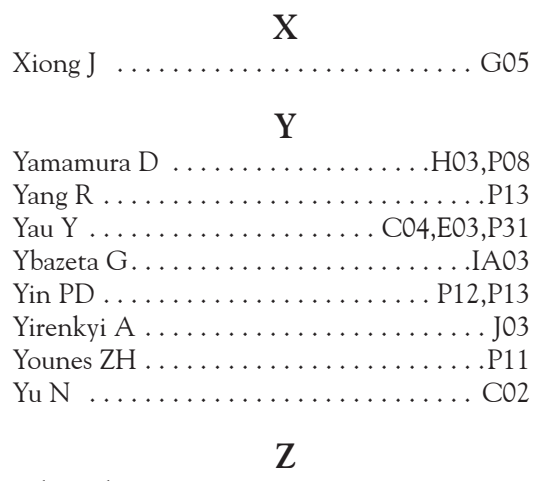

Zahariadis $\mathrm{G} \ldots \ldots \ldots \ldots \ldots \ldots \ldots \ldots$ F06

Zelyas N....................SP17

Zhanel GG .......D02,L02,L03,L05,SP29

Zhang J . . . . . . . . . . . . . . . I05,SP19

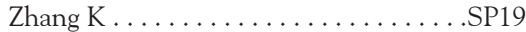

Zhang L . . . . . . . . . . . . . . . . . . . . . . J J03

Zhang Y ...................... C04

Zhou HY ..................... P14,SP0

Zybeshari S...................P02 


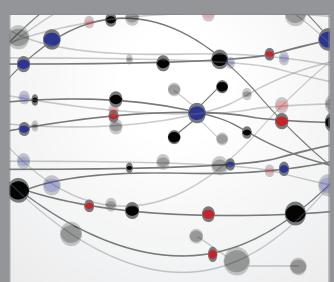

The Scientific World Journal
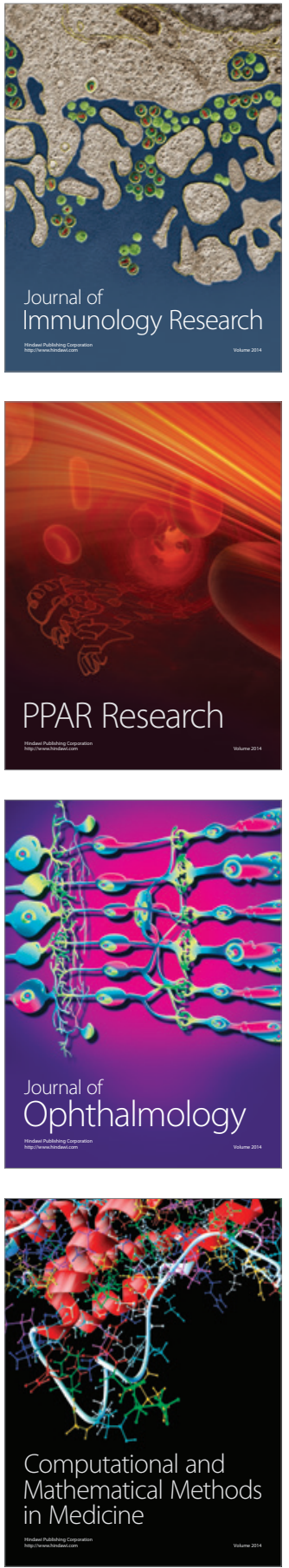

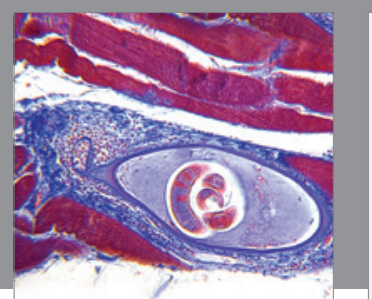

Gastroenterology Research and Practice

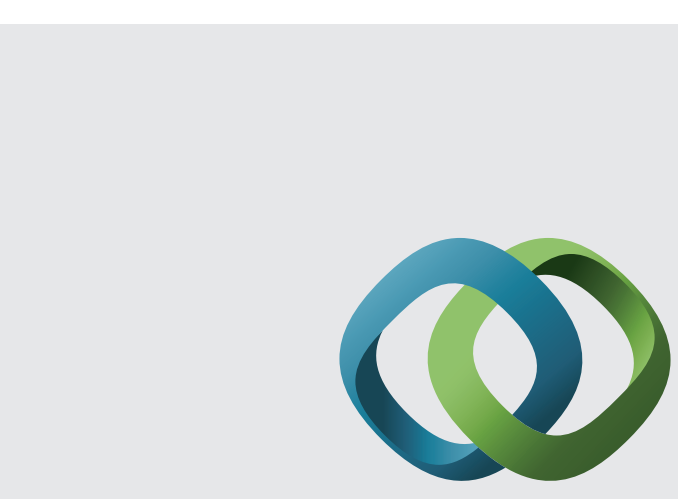

\section{Hindawi}

Submit your manuscripts at

http://www.hindawi.com
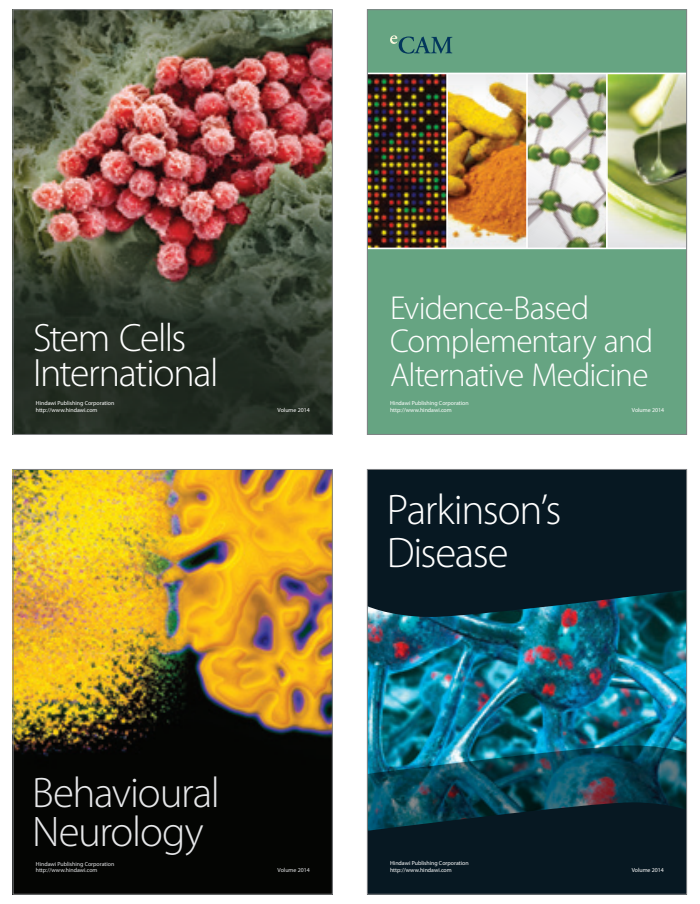
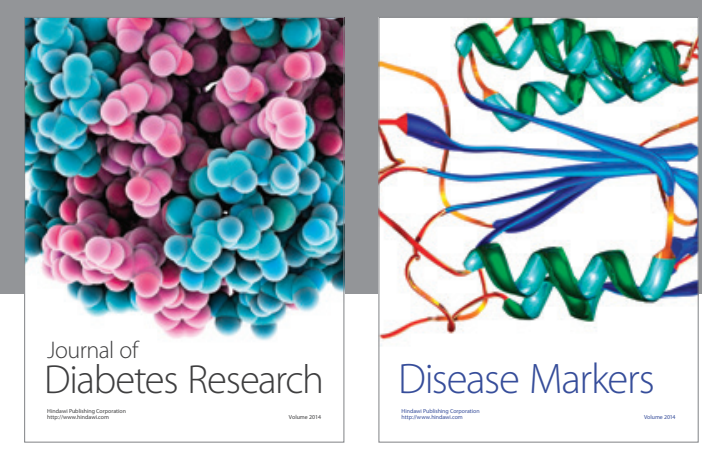

Disease Markers
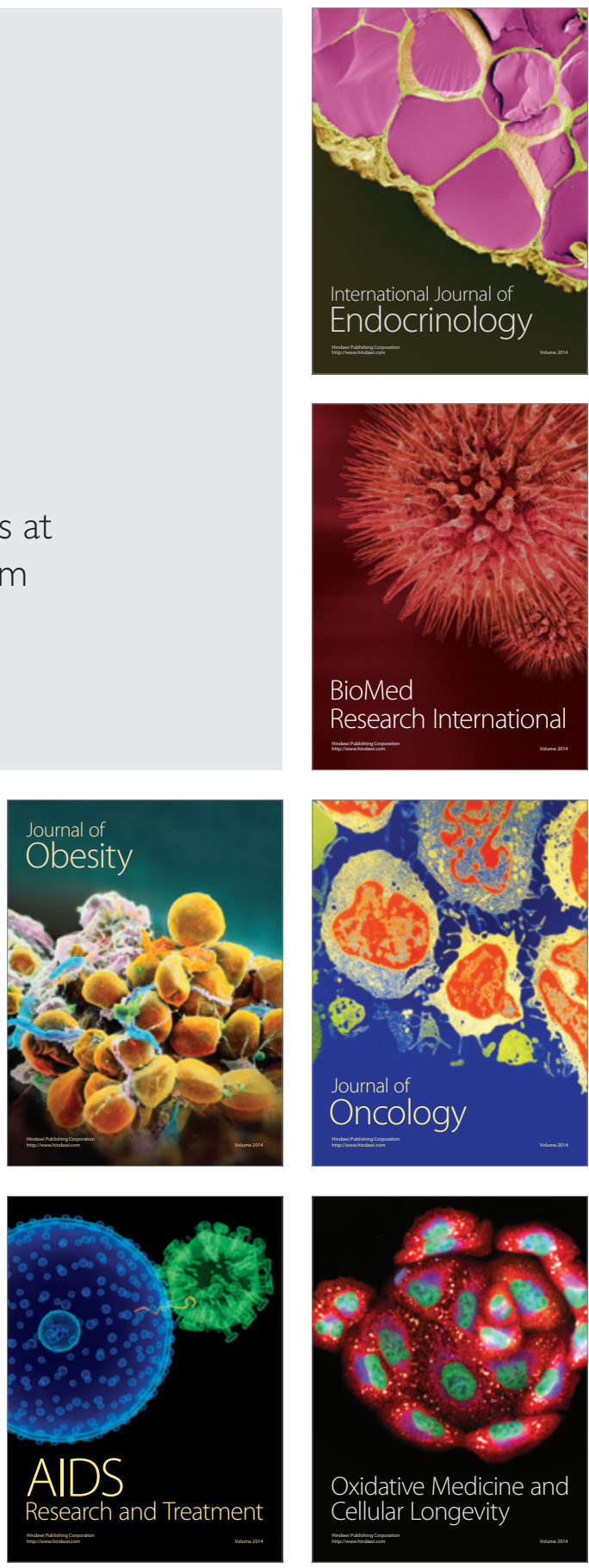